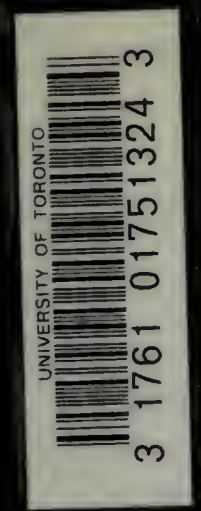





Digitized by the Internet Archive in 2007 with funding from Microsoft Corporation 
$2 / 91-3$
SYNCHRONOUS MOTORS

$2191-3$
SYNCHRONOUS MOTORS

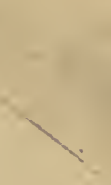




\section{McGraw-Hill BookCompany}

\section{Publishers of Books for}

Electrical World The Engineering and Mining Journal

Engineering Record

Railway Age Gazette

Siǵnal Engineer

Electric Railway Journal

Engineering News

American Machinist

American Engineer

Metallurgical and Chemical Engineering

Coal Age

Power 


\title{
SYNCHRONOUS MOTORS AND CONVERTERS
}

\author{
THEORY AND METHODS \\ OF CALCULATION AND TESTING
}

BY

ANDRÉ E. BLONDEL

Graduate and Professor, National School of Bridges and Highways of France; Member of the Legion of Honor of France; Honorary Member of the American Institute of Electrical Engineers; Member of Numerous Scientific, Technical, and Engineering Societies in France and other European Countries

TRANSLATED FROM THE FRENCH

BY

C. O. MAIlloUX, M.E., M.S.

Consulting Electrical Engineer

WITH ADDITIONAL CHAPTERS

BY

COMFORT A. ADAMS, S.B., E.E.

Professor of Electrical Engineering in Harvard University

M GRAW-HILL BOOK COMPANY

239 WEST 39TH STREET, NEW YORK

6 BOUVERIE STREET, LONDON, E.C. 
COPYRIGHT, 19I3,

BY THE

MCGRAW-HILL BOOK COMPANY

$\checkmark B R A R$

NกY I 1967

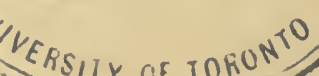




\section{TRANSLATOR'S PREFACE}

Prof. A. Blondel was the first writer to publish a systematic, comprehensive work on Synchronous Motors; and his book, although it has now been before the public several years, still remains the leading work on that important subject. Owing to the non-existence of an English edition, it is not, however, as well known and as much appreciated as it deserves to be, by English-speaking readers.

The translation of this celebrated, one might almost say classical, work into English, was undertaken at the suggestion of teachers and others who were desirious of making more extensive use of the work than is possible if the French text alone is available.

The author and the publisher both accepted the suggestion that the scope and the usefulness of the book might be increased materially by including in it some reference to "Rotary Converters." Excellent material for this purpose was already available in the form of a paper presented by Prof. Blondel, at the Electrical Congress in Paris, in 1900. Two other papers presented by him at the Electrical Congress at St. Louis, in I9O4, also were of sufficient interest in this connection to make their reproduction desirable.

It was decided to separate the contents of the book into three distinct parts. Part I, relating to Synchronous Motors, corresponds to the original French work on Synchronous Motors. The author himself corrected the French text, and he also supplemented it with much new matter while the translation was in process. The proofs of the English text were submitted to several persons who were well qualified to criticise the text and suggest improvements therein. The French text of Part I is also supplemented by an additional chapter contributed by Prof. C. A. Adams, of Harvard University. Part I may therefore be considered fairly well brought up to date. Part II, relating to Synchronous or Rotary Converters, is made up of old and new matter. The old matter (Chapters I and II), constitutes a translation of Prof. Blondel's Paris-Congress 
paper of I900. The new matter consists of three chapters by Prof. Blondel, and a chapter contributed by Prof. C. A. Adams. Part III contains the reprints of the two papers presented by Prof. Blondel at the St. Louis Electrical Congress in I904, relating to the application of his "two-reaction" method to alternators.

A very few modifications and additions have been made in the text by the translator. His own strong objections to the terms "wattless" and "watted" made him very willing to eliminate them; and the recent action of the Standards Committee of the American Institute of Electrical Engineers in sanctioning and recommending "reactive" and "active" as substitutes, furnished the incentive and the pretext for doing this, even after a considerable portion of the type had been set. This book will, therefore, be the first book in which "wattless" and "watted" are replaced by "reactive " and "active" respectively.

The translator desires to express his gratitude and sincere thanks to all who have encouraged and assisted him in the preparation of this book for the press. Special acknowledgment should be made of the very valuable services rendered by Prof. C. A. Adams. He was the first to suggest the publication of this book, and he has made many excellent suggestions, besides contributing two new chapters, and also reading and correcting the proofs of the entire book. Special thanks are also due to Prof. E. J. Berg, of the University of Illinois, for his very thorough reading of the proofs of Part I, and for very excellent suggestions for changes and additions made by him, some of which are incorported in notes inserted in the text, and identified by the initials "E.J.B." Thanks for reading the proofs of Part I, and making corrections or suggestions are due to the following:

Prof. D. C. Jackson, of Massachusetts Institute of Technology; Profs. W. I. Slichter and Morton Arendt, of Columbia University; Prof. H. H. Norris, of Cornell University; Mr. A. L. Jones, of the General Electrical Company.

Thanks are due to Mr. C. W. Stone, of the General Electric Company, and Mr. W. S. Rugg, of the Westinghouse Electric and Manufacturing Co., for the data relating to American Synchronous Motors, given in Appendix B; and also to the Edison Illuminating Company of Detroit, Mich., and to Mr. A. A. Meyer of its engineering department, for the information relative to the practical use of synchronous condensers contained in Appendix C. 
The Translator desires to make special acknowledgment of the great courtesy and kindness of the distinguished author, Prof. André Blondel himself. The preparation of this book for the press has been a labor of love which has occupied very pleasantly and profitably a portion of the leisure moments of the translator. $\mathrm{He}$ considers himself remunerated amply, for the work involved, by the great privilege which has been one of the perquisites incidental. to the task, namely, that of closer personal contact and acquaintance with the author; and he is very glad to have had the opportunity, through this translation, to help make the work and the talents of the author better known, as they deserve to be; for, unquestionably, Prof. Blondel is one of the great productive workers of our time in pure and applied electrical science. His work, great as it is in itself, becomes really wonderful and phenomenal, when the circumstances under which it has been done are realized and appeciated: Though handicapped most unfortunately, by protracted serious ill health and physical suffering, he has, nevertheless, kept well in the front rank with his more fortunate contemporaries and colleagues in the entire world; and he has achieved fame and renown by great mental powers, by wonderful originality and versatility, not only as a scientist, a teacher, and an author, but also as an inventor, an engineer, and an expert. The great respect which is inspired by the prodigious quantity and the superior quality of Prof. Blondel's work turn to absolute wonder and to profound admiration, before the wonderful activity and the untiring energy of his highly gifted, well-trained mind. No tribute of praise is too great for the work of this man, who is at the same time a genius and a hero, with an innate love of science and a devotion to scientific progress which uphold and uplift him, and urge him onward, quand même, in spite of ill-health and physical suffering, to new researches and new achievements.

NEW YoRk, December, rgr2.

The Translator. 



\section{CONTENTS}

PAGE

TRANSLATOR'S PREFACE......................... v

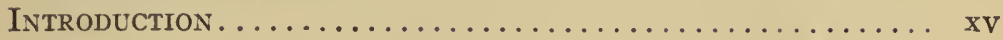

\section{PART I}

Chapter I.-General Principles of Synchronous Motors. . .

Construction - Experimental Properties - Case of Equal Electromotive Forces-Case of Unequal Electromotive Forces-Elementary Explanation of Polyphase Synchronous Motors-Elementary Explanation of Single-Phase Synchronous Motors-Equations of Synchronous Motors, Analytical Theory-Cașe of Symmetrical Polyphase MotorsGraphical Representation of Operative Conditions, Blakesley's MethodEquation of the Synchronous Motor by the Method of Complex Variables-Excitation of Synchronous Motors.

\section{Chapter II.-Detailed Study of Operation with Normal}

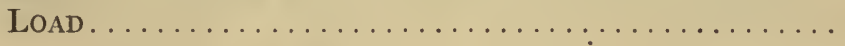

I. PRINCIPLES OF THE ELEMENTARY THEORY - NOTATION PRINCIPLE OF BIPOLAR DIAGRAMS

Bipolar Diagram of the First Kind-Motor-Vector $E_{2}$ taken as Fixed Axis-Applications of the Diagram of the First Kind-Line of Equal Power Occurring with Constant Excitation-Lines of Equal PhaseLimit-Circle of Current-Algebraical Relations Deduced from the Diagram-Numerical Example-Diagram of the Second Kind. The Vector of the Generator E.M.F., $E_{1}$, as a Fixed Axis-Power-Values as Function of the Lag-Angle 0-Use of this Diagram for the Study of Different Loads-Curves of Constant Electric Power of the Motor when the Generator has Constant Excitation-Current-Limit CircleLines of Equal Phase-Numerical Example. 
II. OPERATION OF A MOTOR WITH CONSTANT EXCITATION, SUP-

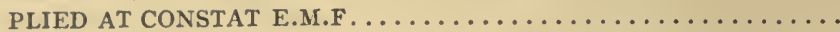

Maximum Power-Means of Determining the Practical Stability of Synchronous Motors-Variations of Stability with Operating ConditionsNumerical Example.

III. COMPARISON OF POSSIBLE OUTPUTS AT CONSTANT LOAD WITH VARIOUS EXCITATIONS. CONSTANT POTENTIAL SUPPLY....

Existence of a Current-Minimum-V-Curves-Use of Diagram of the First Kind-Predetermination of V-Curves-Theoretical Form of V-Curves-Curve of Reactive Current-Expression for Reactive Current - Comparison of Outputs which the Same Alternator Can Develop with the Same Terminal Voltage, when used either as a Generator or as a Motor.

IV. INFLUENCE OF MOTORS ON THE GENERAL OPERATION OF AN A.C. ELECTRICAL DISTRIBUTION SYSTEM............

Effect of Current of Synchronous Motors on Distribution SystemsCompensation with Respect to the Line or Circuit-First Numerical Example-Second Numerical Example-Economic Study of Compensation for the Line of Means of Motors Running without Lgad-Saving in Cost of Equipment-Saving in Annual Operating Cost-Numerical Example-Table I, Saving in Cost of Equipment-Table II, Saving in Operating Cost-Table III, Numerical Example-Economy of Compensation for the Distributing System by Means of Synchronous Motors Running with Load-Numerical Example-Regulation of DistributionVoltage-Compensation with Respect to the Generators-Numerical Example-Comparison between Synchronous and Induction MotorsUse of Synchronous Motors to Raise Power-factor in America.

\section{Chipter III.-Additions to the Theory. Second Approx-}

IMATION . . . . . . . . . . . . . . . . . .

Imperfections of the Theory-Variations of Reactance with Lag of Current and Saturation of Fields. Armature Reaction-First Application of Corrected Diagram-Determination of Reactive Current as a Function of the Excitation, with Constant Active Current-Particular Case where the Permeability of the Field-Circuit is Constant and the Two Reaction-Coefficients are Equal-Second Application. Operation with Constant Excitation, on Constant-Potential Supply SystemV-Curves-Influence of Field-Saturation on Stability-Influence of the Wave-Form of E.M.F.-Simplified Diagrams. 
Chapter IV.-Operation of Synchronous Motors. HuntING ......................... ro6

Starting by Direct Current-Starting with Alternating Current by Polyphase Motors-Synchronism-Observations on the E.M.F. Induced in the Poles-Accessory Starting Apparatus. Installation of Synchronous Motors-Starting of Single-Phase Machines-Theory of Initial Synchronizing-Separate Excitation-Field Due to a Commutated Current-Oscillations of Synchronous Motors-Short-Period OscillationsDamping of Oscillations-Long-Period Oscillations.

Chapter V.-Tests of Synchronous Motors

Characteristic Curves-Measurement of Efficiency-Experimental Tests-Advantages and Disadvantages of Synchronous Motors.

Chapter VI.-Other Motors Operating Synchronously without Direct-Current Excitation............. I4I

Reaction Synchronous Motors-Synchronous Motor with Alternating Fields.

Chapter VII.-Bipolar Diagram of the Second Kind in Ampere-Turns........................ 147

Introduction-Diagram Transformations E.M.F. Diagram-Approximate Diagram-Extreme Cases-Mechanical Analogue-Length of Air-Gap.

Chapter VIII.-Gentralization of Diagram for Coupled Synchronous Machines..................... I66

\section{PART II}

Chapter I.-General Diagrams Deduced from the Diagram FOR Synchronous Motors .................. I72

Introduction-Notation-Generalities. Reduction of all Armature Reactions to the Single Direct Reaction-Factors Determining the Practical Conditions of Operators. 
I. CONDITIONS OF ELECTRIC-CURRENT SUPPLY TO ROTARY CONVERTERS . . . . . . . . . . . . . . . . . . .

Fundamental Diagram-Fundamental Equation-Application of the Diagram. Representation of Converter Operation with Constant Potential at Primary Terminals and at Brushes-General Case. Reactive Current Values for a Given Voltage Variation as a Function of the Load-Most Suitable Value of Current-Supply Voltage-Most Suitable Value of Reactance-Regulation of Voltage at Terminals by Variation of the Supply E.M.F.-Regulation of Voltage at Terminals by Variation of Reactance-Power-Factor of the Generator.

Chapter II.-Predetermination of the Field-Excitation OF ROTARY CONVERTERS . . . . . . . . . . . .

Characteristic Features of the Rotary Converter-CompoundExcitation. Different Factors of this Excitation-Determination of Reactive Current as a Function of the Excitation when the Active Current is Constant, then when the Power is Constant, the Generator E.M.F. being always Constant-Different Values of the Excitation, with Constant Power and Constant Potential. V-Curves for Constant PotentialUpper Limit of Reactive Current-Lag-Characteristics of Rotary Converters at Constant Potential-Effective Characteristic of Rotary Converter under Ioad-Application in the Case of Separate ExcitationApplication to the General Case of Self-Excitation-Regulation of Supply E.M.F. by Compounding of the Generator-Regulation of Voltage by Varying the Reactance $X$ in the Circuit-Possibility of Suppressing the Shunt-IVinding-Conclusion.

Cilapter III.-Stability of Operation of Rotary Con-

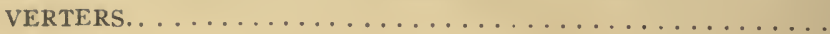

Chapter IV.-Operation of Severai. Rotary Converters in Parallei. . . . . . . . . . . . . . . .

Inherent Oscillations or Pumping of Converters Connected in ParallelUse of Rotary Converters for Transforming Direct into Alternating Current-Other Special Applications of Converters-Phase-Converters.

Chapter V.-Voltage Ratio in Syncironous Converters with Special Reference to the Split-pole Con-

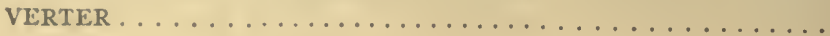




\section{PART III}

Chapter I.-Methods of Calculation of the Armature Reactions (Direct and Transverse) of Alter-

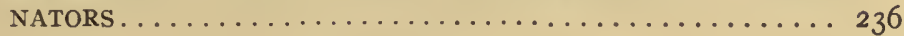

Principles of the Theory of Two Reactions-Diagram of E.M.F.'s and Current of an Alternator with Unsaturated Armature and with Saturated Field Magnet-Diagram of Ampere-Turns in the Case of Unsaturated Armature-Remark No. I, Upon the Case of an Unsaturated Armature-Remark No. 2, Upon the Subject of Diagram No. I-The Case of a Saturated Armature-Local Corrections of the Air-Gap Due to Saturation (Second Approximation)-Case of Field Magnets with Divided Windings-Practical Calculation of Reactions-Comparison with Theoretical Coefficients-Case of Single-Phase AlternatorsConsequences from the Point of View of the Construction of Alternators for Good Regulation.

Chapter II.-Metiods of Testing Alternators According to the Theory of two Reactions............... 270

Method No. I. When the Rigid Coupling of the Two Alternators is possible-Method No. 2. Applicable to a Single Synchronous Machine Operating upon an Actual Conducting System-Analogies between this Method and that of Potier Behrend-Method No. 3-For the Determination of Transverse Reaction (Coefficient L).

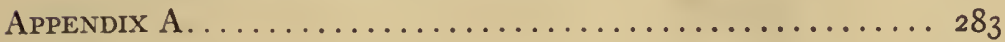

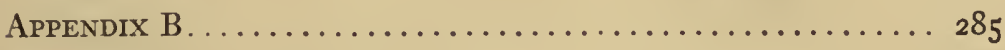

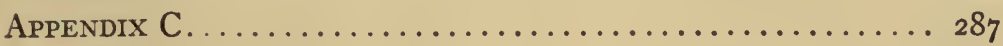

Index. . . . . . . . . . 



\section{INTRODUCTION}

Classification of Singlephase and Polyphase A.C. Motors. An alternating current motor comprises, like any dynamo-electric machine, an inducing magnetic field and its induced circuit, the one turning with respect to the other. But, while the field of D.C. motors is always constant, that of A.C. motors may be either constant, alternating, or revolving, according to whether it be produced by a direct current, an alternating current, or a system of polyphase alternating currents serving to excite windings suitably interlaced. Therefore, as was proposed quite logically, in 1891 , by E. Hospitalier, ${ }^{1}$ A.C. singlephase and polyphase motors can be classified according to the nature of their magnetic fields, into three classes:

(I) Constant field motors.

(2) Alternating field motors.

(3) Revolving field motors.

The first class constitutes the subject-matter of the present book, the second and third classes being reserved for another volume. As will be seen, these three classes each contain singlephase and polyphase motors.

Constant field motors can also be characterized by the fact that the armature-rotation can be maintained at a single speed only, which is synchronous with the alternations of the currents employed. We will therefore call them, more frequently, in accordance with common custom, synchronous motors, in contradistinction to the two other classes, which may be characterized as asynchronous or non-synchronous. For the sake of greater precision, we will apply the latter qualification to motors of the last class only; and since alternating-field motors (with one single exception, which is of little importance) are characterized by the use of a commutator similar to that used with D.C.

${ }^{1}$ Bulletin de la Société francaise de physique, I7. Juillet, I8gr. 
machines, we will give them the more distinctive name of Commutator Motors.

Synchronous Motors. A synchronous motor can be defined as being, merely, an alternator used as a motor. The transmission of power between an A.C. generator and an A.C. motor is, therefore, nothing more than a particular case of the coupling of two alternators in synchronous operation. Indeed, it is precisely through the study of the features of the coupling of alternators in parallel that the occasion presented itself of noting the phenomenon of the reversibility of alternators, that is to say, the possibility of using the same machine both as a motor and a generator, provided that it shall have been previously brought to a speed absolutely equal to that of the generator which supplies it with current.

We can easily understand the possibility of operating such a motor by comparing it to a motor with commutated current. It is known that if the current of a shuttle armature of the Siemens (" $\mathrm{H}$ ') type is commutated at each half revolution, the motor-couple is always in the same direction when the machine is supplied by direct current. In an A.C. system, the same result is obtained without a commutator, because the direction of the supply-current changes at each half revolution, and this effect occurs only when the motion of the motor is synchronous, that is to say when the armature advances the distance of one pole during one alternation of the supply-current.

Although this property was noted as early as 1869 by Wilde, ${ }^{1}$ it passed unnoticed during more than ten years, and it has really been known only since the experiments of J. Hopkinson and Grylls-Adams, at the South Foreland Lighthouse, in 1883 . The Memoir of Hopkinson $^{2}$ (in which, without knowing the work of Wilde, he gives the explanation to which reference will be made later), was epoch-making in the history of alternating currents.

In the South Foreland experiments, the alternators used were three similar de Meritens singlephase alternating current machines, all belt-driven from a common source of power.

1 Wilde, On a Property of the Magneto-electric Current to Control and Render Synchronous the Kolations of the Armature of a Number of Electromagnetic Induction Machines, Philosophical Magazine, January, 1869.

${ }^{2}$ Hopkinson, On the Theory of Alternative Currents, Particularly in Reference to Two Alternate Current Machines Connected to the Same Circuit, Journal of the Society of 'Telegraph Enginecrs, 1884, p. 496, Vol. XIII. See also the paper on The Alternase Current Machine as a Motor, by Grylls-Adams, presented at the same meeting. 
These machines could be easily coupled in parallel, as generators, by bringing them to the same speed before coupling them. The belt being then removed from one of them, it was observed that it continued to run synchronously by the action of the current of its neighbors, and that it could even develop a considerable amount of power, as measured by a friction brake, before losing its synchronism. These experiments were repeated a few years later by Mordey, on a much larger scale, with machines of low inductance presenting a much greater stability of operation and driven by independent prime movers. $\mathrm{He}$ was thus able to demonstrate the synchronizing power of the alternators on the motors or engines driving them, and even to cause one of the latter, with the power shut off, to be dragged by one of the alternators which it was driving. This gives the key to the principles involved in parallel working. He also showed, later, the possibility of accomplishing this coupling with machines connected by means of long lines of high resistance.

Synchronous singlephase motors have two great disadvantages: they are not self-exciting, and they cannot start alone, even without load. Zipernowsky was the first to overcome this difficulty by the expedient of adding a commutator to his motors, which enables them to be started with alternating current, and, after they have attained synchronous speed, to be excited by a portion of the alternating current which they consume.

These motors, manufactured by the firm of Ganz \& Co., had a certain vogue, in consequence of the tests made of them at Frankfort, in I899, by a Technical Commission. The efficiency was satisfactory, being 77 per cent for motors of $1_{5}$ H.P., and 86 per cent for motors of 30 H.P. ${ }^{1}$ This system is no longer used at the present time, except for small powers (I to 5 H.P.).

When the invention of polyphase currents became known, it led naturally to the idea of utilizing them for the transmission of power between two synchronous machines of the same type. Bradley, in America, and Haselwander, in Germany, took out patents, as early as 1887 , the former on a two-phase synchronous motor, and the latter on a three-phase synchronous motor. In both cases the motor was produced by making taps on a Gramme ring and connecting these with insulated rings mounted on the armature-shaft. Non-synchronous motors were only invented in the following year, by Ferraris and by Tesla.

${ }^{1}$ La Lumière Electrique, Vol. XXXII (I889), p. 328. 
It was in 1891 , at the Frankfort Exhibition, that synchronous polyphase motors, with flat ring or Gramme ring armatures, constructed by the firms of Schuckert and of Lahmeyer, and of sizes as large as 50 H.P., were seen, for the first time, alongside the first non-synchronous motors of Dolivo-Dobrowolski and of Brown.

Since that time the principle of synchronous motor operation has been extended to ordinary polyphase alternators, with any winding whatever, stationary or movable, with poles alternating or not; and the only improvements that have been made have been in the means of their excitation or of starting them.

In $1890-1$ Swinburne had had the idea of producing, by means of over-excited synchronous motors, the relatively considerable magnetizing current consumed by his " hedgehog" transformers. Under these conditions the motor played the same role as a condenser. This very interesting property was utilized industrially in 1893 in the BulachOerlikon power-transmission installation, at the suggestion of DolivoDobrowolski; and it was also used in the distributing system at Bockenheim, by Lahmeyer, to compensate for the wattless current of the non-synchronous motors, and even for raising the voltage of the generators. This method has come into extensive use at the present time, especially in the United States.

It constitutes an advantage in favor of synchronous motors, and it has prevented them from disappearing from the commercial field, after the development of motors of revolving field type, which are quite superior to them from other standpoints, notably in regard to starting power. It is desirable to utilize, wherever possible, both these types of motors in distributions of mechanical power. The synchronous polyphase motors are especially useful. They start readily without load, are self-exciting, and have an efficiency equal to that of alternators.

The principal objection to synchronous motors (which is, however, in certain cases, an advantage) is the impossibility of modifying their speed of rotation (without modifying that of the generators). Revolving field motors are superior to them in that respect, but this advantage is obtained at the expense of efficiency. Commutator motors, of which no mention will be made here, are alone capable of running at all speeds, the same as direct current motors; and for this reason they present a certain amount of interest. 
PART I

SYNCHRONOUS MOTORS

CHAPTER I

GENERAL PRINCIPLES OF SYNCHRONOUS MOTORS

Construction. Synchronous motors have the same construction as alternators. The few special features relative to the production of the direct current necessary for their excitation will be treated separately, later. It will be assumed that the reader is already familiar with the general details of construction of alternators.

There are motors having movable armatures and stationary fields, or vice versa, and also motors with revolving iron masses in which all the windings are stationary. These machines are similar to the generators of the same types; for example, Fig. I indicates, diagrammatically, the principle of construc-

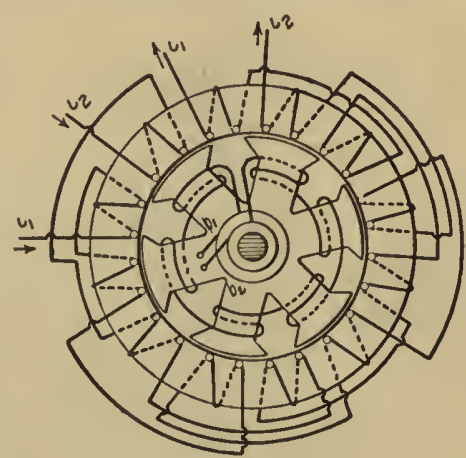

FIG. I. tion of a two-phase synchronous motor, with a ring armature and movable fields, receiving an exciting current through the brushes $b_{1}$ and $b_{2}$.

These motors are designed like generators, the essential condition to be fulfilled being to have a low armature-reaction and powerful inducing fields, in order to obtain good stability.

Although it is more difficult to increase the number of poles for small powers than for large powers, the construction of small 
synchronous motors for ordinary frequencies (40 to 60 cycles) presents no special difficulties, if the speeds corresponding to these frequencies are not objectionable, because these speeds are perfectly allowable so far as centrifugal force is concerned.

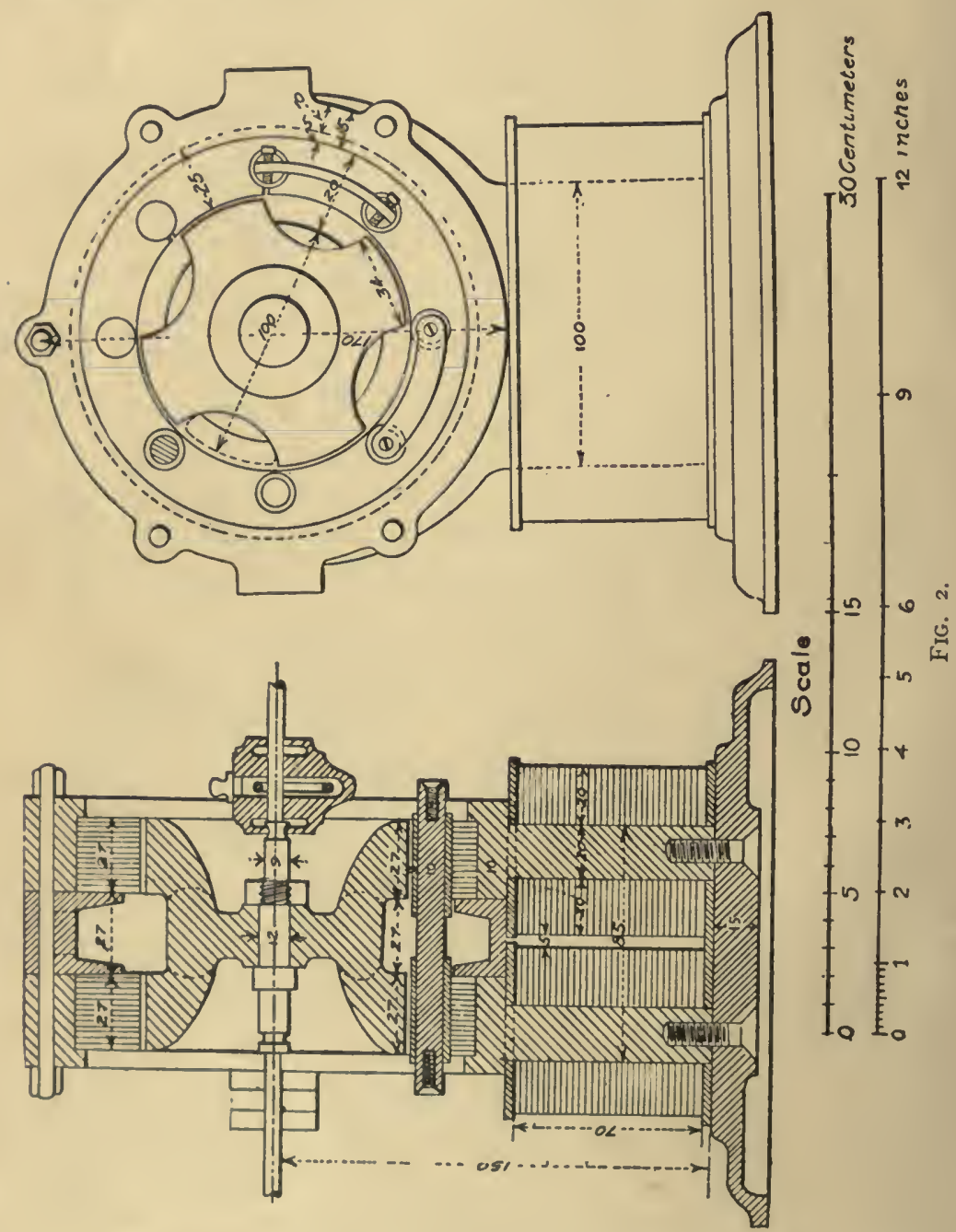

On the other hand, in the construction of small synchronous motors to run at low angular velocities, it is extremely difficult to find space for the numerous conductors and for the exciting or field coils, which 
must produce as many ampere-turns as in the case of large motors. For this reason non-synchronous motors are more convenient for low rotative speeds.

The author has been able, however, to produce motors of low power (a few hundred watts) which have moving iron and have a very high number of poles (as many as 50 for example), by utilizing inductiontype excitation, the magnetic circuit being closed exteriorly, as shown in Fig. 2, in such a way as to allow all the space needed for the exciting coils.

These coils can then be replaced by permanent magnets, thus producing motors whiclı run without excitation, at speeds sufficiently

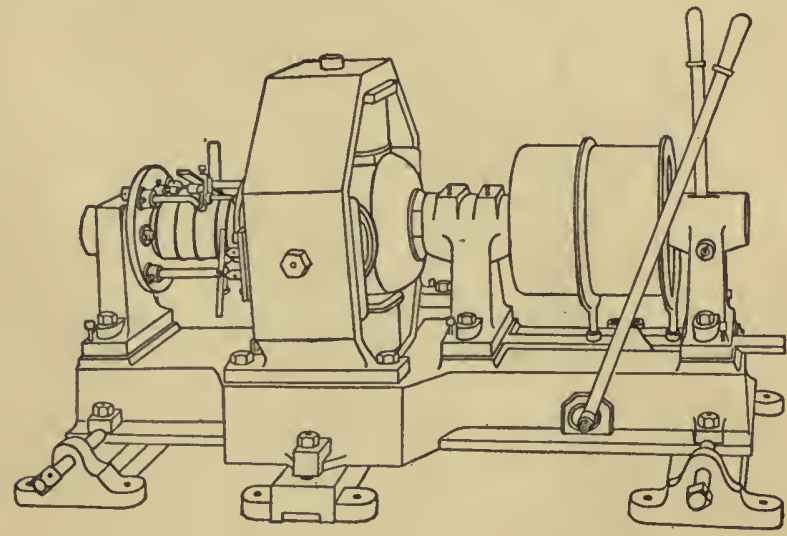

Fig. 3.

low to be synchronized by hand, and which can render useful service, in certain applications, such as for oscillographs. For this purpose the author preferably employs a small horseshoe magnet that is made to revolve around a stationary armature having a number of poles which is a multiple of 6 . It is possible, in this way, to obtain very stable synchronous rotation of a revolving mirror without expending more than $I \frac{1}{2}$ to 2 watts.

Several firms made a specialty of synchronous motors, at an early date, among which we may mention La Société l'Eclairage Electrique in France, and the Fort Wayne Company in America.

One form of motor constructed in France by the Société l'Eclairage Electrique (Figs. 3 and 4), is constructed for polyphase currents or for single-phase currents, for powers ranging from I to I30 H.P. The table on page 5 gives the principal data referring to these matters. 
The efficiencies of the three-phase motors are a little higher than those given for the single-phase motors. The horse-powers given in this table correspond to a frequency of $4^{2}$ periods, but these motors can be also used at frequencies between 40 and 60 periods, and their power then increases with the frequency.

As the table shows, types Nos. I4 to 30 are made with 4 poles, self-exciting. For higher powers, the number of poles increases, and the excitation is obtained by means of a small direct current exciter mounted on the same base. Above type 90 the armature is stationary and the fields turn inside. The fields are of mild cast steel, the armatures being slotted.

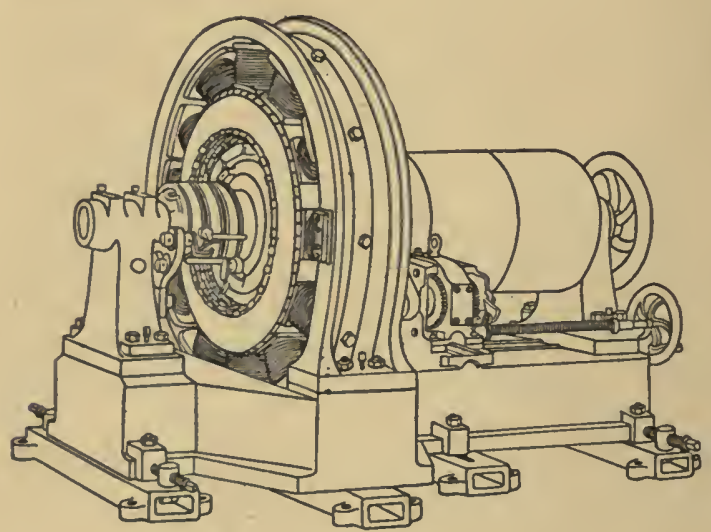

FIG. 4.

As an example of these large motors may be cited several from $50^{\circ}$ to 100 H.P. giving the best of results on the power-transmission system around Grenoble, notably at Voiron, a distance of 30 kilometers from the generating station. Their efficiency is from 90 to 92 per cent. One of these motors even works in parallel with a steam-engine of the same power, and it compensates for the variation of angular velocity of the engine as it passes the dead centers.

All these motors are provided with a clutch and with an idle pulley for starting, as will be explained later. When running, they can undergo considerable variations of load without falling out of step.

Attention should also be called to another interesting type of synchronous motor, the Maurice Leblanc type, which is characterized by the addition of closed circuits in the pole-pieces to insure a perfect damping of oscillations, as will be seen later. 
SINGLE-PHASE AND THREE-PHASE SYNCHRONOUS MOTORS OF THE “SOCIETE L'ECLAIRAGE ELECTRIQUE"

\begin{tabular}{|c|c|c|c|c|c|c|c|c|c|c|c|c|}
\hline \multirow[b]{2}{*}{ 充 } & \multirow[b]{2}{*}{$\begin{array}{l}\dot{y} \\
\text { du } \\
0 \\
\text { Uे } \\
\dot{0} \\
\dot{z}\end{array}$} & \multirow[b]{2}{*}{ 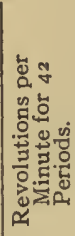 } & \multicolumn{2}{|c|}{ Pulley. } & \multirow[b]{2}{*}{ 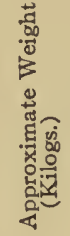 } & \multicolumn{4}{|c|}{ Single Phase. } & \multicolumn{3}{|c|}{ Three Phase. } \\
\hline & & & 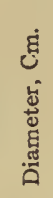 & 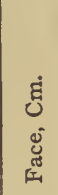 & & 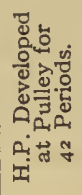 & 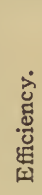 & 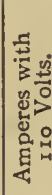 & 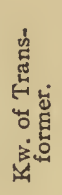 & 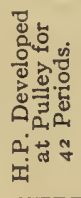 & 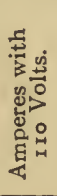 & 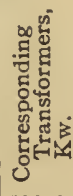 \\
\hline I4 & 4 & I 260 & I4 & 6.5 & I 25 & I & 72 & 9 & I & I $\frac{1}{2}$ & 9 & $I \frac{1}{2}$ \\
\hline I6 & 4 & I 260 & I 6 & 7.5 & I5O & I $\frac{1}{2}$ & 75 & I4 & I $\frac{1}{2}$ & $2 \frac{1}{2}$ & I4 & $2 \frac{1}{2}$ \\
\hline I8 & 4 & I 260 & I8 & 8.5 & 210 & $2 \frac{1}{2}$ & 78 & 23 & $2 \frac{1}{2}$ & $3 \frac{1}{2}$ & 20 & 4 \\
\hline 20 & 4 & I 260 & 20 & 9.5 & 270 & $3 \frac{1}{2}$ & 80 & $3^{2}$ & 4 & 5 & 28 & 5 \\
\hline 22 & 4 & I 260 & 22 & I0. 5 & 375 & 5 & 85 & 45 & 5 & 7 & 37 & $7 \frac{1}{2}$ \\
\hline 24 & 4 & I 260 & 24 & I I & 500 & 7 & 87 & 65 & $7 \frac{1}{2}$ & 9 & 48 & IO \\
\hline 26 & 4 & 1260 & 26 & 12 & 625 & 9 & 88 & 80 & Io & I 2 & 65 & $12 \frac{1}{2}$ \\
\hline 28 & 4 & I 260 & 28 & I 3 & 775 & 12 & 89 & I IO & I $2 \frac{1}{2}$ & I6 & 85 & ${ }^{15}$ \\
\hline 30 & 4 & I 260 & 30 & I4 & 900 & I6 & 90 & I 45 & I5 & 22 & I IO & 25 \\
\hline $4^{2}$ & 6 & 840 & 42 & 17 & $135^{\circ}$ & 22 & 90 & 200 & 25 & 30 & I 55 & 30 \\
\hline $5^{6}$ & 8 & $6_{30}$ & $5^{6}$ & 22 & I $75^{\circ}$ & 30 & 90 & 270 & 3 & 40 & 210 & 40 \\
\hline 70 & IO & 504 & 70 & 29 & 2500 & 40 & $9 \mathrm{I}$ & 360 & 4 & $5^{\circ}$ & 260 & 50 \\
\hline 75 & IO & 504 & 75 & 33 & 3200 & $5^{\circ}$ & $9 I$ & $45^{\circ}$ & 5 & $6_{5}$ & $34^{\circ}$ & 60 \\
\hline $9 \circ$ & I 2 & 420 & 90 & 24 & 3800 & 60 & 91 & $55^{\circ}$ & 60 & 80 & 4 IO & 75 \\
\hline goa & I 2 & 420 & 90 & 29 & 5200 & 75 & $9 \mathrm{I}$ & 675 & 75 & 100 & 500 & 100 \\
\hline I 20 & I6 & 316 & 120 & 38 & 6800 & IOO & $9 I$ & 900 & 100 & 130 & $6_{50}$ & 125 \\
\hline
\end{tabular}

Experimental Properties. As already stated, it is an experimental fact that synchronous motors can only be run after they have first been brought to synchronous speed by some external means. As will be seen later the motors themselves can run indifferently in either direction, but the direction of rotation selected in bringing them to synchronism should be that which is suitable for the brushes of the motor or of the exciter.

Case of Equal Electromotive Forces. Let us suppose that the electromotive forces of the generator and motor are equal, and, to simplify matters, let us take, as generator and motor, two machines whose excitations are regulated to approximately the same value. Let the two machines be driven by belts (Fig. 5); and, when they have attained the same speed, let them be coupled together (experiment of Hopkinson and Grylls-Adams). Let us, moreover, make use of an apparatus of the kind described in Chapter VII, whereby the difference of phase between the two machines may be determained.

It will be noted, in the first place, that as soon as the two machines are brought to the same speed, the current which passes from the one 
to the other practically disappears. Moreover, the "phases are identical," i.e., the poles of like polarity pass at the same time in front of the corresponding portions of the two armatures.

The induced E.M.F.'s between the corresponding terminals $a$, $b$, and $A, B$, are therefore in unison. If we measure them, on the contrary, in the direction in which they appear, by following the circuit $a b, B A$, it will be found that they are exactly opposed to each other.

Let us now suppose the belt of one of the two machines to be removed. This machine will continue to/turn at the same speed, but it gives indication of a certain very slight delay or falling behind, technically termed "lag," with respect to the other machine. Moreover, the current in the circuit now becomes appreciable.

If a brake be placed on the pulley and if the load be gradually increased in such a way as to increase the mechanical power produced

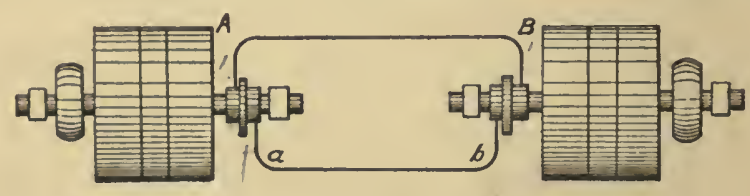

FIG. 5.

by the motor, the "lag" of the motor will be seen to increase at the same time as the current.

When this lag approaches a quarter of a period, i.e., half an interpolar space, the machine slows up all at once and stops as if held fast by the brake. We thell say that it is "stalled," or "out of synchronism," or "out of step." The current in the circuit rises to a very high value as soon as the machine falls out of synchronism; and it becomes approximately equal to the short-circuit current in the circuit when the machine is stopped. In order to avoid accidents, it is necessary to introduce fuses in the circuit, or to provide some automatic disconnecting device, which will prevent the excessive load.

It is seen that what characterizes the synchronous motor is the increase of phase-lag with the load and the "stalling" of the motor or its falling out of step beyond a certain maximum load.

In a good motor, the limiting load should amount to at least 1.5 times, or, better, to twice the normal load. This limit is guaranteed by most makers of synchronous motors.

On the other hand, if the motor is run by a belt in such a wily as to give it a "lead in phase," with respect to the machine or the circuit 
which supplies it with current, it can be found, by wattmeter measurements, that this power changes in sign, i.e., the motor acts as a brake and returns energy to the circuit instead of receiving it therefrom.

The phenomena become more complicated still on varying the E.M.F. of the motor or of the generator.

Case of Unequal Electromotive Forces. An interesting and characteristic property of synchronous alternating current motors, and which distinguishes them absolutely from direct current motors or from alternating current motors having commutators, is that they can be excited so as to give a voltage greater than that of the supply-circuit. For example, it is possible to feed, from a Iro-volt circuit, a motor which, driven by belt at the same speed, produces an E.M.F. of $I 20$ to I 50 volts at its terminals. But, if the E.M.F.'s are thus unequal, the current passing between the generator and the motor, when the latter is running without load, can, instead of being inappreciable, attain a considerable value. Likewise, when the motor is running with load, the current is greater than that which corresponds to the work to be done. The same effects are produced when, instead of giving to the motor an excessive excitation, it is given an insufficient induced E.M.F. It is then observed, if the machines are alike, that the potential difference at the terminals assumes a third value, which is the mean of the two E.M.F.'s involved.

In both cases, the greater the inequality between the two E.M.F.'s the more the current measured will increase, by the change of excitation. If we plot a diagram, taking, as abscissa, the values of the excitation of one of the machines, and, as ordinates, the current passing through the circuit, the curve of variation of the latter, as a function of the former, has the form of a $V$ more or less rounded at the bottom (Fig. 6): This form persists, although it may be less marked, when a constant load is placed on the brake. At the same time that the current increases, by reason of an inequality of the E.M.F.'s, it can be noted, by means of an apparatus for indicating phase-difference, that the current undergoes a change of phase, either forward or backward, with respect to the E.M.F. of the motor. This can be expressed in another way by saying that the machine consumes or produces wattless current, ${ }^{1}$ i.e., current which is "out of phase," being $\frac{\pi}{2}$ behind or ahead of the E.M.F. This "wattless" current, which has the effect of increasing the "apparent" current, is thus named because it produces no work, the load on the brake remaining constant, by hypothesis.

1 See note at bottom of page 42 . 
The effect of this wattless current is, therefore, to produce, in the motor, a supplemental positive or negative E.M.F., which adds itself to its own induced E.M.F., in such a way as to produce, at the terminals, a difference of potential equal to that of the generator. We can conclude from this, without further argument, that when the motor generates an E.M.F. which is too low, the current of the generator tends to over-excite it and that, in the contrary case, it tends to under-excite it.

The action of the current on the generator itself produces inverse effects.

The effects are more complicated still when resistances or inductances are added in the circuit between the machines, with the general

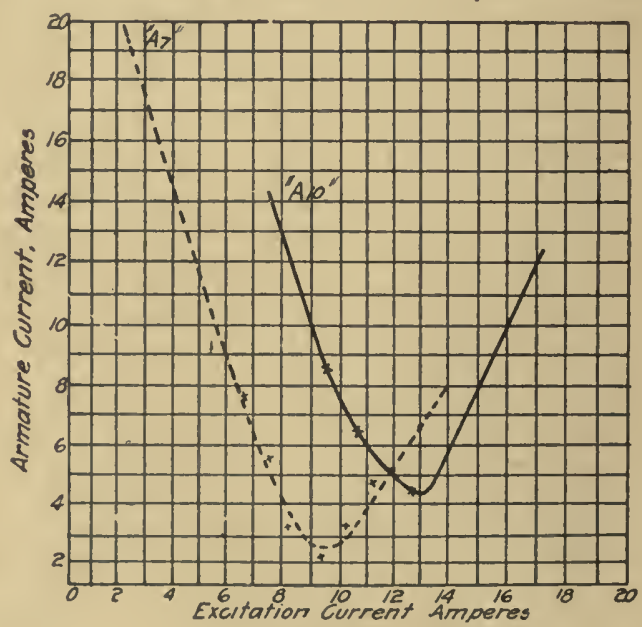

FIG. 6.

effect of lowering the voltage. Synchronous operation remains possible, nevertheless, even when the resistance attains high values. ${ }^{1}$

When the circuit includes reactance it is observed that, by overexciting the motor, the voltage will be raised at its terminals, and even at the terminals of the generator, so as to attain values which are higher than the E.M.F. of the generator, measured with open circuit. On the contrary, by under-exciting the motor, it is possible to produce increasing and rapidly exaggerated voltage-drop along the whole line.

'When the resistance is high, say 75 per cent, it may be necessary to add some external reactance, so as to cause the current to lag more behind the cross E.M.F.-(E. J. B.) 
An over-excited synchronous motor, connected to the terminals of an alternator having excessive armature-reaction, can even replace the excitation of the latter. It is observed, indeed, that on suppressing this excitation, the generator continues to run the motor, and furnishes the normal voltage at its terminals; but it can develop only little power. An over-excited motor thus produces an indirect self-excitation which is equivalent to that obtainable from a condenser. There is, in other respects, a complete analogy of effects between the two forms of apparatus.

These experimental results are much too complex to be studied more in detail here. They can be discussed more satisfactorily later, in connection with the theory of these motors and their applications.

Elementary Explanation of Polyphase Synchronous Motors. If we turn our attention, first, to polyphase synchronous motors, the explanation of the phenomena just described is made easy by the consideration of revolving magnetic fields.

For the sake of brevity we will adopt the terms "rotor" and "stator" to designate the movable and fixed portions, respectively, of the motor, in accordance with the terminology of Professor S. P. Thompson.

Let us take, as an example, a motor having two pairs of pole-pieces, in which the inductive circuit is of movable form (rotor) and the induced is circuit of stationary form (stator).

In the ordinary form of polyphase alternators, the rotor will consist of a crown of iron cores, with protruding poles excited by coils receiving direct current from a separate exciter. The stator, on the other hand, will consist of a circular core of laminated iron having some induced windings disposed in notches or slots in such a manner that the wires in the successive slots shall have alternating currents of different phase passing through them. If we suppose, for example, that we have a winding for four poles and for six phases (three slots per pole) such as is shown in Fig. 7, the wires in the six slots which cover two poles of the stator, as we follow along the periphery of the latter, will have, passing through them, six currents ${ }^{1}$ which are out of phase with respect to each other by $\frac{1}{6}$ of a period, and which can be represented by the equations

$$
\begin{aligned}
& i_{1}=I_{0} \sin \omega t \\
& i_{2}=I_{0} \sin \left(\omega t-\frac{2 \pi}{6}\right)
\end{aligned}
$$

${ }^{1}$ In reality, the six phases are supplied by three-phase currents only; the windings which are of exactly opposite phases being connected in series, with reversed connections. 


$$
\begin{aligned}
& i_{3}=I_{0} \sin \left(\omega t-2 \frac{2 \pi}{6}\right) ; \\
& i_{4}=I_{0} \sin \left(\omega t-3 \frac{2 \pi}{6}\right) ; \\
& i_{5}=I_{0} \sin \left(\omega t-4 \frac{2 \pi}{6}\right) ; \\
& i_{6}=I_{0} \sin \left(\omega t-5 \frac{2 \pi}{6}\right) ;
\end{aligned}
$$

in which

$$
\omega=\frac{2 \pi}{T} \text {. }
$$

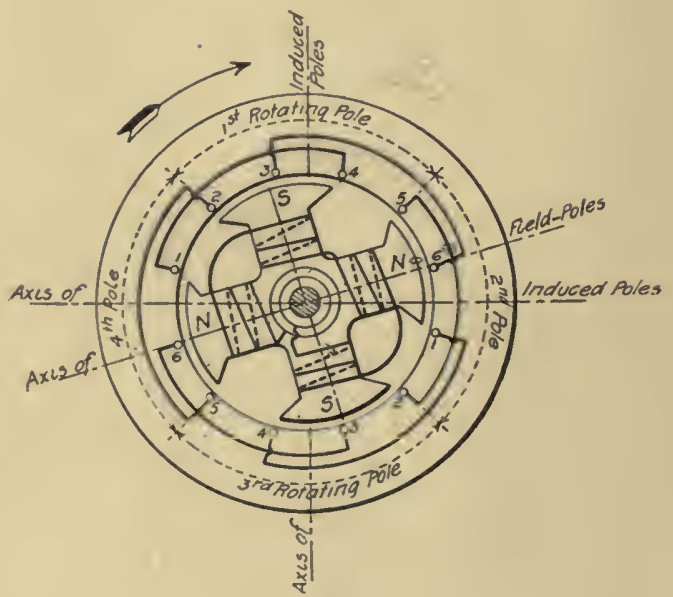

FIG. 7 .

$T$ being the common duration of the period of the alternating currents considered, $I_{0}$ their common amplitude (i.e., maximum value), and $i_{1}, i_{2}, i_{3}, i_{4}, i_{5}, i_{6}$, being the currents in the slots I, $2,3,4,5,6$.

It will be seen that, at every one-sixth of a period, the currents in the stator resume the same values, but the latter are displaced onesixth of the width of a double field ( 2 poles) in the direction in which the currents succeed each other along the stator. Therefore, the axes radiating from the magnetic fields produced by the windings of the stator displace themselves around this stator with an angular velocity;

$$
\alpha=\frac{\omega}{p}=\frac{2 \pi}{p T},
$$

corresponding to a number of turns $=\frac{60}{p T}$ per minute. 
The magnetic strength of these fields can be considered practically constant inasmuch as it also resumes the same value at every one-sixth of a period (although it may, in the intervals, undergo slight variations which are dampened by the hysteresis and the eddy currents produced in the pole-pieces of the rotor). Therefore, even though the armature (stator) may be stationary, the result is the same as if it had rervolving poles which attract or repel the poles of the field (rotor) and we can, henceforth, reason as if we were dealing with the attractions of two systems of magnets presenting the same number of poles which are alternately north and south in polarity. (Fig. 8.) The poles of unlike polarities, of the two systems, attract each other; the others repel each other. Therefore, when at rest, the poles of unlike polarity will always face each other. If the external magnets begin to rotate slowly, starting from rest, they will drag with them the stationary magnets, whose poles tend to remain opposite the poles of unlike polarity. (This result may be obtained by supplying the motor with current obtained from a generator which

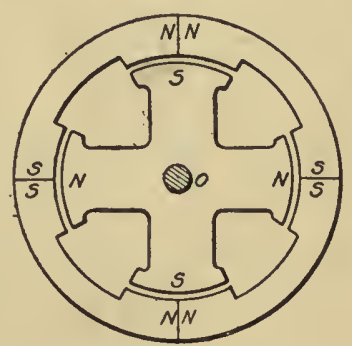

FIG. 8. is started from rest and, consequently, gives polyphase currents of increasing frequency.)

The attractions can only be concordant and continuous when the two systems turn at the same speed; which explains the necessity of synchronism. Otherwise, there would only be successive attractions and repulsions which would neutralize each other.

The stability of synchronous operation is also easily demonstrated by considering the moment of the motor-couple (i.e., the torque). If the poles of the rotor remain opposite the revolving poles of the stator, the attractions produced are directed radially and consequently they produce a motor-couple or torque which is equal to zero. If, on the contrary, for any reason whatever, the rotor loses or gains speed, some tangential attractions or repulsions will appear, whose resultants tend to bring back the opposite poles of the rotor into coincidence with the poles of unlike polarity of the stator, so long as the poles of the rotor remain near these, because the attractions of unlike poles and the repulsions of like poles act in the same direction; but if the difference in phase amounts to one interpolar space, the poles of like sign of the rotor and stator will be opposite cach other, the motor-couple or torque 
will become zero, and will then change sign if the difference in phase increases. By reason of the symmetrical construction of the motor the torque will have points of maximum and minimum value at equal distances between the points of zero-value, i.e., in the positions where the poles of the rotor are midway between the poles of the stator.

To sum up, taking as absciss $x$ the difference of phase $l$ of the poles of the rotor, expressed in terms of the interpolar space $L$, and taking as ordinates the torque $C$, the representative curve will take the form shown herewith (Fig. 9), the magnetic strength at the armature-poles being supposed constant, i.e., assuming the currents that produce this magnetic flux to be constant.

The machine will have stable operation for the difference of phase comprised between the two maximum points $B$ and $C$ (the positive

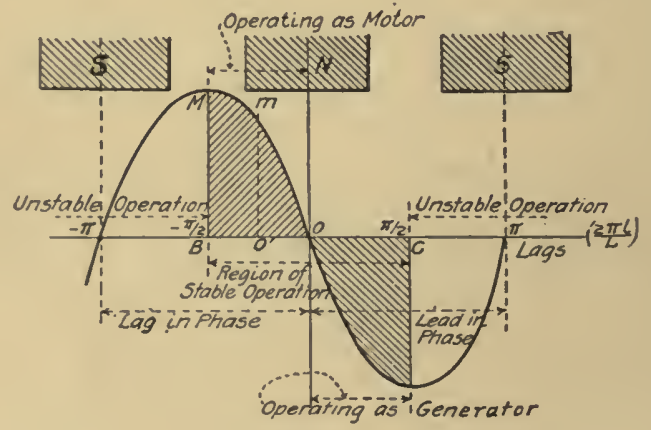

Fig. 9.

maximum being due to a lag, and the negative maximum being due to a lead), because every accidental advance (or lag) is corrected of itself by a contrary variation of the torque. If the rotor lags, for example, in consequence of a passive mechanical resistance, the increase in torque compensates for this resistance.

When the motor is running without load, its condition corresponds to the position $O$, at which there is no phase-difference. When the motor is loaded, i.e., whenever mechanical resistance is applied to the shaft, the position of the poles of the rotor changes in phase and comes to a point $O^{\prime}$, such that the couple $O^{\prime} m$ shall balance the resisting couple. If the resisting couple is greater than the maximum torque, the machine can no longer run; and even for positions of $m$ which are a little below $M$, the machine will fall out of step, in consequence of unavoidable oscillations. 
On the other hand, to obtain a difference of phase ahead, between $O$ and $C$, it is necessary to apply to the shaft an effort in the direction of rotation, i.e., it is necessary to apply to the shaft a certain amount of propelling power which must, evidently, be transformed into electrical energy.

The armature current has been supposed constant. In practice, it is the voltage of the supply-circuit which is constant, at its terminals; and the question is thus complicated by the spontateous variation of the current with the variation in phase-difference. This variation, itself, depends on the ratio of the induced E.M.F. of the motor to the voltage applied at its terminals.

In fact, as they displace themselves before the armature at the synchronous speed, the poles of the inducing field induce in the windings counter E.M.F.'s. which are of the same order and magnitude as the voltage at the terminals. If, mentally, we locate the E.M.F.'s. in the wires which are placed in the slots, we perceive readily that the E.M.F. in each slot varies periodically and passes through a maximum at the moment when the middle of a revolving pole comes in line with the slot. 'The axes of maximum values of the induced E.M.F.'s therefore coincide with the axes of the inducing poles, and revolve with them. If the currents were in phase with the E.M.F.'s, they would give rise to revolving fields whose axes would be retarded in phase by an amount equal to half the width of a pole, since each conductor forms a coil with a conductor similarly placed, but in the contrary direction, under the next pole.

But we must take into account the voltage at the terminals, with which the induced E.M.F. combines, and also the self-induction of the machine, which throws the current out of phase by a quarter of a period, i.e., half an interpolar space. Therefore the question can only be treated with precision by calculation, as will be seen later. From the qualitative point of view, the result differs but slightly from the preceding result. The form of the curve of torque remains analogous to that of Fig. 9, but it is no longer so symmetrical, and the lags $O C$ and $O B$, which determine the limits of stability, take a value, $r$, which is a little lower than $\frac{\pi}{2}$, and which is defined by the relation

$$
\tan \gamma=\frac{\omega L}{R}
$$

$\omega L$ and $R$ being, respectively, the reactance and the resistance of the armature circuit. 
It will be observed that, when the current is in phase with the induced E.M.F., the magneto-motive force of the armature-reaction has no action on the inducing field, and can only produce a transverse distortion of the field; while, on the other hand, when the current is out of phase one-fourth of a period in advance, or behind, the M.M.F. is directly opposed to, or coincident with, that of the field. With regard to the sign, it can be easily seen that a phase-difference of $\frac{\pi}{2}$ in advance of the induced E.M.F. produces a magnetizing reaction which is the same as in a generator, and that a phase-difference of $\frac{\pi}{2}$ behind produces a demagnetizing reaction. It must not be forgotten, however, that the internal E.M.F. is opposed to the external E.M.F. and that the lag and lead are therefore transposed if they are referred to the latter.

Elementary Explanation of Single-Phase Synchronous Motors. The phenomena are more complicated in single-phase motors. The same explanation may nevertheless be retained by means of a simple artifice of reasoning.

The coils of the armature-ivinding, being excited by a single alternating current (Fig. Io), produce poles which no longer revolve, but are stationary. These poles are alternately positive and negative, and have a magnetic flux which varies periodically like the current that produces it. There is, therefore, no tendency to rotation; and the motor can only be put in operation by external means, as already seen. But we may suppose it brought previously to synchronism.

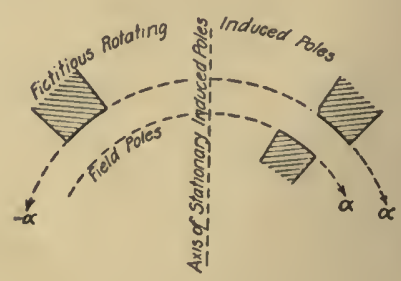

FIG. IC.

M. Maurice Leblanc has enunciated a theorem which is an electrical analogue of the following well-known optical theorem: A vibration of luminiferous ether polarized rectilinearly may be replaced by two circularly polarized vibrations of contrary sign having the same frequency and having amplitudes equal to the half amplitude of the rectilinear vibration.

According to M. Leblanc's theorem, an alternating stationary magnetic field may be replaced by two fields revolving in contrary directions, each having a flux half as large, and having equal velocities, such that they advance a distance equal to that of two poles during a 
single period. The fields turning in the same direction as the inducing poles will have the same angular velocity $\alpha$, as the latter, and will drag them in very much the same way as in polyphase motors. On the other hand, the fields which turn in the opposite direction will have a relative velocity, $2 \alpha$, which is contrary to and double that of the field-poles, so that their attracting or repelling actions, since they succeed each other in inverse directions, will produce no resisting torque. These reversed revolving fields will give rise only to supplemental losses by hysteresis and by eddy currents.

By this simple analysis (which is, in reality, only approximate) the operation of single-phase motors can, it is seen, be discussed and explained in the same way as that of polyphase motors.

It has been supposed, in what precedes, that the armature is stationary and the field movable. In the contrary case the explanation is the same if we consider the relative velocities of the two portions, but the fields displace themselves only with respect to the armature and therefore remain stationary in space the same as the field-poles.

Equations of Synchronous Motors. Analytical Theory. We have just examined the phenomena of synchronous motors from a physical point of view. We shall now represent them analytically, according to the theory first expounded by Dr. J. Hopkinson, but with a few modifications in form. We shall suppose with him that the E.M.F.'s and currents follow the sinusoidal law, and that the reactances of the machine are constant.

Let us suppose, then, a single-phase A.C. generator and motor, defined by their induced E.M.F.'s, their resistances, and their mean inductances, which are all supposed constant.

Let $\quad T=$ the duration of the period;

$\omega=\frac{2 \pi}{T}=$ the speed of pulsation of the currents;

$e_{1}$ and $e_{2}=$ the instantaneous values of the generator and motor E.M.F.'s respectively, at the instant $t$;

$E_{1}$ and $E_{2}=$ the effective values equal to the amplitudes of the sinefunctions, i.e., the maximum value, divided by $\sqrt{2}$;

$\frac{t_{0}}{T}=$ the phase-difference between $e_{1}$ and $e_{2}$;

$\theta=$ the angle of lag (phase-difference) corresponding to

$$
\theta=2 \pi \frac{t_{0}}{T}
$$


$R$ and $L=$ the resistance and inductance, respectively, of the total circuit, of the two machines;

$i=$ the instantaneous value of the current;

$I=$ the effective value of the current, equal to the maximum value divided by $\sqrt{ } \overline{2}$.

Let us suppose the conditions of stability to be unknown and let us seek to ascertain how two alternators connected in series will operate.

The two sine-functions of the E.M.F. represented by the curves $e_{1}$ and $e_{2}$ in Fig. II, may be formulated by the equations,

$$
\begin{aligned}
& e_{1}=E_{1} \sqrt{2} \sin \left(\omega t+\frac{\theta}{2}\right) ; \\
& e_{2}=E_{2} \sqrt{2} \sin \left(\omega t-\frac{\theta}{2}\right) ;
\end{aligned}
$$

in which $\theta$ designates the angular distance between the actual position of $e_{1}$ and the position of opposition of $e_{2}$.

The E.M.F. which is acting in the circuit is equal to the algebraical sum of the opposing E.M.F.'s.

$$
\varepsilon=e_{1}+e_{2}=E_{1} \sqrt{2} \sin \left(\omega t+\frac{\theta}{2}\right)-E_{2} \sqrt{2} \sin \left(\omega t-\frac{\theta}{2}\right) .
$$

From this the current, $i$, may be deduced, by the well-known differential equation,

$$
R i+L \frac{d i}{d t}=\varepsilon=E_{1} \sqrt{2} \sin \left(\omega t+\frac{\theta}{2}\right)-E_{2} \sqrt{2} \sin \left(\omega t-\frac{\theta}{2}\right) .
$$

In this equation let $i=X \sin \omega t+Y \cos \omega t$.

If this value be substituted in the equation, the values of $X$ and $Y$ can be determined by making the coefficients of the sine-terms and of the cosine-terms successively equal to zero. We can then obtain, by differentiation, substitution, etc., ${ }^{1}$ the following value for $i$ :

$$
i=\frac{R}{R^{2}+\omega^{2} L^{2}}\left\{\begin{array}{r}
E_{1} \sqrt{2}\left[\sin \left(\omega t+\frac{\theta}{2}\right)-\frac{\omega L}{R} \cos \left(\omega t+\frac{\theta}{2}\right)\right] \\
\left.-E_{2} \sqrt{2}\left[\sin \left(\omega t-\frac{\theta}{2}\right)-\frac{\omega L}{R} \cos \left(\omega t-\frac{\theta}{2}\right)\right]\right]
\end{array}\right\} \text {. }
$$

${ }^{1}$ See Appendix $A$. 
This may also be written,

$$
i=\sqrt{\frac{E_{1}^{2}+E_{2}^{2}-2}{R^{2}+\omega_{1}^{2} L^{2} \cos \theta}} \sin (\omega t-\beta-\gamma) ;
$$

wherein

$$
\begin{aligned}
& \tan \beta=\frac{E_{2}-E_{1}}{E_{2}+E_{1}} \tan \theta ; \\
& \tan \gamma=\frac{\omega L}{R} .
\end{aligned}
$$

In this equation $\beta=$ the phase-angle of the resultant E.M.F. and $\gamma=$ the supplemental phase-difference of the current measured from this E.M.F.

In the simple particular case where $E_{1}=E_{2}$ this expression reduces to

$$
i=2 E \sqrt{2} \frac{\sin \frac{\theta}{2}}{R^{2}+\omega^{2} L^{2}}[R \cos \omega t+\omega L \sin \omega t] ;
$$

or, since

$$
\frac{R}{\sqrt{R^{2}+\omega^{2} L^{2}}}=\cos \gamma ; \quad \frac{\omega L}{\sqrt{R+\omega^{2} L^{2}}}=\sin \gamma
$$

we will have

$$
i=\frac{2 E \sqrt{2} \sin \frac{\theta}{2} \cos (\omega t-\gamma)}{\sqrt{R^{2}+\omega^{2} L^{2}}} ;
$$

i.e., the current will have the effective value

$$
I_{\text {eff. }}=2 E \sqrt{2} \sin \left(\frac{\theta}{2}\right) \frac{\mathrm{I}}{\sqrt{R^{2}+\omega^{2} L^{2}}}
$$

and will be out of phase by the angle $r$ with respect to the resultant E.M.F., which is itself out of phase $\frac{\pi}{2}$ with respect to the mean of $e_{1}$ and $e_{2}$. This result is easily interpreted in Fig. I I, by drawing the resultant curve $e_{1}+e_{2}$, obtained by taking the difference of the ordinates of the first two curves. It will be seen that the curve has actually a phase-difference equal to $\frac{\pi}{2}$ with regard to the mean of $e_{1}$ and $e_{2}$, and 
that it increases with the phase-difference of $e_{2}$ with respect to $e_{1}$. In consequence of the lag, $\gamma$, of the current, measured with respect to this resultant E.M.F. (when $\gamma$ is near $\frac{\pi}{2}$ in value), it will be seen that the current is approximately in phase with this mean value; it would be completely so if there were no resistance-losses.

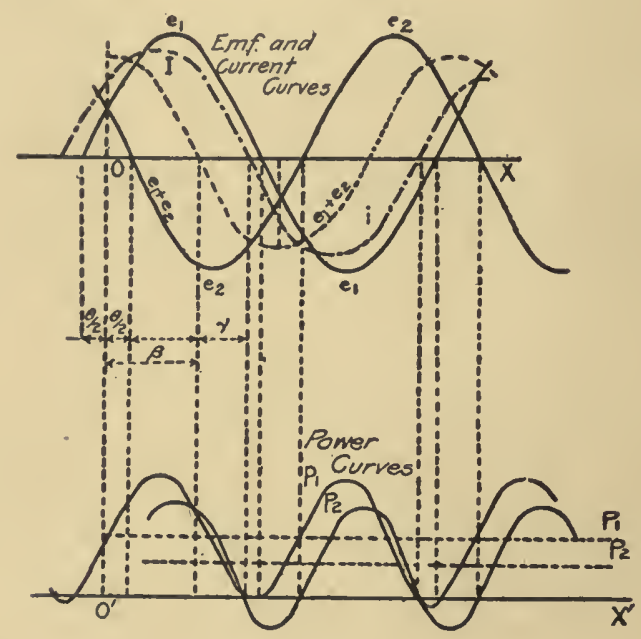

Fig. Ir.

The power-outputs of the two machines will be obtained by multiplying the instantaneous current $i$ by the E.M.F.'s. $e_{1}$ and $e_{2}$. For example, in the case where $E_{1}=E_{2}$, we have

$$
\begin{aligned}
p_{1} & =\frac{{ }_{4} E^{2}}{\sqrt{R^{2}+\omega^{2} L^{2}}} \sin \frac{\theta}{2} \cos (\omega t-\gamma) \sin \left(\omega t+\frac{\theta}{2}\right) \\
& =\frac{{ }_{2} E^{2}}{\sqrt{R^{2}+\omega^{2} L_{J^{2}}}} \sin \frac{\theta}{2}\left[\sin \left(\gamma+\frac{\theta}{2}\right)+\sin \left(2 \omega t-\gamma+\frac{\theta}{2}\right)\right] .
\end{aligned}
$$

Likewise, on multiplying by $-e_{2}$, we will have

$$
p_{2}=\frac{+2 E^{2}}{\sqrt{R^{2}+\omega^{2} I_{0}^{2}}} \sin \frac{0}{2}\left[\sin \left(\gamma-\frac{\theta}{2}\right)+\sin \left(2 \omega t-\gamma-\frac{0}{2}\right)\right] \text {. }
$$

These equations show that the power is not constant, in either case, but pulsating, i.c., it presents variations of frequency $=2 T$, as represented in Fig. I 1. 
These variations constitute sine-functions having pulsations twice as rapid as those of the current, which have for their axes the horizontal lines $\left(P_{1}, P_{2},\right)$ corresponding to the mean powers given by the first terms within the brackets in the following equations:

$$
\begin{aligned}
P_{1} & =\frac{2 E^{2}}{\sqrt{R^{2}+\omega^{2} L^{2}}} \sin \frac{0}{2} \sin \left(\gamma+\frac{\theta}{2}\right) \\
& =\frac{E^{2}}{\sqrt{R^{2}+\omega^{2} L^{2}}}[-\cos (\gamma+\theta)+\cos \gamma] ; \\
P_{2} & =\frac{2 E^{2}}{\sqrt{R^{2}+\omega^{2} L^{2}}} \sin \frac{0}{2} \sin \left(\gamma-\frac{\theta}{2}\right) \\
& =\frac{E^{2}}{\sqrt{R^{2}+\omega^{2} L^{2}}}[-\cos \gamma+\cos (\gamma-\theta)] .
\end{aligned}
$$

The very small difference between $P_{1}$ and $P_{2}$ represents the loss by resistance (Joule effect). The axis of the curve $P_{1}$ is therefore a little more above the axis of zero power than the axis of symmetry of the curve $P_{2}$ is below it.

The torque could be obtained, in each case, by dividing the power by the angular velocity. These expressions show that the current increases with $\theta$ until $\theta$ equals $\pi$, but the torques, which equal zero so long as the lag $\theta=$ zero, increase with 0 only until the value $\theta=r$; and they then decrease.

Stability will, therefore, exist only with $\theta<\gamma$ having for its axis the exact opposition of E.M.F.'s.

The solution, in the case where $E_{1}$ is different from $E_{2}$, will be obtained in an analogous manner, by forming the products $e_{1} i-e_{2} i$; and it would still give pulsating values for $p_{1}$ and $p_{2}$; but, since these caculations are uselessly complicated, we will pass them by and turn to more simple methods.

Case of Symmetrical Polyphase Motors. In the case of polyphase motors the same considerations and equations remain applicable to each of the symmetrical circuits, if only care be taken to include, in the self-induction of these circuits, their mutual induction effects. By reason of symmetry itself, the result is obtained by a simple increase of the coefficient, $L$, in a ratio which depends on the number of phases in the machine.

The currents are, in general, approximately equal in effective values in the different circuits, if there are no defects in construction. It 
is sufficient, therefore, to apply the reasoning to one of the circuits only, and to multiply the power by the number of circuits.

It should be noted, moreover, that, in these motors, the pulsations of the power derived from the different circuits, occurring at different intervals, compensate each other, from the standpoint of the total power, which becomes constant. This is easily shown by taking the sum of the powers. For example, in a three-phase motor, the pulsations at the instant $a \omega t$ will have the form

$$
\begin{aligned}
& A \sin \left(2 \omega t-\gamma-\frac{\theta}{2}\right) ; \\
& A \sin \left(2 \omega t-\gamma-\frac{\theta}{2}-\frac{2 \pi}{3}\right) ; \\
& A \sin \left(2 \omega t-\gamma-\frac{\theta}{2}-\frac{4 \pi}{3}\right) .
\end{aligned}
$$

It is known that the sum of the sines of three angles differing from "each other by $120^{\circ}$ is identically equal to zero. Therefore the "resultant" pulsation is equal to zero. The same thing would be true for any number whatever of equidistant and symmetrical phases.

The result is that the torque is constant (to a sufficient degree of approximation for this theory, which neglects the higher harmonics of the field-distortions), whereas, in a single-phase motor, it undergoes heavy periodical variations. In the latter case, the inertia of the armature plays the rôle of a flywheel storing and restoring energy twice during each period; but if the inertia is insufficient, the velocity of the armature will experience slight variations which will greatly interfere with the stability of operation. Polyphase motors are, in this respect, superior to single-phase motors; they are also superior to them in being lighter for a given output (less weight per kilo-volt-ampere) and also in having higher efficiency.

Graphical Representation of Operative Conditions. Blakesley's Method. Mr. Blakesley was the first to apply Fresnel's method of vectors to the study of alternating currents. ${ }^{1}$

The following principles constitute the basis of Fresnel's method:

r. Any sinusoidal function can be represented in magnitude and in phase by a vector, or a segment of a right line, whose length is proportional to the amplitude of the function and whose phase is measured by an angle reckoned from some other vector serving as a point of origin.

' Blakesley, Alternating Currents of Electricity, 1885 
2. The addition or subtraction of sinusoidal functions may be made on the graph by a geometrical addition or subtraction of the vectors of these functions.

3. The mean product of two functions is equal to the work done by one of the vectors on the other, i.e. it is equal to the area of the triangle constructed on vectors which are equal to the effective values of the variables, or to half this area when the triangle is constructed with the amplitudes of the sinusoidal functions.

This method, with which the reader is supposed to be familiar, has been applied by Blakesley to the problem of synchronous motors in the following manner:

Let us represent the amplitudes of the E.M.F.'s. of the generator and of the motor, $E_{1} \sqrt{2}_{2}^{-}$, and $E_{2} \sqrt{ }_{2}^{-}$, respectively, by means of two vectors, $O A$ and $O B$, (Fig. I2), having between them the angle $\pi+\theta$. These E.M.F.'s are approximately opposed to each other, as we have seen in what precedes.

Let $O B^{\prime}$ be equal but opposed to $O B$. The E.M.F. $\varepsilon \sqrt{2}$, which is the resultant of $E_{1} \sqrt{2}$ and $E_{2} \sqrt{2}$, will be represented by the vector $B^{\prime} A$ which is equal to the resultant of $O A$ and $O B$.

Let us again designate by $r$ the phase-angle between the current and the resultant E.M.F. defined by the relation

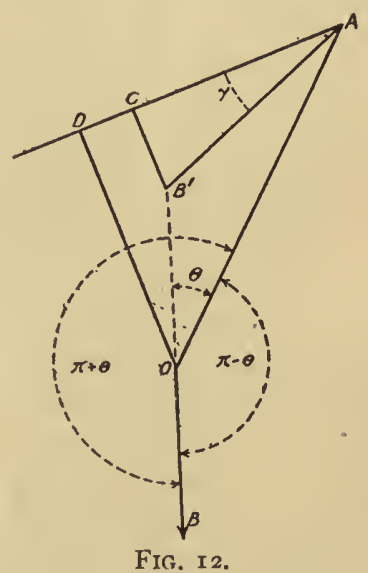

$$
\tan \gamma=\frac{\omega L}{R}
$$

and let this angle be drawn with respect to the point $A$. The direction $C A$ will represent the current which is in phase. Let $C A$ be the projection of $B^{\prime} A$. We will have

$$
\overline{C A}=\overline{B^{\prime} A} \cos \gamma=\varepsilon \sqrt{2} \cos \gamma=\frac{\varepsilon \sqrt{2}}{\sqrt{R^{2}+\omega^{2} L^{2}}} \times R,
$$

or

$$
I \sqrt{2}=\frac{\overline{C A}}{R} .
$$

The length of $C A$ is therefore proportional to $I$. 
The power supplied by the generator to the motor will be

$$
\begin{aligned}
P_{1} & =E_{1} I \cos O A D \\
& =\frac{\overline{D A} \times \overline{C A}}{2 R} .
\end{aligned}
$$

In like manner, the electric power consumed by the motor will be

$$
P_{2}=\frac{\overline{D C} \times \overline{C A}}{2 R} \text {. }
$$

The power lost by ohmic resistance (Joule effect) is equal to the difference, or to

$$
\frac{C A \times C A}{2 R} \text {. }
$$

The diagram therefore determines, by the measurement of simple lines or areas, the current strength and the power of each of the two machines, for each value of the phase-angle.

Let us suppose that the vector $O A$ (Fig. I3) is stationary, and that

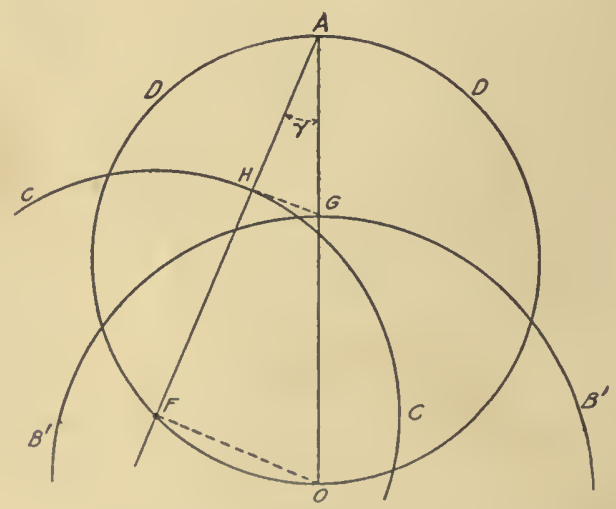

FIG. I3.

the phase-angle, 0 , varies. The point $B^{\prime}$ will describe a circle of radius $O B^{\prime}$; the point $D$ describes a circle constructed on $O A$ as a diameter; finally, it is easily seen that the point $C$ describes a third circle having for its center and for its radius the projections of $O$ and of $O G$ on a right line, $A F$, making the angle $r$ with $O A$. These three circles having been drawn, the conditions of operation can be followed on the diagram. Mr. Blakesley has thus determined the limits of stability and the maximum electrical efficiency. 
But even for easy questions the use of this diagram is too complicated; the same thing is true of the diagrams devised by Kapp, ${ }^{1}$ after Blakesley, and based on the same principles. The author has also given $^{2}$ a more convenient method of representation of power by circles which cut off segments directly from the lines $O A$ and $O B$.

In all that follows, we will give preference to another graphical method, still more simple, which will be explained in Chapter II.

Equation of the Synchronous Motor by the Method of Complex Variables. Finally, to complete the review of the different methods of study proposed or used for the representation and study of the phenomena, attention should be given to the equations which translate the preceding diagram by the method of complex variables, already applied to alternating current problems, in the exponential form, by Oberbeck, Cornu, Chaperon, etc., and in the linear form by Kennelly Steinmetz, Guilbert, etc. It is merely an application of the ordinary geometrical representation of imaginary quantities.

We will employ the notation of Steinmetz modified by M. Guilbert (La Lumiire Electrique, Vol. L, p. 451, and l'Eclairage Electrique, Vol. XIV, p. 69), i.e., by expressing the impedance as $(r+s i)$ instead of $(r-s i)$, the latter expression being scarcely logical, since the reactance acts in the same direction as a resistance to reduce the current. It thus becomes possible to retain the ordinary axes. ${ }^{3}$

(I) Let $O X, O Y$ (Fig. I4) represent two rectangular co-ordinate axes. Let $O A$ be a vector representing the sinusoidal function whose projections are $x$ and $y$. Geometrically, the vector $A$ is defined by the two projections, and analytically it is represented by a single imaginary value

$$
x+y j=(A \cos \theta)+(A \sin \theta) j,
$$

in which $j$ is the imaginary symbol $\sqrt{-\mathrm{I}}$.

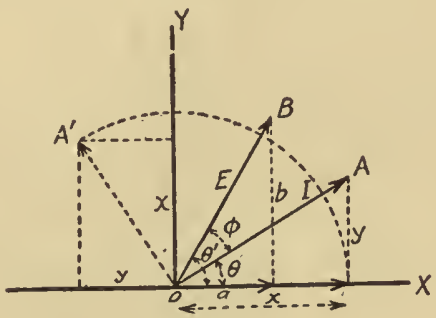

FiG. I4.

All the harmonic functions may be also represented by imaginary quantities.

${ }^{1}$ Electrical Transmission of Energy. London, Whittaker, I892.

${ }^{2}$ La Lunière Electrique, Vol. XLV, I892, p. 4I5, and Bulletin de la Société des Electriciens, 1893.

${ }^{3}$ Compare with C. P. Steinmetz, Theory of the Synchronous Motor (Transactions of the American Institute of Electrical Engineers, Oct. I7, I894) 
(2) These quantities are added or subtracted the same as real quantities: the resultant quantities indicate immediately, by their real and imaginary portions, the magnitude and the phase of the resultant vector.

(3) The multiplication of a complex quantity by a real quantity only changes the dimension of the vector, without changing its phase. On the contrary, the multiplication by an imaginary portion, such as $j b$, modifies not only the magnitude of the vector, which thereby becomes multiplied by $b$, but it also modifies its phase, which is then made to advance by the amount $\frac{\pi}{2}$. In fact, we have

$$
j(x+j y)=-y+j x
$$

i.e., the vector $O A$ is then replaced by the vector $O A^{\prime}$.

(4) From this, it follows that the E.M.F. absorbed by an impedance, $z$, composed of a resistance, $r$, and of a reactance, $s$, in series, through which passes a current

$$
i=x+j y,
$$

is obtained, in magnitude and phase, by forming the product

$$
z i=(r+j s)(x+j y) .
$$

(5) The work done by an E.M.F. $\overline{O B}$ having the imaginary value

$$
a+j b \text {, }
$$

and a current $\overline{O A}$ having the imaginary value

$$
x+j y,
$$

is easily obtained by decomposing it into the work of each of the components $a$ and $b$ of this E.M.F. The first component, $a$, does no work on the component $y$ of the current with which it is "in quadralure," but it is "in phase" with the component $x$; likewise $b$ is in quadrature with $x$ but in phase with $y$. The power required is therefore the sum of the products of the analogous coefficients of $E$ and of $I .^{1}$ We will have

$$
a x+b y \text {. }
$$

1 Use can also be made of other equivalent rules. A rule is given by M. Janet (Eclairage Electrique, I8. Dec., 1897, 1. 530) under the following form: To find the power, $j$ is changed into $-j$ in either the expression for the current strength 
Let us apply this notation to the generator and motor already considered. Let us direct the E.M.F. $E_{1}$ of the generator along the axis $O X . \quad E_{2}$, lagging in phase by the angle $\theta$, measured from the opposite direction, will have the form

$$
E_{2}(\cos \theta-j \sin \theta) .
$$

The imaginary impedance is

$$
R+\omega L j
$$

The resultant E.M.F. being

$$
E_{1}-\left(E_{2} \cos 0-j E_{2} \sin \theta\right),
$$

the current will be obtained by taking the quotient

$$
I=\frac{E_{1}-E_{2} \cos \theta+j E_{2} \sin \theta}{R+\omega L i}=\left(E_{1}-E_{2} \cos \theta+j E_{2} \sin \theta\right) \frac{R-\omega L j}{R^{2}+\omega^{2} L^{2}} .
$$

Separating the real and imaginary portions, we have

$$
I=\frac{I}{R^{2}+\omega^{2} L^{2}}\left\{\begin{array}{c}
R\left(E_{1}-E_{2} \cos \theta\right)+\omega L E_{2} \sin \theta \\
+j\left[R E_{2} \sin \theta-\omega L\left(E_{1}-E_{2} \cos \theta\right)\right]
\end{array}\right\} .
$$

This equation, when transformed into finite values, and taking $z$ to represent the impedance, gives

$Z^{2} I^{2}=\left[R\left(E_{1}-E_{2} \cos \theta\right)+\omega L E_{2} \sin \theta\right]^{2}+\left[R E_{2} \sin \theta-\omega L\left(E_{1}-E_{2} \cos \theta\right)\right]^{2}$

This equation defines the relation between $I, E_{1}, E_{2}$, and $\theta$ in a synchronous motor.

The mean power, $P_{1}$, is obtained by simply multiplying the real portion by $E_{1}$ thus:

$$
\begin{aligned}
P_{1} & =\frac{E_{1}}{R^{2}+\omega^{2} L^{2}}\left[R E_{1}-E_{2}(R \cos \theta-\omega L \sin \theta)\right] \\
& =\frac{E_{1}\left[E_{1} \cos \gamma-E_{2} \cos (\theta+\gamma)\right.}{\sqrt{R^{2}+\omega^{2} L^{2}}} .
\end{aligned}
$$

or the expression for the E.M.F. The product is calculated, and then only the real portion thereof is taken. M. Guilbert has also given the following rule (E. E., Io Mar., I900, p. 36r): Change the sign of the variable which is out of phase and the real portion gives the power, with its sign. 
As for $P_{2}$, it can, according to the rule already stated, be expressed as follows:

$$
\begin{aligned}
P_{2} & =\frac{\mathrm{I}}{R^{2}+\omega^{2} L^{2}}\left\{\begin{array}{c}
E_{2} \cos \theta\left[R\left(E_{1}-E_{2} \cos \theta\right)+\omega L E_{2} \sin \theta\right] \\
-E_{2} \sin \theta\left[R E_{2} \sin \theta-\omega L\left(E_{1}-E_{2} \cos \theta\right)\right]
\end{array}\right\} \\
& =\frac{E_{2}}{\sqrt{R^{2}+\omega^{2} L^{2}}}\left[E_{1} \cos (\gamma-\theta)-E_{2} \cos \gamma\right] .
\end{aligned}
$$

This expression could have been written directly, by symmetry.

Such is the method of imaginaries for establishing the fundamental equations. It will be seen that the calculations thereby made are more simple than with the analytical method of Hopkinson, because they give, immediately, the general solution, and do not require any integration. In reality, however, complex variables only constitute an artifice for writing down the results of the graphical method. In place of detailed reasonings which are rendered more precise by means of diagrams, they substitute algebraical operations, which are effected mechanically, without benefit in helping the mind to understand the physical phenomena. In all that follows we will therefore adopt the graphical method.

Excitation of Synchronous Motors. Synchronous motors may be excited in three ways:

(I) As in the case of alternators, it is possible to use the current produced by a small exciter-dynamo mounted on the same shaft. The machine is put in operation by one of the methods indicated later, or else by running the exciter-dynamo as a motor, by means of current from a storage battery. This method of excitation is advantageously used for large motors.

(2) To avoid the complication of the exciter-dynamo, especially for small motors, the machine is often made self-exciting by means of commutated currents, by sending through the field-circuit a portion of the current obtained from the mains. The oldest method of commutating this current consisted in using a simple shell-commutator by which the current was reversed at each change of polarity. Such was the Ganz commutator shown diagrammatically in Fig. ${ }_{5}$ for a six-pole motor. The inducing circuit connects with three segments $p, p^{\prime}, p^{\prime \prime}$ connected in parallel with one pole, and the other end of the circuit connects with three other segments $n, n^{\prime}, n^{\prime \prime}$, which are connected in parallel with the other pole. The alternating current to be commutated is 
brought by two double brushes $b b^{\prime}, b_{1} b^{\prime}$, which can be shifted to a suitable extent by means of the collar supporting them, the object being to reduce sparking to a minimum. The use of double brushes

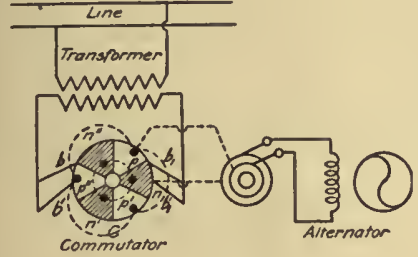

FIG. 15 .

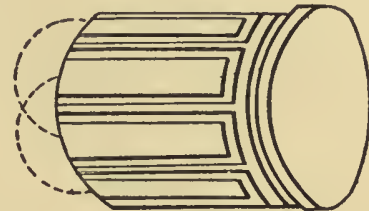

FIG. I6.

has for its object to reduce the sparking by short-circuiting the field windings at the time when the induced current is reversing; the extra current which is then produced in the exciting coils prolongs the current in the field-circuits and diminishes the undulations of the inducing magnetic flux. Nevertheless, these fluctuations are quite perceptible and interfere very much with the efficiency and the stability of the machine. These commutating arrangements can only be used for low voltages. For this reason, when the voltage at the terminals of the motor exceeds i io volts, the commutator is supplied by the secondary winding of a transformer which is connected in multiple with the

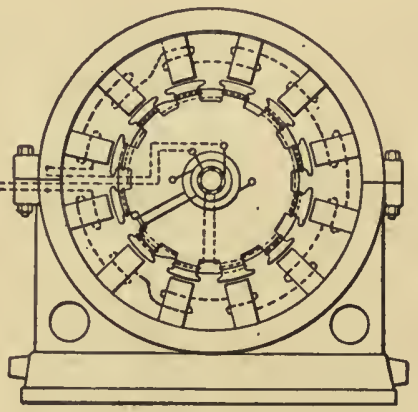

FIG. I7. mains or else by a special low-voltage circuit wound on the same armature, as in the case of the old Westinghouse alternators (Fig. I7).

Commutated currents can, theoretically, be used for excitation either in series or in shunt (even compound) as indicated in the diagram of Figs. I8 and I9. The advantages and disadvantages of these various systems are the following: 1

Series-excitation allows the use of large wires for the field-coils, and insures coicidence in phase between the currents of the inducing

1 A more detailed study of these "commutated" excitations will be found in an article on synchronous motors by the author, in La Lumière Electrique, 1892, Vol. III, pp. 465,466 , etc. 
and induced circuits during the operation of starting, which operation is thereby facilitated. It has, however, the great objection of making the induced E.M.F. vary with the load, whereas, according to thenry, the most advantageous E.M.F. is fixed $a$ priori, and should remain practically independent of the load. With series-excitation, strong wattless currents occur at very light and very heavy loads (which may, it is true, be reduced by shunting the field-coils, as shown in Fig. 19), and, besides, there is a lack of stability of operation at light loads

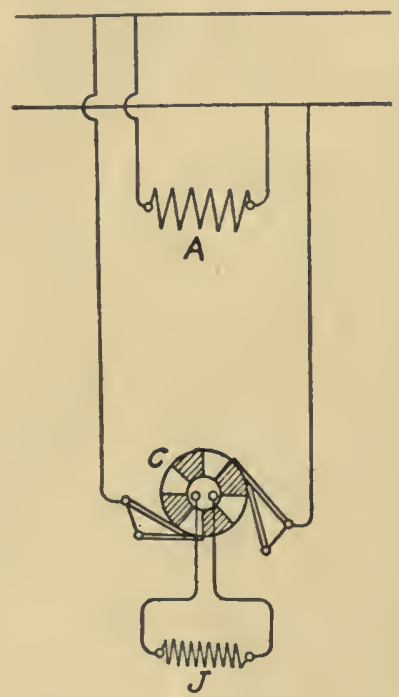

FIG. I8.

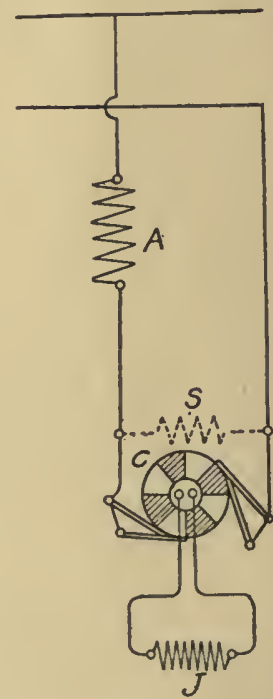

FIg. I9.

because the field is then too weak. Morevoer series-excitation causes very bad sparking at the brushes.

Shunt-excitation has the advantage of being constant and easily regulated; but it has another objection, that of necessitating change of position of the diameter of commutation with respect to the phase of the current to be commutated. With normal load, the position of the brushes can be easily regulated in such a way that the commutation may occur exactly when the alternating current to be commutated is passing through the zero-point; but if the load increases, the lagging of the armature behind the field increases, without being followed by the phase of the current to be commutated. The same thing occurs during any accidental oscillation of the speed of the armature (rotor). 
Now, as Fig. 20 shows, the mean strength of the commutated current is proportional to the algebraical sum of two areas, one of which $\left(S_{1}\right)$ is positive, and the other $\left(S_{2}\right)$ is negative. The former diminishes and the latter increases, when the lag increases, the effect being a rapid decrease in the excitation. If we let $t_{0}=$ time at which the commutation occurs; if $T=$ the period, and $2 \pi \frac{t_{0}}{T}=$ the lag . in question, the exciting ampere-turns will be given by the following equation:

$$
N i_{\text {av. }}=\frac{2 N}{T} \int_{t_{0}}^{\frac{T}{2}+t_{0}} i_{0} \sin \left(2 \pi \frac{t}{T}-B\right) d t=\frac{2}{\pi} N i_{0} \cos 2 \pi \frac{t_{0}}{T},
$$

i.e., they are reduced in the proportion indicated by the factor $\cos \frac{2 \pi I_{0}}{T}$, as compared with their value when the commutation occurs at the

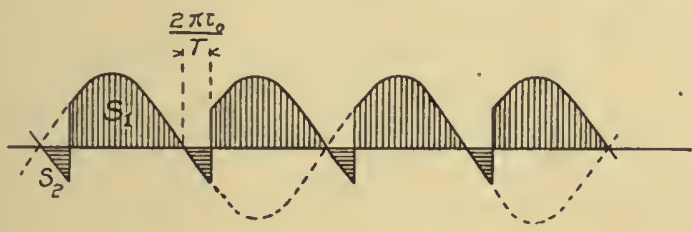

FIG 20.

proper moment of passing through zero (at which time the factor $\cos \frac{2 \pi t_{0}}{T}$ equals unity). It is necessary, therefore, to vary the position of the brushes with the load. This cannot be done, however, during the fluctuations of load, and it is conceivable that this decrease of excitation under the influence of the lag may then greatly reduce the stability of operation. It appears, therefore, that the method of excitation by commutated currents is a process open to criticism.

Advantage might be taken of the use of the transformer in Fig. $2 \mathrm{I}$, as the author showed in 1892 (Lumiere Electrique, loc. cit.), to compound the excitation in such a way as to cause the inducing flux to increase with the load, and thus produce an increase in stability. It would be sufficient, to this end, to wind on the same transformer a second primary coil with a large conductor through which the armature or rotor current passes.

(3) Another method of commutating the current, which is preferable to the preceding one, consists in combining with the armature- 
winding a continuous-current commutator from which the exciting current is collected by means of two brushes. Such is the system of the Fort Wayne Company in America, and of the Société l'Eclairage

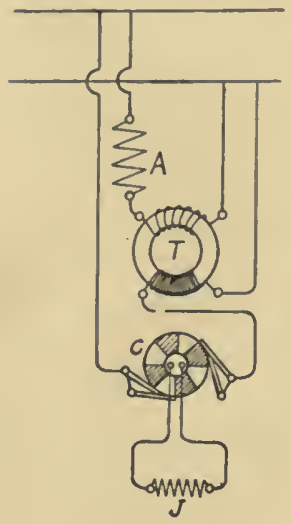

FIG. 21 . Electrique (Labour system) in France. The commutator need be only of small dimensions, proportional to the current which it delivers, unless it is also used in starting the motor. When the voltage of the armature is too high or when it is desirable to simplify the connections of the main circuit, it is sufficient to wind, on the armature, a small exciter winding connecting with a small commutator, which serves solely for the purpose of excitation.

This method of excitation, which is the simplest of all, unfortunately still presents several objections. It is applicable only to motors having continuous current windings and a rotating armature; it cannot be adopted in the case of high voltage machines or alternators having a stationary induced winding; it does not lend itself to automatic regulation of the inducing field; finally, (and this is the most serious objection), when there are wattless demagnetizing currents in the motor, from any cause, the E.M.F. at the brushes and the excitation are both weakened in consequence. Now, that is precisely what occurs when the motor experiences a reduction in speed in consequence of an overload. The stability of operation, which decreases with the excitation, will, therefore, be diminished when the load increases. The oscillations due to improper operation are therefore exaggerated by this reaction.

The self-excitation of synchronous motors must, therefore, be studied with care, and its application is limited to small motors. For larger machines the use of a separate exciter is the most satisfactory plan. 


\section{CHAPTER II}

\section{DETAILED STUDY OF OPERATION WITH NORMAL LOAD}

\section{PRINCIPLES OF THE ELEMENTARY THEORY}

\section{Notation. Let}

$R=$ the resistance of the total circuit of the motor (including the motor, the line, and the armature of the generator, in the case of an electric transmission system. (The armature resistance of the alternators is supposed to be increased from 50 to 100 per cent in such a way as to take into consideration the eddy currents produced by the armature in the pole-pieces);

$L=$ the mean inductance of the motor;

$l=$ that of the external circuit (including line and generator);

$T=$ the period; $\omega=\frac{2 \pi}{T}=$ the velocity of pulsation;

$X=$ the reactance of the total circuit, including the motor; $X=\omega(L+l)$;

$Z=$ the impedance of the total circuit, including the motor;

$$
Z=\sqrt{R^{2}+X^{2}}
$$

$E_{1}=$ the effective external E.M.F., i.e., the E.M.F. obtained from the line (or induced in the generator, in the case of an ordinary simple transmission system). This E.M.F. is supposed constant, unless otherwise stated, in all that follows;

$E_{2}=$ the effective internal E.M.F. induced in the synchronous motor, such as it would be measured on open circuit with the same excitation. ( $E_{2}$ should not be confounded with the resultant induced or effective E.M.F. produced by the resultant field of the field ampere-turns and of the armature-reaction. The latter will be designated, later, by the symbol $\varepsilon_{2}$.) $\varepsilon=$ the resultant E.M.F. of the two E.M.F's. $F_{1}$ and $E_{2}$;

$I=$ the current in the armature of the motor; $P_{1}=$ the electric power produced by the E.M.F. $E_{1}$; 
$P_{2}=$ the electric power generated in the armature by the current, and counted positively in the same direction as the mechanical power obtained from the motor;

$\theta=$ the phase-angle between the E.M.F's. $E_{1}$ and $E_{2}$;

$\phi=$ the phase-angle between the current, $I$, and the E.M.F. $E_{2}$, of the motor;

$\psi=$ the phase-angle between the current, $I$, and the external E.M.F., $E_{1}$. $\gamma=$ an auxiliary angle representing the phase-angle existing between the current and the resultant E.M.F., $\varepsilon$, which produces it in the circuit. It is defined by the known relation,

(I) $\tan \gamma=\frac{\omega(L+l)}{R}=X ; \quad$ whence $\left\{\begin{array}{l}\sin \gamma=\frac{Y}{Z}, \\ \cos \gamma=\frac{R}{Z} .\end{array}\right.$

$\delta=$ an analogous angle, obtained by neglecting the reactance of the motor. It is defined by the relation,

(2) $\tan \delta=\frac{\omega l}{R}$.

The standard diagram which has already been referred to is not easy to apply, partly because a complicated construction is necessary for each load to give the current-strength in magnitude and in phase, and partly because the vectors of the current-strength become too small, inasmuch as the E.M.F. may attain thousands of volts while the currents are of a few amperes only. The geometrical constructions deduced from this diagram by other authors are extremely complicated.

The author devised, some years ago, ${ }^{1}$ for the study of problems of this kind, a so-called "bipolar" diagram, which avoids these two objections and at the same time gives the theory an almost childlike simplicity, and renders graphical calculation sufficiently accurate.

Principle of Bipolar Diagrams. The principle of this method consists in taking different axes of reference and different scales for the currents and for the E.M.F's., instead of taking the same axis of reference and the same scale for both, as is usually done. The axes and scales may then be selected for the current in such manner that the

'Theorie des moteurs synchrones. Lahure, publ: 1895. L'Industrie Electrique, Feb., 1895 . 
current can be represented in magnitude and in phase by the same vector as the resultant E.M.F. of the circuit.

As regards the magnitude, since we have

$$
\varepsilon=Z I,
$$

it suffices to take, for the current $I$, a scale of amperes $Z$ times greater than the scale of volts 'used for the E.M.F. values.

As to the axis of reference, its choice depends on the axis selected for the E.M.F. values.

The bipolar diagram may therefore be established in two distinctly different manners, according to whether it be the vector of the generator E.M.F., $\mathrm{E}_{1}$ or that of the motor, $E_{2}$, which is taken as the fixed line.

We will indicate, successively, these two types of diagrams which, in many cases, do not serve exactly the same purpose, as will be seen later.

Bipolar Diagram of the First Kind. Motor-Vector $\mathrm{E}_{2}$ taken as Fixed Axis. Let us suppose the effective values $E_{1}$ and $E_{2}$ of the E.M.F's. taken with the signs which they have when one follows the circuit formed by the motor, the line, and the generator. It is known, by experience, that the E.M.F's. are very nearly in opposition. The two corresponding vectors can, therefore, be represented by two right lines $O A_{2}$ and $O A_{1}$, drawn in different directions (as indicated by the arrow-heads) and making, a very small angle, $\theta$, with each other (Fig. 22). (This angle indicates the phase-difference between the two E.M.F's., as measured at the terminals of the two machines when coupled in parallel.)

When under load, the generator tends to lead with respect to the motor. The vector $E_{1}$ will, therefore, turn in the positive direction which is conventional in mechanics, i.e., in the direction contrary to the hands of a watch.

The resultant E.M.F. $\varepsilon$, acting in the circuit, is represented by the vector $A_{1} A_{2}$, which is the resultant of $E_{1}$ and $E_{2}$. By projecting $A_{1} A_{2}$ on a right line, $A_{2} F$, which makes the angle $\gamma$ backward from $A_{1} A_{2}$, we obtain the vector $A_{2} F=R I$, representing the component that is in phase with the E.M.F. $E_{1}$. The angle $D A_{2} A_{1}$, therefore measures the phase-difference of the current with respect to the E.M.F. $E_{2}$ with. reversed sign, i.e., measured at the terminals, the same as the E.M.F. of the generator. In order to be able to take $A_{1} A_{2}$ as equal in 
magnitude and in phase to the vector of the current, it suffices, therefore, to attribute to it, as an axis of reference for phases, a right line $Y^{\prime} A_{2} Y$, which makes, with $O X$, the axis of reference for the E.M.F's., an angle equal to $r$, and having $A_{2} Y^{\prime}$ for its positive direction. We thus obtain the diagram Fig. 22.

This diagram is bipolar because the axis to which the E.M.F. $E_{2}$ is referred is the horizontal line $O X$, and the vector $E_{1}$ turns around $O$

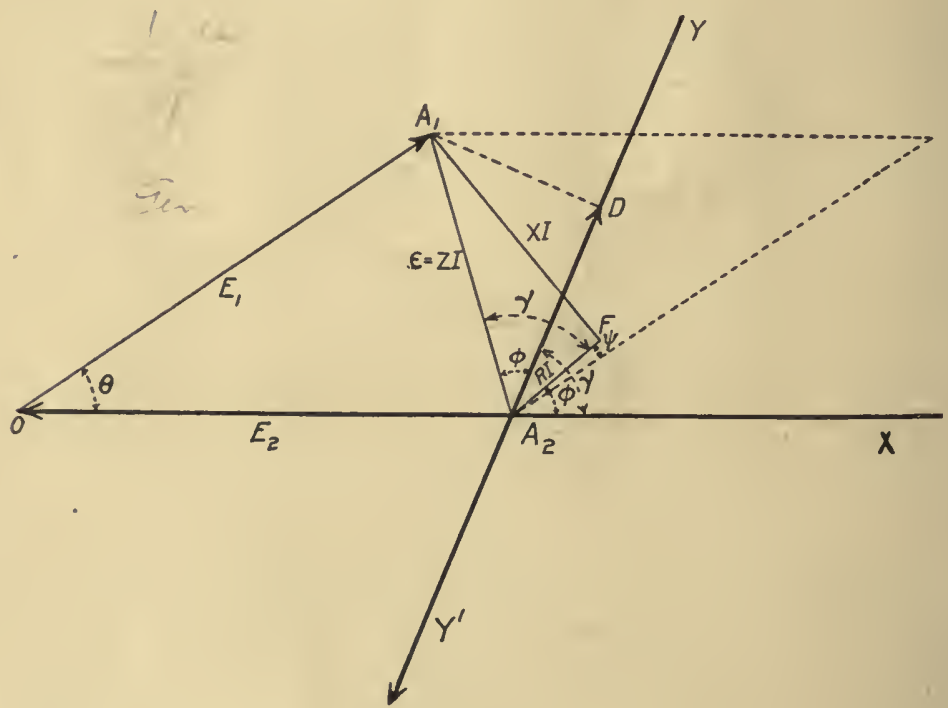

FIG. 22.

as a center; whereas the current vector $A_{1} A_{2}$ turns around $A_{2}$ and is referred to the axis $Y A_{2} Y^{\prime}$.

The diagram of Fig. 22 shows that the current is more or less directly opposed to the E.M.F. $E_{2}$. It can be decomposed into two components, one directly opposed, the other having a phase-difference of $\frac{\pi}{2}$. This decomposition is easily made on the diagram (Fig. 23) by projecting the vector $A_{2} A_{1}$ on the line $A_{2} Y$ and on a line $\left(A_{1} D\right)$ perpendicular thereto. We thus obtain:

(I) The component having the same phase as $E_{2}$ which, following the expression of Dolivo-Dobrowolsky, we will call the "watted" current,

$$
I_{w}=A_{2} D \text {. }
$$


DETAILED STUDY OF OPERATION WITH NORMAL LOAD 35

(2) The component having a phase-difference of $\frac{\pi}{2}$,

$$
I_{d}=A_{1} D
$$

The arrows indicate the direction of the vectors. Taking into consideration the direction of $Y Y^{\prime}$ (in the case of Fig. 22), it is seen that every wattless current $A_{1} D$, drawn to the left of the line of reference $A_{2} Y$, is a current which lags with respect to the E.M.F. $E_{2}$ but

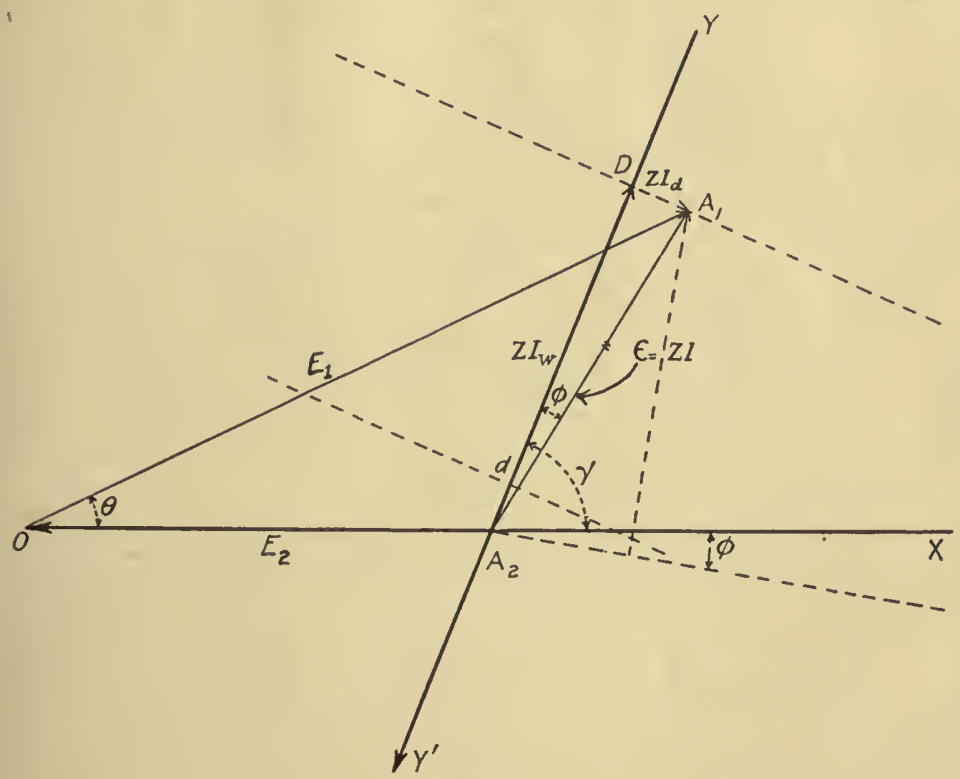

FIG. 23.

which leads with respect to the E.M.F. $E_{1}$. If the point $A_{1}$ should come to the right of $A_{2} Y_{1}$ (Fig. 23), the wattless current would lead with respect to $E_{2}$ but would lag with respect to $E_{1}$.

Applications of the Diagram of the First Kind. The diagram has for its object to enable the different working conditions of the motor to be grasped at a glance. It lends itself especially well to this when the motor E.M.F. $E_{2}$ is maintained constant, because only the point $A_{1}$ is then displaced, as the result of a variation either of the phase-angle $\theta$, or else of the generator E.M.F. $E_{1}$. In both cases the outputs are defined by the curve which $A_{1}$ describes and which we will call the "locus" of $A_{1}$. This locus is a circle when the excita- 
tion of the generator is also constant. Each position of the point $A_{1}$ defines the vector of the E.M.F. $E_{1}=O A_{1}$ and the vector of the current $Z I=A_{2} A_{1}$. Knowledge of any two whatever of the elements $E_{1}, \theta, I, \phi$ leads to that of the two others.

The diagram can be employed when $E_{2}$ varies, but the point $O$ then varies at the same time as $A_{1}$, so that the locus of $A_{1}$ no longer suffices to define the conditions of operation. There is then some advantage, if $E_{1}$ is constant, in having recourse to the second kind of diagram.

Line of Equal Power Occurring with Constant Excitation. The electric power of the motor, being equal to the product of the E.M.F. $E_{2}$ by the watted current $I_{w}$, will, when the excitation is constant, be proportional to the segment $A_{2} D$. When the motor-excitation remains constant, all outputs corresponding to equal watted currents are represented by points, $A_{1}$, situated on a single right line $A_{1} D$, perpendicular to the axis $A_{2} Y$. This right line $A_{1} D$ is, therefore, a line of constant power for the motor, whatever may be the E.M.F. of the generator, which may be variable. The same thing is true for all parallel right lines. It is possible to represent, on the diagram, a series of such right lines, each bearing the indication of the corresponding power $E_{2} I_{w}$ under constant excitation; and then, for each position of the point $A_{1}$, the corresponding power will be known.

The mechanical power is equal to the electric power less the losses due to eddy, currents and hysteresis. (The heat-losses are not included in the electric power $E_{2} I_{w}$ ). The effect of these losses is itself equivalent to a certain appreciable watted current, $j_{0}$, and to a wattless current which can be practically neglected. Therefore it will only be necessary to draw on $A_{2} Y$ a vector, $A_{2} d=Z j_{0}$, which represents the "lost" current; and the power that is really useful will be proportional to the segment $d D$. Although the loss under consideration varies slightly with the output, it can, without material error in constructing the diagram, be considered as constant for a given excitation, and as equal to the power necessary to operate the motor without load. The electric power supplied by the generator is deduced immediately from the electric power of the motor by adding the resistance-losses $\left(I^{2} R\right)$ in the circuit.

Lines of Equal Phase. Since the phase $\phi$ of the current is measured by the angle $A_{1} A_{2} D$, all the points representing outputs $A_{1}$, lccated on the same right line issuing from $A_{2}$, correspond to the same angle of lag $\phi$. Any line issuing from $A_{2}$ therefore constitutes a line of equal phase. 
Limit-Circle of Current. Let $I_{m}$ be the maximum value of the current which the armature can withstand. If, around $A_{2}$ as center, a circle be drawn with a radius equal to $I_{m}$ according to the scale of amperes (or equal to $Z I_{m}$ according to the scale of volts), this circle will constitute the boundary of a space having certain characteristics. All load-points inside that space correspond to loads which can be maintained indefinitely without the current, $I$, exceeding that limit. All load-points situated outside this space represent loads which cannot be maintained indefinitely. This limit-circle of current is, therefore, also a limit-circle of stability of operation, of the motor.

Algebraical Relations Deduced from the Diagram. To facilitate graphical calculation it is always useful to have the algebraical expressions for the variables. What we are interested in knowing is the current strength, its phase-angle, the power of the motor as a function of the E.M.F.'s $E_{2}$ and $E_{1}$, of the constants of the circuit, and of the angle of lag 0 , or conversely, any of these as a function of the power, etc.

The solution of the triangles represented in Fig. 23 gives immediately the relation sought. In the triangle $A_{1} D A_{2}$ we have

$$
\begin{aligned}
& I_{d}=I \sin \phi, \\
& I_{w}=I \cos \phi .
\end{aligned}
$$

Again, the projection $Z I$ is equal to the projection of the broken line $A_{1} D A_{2}$ :

$$
\begin{aligned}
Z I \cos (\gamma-\phi) & =Z I_{d} \cos \gamma+Z I_{d} \sin \gamma \quad \ldots \quad . \quad . \quad . \\
& =R I_{w}+X I_{d} .
\end{aligned}
$$

The triangle $O A_{2} A_{1}$ enables $E_{1}$ or $I$ to be expressed in terms of other quantities, thus:

$$
\begin{aligned}
& E_{1}^{2}=E_{2}^{2}+(Z I)^{2}+2 E_{2} Z I \cos (\gamma-\phi), \ldots . . . \\
& (I)^{2}=E_{1}^{2}+E_{2}^{2}-2 E_{2} E_{1} \cos 0 . \quad . \quad . \quad . \quad . \quad .
\end{aligned}
$$

Finally, if the triangle $O A_{2} A_{1}$ be projected on the line $A_{2} D$ and on a line $D A_{1}$ perpendicular thereto, we will have $I_{w}$ and $I_{d}$ as a function of $E_{1}, E_{2}$ and 0 :

$$
\begin{aligned}
& Z I_{w}=E_{1} \cos (\gamma-\theta)-E_{2} \cos \gamma \\
& Z I_{d}=E_{1} \sin (\gamma-\theta)-E_{2} \sin \gamma .
\end{aligned}
$$


The power of the motor and that of the generator will be easily obtained by noting that the phase-angle, which is $\phi$ with respect to $E_{1}$, becomes $\phi+0$. We will therefore have

$$
\begin{aligned}
& P_{2}=E_{2} I \cos \phi, \ldots . . \quad \text {. . . . . . } \\
& P_{1}=E_{1} I \cos \phi=E_{1} I \cos (\phi+\theta) ; \quad . \quad . \quad .
\end{aligned}
$$

or, replacing $I \cos \phi$ and $I \sin \phi$ by their values, $I_{w}$ and $I_{d}$, we have

$$
\begin{aligned}
& P_{2}=\frac{E_{2}}{Z}\left[E_{1} \cos (\gamma-\theta)-E_{2} \cos \gamma\right] \\
& P_{1}=\frac{E_{1}}{Z}\left\{\begin{array}{c}
\cos \theta\left[E_{1} \cos (\gamma-\theta)-E_{2} \cos \gamma\right] \\
-\sin \theta\left[E_{1} \sin (\gamma-\theta)-E_{2} \sin \gamma\right]
\end{array}\right\} ;
\end{aligned}
$$

which can also be written

$$
P_{1}=\frac{E_{1}}{Z}\left[E_{1} \cos \gamma-E_{2} \cos (0+\gamma)\right] .
$$

Numerical Example. Let us consider the case of a power-transmission to a distance of to kilometers by two Mordey alternators of 37 kilovolt-amperes (2000 volts and I8.5 amperes), having a frequency of roo cycles, the resistance being $3 \mathrm{ohms}$ and the reactance $43 \mathrm{ohms}^{1}$ Let us assume that the loss-allowance in the line is 200 volts at 20 amperes. The line will therefore have a resistance $r^{\prime}=$ ro ohms. If the conductors have a resistivity $(\rho)$ of 2 microhms per centimetercube, the sectional area of each conductor is

$$
\begin{aligned}
s=\frac{2 \rho l}{r^{\prime}}=\frac{2 \times 10^{-6} \times 20 \times 10^{5}}{\text { Io }} & =0.40 \mathrm{sq} . \mathrm{cm} . \\
& =0.062 \mathrm{sq} . \mathrm{in} .
\end{aligned}
$$

and the corresponding diameter will be $7.15 \mathrm{~mm}$. ( 0.28 inch). Let us assume that the distance between the wires is $60 \mathrm{~cm}$. (23.6 inches). The tables for line-inductances ${ }^{2}$ give, for the linear inductance, in millihenrys per kilometer, the following values:

$$
2(0.8 \mathrm{I} 88+0 . \mathrm{II} 73)=\mathrm{I} .872 \text {. }
$$

2 This reactance was determined directly from the characteristic curves given for these machines by Mr. Mordey. The value usually given for the inductance of this machine is ton low.

${ }^{2}$ See an article by the author in l'Eclairage Electrique, Oct.-Dec., 1894. 
DETAILED STUDY OF OPERATION WITH NORMAL LOAD 39

For a line of ro kilometers and a frequency of roo cycles, the reactance (assuming the capacity to be negligible) will therefore be

$$
k^{\prime}=\frac{\mathrm{ro} \times 628 \times \mathrm{r} .87^{2}}{\mathrm{r} 000}=\mathrm{I} \mathrm{I} .8 \mathrm{ohms},
$$

or approximately I 2 ohms.

Under these conditions, the constants of the circuit are, in round numbers, as follows:

$R=$ I 6 ohms, $K=98$ ohms; $Z=$ roo ohms; and we have

$$
\tan \gamma=\frac{98}{16}=6.12, \quad \cos \gamma=\frac{\mathrm{r} 6}{100}=0.16
$$

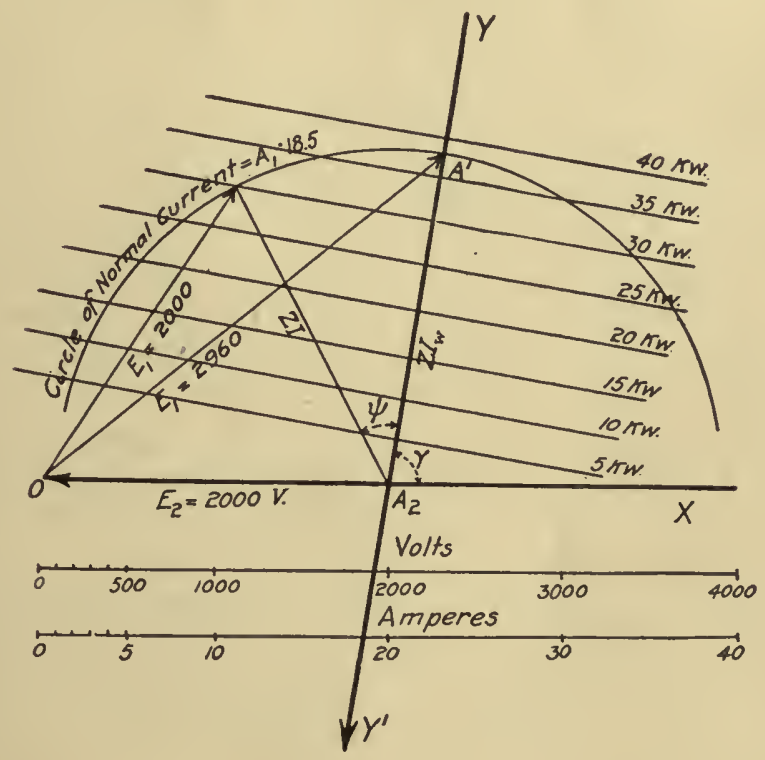

FIG. 24 .

The ratio of the two scales will be roo. Therefore a distance corresponding to 2000 volts on one scale will correspond to 20 amperes on the other scale.

Suppose, now, the E.M.F. of the motor to be $E_{2}=2000$ volts, and let it be represented by the stationary vector $A_{2} \mathrm{O}$ (Fig. 24) drawn on the axis of $O X$ in the negative direction. The right line $Y A_{2} Y^{\prime}$,making the angle $r$ with $O X$, backward, will be the origin of the vectors representing currents. The lines of equal electric power for the 
motor (not including resistance-losses in the armature) will be right lines perpendicular to $Y A_{2} Y^{\prime}$. In the diagram, several of these right lines have been drawn, corresponding to powers increasing each time by $5 \mathrm{k.w}$. Let us complete the diagram by describing, from $A_{2}$ as a center, with the radius $Z I=100 \times 18.5 A$, the circle of normal current. The point $A_{1}$, which depends on the load, can be displaced anywhere in the whole space inside the circle of normal current-value; but it cannot be displaced outside this space, except for a short time, owing to the heating of the machine. For each position of the load-point $A_{1}$, its distance from the point $O$, measured by the scale of volts, represents the corresponding E.M.F. of the generator, and the distance $A_{1} A_{2}$, divided by $Z$, represents the value of the corresponding current.

If we suppose the generator to be exactly like the motor, i.e., if its E.M.F. is 2000 volts, the diagram shows immediately that the maximum power practicably obtainable cannot exceed $30 \mathrm{k} . \mathrm{w}$. To attain 37.5 k.w. without exreeding the normal current, it would be necessary to raise the E.M.F. of the generator, $E_{1}$, to 2960 volts.

Diagram of the Second Kind. The Vector of the Generator

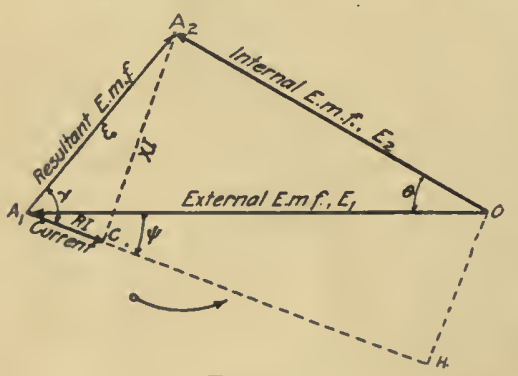

FiG. 25. E.M.F., $E_{1}$, as a Fixed Axis. Let the vectors corresponding to the generator and motor E.M.F.'s be represented by two right lines, $O A_{1}$ and $O A_{2}$, respectively (both being taken with the signs they present when the closed circuit is followed around). The angle $A_{1} O A_{2}$ (Fig. 25), which we will still call $\theta$, now measures the lag of $E_{2}$ behind $E_{1}$.

The current $I$ is equal to the resultant E.M.F. $A_{1} A_{2}$ divided by the impedance,

$$
I=\frac{\varepsilon}{Z}
$$

and it lags by the angle $\gamma$ behind $A_{1} A_{2}$. Its vector $\overline{A_{1} C}=R I$ is therefore the projection of $\overline{A_{1} A_{2}}$ over that angle, and the phase-difference $\psi$ between the current and the active E.M.F. $E_{1}$ is the angle $O A_{1} C$. In the case shown in the diagram, the current lags behind $A_{1} O$, i.e., behind the E.M.F. $E_{1}$ measured at the terminals of the generator.

The E.M.F. $\overline{O A_{1}}$ can be decomposed into two components, of 
which one, $\overline{A_{1} H}$, is in phase, and the other, $\bar{O} \bar{H}$, is in quadrature, with the current $I$. The effect of the motor is therefore equivalent to an apparent reactance, which is here positive,

$$
X_{\text {app. }}=\frac{\overline{O H}}{A_{2} C} X,
$$

combined with an apparent resistance

$$
R_{\text {app. }} \doteq \frac{\overline{A_{1}} \bar{H}}{A_{1} C} R \text {. }
$$

Finally, the electric power developed by each of the two E.M.F.'s $E_{1}$ and $E_{2}$ will be

and

$$
\left.\begin{array}{l}
P_{1}=E_{1} I \cos \left(\angle E_{1}, I\right)=\frac{\overline{A_{1} C}}{R} \times \overline{A_{1} H} \\
P_{2}=E_{2} I \cos \left(\angle E_{2}, I\right)=\frac{\overline{A_{1} C}}{R} \times \overline{C H}
\end{array}\right\} .
$$

where $\left(\angle E_{1}, I\right)$ and $\left(\angle E_{2}, I\right)$ mean the angle of lag between $E_{1}$ and $I$, and between $E_{2}$ and $I$, respectively.

The ohmic loss in the circuit $\left(I^{2} R\right)$ is equal to the difference $P_{1}-P_{2}$. Let us now transform the diagram, as above, in such manner that $A_{1} A_{2}$ shall represent the current, in magnitude and in phase. To obtain the magnitude, it will be sufficient to use, for measuring the amperes, a scale $Z$ times greater than the scale of volts, $Z$ being the numerical value of the impedance, expressed in ohms.

To obtain the lag of the current thus represented, with respect to the generator E.M.F. $E_{1}$, it will be sufficient to draw (Fig. 26). a stationary right line $\overline{A_{1} N}$ making the angle $\gamma$ in advance of $O A_{1}$. It will be seen immediately, on comparing Figs. 25 and 26 , that the angle $N A_{1} A_{2}$

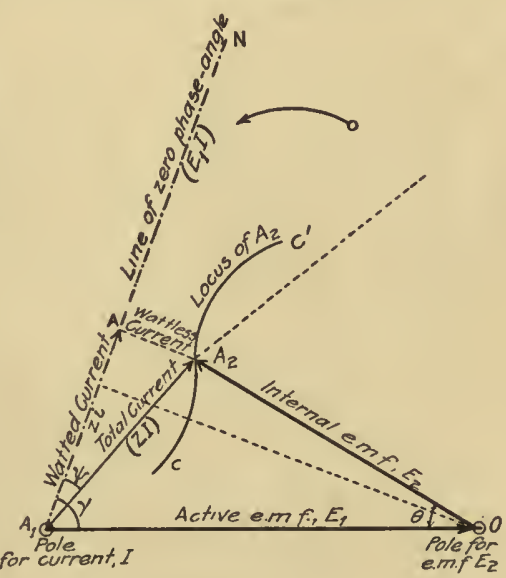

Fic. 26. formed by the vector $A_{1} A_{2}$, with the line $N A_{1}$ is equal to the angle $O A_{1} C$ previously referred to, and, consequently, represents the apparent phase-angle $\psi$. 
[To make the diagram complete we should draw two similar diagrams, turned in different directions, one corresponding to the volts, the other to the amperes, each with its own special scale. However, this would be of no advantage, and we will, therefore, retain a single diagram with the two scales, remembering that the origin of phases is $O A_{1}$ for the E.M.F.'s, and $A_{1} N$ for the currents. We shall indicate, on that diagram, the expressions for the segments of lines, as if they were all E.M.F.'s; and it will be sufficient to divide them all by $Z$ to have their expressions for currents.

For example, the segments $\overline{O A}_{1}$ and $\overline{O A}_{2}$ will represent, respectively, by the scale of amperes, the two currents

$$
\left.\begin{array}{l}
J_{1}=\frac{E_{1}}{Z} \\
J_{2}=\frac{E_{2}}{Z}
\end{array}\right\},
$$

which each one of the alternators would produce, respectively, in the circuit, if the other were stopped.]

The line $A_{1} N$ will therefore be the origin of phases for the current with respect to the E.M.F. $E_{1}$.

If we project $A_{1} A_{2}$, on the line $A_{1} N$ and on the line $A A_{2}$ perpendicular thereto, we will obtain, by the scale of amperes, two current-components:

(a) The watted current $i=\overline{A_{1} A}$, or the current which is in phase with the E.M.F.

(b) The wattless current $j=\overline{A_{2} A}$, cr the current which is in quadrature with the E.M.F.1

The phases of the two currents are both referred to $A_{1} N$ as origin.

The work of Dobrowolsky has shown that this decomposition of the current is very interesting from a practical standpoint; it is more so, in general, than the decomposition of the E.M.F. already indicated (page 4I).

The power furnished by the active E.M.F. may be deduced from the watted current, thus:

$$
P_{1}=E_{1} \times i \text {. }
$$

The power actually utilized may be deduced from this, in the following manner:

$$
P_{2}=P_{1}-r_{2} I^{2}
$$

in which $r_{2}=$ the armature-resistance of the motor.

1 'These terms, "watted" and "wattless," are adopted here to conform to the international language of manufacturers, although they are objectionable. 
Power-Values as Function of the Lag-Angle $\theta$. To express the power-values $P_{1}$ and $P_{2}$ in terms of the angle $\theta$, it is only necessary to substitute, in the two equations ( 13$)$, other values for $I \cos \left(\angle E_{1}, I\right)$ and for $I \cos \left(\angle E_{2}, I\right)$.

$1 \cos \left(\angle E_{1}, I\right)$ is nothing more than the watted current $i$ for the case represented in Fig. 23.

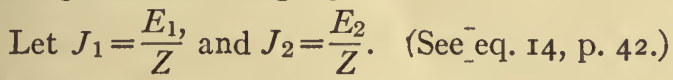

The diagram gives, readily,

$$
i=I \cos \left(\angle E_{1}, I\right)=J_{1} \cos \gamma-J_{2} \cos (\theta+\gamma) .
$$

The current $I \cos \left(\angle E_{2}, I\right)$ will be obtained by analogy, by projecting the diagram of Fig. 26 upon a right line making with $O A_{2}$ an angle that is again equal to the angle $\gamma$. From this we obtain the absolute value

$$
I \cos \left(\angle E_{2}, I\right)=J_{1} \cos (\gamma-\theta)-J_{2} \cos \gamma . . .
$$

Replacing $J_{1}, J_{2}$ by their values, $\frac{E_{1}}{Z}, \frac{E_{2}}{Z}$, we will therefore have

$$
\begin{aligned}
& P_{1}=\frac{E_{1}}{Z}\left[E_{1} \cos \gamma-E_{2} \cos (\theta-\gamma)\right] . \\
& P_{2}=\frac{E_{2}}{Z}\left[E_{2} \cos (\gamma-\theta)-E_{2} \cos \gamma\right] .
\end{aligned}
$$

These are expressions wherein only two variables, $E_{2}$ and $\theta$, enter. The utility of these expressions will be shown later.

Use of this Diagram for the Study of Different Loads. These loads cannot be determined without imposing some condition which makes the variables (i.e. E.M.F. current, power, lag, etc., ) vary according to some definite law. In that case, to each value of the lag there will correspond a single (or several) quite definite values of the E.M.F. $E_{2}$, and vice versa. Consequently, on the diagram, the point $A_{2}$, instead of being able to range over the whole surface, can be displaced only on a single curve such as $C C^{\prime}$, for example (Fig. 26), whose equation, in polar co-ordinates, with respect to the point $O$ as a pole, is nothing more than the relation imposed between $E_{2}$ and $\theta$.

For each point of this curve, which may be called the locus of $A_{2}$, there correspond two radial vectors issuing from two fixed poles.

One of these vectors, $\mathrm{OA}_{2}$, is drawn from the point $O$ as a pole; and it indicates the E.M.F. $E_{2}$, in magnitude and in phase (starting from $O A_{1}$ ); the other, $A_{1} A_{2}$, is drawn from the point $A_{1}$ as a pole; 
and it indicates the corresponding value of the current, in magnitude and in phase (starting from $A_{1} N$ ).

Knowledge of any two of the elements $E_{2}, 0, I$, enables all the others to be known, and also the lag $\psi$ and the reactive and active currents.

When $E_{1}$ is variable, this diagram becomes inconvenient, and preference should be given to the diagram of the first kind.

Curves of Constant Electric Power of the Motor when the Generator has Constant Excitation. These curves, which indicate

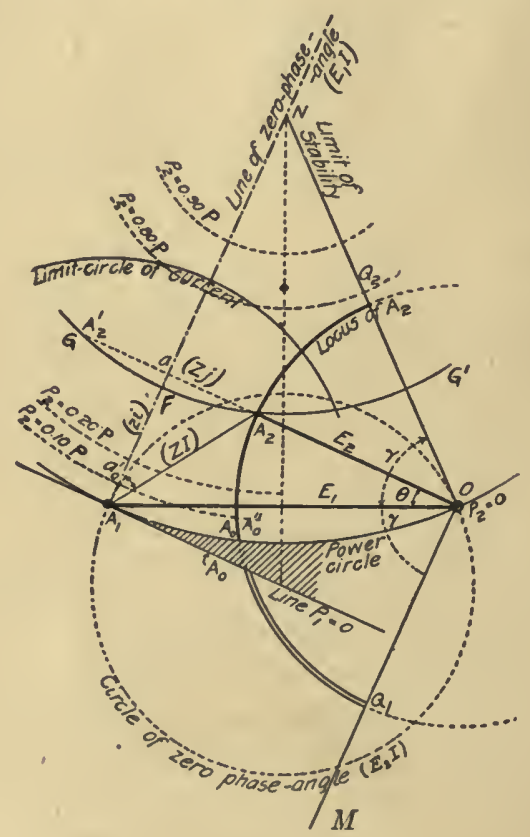

FIG. 27.

the power developed by the motor for each position of $A_{2}$, in the diagram, are represented by Eq. ( 18 ), in polar co-ordinates referred to the pole $O$ (Fig. 27) ( $P_{2}$ being supposed constant), and they now become circles instead of straight lines. Let us take two rectangular axes passing through $O$, the axis of $x$ being directed along the line $O N$, which makes with $O A_{1}$ the angle $\gamma$. We can then write

$$
\left\{\begin{array}{l}
E_{2} \cos (\gamma-0)=x, \\
E_{2}{ }^{2}=x^{2}+y^{2}
\end{array}\right.
$$


By substitution, we have

or

$$
\begin{gathered}
x^{2}+y^{2}-\frac{E_{1} x}{\cos \gamma}=-\frac{P_{2} R}{\cos ^{2} \gamma}, \\
\left(x-\frac{E_{1}}{2 \cos \gamma}\right)^{2}+y^{2}=\frac{\frac{1}{4} E_{1}^{2}-P_{2} R}{\cos ^{2} \gamma},
\end{gathered}
$$

from which it can be seen that the curve is a circle whose center has the following co-ordinates:

$$
x=\frac{E_{1}}{2 \cos \gamma} ; \quad y=0 .
$$

Each circle has for its center the point of intersection, $N$, of the right lines $O N$ and $O A_{1}$, both making the angle $\gamma$ with $O A_{1}$; and the radius of this circle is

$$
\rho=\frac{\mathrm{I}}{\cos \gamma} \sqrt{\left(\frac{E_{1}}{2}\right)^{2}-R P_{2}} \ldots \ldots . . .
$$

This result is also easily obtained by geometry.

As for the lines of constant electric-power, $P_{1}$ (applied at the terminals), they are evidently straight lines perpendicular to $A_{1} N$ (none of which has been drawn on the diagram except the line $P_{1}=0$ ); because they are defined by the simple condition that the current which is active with respect to $E_{1}$, .(i.e., $i$ ), is constant.

The expression (19) can be calculated algebraically, for various values of $P_{2}$, or it can even be determined graphically by means of rectangular triangles. It is convenient to express the power $P_{2}$ as a function of the maximum power which can be obtained by making $E_{2}$ vary, $E_{1}$ being supposed to remain constant. As will be seen later this power, which will be designated by the letter $\mathbf{P}$, has the following value:

$$
\mathbf{P}=\frac{E_{1}^{2}}{4 R}
$$

It is easy to construct a series of equipotential lines corresponding to the given values of $P_{2}$, and thus to predetermine the variations of power resulting from the displacements of the point $A_{2}$.

The radius $\rho$ can then be written,

$$
\rho=\frac{E_{1}}{2 \cos \gamma} \sqrt{\mathrm{I}-\frac{P_{2}}{\mathbf{P}}}
$$


It is therefore sufficient to divide the segment $\overline{A_{1} N}$ in the proportion of $\sqrt{\mathrm{I}-\frac{P_{2}}{\mathrm{P}}}$ to obtain the power-lines shown in Fig. 27 .

If, for example, we take the series of values,"

$\frac{P_{2}}{\mathbf{P}}=0.10,0.20,0.30,0.40,0.50,0.60,0.70,0.80,0.90,1.00$,

we will find

$\frac{\rho}{A_{1} N}=0.949,0.894,0.837,0.774,0.707,0.632,0.548,0.447,0.316$

In practice, these lines should be graduated in kilowatts, in the usual way.

It is often unnecessary to draw the lines for the high powers, since the power $\mathbf{P}$ is much greater than that which the armature can with. stand without dangerous heating, in continuous operation.

Since $\cos \gamma$ is very small (usually $<0.10$ ), in ordinary practical cases, the center $N$ of the circles will often be outside the diagram, but it will be always easy to draw the circles by points, from their equation in rectangular co-ordinates.

Moreover, in that case, the circles are of very large radius, and are quite sufficiently determined by two tangents. Now for each circle, such as $G F G^{\prime}$, the directions of the two tangents at $F$ and $G^{\prime}$ are known, because they are, respectively, perpendicular to $A_{1} N$ and $O N$. Again, the position of the points $F$ and $G^{\prime}$ corresponding to a power $P_{2}$, is given by the obvious equation :

$$
\overline{A_{1} F}=\overline{O G^{\prime}}=\frac{E_{1}}{2 \cos \gamma}-\rho=\frac{E_{1}}{2 \cos \gamma}\left(I-\sqrt{I-\frac{P_{2}}{\mathbf{P}}}\right) .
$$

It is very easy, therefore, to construct these two tangents and then to draw approximately the circular arc which they determine.

It will be noted that the " $P_{2}$ " power-values, in Fig. 27 , are positive above the point $A_{1}$, and negative below it. This shows that the alternator will operate as a motor in a certain portion of the plane, contained inside the power-circle $P_{2}=0$, and that it will operate as a generator beyond that circle. In the cross-hatched space comprised between the lines $P_{2}=0$ and $P_{1}=0$ it cannot operate either in one way or in the other, because the current which would then pass through the machine would cause a loss of energy in the armature greater than the available. power. 
Current-limit Circle. It is known that the armature cannot indefinitely withstand a current exceeding a certain limiting value $I_{m}$. If we draw, around the pole $A_{1}$ as a center, a circle of radius precisely equal to $I_{m}$ (by the scale of amperes), all the points $A_{2}$ situated outside that circle will give values $I=\overline{A_{1}} \overline{A_{2}}>I_{m}$; whereas, inside that circle the value of $I$ will be $\left\langle I_{m}\right.$. The circle drawn is therefore a limit beyond which the load-point must not go, for continuous operation.

Lines of Equal Phase. In the same way that lines of equal power have been drawn, lines of equal current-phase can be easily drawn, such that, at the loads represented by their various points, the phaseangle between the E.M.F. and the current will always be the same.

If the phase of the current $I$ is measured from the E.M.F. $E_{1}$, these lines are evidently straight lines issuing from the point $A_{1}$. The right line $A_{1} N$ corresponds to "zero" phase-angle. All the loads to the right thereof correspond to a lag in phase, and all the loads to the left correspond to a lead in phase. (Fig. 27.)

To obtain lines corresponding to currents of equal phase with respect to the E.M.F. $E_{2}$, we note that all the points for any current value whatever, among these lines, should present a constant angle $O A_{2} A_{1}$. These lines are therefore circles having $O A_{1}$ as a chord. If, for example, we wish the line of phase corresponding to the lag $\alpha$ between $E_{2}$ and $I$, we construct, on $O A_{1}$ as a chord, a circle admitting of the angle $\alpha-\gamma$ (since the true phase of the current lags by the angle $\gamma$ with respect to the line $\overline{\left.A_{1} A_{2}\right)}$.

Only the circle corresponding to an angle $\alpha=\pi$ has been drawn, in the example represented in the diagram. This circle represents the exact opposition in phase between the current and the internal E.M.F. $E_{2}$. It is easily seen that this circle is tangent to the two lines $O N$ and $A_{1} N$.

For all loads corresponding to points situated inside this circle, the phase is $>\pi$, outside the circle, on the contrary, it is $<\pi$.

[It will be noted, in passing, that the circle of zero power $P_{2}=0$, passing through the point $A_{1}$ is nothing more than the circle of phase $\frac{\pi}{2}$, because the power is zero every time that the current is in quadrature with the E.M.F.]

Numerical Example. Let us return to the example of power-transmission by means of two Mordey alternators, for which the numerical data have already been given (page 38), and let us apply the second diagram to this case. 
The maximum theoretical power is

$$
P=\frac{I}{16}\left(\frac{2000}{2}\right)^{2}=62,500 \text { watts. }
$$

The radii of the power-circles are given by eq. (2I); whence we have

$$
\rho=6250 \sqrt{1-16 \frac{P_{2}}{10^{6}}}
$$

in which $P_{2}$ is expressed in watts, $\rho$ being measured by the scale of volts. In this manner we find the radii for the circles of 5 I0 $20 \quad 30 \quad 40$ and $50 \mathrm{k} . \mathrm{w}$. to be $6000,5700, \quad 5 \mathrm{I} 30,4520,3750$, and 2800 volts:

These simple calculations give all the elements necessary for the diagram in Fig. 28. In this case the current-circle corresponding to

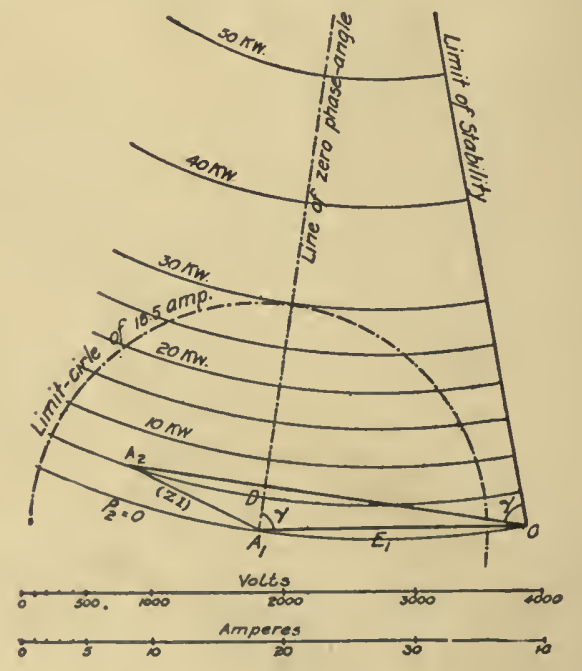

FIG. 28.

the normal current of 18.5 amperes has also been drawn, in addition, to show the limit of loads. Let $A_{2}$ be any position whatever of the load-point, within this circle; it is only necessary to measure the various lines, according to the proper scales, to see that the corresponding voltage $E_{2}$ is 3000 volts, the current strength is II amperes, the reactive current (leading) is 10.5 amperes, and the power is $5 \mathrm{k.w}$. The 
minimum current corresponding to the same power would be $O D=$ 2.8 amperes, approximately.

II. OPERATION OF A MOTOR WITH CONSTANT EXCITATION, SUPPLIED AT CONSTANT E.M.F.

The two diagrams lend themselves equally well to the discussion of this case, which is usually the most frequent one in practice. In the first diagram (Fig. 29) the E.M.F. vector of the motor will remain stationary and that of the generator will turn round the pole $O$, when

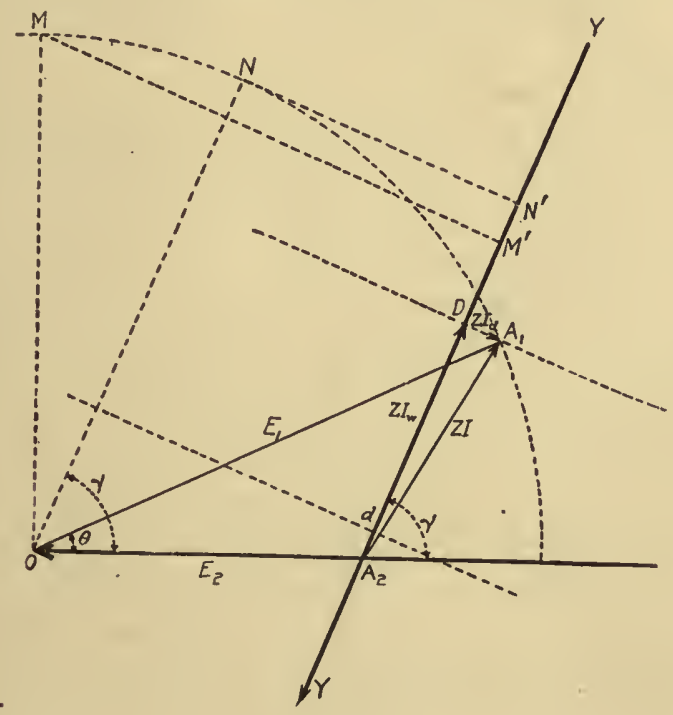

FIG. 29.

the load varies. The extremity $A_{1}$ describes a circle having $O$ for its center and $E_{1}$ for its radius. If the lines of equal load have been drawn, their points of intersection with the circle will define completely the conditions (currents and lags) for each load. The useful active currents will be obtained by subtracting the loss-current $\frac{A_{2} d}{Z}$ from the active currents themselves.

It will be noted that the power is zero when $A_{1}$ is on the power line passing through $d$. If, in consequence of overload, the motor- 
speed diminishes, $\theta$ increases and the power developed also increases. The condition is therefore stable for low powers.

But the power-output is limited. Its maximum corresponds to the power-line drawn tangentially to the circle. The corresponding output, $N$, is the limit of possible outputs, because the effect of an overload, by increasing further the lag $\theta$, would be to bring the vector $O A_{1}$ into a position such as $O M$, where the power-output would be diminished, and, consequently, the motor would stop.

The line $O N$, drawn through $O$ parallel to $A_{2} Y$, therefore defines theoretically the limiting line of stability; and the limiting lag between the E.M.F.'s. is equal to $\gamma$. The maximum electric power applied to the motor is equal to the product of the E.M.F. $E_{2}$ by the corresponding active current $\frac{A_{2} N^{\prime}}{Z}$.

Likewise, on the second diagram (Fig. 27), if the excitation is supposed to be constant and if the load is made to vary, the end of the E.M.F. vector $E_{2}$ describes a circle around $O$ as a center. Its point of intersection, $A_{0}$, with the circle of zero-power $\left(P_{2}=0\right)$, gives, theoretically, the condition of operation without load; but, practically, the powver necessary to overcome friction and other losses not being equal to zero, the unloaded condition will correspond to a higher point, such as $A^{\prime \prime}{ }_{0}$, situated on the power-circle $P_{2}=0$.10 $\mathrm{P}$.

When the load applied to the motor-shaft increases, the point of output $A_{2}$ is displaced on its circle in the direction of increasing poweroutputs, i.e., toward the top, and the angle of $\operatorname{lag} \theta$ and the current $I$ increase correspondingly.

The power can increase up to the point $Q_{2}$, where the characteristic is tangent to a power-circle (Fig. 27); but it could only decrease if the angle of lag were to be further increased. . The direction $O N$ therefore marks the theoretical limit of stability of operation. When the angle of lag is increased further the motor falls out of step and comes to a stop.

It may be seen in the same manner that the alternator operates as a generator below the point $A^{\prime}{ }_{0}$, and that the necessity of stability of operation limits the angle of lag to the direction $O M$, which is perpendicular to the right lines of equal power $P_{1}$, and symmetrical with $O N$, with respect to $O A_{1}$.

Maximum Power. The maximum power $P_{2}$, corresponding to the limit of stability just determined, can be easily calculated by making $\theta=r$ in the general power equation (18). 
From this we get

$$
\left.\begin{array}{rl}
P_{2 \max .} & =E_{2} \frac{E_{1}-E_{2} \cos \gamma}{Z} \\
& =E_{2} \frac{\cos \gamma\left(E_{1}-E_{2} \cos \gamma\right)}{R} \\
& =\frac{E_{2}}{Z}\left(E_{1}-E_{2} \frac{R}{Z}\right) \\
& =\frac{E_{2}}{R} \frac{R}{Z}\left(E_{1}-E_{2} \frac{R}{Z}\right)
\end{array}\right\}
$$

The power-value will be higher in proportion as the impedance $Z$ and the resistance $R$ are smaller (provided that $\cos \gamma<\frac{\mathrm{I}}{2} \frac{E_{1}}{E_{2}}$ ).

The formula (22), can also be written directly from the diagram (Fig. 29) corresponding to the load which is at the limit of stability, because, we have, from this diagram,

$$
Z I_{w}=E_{1}-E_{2} \cos \gamma,
$$

and also

$$
P_{2}=E_{2} I_{w} \text {. }
$$

In well-constructed modern motors (having low resistance and low impedance), there is usually no tendency of the machine to fall out of step from gradual overloading, when it is supplied from a constant potential circuit, because, as Fig. 28 shows clearly, the current becomes excessive before the power-outputs approach the limit of stability just indicated. For example, the Labour synchronous motors, which can be cited as excellent existing examples of this class of apparatus, are only limited, in regard to load, by the heating of the armature, and not by the tendency to fall out of synchronism. It is the circle of maximum current, drawn as we have already seen, which limits longcontinued loads.

But loss of synchronism may result from anything which can increase the impedance and diminish the reactance-factor, and, consequently the tendency to fall out of step is increased by the resistance of the line in long-distance transmission.

It is therefore necessary to be able to foresee, under certain circumstances, whether the operation will be stable or not.

Means of Determining the Practical Stability of Synchronous Motors. In practice, owing to oscillations of speed, the load is restricted to a value which is less than the maximum power just determined. It can be conceded, in all cases, that, during these speed-oscillations, 
the excitation of the motor (even if it is compounded) has not time to vary. It can, therefore, always be assumed that the motor has a constant induced E.M.F., and the results of experiments made with such motors can always be used as the starting point.

On this basis, the stability of operation can be calculated in a sufficiently precise manner by simply comparing the effective load of the motor at the maximum power, $P_{m}$, which it might develop with

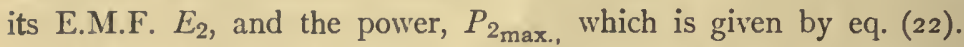

[This value can be determined experimentally by the method set forth in the author's pamphlet on the "Coupling of Alternators", Paris, I 894 , p. 4.]

Experience shows that $i$ is possible to have stable operation so long as the power $P_{2}$ does not exceed about two-thirds of $P_{m}$, when the apparatus driven by the motor constitutes a very constant load, or about the half of $P_{m}$, when the load is somewhat irregular. [It is assumed, of course, that the apparatus can withstand the corresponding current without overheating.]

These figures have been verified by various experiments and they are in accordance with the results published by other experimenters. On analyzing the power transmission at Cassel (see La Lumiere Electrique, Vol. XLV, p. 6I6) where the motors furnish power for charging batteries, we find that the ratio of practical to theoretical maximum power is as high as $\frac{P_{2}}{P_{m}}=\frac{4}{5}$, but this is exceptional. It is safer to assume $\frac{P_{2}}{P_{m}}=\frac{\text { I }}{2}$.

Variations of Stability with Operating Conditions. It is very evident that, all things being equal, the stability will decrease with the load. To see how it varies with the conditions of the circuit and of the motor-excitation, when the power $P_{2}$ and the voltage $E_{1}$ are constant, it is only necessary to study the variations of the power $P_{m}$, by means of equations (22). With equal resistance it presents a maximum of maximums

$$
\mathbf{P}=\frac{E_{1}^{2}}{4 R}
$$

whenever $E_{1}, E_{2}, Z$, and $\gamma$ satisfy the condition

$$
E_{2}=\frac{E_{1}}{2 \cos \gamma}=\frac{E_{1} Z}{2 R}
$$


(I) Suppose a given motor-load, and suppose the E.M.F.'s. $E_{1}$ and $E_{2}$ to be nearly equal. The stability will first increase with the reactance until we have

$$
\frac{Z}{R}=\frac{2 E_{2}}{E_{1}}
$$

i.e., until $Z$ approaches $2 R$; and it will then decrease if $\frac{Z}{R}$ is greater. Inasmuch as $\frac{Z}{R}$ is always greater than 2 in ordinary alternators, any increase of inductance is objectionable in a motor which is connected directly to the source of current. It could only be useful if the motor was connected to the generator by a very long line of exceptionally high resistance.

(2) Suppose a given motor-load and suppose the external E.M.F. $E_{1}$ to be constant. The stability of operation will always vary inversely with $R$; but the result will depend upon whether $\cos \gamma$ be greater or smaller than $\frac{1}{2}$, i.e., whether the reactance-factor $\left(\tan \gamma=\frac{X}{R}\right)$ will be higher or lower than $\sqrt{3}$. When $\cos \gamma>\frac{1}{2}$ an increase of the internal E.M.F. $E_{2}$ until it exceeds the external voltage $E_{1}$ will cause an increase of stability, whereas, when $\cos \gamma<\frac{1}{2}$ the increase will cause a reduction of stability. This observation explains someapparent contradictions of experience. When a motor is connected on a constant potential line or on a line of low resistance, it is more difficult to make it fall out of step when it is over-excited. If, on the other hand, the same motor is used for powertransmission over a line of high resistance, it will be observed that increasing the excitation will also increase its tendency to fall out of step.

In practice, the first case is the more frequent, and it is advisable, in order to improve the stability, to increase the excitation with the load either by hand or automatically.

At the same time, it is seen that, in the most frequent practical cases, it is necessary, in order to have good synchronous motors, to diminish both the resistance and the reactance of the armature. This means that the field must be powerful and the armature-reaction must be low.

If we were to make $Z$ constant by hypothesis, we would be led to reduce as much as possible the resistance and to increase the reactance; but this case does not occur in practice.

Numerical Example. Let us take, for example, two Mordey alternators of 37.5 kilowatts electrical output ${ }^{1}$ coupled for electrical trans-

${ }^{1}$ The power available at the shaft must be reduced in proportion to the efficiency (exclusive of the excitation-energy). 
mission, and let $E_{1}=2200$ volts be the generator E.M.F. The internal resistance of each machine is $3 \mathrm{ohms}$ and its reactance at 100 cycles is $4.3 \mathrm{ohms}$, as previously stated.

(r) Let us first suppose the line to have no resistance and no reactance. The total circuit will have the following constants-resistance $6 \mathrm{ohms}$, reactance $86 \mathrm{ohms}$ and impedance $86.20 \mathrm{ohms}$.

The maximum power

$$
P=\frac{(2200)^{2}}{4 \times 6}=201,660 \text { watts, }
$$

could only be obtained by giving to the motor E.M.F. the value

$$
E_{2}=\frac{\mathrm{I} 100}{0.0695}=\mathrm{I}_{5}, 800 \text { volts, }
$$

and it could only be obtained with a current

$$
I=\frac{2200}{2 \times 6}=\mathrm{I} 8_{3} \text { amperes. }
$$

It is therefore unattainable from all points of view.

If we limit the E.M.F.'s to $E_{2}=E_{1}=2200$ volts, the possible maximum will be

$$
\left(P_{2}\right)_{\max }=\frac{(2200)^{2}}{86.20}(\mathrm{I}-0.0695)=52,000 \text { watts; }
$$

i.e. about $3 / 2$ times the normal output. The stability will therefore be sufficient provided the machines are not subjected to variations of load.

Every increase in voltage will here produce an increase of $\left(P_{2}\right)_{\max }$. If, for example, we take $E_{2}=3000$ volts, $69 \mathrm{k.w}$. instead of $5^{2}$ is found to be the maximum output possible without causing the machine to fall out of step. The stability is then excellent at the normal load of $37.5 \mathrm{k.w}$. If, on the contrary, the voltage is reduced, so high a load could no longer be attained.

(2) Suppose now the resistance of a line to be interposed between the two alternators. Stability will svidently become impossible as soon as the maximum power $\mathrm{P}$ approaches the effective power $P_{2}$. If we take, for the coefficient of load, the figure $3 / 2$ already mentioned, the maximum power $P$ should not be less than $3 / 2 \times 37.5=56 \mathrm{k}$.w., which corresponds to a line-resistance of about I 6 ohms.

In his experiments Mordey (Proc. Inst. of Electr. Eng., I894) was able to increase the resistance to very much higher values (I $40 \mathrm{ohms}$ ), 
because he made his machines run without load. This proves nothing, however, in regard to their operation under load. Moreover, these conditions were absolutely exaggerated, since lines of such high resistance would never be used in practice.

Finally, when we attain a resistance of approximately $R=\frac{86}{\sqrt{3}}=50$ ohms, we have $\cos \phi=\frac{1}{2}$. The maximum power for $E_{2}=E_{1}$ being then no longer 22,550 watts, the output of the motor can scarcely exceed 15 k.w. Besides, it is sure that every increase of the E.M.F. $E_{2}$ above 2200 volts will diminish the stability. This will be all the more true if $R$ is higher. We can thus understand that in using machines of $50 \mathrm{k} . \mathrm{w}$. with an external or line resistance of $63 \mathrm{ohms}$, Mordey could only operate these machines without load, and that the slightest increase of the voltage $E_{2}$ above $E_{1}$ (20 per cent) would cause the machines to fall out of step.

The preceding analysis therefore explains perfectly the anomalies of Mordey's experiments.

We would be brought to analogous conclusions by the consideration of sudden overloads and of oscillating changes of load, which will be discussed later.

[The amounts of sudden overloads allowable are discussed in the author's preceding work, "The Coupling of Alternators," and also in La Lumicre Electrique, Vol. XLV, p. 474.]

\section{COMPARISON OF POSSIBLE OUTPUTS AT CONSTANT LOAD WITH VARIOUS EXCITATIONS. CONSTANT POTENTIAL SUPPLY}

Existence of a Current-Minimum. There exist several values of current and E.M.F. which permit a motor subjected to an external constant E.M.F. $E_{1}$ to develop a given electrical power $P_{2}$. These are all values for which the point $A_{2}$ (Fig. 27) falls on the line of equal power corresponding to that value $P_{2}$.

These values differ from each other by the values of the E.M.F. $E_{2}$ necessary to produce them, and also by the phase-angles $\theta$ and $\phi$ of the corresponding current $I$, and, particularly, by the reactive current.

If, to simplify matters, we suppose the motor to be supplied from a constant potential source whose impedance is negligible, the diagram in Fig. 27 will give the solution.

The current being represented by the vector $A_{1} A_{2}$ according to the scale of amperes, its minimum evidently corresponds to the point 
$F$ on the power-circle $G G^{\prime}$, situated on the phase-line $A N$. As soon as the point $A_{2}$ is displaced on the circle to either side of the line $A N$, the current increases. There is therefore a value of the internal E.M.F. $E_{2}$ which corresponds to a minimum current, and all values greater or less than this value necessitate a stronger current.

It is evident from Fig. 27, without any explanation, that the minimum value is that which brings the current into phase-coincidence with the E.M.F. $E_{1}$, i.e., with the E.M.F. at the terminals, since the motor is supposed to be supplied from a constant potential source having negligible impedance.

[This condition gives, immediately, for the corresponding algebraical value of $E_{2}$ the following

$$
E_{2}^{2}=E_{1}^{2}+(Z I)^{2}-2 E_{1} R I .
$$

Suppose, for example, a $40 \mathrm{k}$.w. motor connected to a 2000 -volt circuit, its efficiency being $\eta=0.85$, and the constants being $R=2 \mathrm{ohms}$, and $Z=40 \mathrm{ohms}$. The minimum current necessary, at full load, will be about

$$
I=\frac{40,000}{0.85 \times 2000}=23.5 \text { amperes; }
$$

whence

$$
Z I=940 \text { volts. }
$$

The corresponding E.M.F. will be

$$
E_{2}=\sqrt{(2000)^{2}+(940)^{2}-2000 \times 47 \times 2}=2 \text { r } 66 \text { volts. }
$$

The corresponding value of the exciting current will be obtained by reference to the excitation-curve of the alternator.

V-Curves. The foregoing conclusions, which had already been formulated by the author in a paper in $189^{2}$ ( $L a$ Lumiere Electrique,

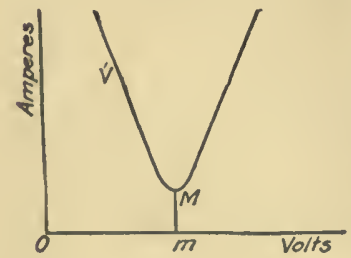

Fig. 30 .

Vol. XLV, pp. 423 and 563 ), were confirmed experimentally by Mordey in 189.3 . Mordey's results were represented graphically by a curve, in rectangular co-ordinates, to which the name of $\mathrm{V}$-curve has been given, owing to its characteristic appearance (Fig. 30). This curve is obtained by taking as abscissx the values of the E.M.F. $E_{2}$ and as ordinates the corresponding current-values. It is readily seen that the current does pass through a minimum $M \mathrm{~m}$ for a certain value $O m$ of the voltage. 
Use of Diagram of the First Kind. The diagram of the first kind lends itself more rigorously than the other to the study of voltage and current values when the motor is working at constant load; and it also applies equally well in the case where the impedance of the generator can be neglected.

It is sufficient to cause the segments $O A_{2}, O^{\prime} A_{2}, O^{\prime \prime} A_{2}, O^{\prime \prime \prime} A_{2}$, (Fig. ${ }_{3}$ I), representing the E.M.F. $E_{2}$, to vary in inverse ratio with respect to the segments $A_{2} D, A_{2} D^{\prime}, A_{2} D^{\prime \prime}$, representing the active current $I_{w}$, in such manner that the product $E_{2} I_{w}$ shall be constant and equal to the given power $P_{2}$. If, from each point, $O, O^{\prime}, O^{\prime \prime}$, an arc of circle

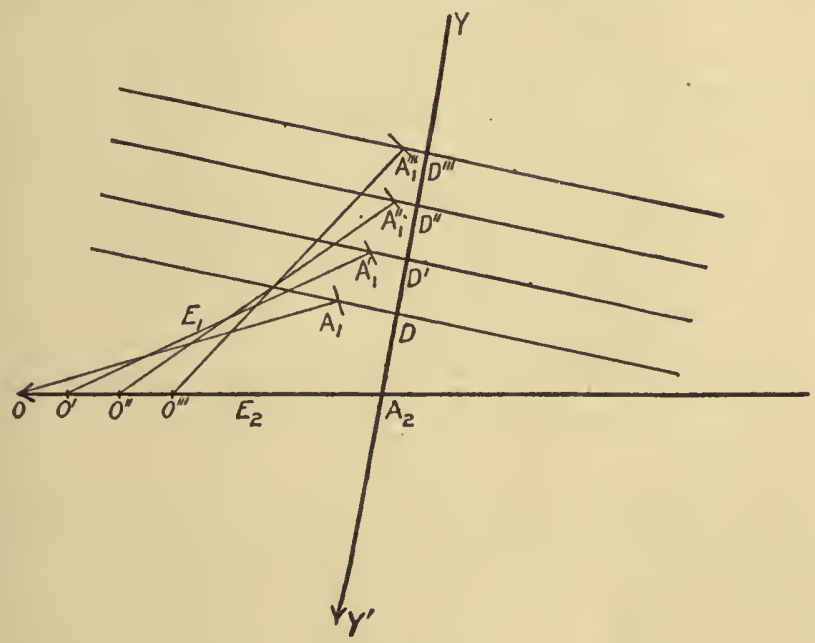

FIG. 3I.

is described, having for its radius the generator E.M.F. $E_{1}$, their respective intersections with the lines passing through $D, D^{\prime}, D^{\prime \prime}, D^{\prime \prime \prime}$ and perpendicular to $A_{2} Y$, determine the load-points $A_{1}, A^{\prime}{ }_{1}, A^{\prime \prime}{ }_{1}, A^{\prime \prime \prime}{ }_{1}$; and, consequently, the current-values $I$ corresponding to the various values of $E_{2}$. This diagram also applies in the case where $E_{1}$ varies according to some given law.

Predetermination of V-Curves. The preceding diagrams enable these curves to be obtained by a simple graphical transformation, without calculation. It is sufficient to measure, on the diagram itself, the radial vectors for corresponding values of E.M.F. and current, and to plot them as rectangular abscissæ and ordinates. Each of the power-lines of Fig. 28, for example, also gives a transformed curve 
which is the corresponding V-curve (Fig. 32). These curves show clearly that the $\mathrm{V}$ becomes more rounded as the corresponding power $P_{2}$ increases. This fact is, moreover, verified by experience, as shown,

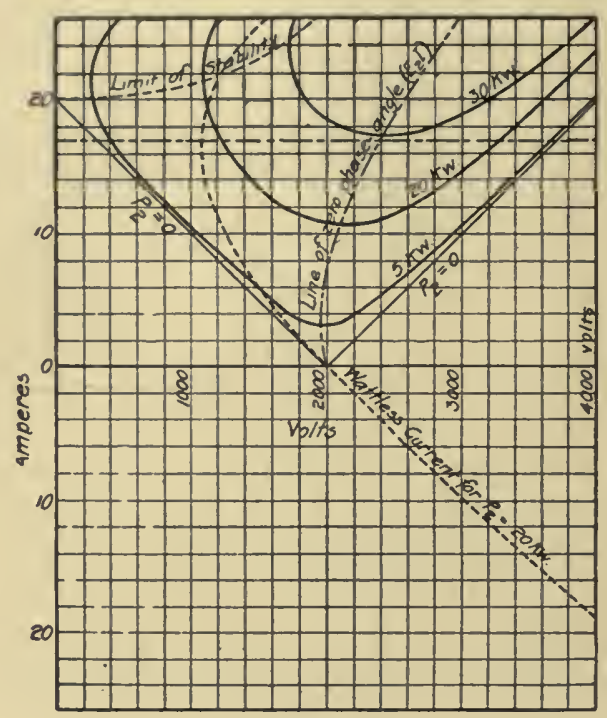

FIG. 32.

for example, by the curve in Fig. 33, obtained experimentally from a synchronous motor of Labour type running at constant load of $5 \mathrm{k}$. w. (half load).

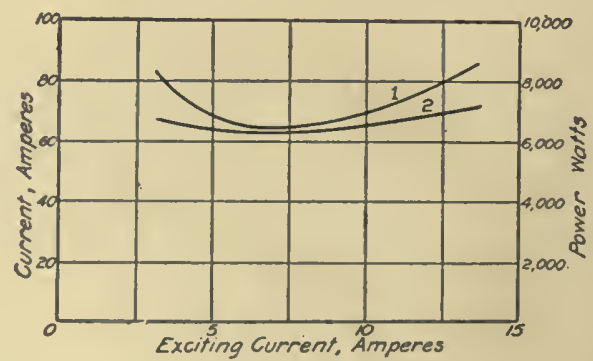

Fig. 33.

The sharpest $\mathrm{V}$ is obtained with no load. Theoretically, the curve $P_{2}=$ o (Fig. 32) ought to present an angular point at the axis of amperes; but, in practice, the power consumed by friction, eddy currents etc., when running without load, is not negligible, and, for these rea- 
sons, the curve of no-load has a form analogous to the $5 \mathrm{k}$.w. curve of this figure.

It will be noticed that, in practice, it is possible to obtain only a rather limited portion of curve, under load, because the values become more and more unstable as they depart from the minimum current value, either way.

Theoretical Form of V-Curves. Although, for various reasons considered later, the $\mathrm{V}$-curves always differ slightly in practice from those deduced from the diagram, it is interesting to determine their theoretical algebraical equation.

If we note that the current $I$ and the power $P_{2}$ are given, by eqs. (4) and (8), in terms of $E_{1}, E_{2}, \gamma$, and $\phi$, it will be seen that we only need to eliminate $\phi$ between these two equations to obtain the desired relation between $E_{2}$ and $I$.

From eq. (4), expanding and replacing $\sin \gamma$ and $\cos \gamma$ by their values $\frac{X}{Z}$ and $\frac{R}{Z}$, we have

$$
\left.\begin{array}{rl}
E_{1}{ }^{2} & =E_{2}^{2}+(Z I)^{2}+2 E_{2}[Z I \cos \phi \cos \gamma+Z I \sin \phi \sin \gamma] \\
& =E_{2}^{2}+(Z I)^{2}+2 P_{2} R+2 X \sqrt{E_{2}^{2} I^{2}-P_{2}^{2}}
\end{array}\right\} .
$$

The equation obtained on squaring to remove the radical sign is

$$
\left[(Z I)^{2}+E_{2}^{2}-E_{1}^{2}+{ }_{2} P_{2} R\right]^{2}-4 X^{2}\left(E_{2}^{2} I^{2}-P_{2}^{2}\right)=0 .
$$

This equation is of the $4^{\text {th }}$ degree in $I$ and in $E_{2}$. The $V$-curves obtained, in rectilinear co-ordinates, taking $E_{2}$ as abscissæ and $I$ as ordinates, are therefore more or less complicated curves of the $4^{\text {th }}$ degree. They may be calculated by points by solving with respect to $I$, for example, but the calculation is unnecessarily complicated; and the graphical construction shown in Fig. $3 \mathrm{I}$ is preferable.

In the particular case where $P_{2}=0$ the equation reduces to

$$
(Z I)^{2}+E_{2}^{2} \pm 2 X E_{2} I=E_{1}^{2},
$$

i.e., an assemblage of two easily constructed ellipses whose axes intersect each other as shown in Fig. 34. The V-curve for no load is therefore formed of two branches of an ellipse which intersect each other on the axis of amperes. The sections of it which are in the useful portion are almost rectilinear. 
[Since there is no term of the first degree, these ellipses have the origin of the co-ordinates for their center. If we take as ordinates the product $Z I$, the inclination of their axes is constant and equal to

$$
\frac{Z I}{E_{2}}=\tan 45^{\circ}=\mathrm{I} \text {. }
$$

If, instead of the product ( $Z I)$, we take, as ordinates, the values of $I$, the inclination of their axis becomes

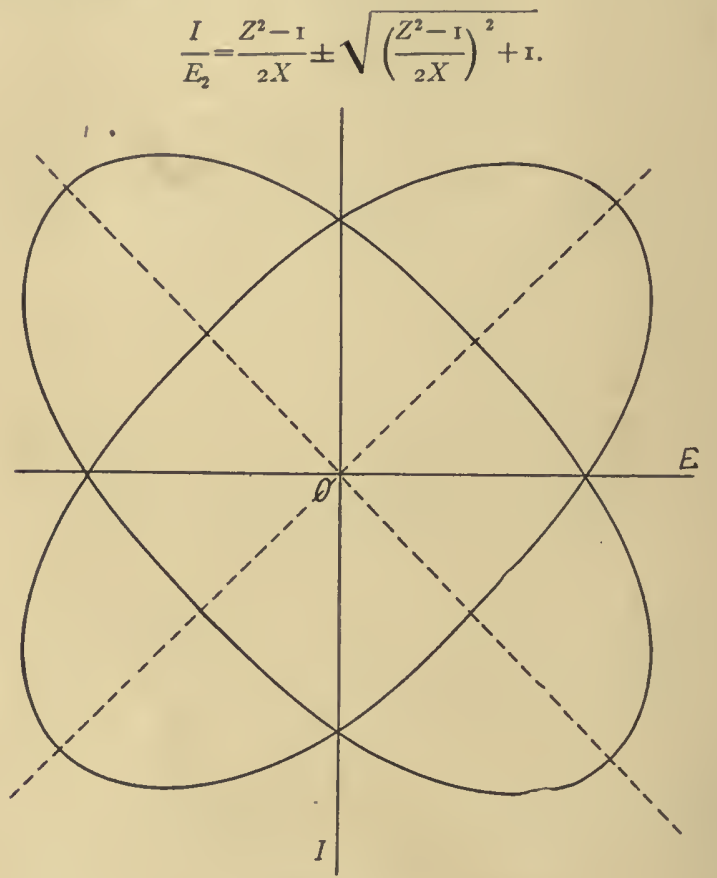

FIG. 34.

This inclination increases inversely as the reactance $X$, i.e., a given variation of the exciting current produces a current-variation which is all the greater the higher the inductance of the motor. This conclusion is supported by other considerations, as we have already seen.]

The conditions for which the current is a minimum are those for which the phase is zero with respect to $E_{1}$. They are obtained by taking, on the diagram of Fig. 27 , all the values situated on the right line $A_{1} N$. For these points the triangle $O A_{1} A_{2}$ gives

$$
\begin{aligned}
E_{2}^{2} & =E_{1}^{2}+(Z I)^{2}-2 E_{1} Z I \cos \gamma \\
& =E_{1}^{2}+(Z I)^{2}-2 E_{1} R I .
\end{aligned}
$$


In rectangular co-ordinates the relationship between $E_{2}$ and $I$ is represented by the hyperbola ${ }^{1}$ passing through the point of intersection of the two arcs of the ellipse of zero-power, and having its principal axis horizontal and situated above the axis of abscissæ at a height equal to

$$
I=\frac{R E_{1}}{Z^{2}}
$$

The excitation which renders the current a minimum must therefore decrease at first when the power required, $P_{2}$, increases; and it must then increase more and more. The greater the impedance the sooner this increase will occur. These results are confirmed by experience, but, in practice, this critical value of $E_{2}$ may be considered constant.

Curve of Reactive Current. Fig. 32 would be incomplete if only the total current $I$ were represented therein, because the diagram would not then give information in regard to the phase of the current. It is therefore quite useful to add, to the $\mathrm{V}$-curves, other curves indicating the value of the corresponding positive or negative reactive current. The values of the reactive current are deduced from the diagram by constructing the lines corresponding to each value of the phase-angle 0 . The curve of reactive current corresponding to $P_{2}=20 \mathrm{k} . \mathrm{w}$. has been drawn as an example in Fig. 32. In practice this curve can be plotted from measurements made by a wattmeter.

Expression for Reactive Current. The expressions for currents which are reactive with regard to $E_{1}$ or $E_{2}$ can be calculated in like manner. The reactive current as a function of $E_{2}$ will be obtained by starting from eq. $(m)$. This equation can be written

$$
E_{1}^{2}=E_{2}^{2}+Z^{2}\left[I_{d}^{2}+\left(\frac{P_{2}}{E_{2}}\right)^{2}\right]+{ }_{2} P_{2} R+2 E_{2} X I_{d}
$$

This is also an equation of the $4^{\text {th }}$ degree in $E_{2}$, but it can be easily solved with respect to $I_{d}$, if written in the following form:

$$
Z^{2} I_{d}{ }^{2}+2 X E_{2} I_{d}-\left(E_{1}{ }^{2}-E_{2}{ }^{2}\right)+\left(\frac{Z P_{2}}{E_{2}}\right)^{2}+2 P_{2} R=\text { 。 }
$$

1 The conditions of zero phase-angle of the current with respect to the E.M.F. $E_{2}$ would be the same on an ellipse such as

$$
E_{1}^{2}=E_{2}^{2}+(Z I)^{2}+2 E_{2} R I
$$

but they present no special interest. 
For the particular case $P_{2}=0$, this equation reduces to

$$
Z^{2} I_{d}^{2}+2 X E_{2} I_{d}+E_{2}^{2}=E_{1}^{2}
$$

which represents one of the ellipses of zero-load already determined.

The reactive current $j$, with respect to $E_{1}$, is obtained by writing the relation between $E_{2}$ and $E_{1}$, as it is depicted in Fig. 26, thus

$$
E_{2}^{2}=E_{1}^{2}+(Z I)^{2}-2 E_{1} Z(i \cos \gamma+j \sin \gamma),
$$

or

$$
E_{2}^{2}=E_{1}^{2}+Z^{2}\left[\left(\frac{P_{2}}{E_{2}}\right)^{2}+j^{2}\right]-2 E_{1}\left(R \frac{P_{2}}{E_{2}}+X j\right)
$$

With no load, when $P_{2}=0$, this equation reduces to

$$
E_{2}^{2}=E_{1}^{2}+Z^{2} j^{2}-2 E_{1} X j
$$

The curve of $j$ as a function of $E_{2}$, drawn in rectangular co-ordinates, would therefore be a hyperbola; we can write

$$
E_{2}{ }^{2}-\left(Z j-\frac{E_{1} X}{Z}\right)^{2}=E_{1}{ }^{2}\left(1-\frac{X^{2}}{Z^{2}}\right) .
$$

The horizontal axis is situated at a distance

$$
\frac{E_{1} X}{Z^{2}}
$$

above the axis of volts.

These formulx enable the reactive currents to be calculated algebraically with more precision, but also much more laboriously, than by the graphical method.

Comparison of Outputs which the Same Alternator Can Develop with the Same Terminal Voltage, when Used Either as a Generator or as a Motor. Two different cases have to be considered:

(I) Let us suppose the case of an alternator connected to a constant potential system of great power, presenting a negligible impedance, and let the excitation be regulated in such a manner as to give the minimum current. Assuming a sufficient margin for regulating the excitation, the output of the machine will be limited in all practical cases by the value of the armature-current. 
Let $r$ cqual the armature-resistance, $x$ the armature-reactance, $z$ the armature-impedance, $U$ the voltage at the terminals, $I$ the maximum allowable current. The internal E.M.F. of the corresponding generator, necessary to produce this current, will be obtained (Fig. 35 ) by combining the vector $U$ with a vector $A_{1} A=z I$ drawn below $U$ at an angle $=r$, such that $\tan \gamma=\frac{x}{r}$. In order that the same machine, when operating as a motor, at the same voltage, $U$, may furnish the maximum power, $U I$, with the same current, $I$, the latter must have the same difference of phase, $\gamma$, with respect to $U$, but in the opposite direction. The internal E.M.F., $E_{2}=O A_{2}$, will therefore be obtained by compounding $O A$ with a vector $z I$ equal, but opposed, to the preceding one.

Fig. 35 thus shows that the alternator corresponds to the same

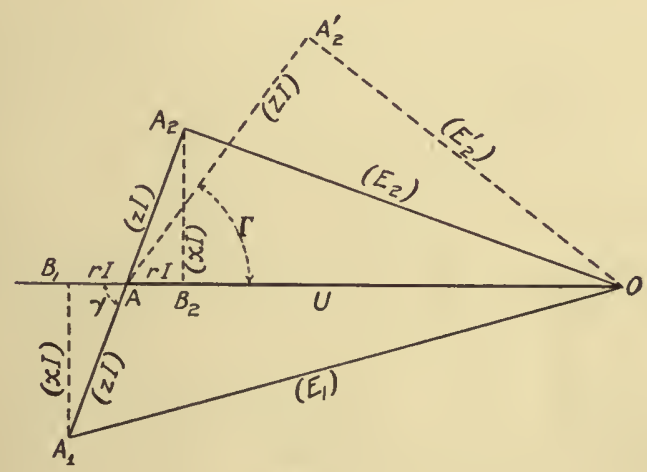

FIG. 35.

electric power at its terminals when operating as a motor and as a generator, and this with substantially the same excitation. Assuming the losses to be the same in both cases, it is seen that by increasing slightly the F.M.F. $E_{2}$, the same normal power may be counted upon for a motor as for a generator. (The normal power here designated is the power which the alternator can furnish on a dead resistance. This is the figure given in the catalogues of manufacturers of synchronous motors.)

(2) If now we consider the case where power is to be transmitted to a distance, the impedance $Z$ which counts in the operation of the motor is that of the entire circuit and it can be notably superior 
to the impcdance of the armature. The segment $Z I$ then extends as far as $A^{1}{ }_{2}$, for example, making an angle $\Gamma$, which is such that $\tan \Gamma=\frac{X}{R}$; and the E.M.F., $O A_{2}^{\prime}$, necessary to obtain this output may be superior to the preceding value. Eq. (25) gives the value thereof. It is necessary to foresee this condition, in studying an electric transmission project, whenever the impedance of the line is of the same order as that of the alternators.

I IV. INFLUENCE OF MOTORS ON THE GENERAL OPERATION OF AN A.C. ELECTRICAL DISTRIBUTION SYSTEM

Effect of Current of Synchronous Motors on Distribution-Systems. There is a tendency, at the present time, and with good reason, to use synchronous motors under conditions which permit them, as if they were condensers, to compensate for the inductance of neighboring motors. This method, which consists in overexciting the synchronous motors, whether they be utilized for the production of mechanical power, or whether they be running without load, appears to have been first advocated in I89I by Swinburn; and it has been used since by many engineers in Europe and in America. Following the advice of DolivoDobrowolsky it was used for the Bulach-Oerlikon power-transmission in 1893 (see letter of Dolivo-Dobrowolsky to the Elektrotechnische Zeitschrift, 4. Oct., 1894, p. 555); and Lahmeyer also used it at Bockenheim (see Deutscher Verband der Elektrotechnischen Ingenieure, Congress of June, I894), where he was thus enabled, it would seem, to double the output of the generators by compensating all inductanceeffects in the distributing system by the reaction of synchronous motors. Some time after, Professor S. P. Thompson called attention to this method ("Some Advantages of Alternating Currents", by Professor S. P. Thompsun, British Association, I894).

To study this question, we must ascertain how the reactive current varies with respect to the voltage at the terminals of the generator.

A polar diagram referred to the line-voltage as a fixed axis gives the necessary values most accurately.1 Let us take (Fig. 27, p. 44)

1 We use here this form of diagram to take the ohmic loss of the motor into account. In practice, the latter can be neglected without great inconvenience, and the diagram referred to the motor E.M.F. $E^{2}$, which is more simple, will answer the purpose. We can then see more readily that the conditions corresponding to 
on a power-line, $P_{2}$, two output-points, $A_{2}$ and $A_{2}^{\prime}$, situated respectively at the right and at the left of the phase-line $A_{1} N$; and let us project them on that line, at $a$.

From what has already been seen, the lengths $\overline{A_{2} a}$ and $\overline{A_{2}^{\prime} a}$ will represent the values of the reactive currents corresponding to the two outputs; but while the first, $\overline{A_{2} a}$, is lagging with respect to the working current, which is equivalent to the effect of a self-induction with all of its objections, the second, $\overline{A_{2}{ }^{\prime} a}$, is leading, which is equivalent to the effect of a condenser.

We therefore see that for all values whose representative point is situated on the left of the phase-line $A_{1} N$ there will be a reactive leading current. It is this current, equivalent to that of a condenser, which enables the lagging reactive current to be neutralized, including the magnetization-current necessary for various translating appliances, such as induction-motors, transformers, induction-coils, etc., on the same portion of the circuit.

As we shall see later, it is very easy to calculate these compensationphenomena with a view to their practical application.

In studying the effects produced by the reaction of synchronous motors, we should, at the outset, distinguish between two very different things, i.e., the load (current) on the line and the load (current) on the generating station supplying that line.

(x) Compensation with Respect to the Line or Circuit. Many ordinary translating appliances, notably transformers, arc-lamps, and especially induction-motors, have a power-factor, $\cos \phi$, which is lower than unity, and which increases uselessly the heating of the conductors of the distributing system or of the line, in the case of a simple transmission-system. [The loss of voltage in the line is likewise increased in greater proportion than the current, in consequence of lag, as the author has shown elsewhere. See articles on "Line-inductance for Alternating Currents", l'Eclairage Electrique. Oct., Dec., x894.]

The aim of the compensation sought should be to increase this porver-factor as much as possible, by producing the reactive currents locally instead of transporting them over the line.

The evaluation of these currents is not difficult, owing to the fact that in the translating appliances just mentioned the magnetizing

points situated on the left of the reference-line $O Y$ are those which produce a leading current with respect to the voltage at the terminals. It is therefore on the left of $O Y$ that the motor plays the role of a condenser. 
(or reactive) currents are practically constant at all loads and can even be predetermined, at least roughly, according to the output of the appliances, without knowing exactly what these are. Let $J_{d}$ be the total value of the reactive currents, and $J_{w}$ be the total corresponding active current, at any given instant.

To neutralize completely the lag of the line, it would be necessary to produce, by means of the synchronous motors in- that part of the line, a reactive current which is leading in phase and equal to

$$
j=J_{d},
$$

(in absolute units). But it is not usually necessary to fulfill strictly this condition, because it may be, as a rule, considered sufficient to have a power-factor 0.95 to 0.97 , corresponding to reactance-factors $(\tan \gamma)$ ranging in value from $\frac{1}{3}$ to $\frac{1}{4}$, respectively. Let us take, for example, $\tan \gamma=\frac{1}{3}$. It is then sufficient to satisfy the equation

$$
\frac{J_{d}-i}{J_{w}+i}=\frac{1}{3},
$$

in which $i$ equals the active current absorbed by the synchronous motors, and which must be added to that of the apparatus, $J_{w}$ (useful load).

From this we have

$$
j=\left[J_{d}-\frac{1}{3}\left(J_{w}+i\right)\right],
$$

This means that the reactive current which the synchronous motors must produce to render the reactance-effects of neighboring motors practically negligible need not be equal to the current actually consumed by these appliances, but may be reduced more and more as their useful load $J_{w}$ is increased. Owing to this circumstance, one or more relatively small synchronous motors will suffice, in practice, to neutralize the harmful effects of reactance on the line.

Let us suppose, for simplicity, that there is only one such compensator-motor, and that it is also serving, at the same time, as a motor doing mechanical work. Its total armature-current will be equal to the resultant

$$
I=\sqrt{i^{2}+j^{2}}=i \sqrt{\mathrm{I}+\left(\frac{j}{i}\right)^{2}} .
$$

The current $I$ being limited by the heating of the motor, the useful current $i$ and, consequently, the useful corresponding power $P_{2}$, will 


\section{DETAILED STUDY OF OPERATION WITH NORMAL LOAD 67}

have to be all the lower the greater the value of $j$. If it is satisfactory for example, to take $j=\frac{1}{3} i$, the increase of $I$ will be almost negligible.

The graphical determination of $I$ and $E_{2}$, is obtained readily from the fundamental diagram (Fig. 26) by construçting (Fig. 36), according to the scale of volts, the lines $Z i$ in the $\operatorname{dircction} A_{1} N$, and $Z_{j}$ in the perpendicular direction, and by drawing $A_{1} A_{2}$ and $O A_{2}$. If $I$ and $j$ are known beforehand, $E_{2}$ and $i$ can be deduced inversely. [All that is

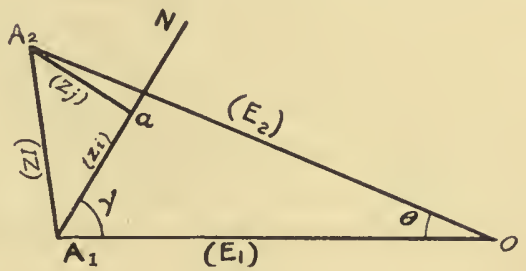

FIG. 36 . necessary is to project the broken line $A_{2} A_{1} O$ on the line $A_{1} N$ and on the line perpendicular thereto; the two projections are

and

$$
Z i-E_{1} \cos \gamma
$$

$$
Z j+E_{1} \sin \gamma \text {. }
$$

The sum of their squares gives $E_{2}{ }^{2}$, from which we deduce the formula, (26)].

This diagram also gives the algebraical expression of $E_{2}$. thus

$$
E_{2}=E_{1} \sqrt{I+\left(\frac{Z I}{E_{1}}\right)^{2}+\frac{2 Z}{E_{1}}(j \sin \gamma-i \cos \gamma)}
$$

From this, given the excitation-curve of the motor, the necessary ampere-turns can be deduced.

In order that the compensation may be really advantageous for the line, it is necessary that the resultant currents should be notably weaker than before, i.e., we must have

$$
\sqrt{\left(J_{w}+i\right)^{2}+\left(J_{d}-j\right)^{2}}<\sqrt{I_{w^{2}}+J_{d}^{2}} .
$$

In practice, compensation of this kind is especially advisable when synchronous apparatus (motors or converters) is already connected on the distribution-system, and serves for other purposes; because, all that is then necessary is to increase slightly the excitation-current of each of them, which involves no special expenditure of energy other than a slight supplementary loss in the fields and in the armature. The companies which operate alternating-current systems should therefore 
seek to encourage as much as possible the use of such apparatus by their patrons.

When no such apparatus exists on the system, it may be desirable to place synchronous motors running without load at the distributing centers for the special purpose of furnishing reactive current. Let us see what the nominal power of such a motor should be.

The reactive current $i$ being then only the current necessary to keep the apparatus in motion, if we designate by $p$ the sum of the losses without load (i.e., friction, hysteresis, eddy currents and power necessary for excitation), we will have, approximately,

$$
i=\frac{p+r I^{2}}{E_{1}}
$$

If the apparatus is efficient $i^{2}$ will be very small with respect to $j^{2}$ and consequently we can write, as an approximation,

$$
I=j \text {. }
$$

[In a good motor the active current with no load, $i$, does not exceed 0.10 or 0.20 of the full-load current. The lag of the current of an overexcited motor running without load can therefore approach very near $\frac{\pi}{2}$, without nevertheless approaching as closely thereto as would the current of a condenser.]

Since 0 is very small, under these conditions, $E_{2}$ becomes approximately

$$
E_{2}=E_{1}+Z j
$$

The power for which the motor is rated by the manufacturer is equal to the product of the ordinary E.M.F. (usually with a margin of I 5 to 25 per cent increase), by the normal current. For example, under this hypothesis, it would be possible to use a motor having a catalogue voltage of $\frac{E_{2}}{1.25}\left(\right.$ more generally, $\left.\frac{E_{2}}{1+\varepsilon}\right)$ and having the normal current $I$. Its rated power will be

$$
P_{1}=\frac{\mathrm{I}}{\mathrm{I} \cdot 3 \mathrm{O}} j\left(E_{1}+Z_{j}\right)
$$

in which $j$ has the value already given. This value of $j$ shows that, in an installation comprising a certain number of machines in permanent operation, the capacity of synchronous motor necessary for compensation may be taken lower in proportion as the load-factor will be higher, 
and that, if sufficient compensating effect has been obtained at light loads, the compensating effect will be still better with heavier loads.

First Numerical Example. Assume a distribution-system supplying $500 \mathrm{k} . \mathrm{w}$. of transformers at 5000 volts. The magnetizing current is a small fraction of the full-load current, varying usually from 2 per cent to 5 per cent, according to whether the transformers are large or small. Let the average be taken at 3.5 per cent. The full-load current being 100 amperes, the total magnetizing current, which is approximately constant at all hours of the day, will be 3.5 amperes. The powerfactor with no load being, on the average, 0.70 , the active current with no load will also be 3.5 , and the resultant current will be $\frac{3 \cdot 5}{0.7}=5$ amperes. To neutralize the lag by means of a synchronous motor having a rated efficiency of 0.80 when running without load, it would be necessary to produce a reactive current of 3.5 amperes at the expense of 0.7 ampere of active current corresponding to the resistance-losses. The current taken from the distribution-system would then be $3.5+0.7=4.2$ amperes, instead of 5 amperes. This saving is not sufficient to justify the purchase and maintenance of a motor intended to serve solely as a compensator; but the use of a condenser would not be much more advantageous, as the current would always remain slightly above 3.5 in consequence of dielectric hysteresis.

Second Numerical Example. Assume a distribution-system comprising, at the end of the line, where the tension is 2000 volts, some translating devices composed of $200 \mathrm{k} . \mathrm{w}$. of induction-motors and 100 k.w. of synchronous motors, the latter having a total reactance of 15 ohms and a resistançe of $0.8 \mathrm{ohm}$. Assume the synchronous motors to have an efficiency of 80 per cent, the induction-motors to have a magnetizing current equal to one-third of the normal current, an efficiency of 85 per cent, and a power-factor of 0.95 , at full load.

[The magnetizing current generally varies between one-third and one-quarter of the loar-current in polyphase motors and between onehalf and one-third in single-phase motors.]

The problem assumed is: to compensate the effects of the lagging current in the induction-motors when running with no load, and also with a quarter-load, it being assumed that all the motors are constantly in operation.

The total current necessary for the induction-motors at full load is

$$
\frac{200000}{0.85 \times 0.95 \times 2000}=\mathrm{I} 24 \text { amperes. }
$$


The reactive current will be, at full load, approximately

$$
=\frac{124}{3}=42 \text { amperes. }
$$

It can be assumed that the corresponding active current, at no load, is Io per cent of the total, or 12 amperes.

Since the synchronous motors, in this case, run without load, it would be necessary, in order to compensate absolutely for the phaselag, to make them produce also a reactive current

$$
j=42 \text { amperes; }
$$

and since their active current attains, at most,

$$
i=\frac{0.20 \times 100000}{2000}=\mathrm{I} 0 \text { amperes, }
$$

these motors will take a maximum current equal to

$$
I=\sqrt{(10)^{2}+(42)^{2}}=42 . I \text { amperes, }
$$

while their normal-load current might attain

$$
\frac{100000}{0.80 \times 2000}=62.5 \text { amperes. }
$$

The E.M.F. is given by the approximate formula

$$
E_{2}=2000+\left(\mathrm{I}_{5} \times 42\right)=2630 \text { volts. }
$$

The line-current, which, without compensation, would be

$$
\sqrt{(10+12)^{2}+(42)^{2}}=50.5 \text { amperes }
$$

(or at least 42.1 amperes, if the synchronous motors were not operating) would thus be reduced, by the compensation, to

$$
10+12=22 \text { amperes. }
$$

It might even be reduced to 19 amperes, if only two-thirds of the synchronous motors were being operated. 
Let us suppose, now, that each group of motors shall furnish 50 k.w. The active current for the induction-motors can be estimated at 25 amperes, plus 8 amperes for losses, or in all 33 amperes; and the active current for the synchronous motors can be estimated at 25 amperes, plus the losses, which are at most ro amperes, or, in all, 35 amperes.

The total active current is therefore $33+35=68$ amperes, while the reactive current of the induction-motors remains, as before, 42 amperes. The resultant current is

$$
\sqrt{(42)^{2}+(68)^{2}}=92.5 \text { amperes, }
$$

and it could not be less than 68 amperes.

Suppose it be required to bring up the power-factor to 0.95 , i.e., to bring the current down to 72 amperes. It will be sufficient to reduce the reactive current to $\frac{68}{3}=23$ amperes by producing a current out of phase which is equal to

$$
j=42-23=19 \text { amperes. }
$$

The current of the synchronous motors will then become equal to about

$$
\sqrt{(I 9)^{2}+(35)^{2}}=40 \text { amperes }
$$

instead of 35 (i.e., it would be slightly-increased). We will have $\tan \gamma=\frac{19}{35}=0.543$ and the E.M.F. will be equal to

$$
\begin{aligned}
E_{2} & =2000 \sqrt{\mathrm{I}+\left(\frac{\mathrm{I} 5 \times 40}{2000}\right)^{2}+\frac{15 \times 2}{2000}(19 \times 0.477 \mathrm{I}-35 \times 0.8788)} \\
& =1728 \text { volts. }
\end{aligned}
$$

instead of 1486 volts, which the motor should develop, when the phaseangle $r$ equals zero.

It is seen that, by overexciting to a maximum of 30 per cent, it is possible to compensate for the full-load reactions, without overloading the synchronous motors. To obtain the same result by means of a motor running without load it would be necessary to use an apparatus having a rating of about $40 \mathrm{k.w}$.; which would not be very practical. 
Economic Study of Compensation for the Line by Means of Motors Running without Load. For simplicity, let us suppose the following to be constant: the voltage at the sending and receiving ends, the power to be transmitted, and the loss of energy in the line. It is required to determine the economy resulting from compensation by overexcited synchronous motors running without load, for the different values of $\cos \phi$.

Two points have to be considered:

(I) The saving in the capital invested;

(2) The saving in annual cost of operation.

These two points lead, as will be seen, to conclusions which are appreciably different:

(I) Saving in Cost of Equipment. Let (for a singlè-phase current), $E_{0}=$ the voltage at the transmitting end of the line; $E_{1}=$ the voltage at the receiving end; $E_{2}=$ the E.M.F. of the synchronous motors; $I_{w}=$ the useful active current; $i=$ the active current of the synchronous motors; $I_{d}=I_{w} \tan \phi=$ the reactive current when there is no compensation; $j=$ the reactive current of the synchronous motors; $I_{1}=$ the resultant current of the synchronous motors; $p_{0}=$ the cost of the generators per kilovolt-ampere; $p_{1}=$ the cost of the synchronous motors per kilovolt-ampere; $p_{2}=$ the price of line-copper per kilovoltampere transmitted.

The price of line-copper, when there is no compensation, is given by the formula

$$
p_{2} \frac{E_{1} I_{w}}{\cos \phi}
$$

(which includes the return-conductor). In this formula the factor $p_{2}$ is equal to

$$
p_{2}=65 \times b \frac{1+\varepsilon}{\dot{\varepsilon}} \times \frac{l^{2}}{E_{0}^{2}} \times 10^{4}
$$

wherein $l=$ length, in kilometers; $b=$ price of copper per kilogram, (assumed $=2.50$ francs).

$\varepsilon=$ the percentage of loss allowed in the line; $E_{0}=$ the initial E.M.F. in volts.

The voltage being supposed constant at the transmitting and receiving ends, it follows that, in order to transmit the same power with the 
same losses in the line for the different values of $\cos \phi$, the weight of the line-copper will have to vary.

The cost, in francs, ${ }^{1}$ of the line-copper, when there is no compensation, is

$$
p_{2} \frac{E_{1} I_{w}}{\cos \phi}
$$

and, when there is compensation; it is

$$
\frac{\left(I_{w}+i\right)^{2}+\left(I_{w} \tan \phi-j\right)^{2}}{\frac{I^{2}}{\cos ^{2} \phi}} \times p_{2}{ }^{\prime \prime} \frac{E_{1} I_{w}}{\cos \phi} .
$$

The cost of the generators, without compensation, is

$$
p_{0} \frac{E_{0} I_{w}}{\cos \phi}
$$

and, with compensation,

$$
\sqrt{\frac{\left(I_{w}+i\right)^{2}+\left(I_{w} \tan \phi-j\right)^{2}}{\frac{I_{w}}{\cos \phi}}} \times p_{0} \frac{E_{0} I_{w}}{\cos \phi} .
$$

Finally, the cost of the synchronous motors is

wherein

$$
p_{1} E_{1} I_{1}
$$

$$
I_{1}=\sqrt{i^{2}+j^{2}} \text {. }
$$

The total saving in cost of equipment, as the result of compensation, will be (in francs) ${ }^{1}$

$$
\begin{aligned}
p_{2} \frac{E_{1} I_{w}}{\cos \phi}\left[\mathrm{I}-\frac{\left(I_{w}+i\right)^{2}+\left(I_{w} \tan \phi-j\right)^{2}}{\left(\frac{I_{w}}{\cos \phi}\right)^{2}}\right]+ \\
\quad+p_{0} \frac{E_{0} I_{w}}{\cos \phi}\left[\mathrm{I}-\frac{\sqrt{\left(I_{w}+i\right)^{2}+\left(I_{w} \tan \phi-j\right)^{2}}}{\frac{I_{w}}{\cos \phi}}\right]-p_{1} E_{2} I_{1} .
\end{aligned}
$$

1 The corresponding cost value in dollars will be given by the same formula by introducing the factor 0.20 in the formula. The same remark applies to all the formulæ which follow in which cost-values are given in francs. 
(2) Saving in Annual Operating Cost. The annual operating expenses include depreciation and interest on the capital invested, and the cost of the extra energy expended in the generators and synchronous motors.

The energy expended in the line is supposed to be the same with or without compensation.

Let us assume the following figures:

Depreciation of copper ........... 3 per cent;

" of machines ............ ro per cent;

Interest on capital .............. 5 per cent;

Price of one k.w.-hour of energy....... ro centimes (2 cents).

Let $I^{\prime}$ equal the resultant current at the generators when there is compensation; and let us assume that the resistance-loss in the generators, at full load, amounts to 4 per cent of the normal effective output.

Under these conditions, the saving in resistance-loss in the generators when there is compensation, will be (in francs)

$$
0.04 E_{1} I_{w}\left[\left(\frac{\mathrm{I}}{\cos \phi}\right)^{2}-\left(\frac{I^{\prime}}{I_{w}}\right)^{2}\right] \times \frac{0.10}{1000} \times h
$$

wherein $h$ is the total number of hours of operation of the installation.

The saving in interest and depreciation, for the machines, -will be given by the sum

$$
\begin{aligned}
p_{2} \frac{E_{1} I_{w}}{\cos \phi}[I & \left.-\frac{\left(I_{w}+i\right)^{2}+\left(I_{w} \tan \phi-j\right)^{2}}{\left(\frac{I_{w}}{\cos \phi}\right)^{2}}\right] \times(0.03+0.05)+ \\
& +\left\{p_{0} \frac{E_{0} I_{w}}{\cos \phi}\left[\mathrm{I}-\frac{\sqrt{\left(I_{w}+i\right)^{2}+\left(I_{w} \tan \phi-j\right)^{2}}}{\frac{J_{w}}{\cos \phi}}\right]-\right. \\
& \left.-p_{1} E_{2} I_{1}\right\}(0.10+0.05) . \ldots . . .
\end{aligned}
$$


Finally, the cost of the energy used by the synchronous motors will be (in francs)

$$
E_{1} i \times \frac{0.10}{1000} \times h . \quad . \quad . \quad . \quad . \quad .
$$

Numerical Example. Let us apply the preceding formulas to a particular case.

Assume a transmission of $500 \mathrm{k} . \mathrm{w}$. to a distance of 24.75 kilometers with 5000 volts at the receiving end, and a loss of ro per cent in the line.

Suppose it be desired to effect complete compensation by means of synchronous motors running without load. These motors must produce a reactive leading current

$$
j=I_{w} \tan \phi .
$$

Let us suppose, moreover, that the active current of the motors, when running without load, is

$$
i=0.10 I_{1} \text {. }
$$

Since we have

$$
I_{1}=\sqrt{i^{2}+j^{2}}=\sqrt{i^{2}+\left(I_{w} \tan \phi\right)^{2}},
$$

we find

and

$$
\begin{gathered}
i=\frac{I_{w} \tan \phi}{\sqrt{\mathrm{IOI}}}, \\
I_{1}=\frac{I_{w} \tan \phi}{\sqrt{0.99}} .
\end{gathered}
$$

Formula (28) gives

$$
p_{2}=436.36 \text { francs }(\$ 87.27) .
$$

The voltage at the receiving end being 5000 volts, and the loss in the line ro per cent, the initial voltage must be

$$
E_{0}=\frac{E_{1}}{0.9}=5550 \text { volts. }
$$

Let us assume the cost of the generators and motors to be

$$
p_{0}=p_{1}=100 \text { francs }(\$ 20) \text { per kilovolt-ampere. }
$$




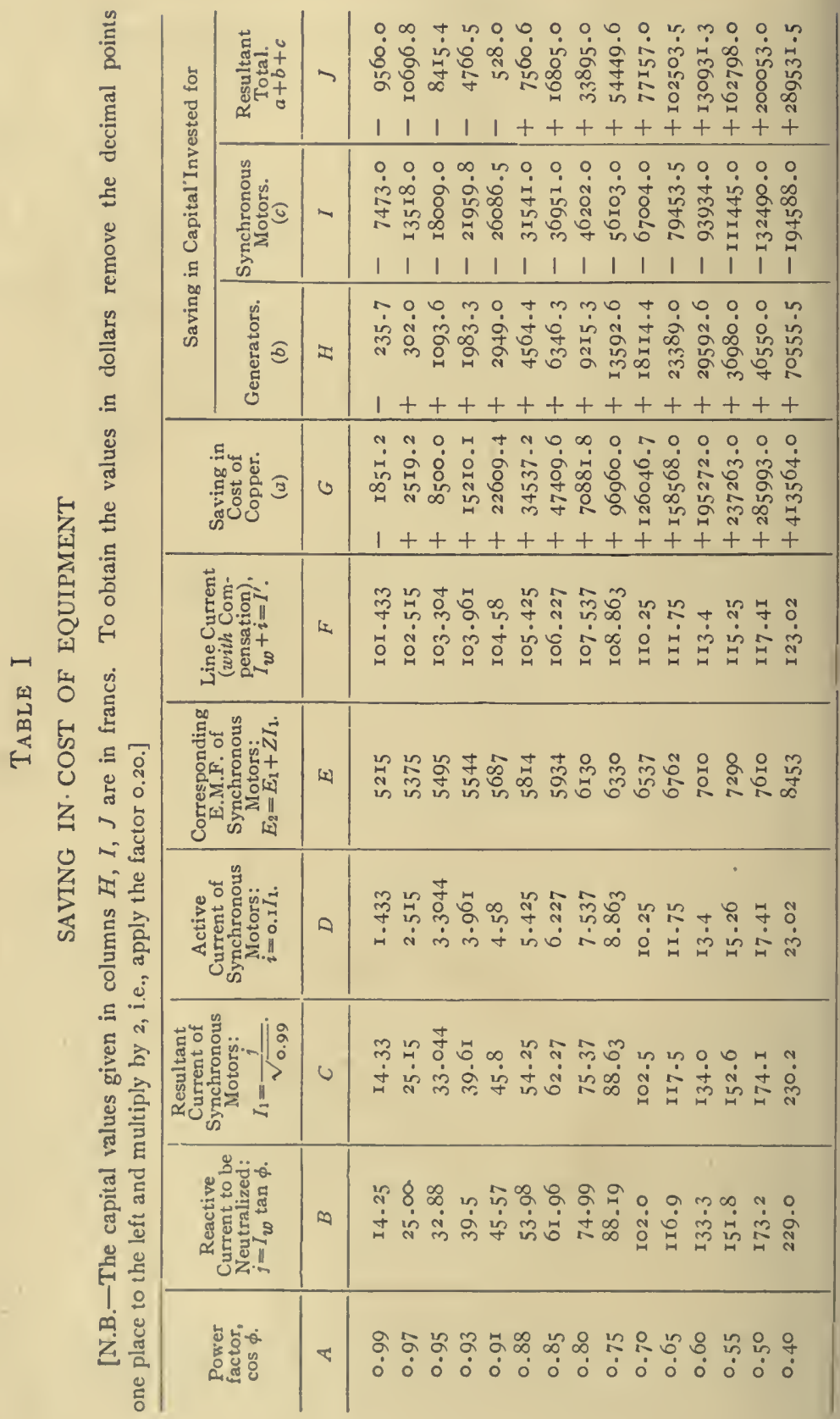




\section{DETAILED STUDY OF OPERATION WITH NORMAL LOAD 77}

\begin{tabular}{|c|c|c|c|c|c|c|}
\hline & & 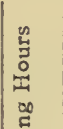 & & 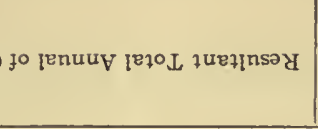 & $z$ & 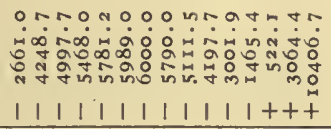 \\
\hline & & 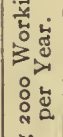 & $\begin{array}{l}\omega \\
0 \\
\text { के } \\
3\end{array}$ & 'sJO7ON snouOIYJUS U I & 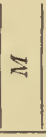 & 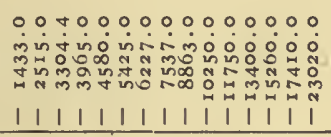 \\
\hline & & 点 & 蛋 & 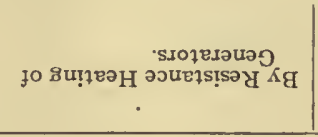 & 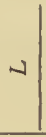 & 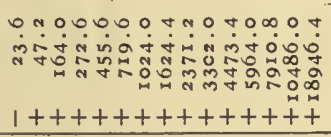 \\
\hline & 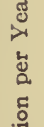 & క్ & & 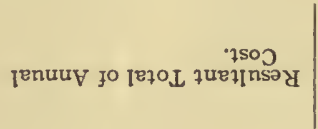 & N & 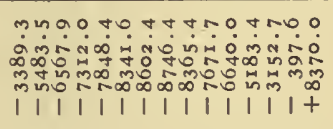 \\
\hline$\stackrel{\leftrightarrow}{0}$ & 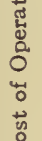 & 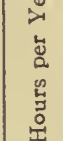 & 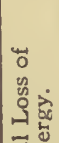 & 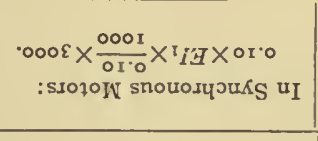 & $\rightarrow$ & 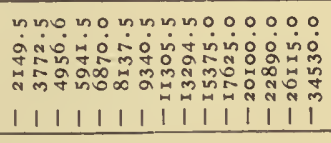 \\
\hline 岂 & $\underset{\Xi}{. \pm}$ & $\sum_{0}^{\infty}$ & 兘 & 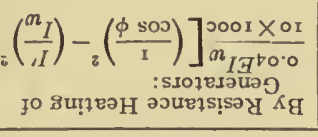 & - & 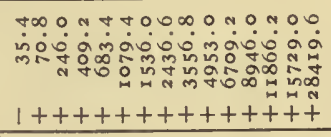 \\
\hline 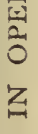 & & 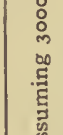 & 造 & 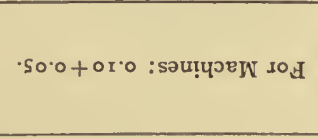 & 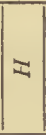 & 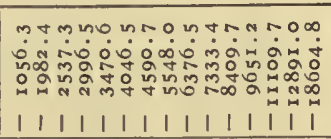 \\
\hline 岂 & & $\int_{4}^{0}$ & 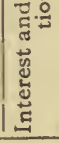 & 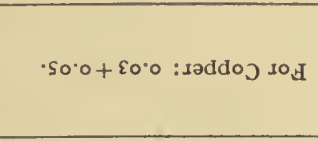 & $\circlearrowleft$ & 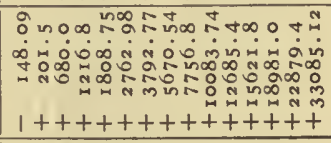 \\
\hline & & & : (uo & 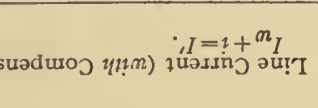 & 4 & 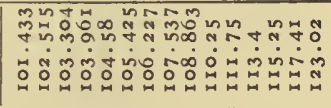 \\
\hline & & OPOIN & snouo. & 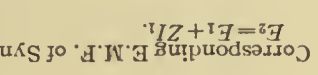 & $\Leftrightarrow$ & 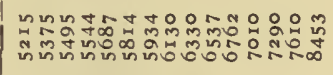 \\
\hline & & $: s+1$ & $270 \pi$ & 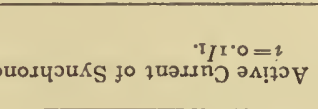 & a & 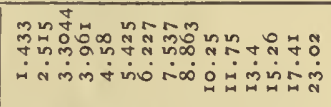 \\
\hline & & S.070 & IV sno & 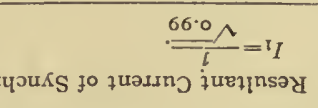 & $\cup$ & 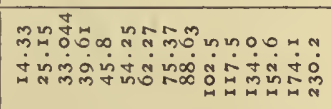 \\
\hline & & & :pəz! & $\begin{array}{c}\text { क } \phi \text { uеf } \\
m_{l=l}=l\end{array}$ & $\infty$ & 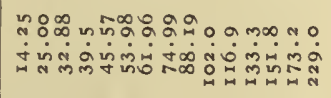 \\
\hline & & & & 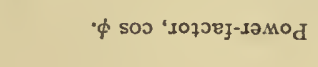 & 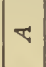 & 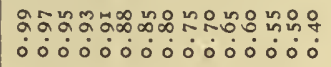 \\
\hline
\end{tabular}




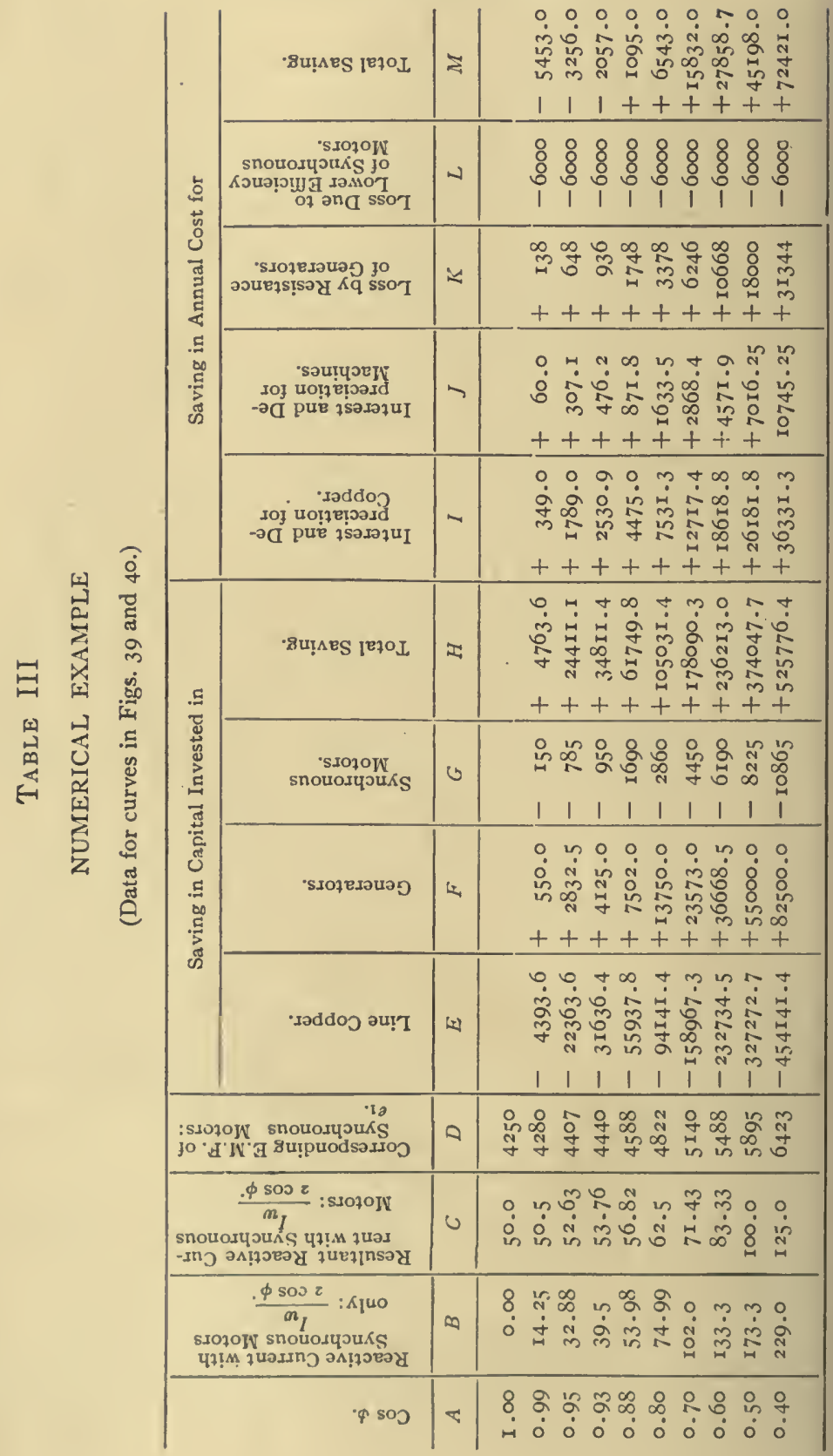


To estimate the size of the motors required, we will suppose them constructed for an E.M.F. equal to that which they should produce when run as compensating machines.

'For the sake of economy, the manufacturers tend more and more to increase the magnetic density, i.e., to "saturate" their machines, and it is scarcely prudent at present to count upon being able to increase very much their counter E.M.F. by increasing the excitation.

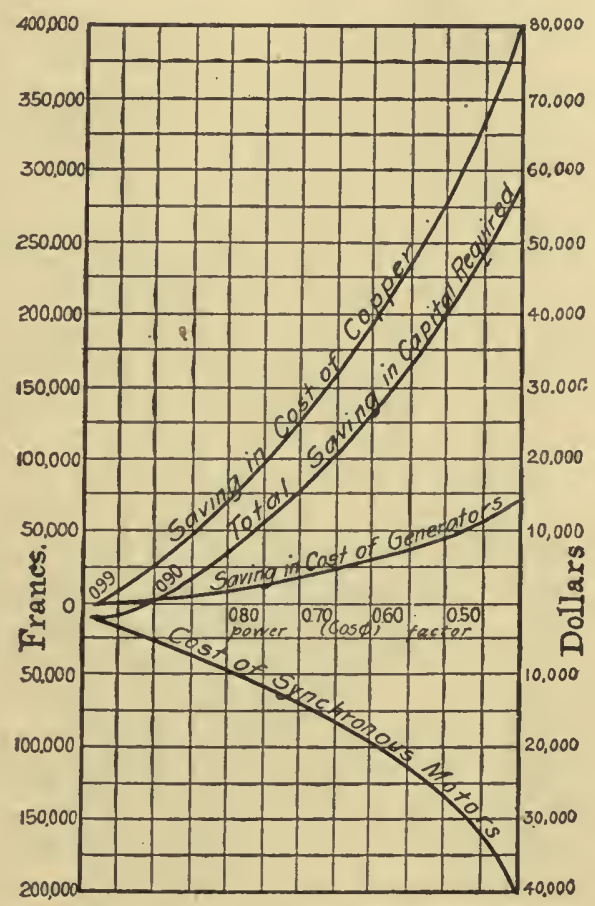

Fig. 37.

From the calculations corresponding to the values of $\cos \phi$ comprised between I and 0.40 , we obtain the curves given in Figs. 37 and 38. The curves in Fig. 37 give the saving in cost of equipment and the curves in Fig. 38 give the saving in annual expense of operation. It will be seen that if, from the standpoint of cost of equipment, the use of motors running without load becomes rapidly advantageous as $\cos \phi$ decreases, it is different, in any particular case, from the standpoint of the annual cost of operation. 
The depreciation of the machinery, which is higher than that of copper and also the consumption of energy required by the motors, (which latter is not compensated by the decrease in the losses of the generators), have the effect, together, of offsetting the saving made in copper, even through it may be somewhat important.

Economy of Compensation for the Distributing System by Means of Synchronous Motors Running with Load. There may be some

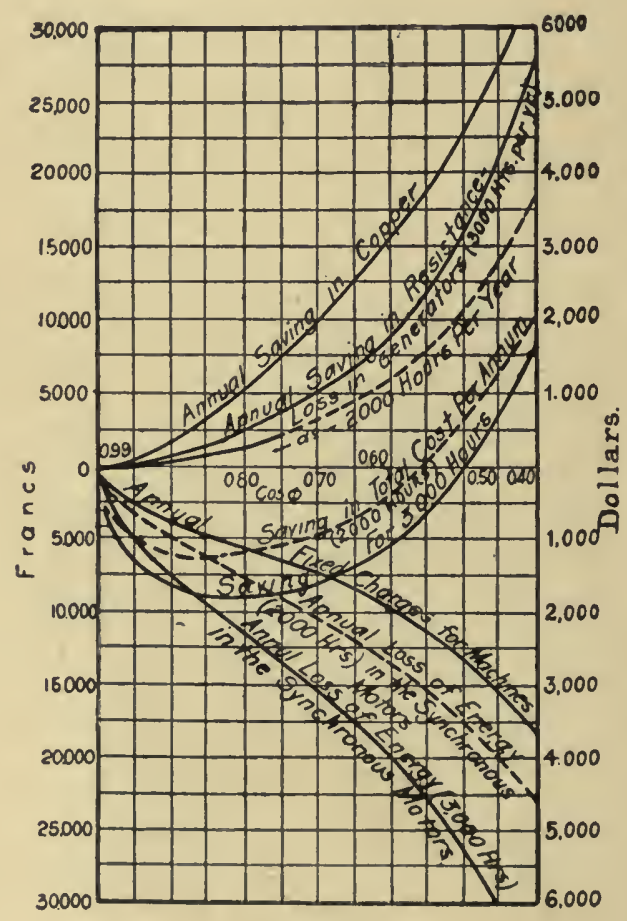

FIG. 38 .

hesitation in regard to the choice, in this case, because the efficiency of synchronous motors is generally lower than the efficiency of induction motors, and, besides, in order to produce a leading current, they must have a counter E.M.F. higher than that of induction-motors. The effect of this is to increase their rating and their cost. It is therefore better, in each particular case, to consider whether the use of synchronous motors is advantageous or not, from an economic point of view, due consideration being given to the necessities of the case. 
Suppose a long-distance power-transmission project in which it is possible to connect, with the distributing system, either some induction motors working with a certain lag, or else an equipment composed of one-half induction motors and one-half synchronous motors.

The line-current, with the induction motors alone, and with a phaseangle $\phi$, would be equal to $\frac{I_{w}}{\cos \phi}$. With half the capacity in synchronous motors added, and effecting complete compensation, the current would be equal to $I_{w}$.

It should be noted immediately that the loss by resistance-heating in the motors will be exactly the same in the two cases, because the magnetizing current of the synchronous motors has the same value as the reactive current of the induction-motors, it being, however, a current leading in phase.

Under these conditions, in order to maintain the loss of energy constant in the line, for different values of $\cos \phi$, the cost of linecopper, when theinduction-motors alone are used, should be

$$
p_{2} \frac{E_{1} I_{w}}{\cos \phi}
$$

and, with complete compensation by means of synchronous motors which carry half the load, the price of line-copper would be

$$
p_{2} E_{1} I_{w}
$$

For equal losses the cost of line-copper is proportional to the square of the current; the saving effected in the cost of copper, by introducing synchronous motors, would therefore be

or

$$
\begin{aligned}
& p_{2} \frac{E_{1} I_{w}}{\cos \phi}-\frac{p_{2} E_{1} I_{w}}{\cos \phi} \times \frac{I_{w^{2}}}{\frac{I_{w}^{2}}{\cos ^{2} \phi}} \\
& p_{2} E_{1} I_{w}\left(\frac{I}{\cos \phi}-\cos \phi\right) .
\end{aligned}
$$

The cost of generators, without compensation, would be

$$
\frac{p_{0} E_{0} I_{w}}{\cos \phi}
$$


and, with compensation, it would be

$$
p_{0} E_{0} I_{w}
$$

The saving in cost of generators, in the second case, would, therefore, be

$$
p_{0} E_{0} I_{w}\left(\frac{\mathrm{I}}{\cos \phi}-\mathrm{I}\right)
$$

Let $e$ equal the counter E.M.F. of synchronous motors operating with a magnetizing current $\frac{I_{w}}{2} \tan \phi$ which is lagging. Let $e_{1}$ equal their counter E.M.F. with the same magnetizing current leading in phase.

The values of $e$ and $e_{1}$ are deduced from formula (26) which gives

$$
e=E_{1} \sqrt{I+\left(\frac{Z I}{E_{1}}\right)^{2}+\frac{2 Z}{E_{1}}(j \sin \phi-i \cos \phi),}
$$

in which $E_{1}$ is the potential difference applied at the terminals of the motor and $I$ the resultant current.

The nominal power of the motors and, consequently, their cost, would be increased, in the second case, in the ratio $\frac{e_{1}}{e}$, and the increase in purchase-price of the synchronous motors, resulting from the introduction of compensation, and on the assumption that the price per $\mathrm{k}$.w. of the synchronous motors and induction-motors is equal, would be

$$
p_{1}\left(e_{1} \frac{I_{w}}{2 \cos \phi}-e \frac{I_{w}}{2 \cos \phi}\right)
$$

Let us see, now, what would be the saving in annual operating expenses:

The saving in interest and depreciation, for copper, is

$$
p_{2} E_{1} I_{w}\left(\frac{I}{\cos \phi}-\cos \phi\right)(0.03+0.05) \text {. }
$$

The saving in interest and depreciation, for the machines, is

$$
\left[p_{0} E_{0} I_{w}\left(\frac{\mathrm{I}}{\cos \phi}-\mathrm{I}\right)-p_{1}\left(e_{1} \frac{I_{w}}{2 \cos \phi}-e \frac{I_{w}}{2 \cos \phi}\right)\right](0.10+0.05) .
$$


The saving in loss by resistance-heating in the generators (which is proportional to the square of the current), is

$$
0.04 E_{1} I_{w}\left(\frac{\mathrm{I}}{\cos ^{2} \phi}-\mathrm{I}\right) \times h \text {. }
$$

Finally, we will assume the efficiency of the synchronous motors to be 8 per cent lower than that of induction-motors, which is rather

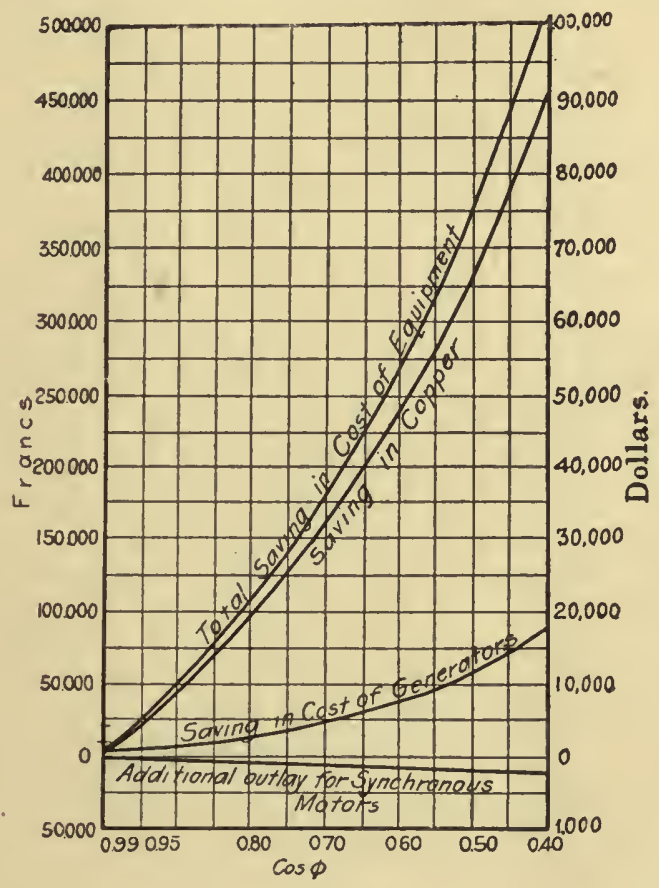

FIG. 39.

exaggerated, because synchronous motors are actually constructed whose efficiency approaches that of induction-motors. We will assume, however, that the efficiency is lower, in order to justify the assumption of equality of cost per k.w. for the two classes of motors.

The increase in annual cost of the energy expended in the synchronous motors, resulting from that difference in the efficiency, would be

$$
0.08 \times \frac{I_{w}}{2} E_{1} \times \frac{0.10}{1000} \times h
$$


in which, as before, $h$ is the total number of hours that the plant operates per annum.

Numerical Example. Let us take the same case of power-transmission already mentioned in which the total power to be transmitted $\mathrm{k} . \mathrm{w}$. is 500 the distance ro kilometers, and the potential difference, at the receiving point, 5,000 volts.

Let us assume that the total impedance of the synchronous motors

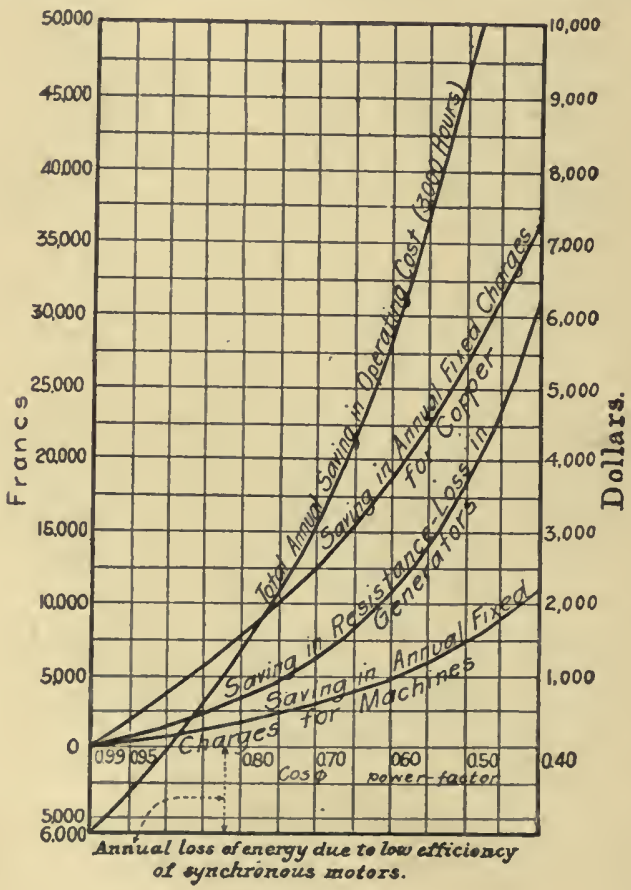

FIG̈. 40.

TABLE III

is $15 \mathrm{ohms}$, and that the plant is in operation a total of 3000 hours per annum. On substituting the corresponding numerical values in the preceding formulæ, and making the calculations we obtain data from which Table III (page 78 ) has been prepared, the results being shown graphically in Figs. 39 and 40.

The line parallel to the axis, in Fig. 40, represents the annual loss of energy due to the decrease of efficiency of the synchronous motors. 
It is seen that the curve of the saving which results from the use of synchronous motors working jointly with induction-motors, rises rapidly, and that it becomes positive for values of $\cos \phi$ which are all the greater the better the efficiency of the synchronous motors.

Regulation of Distribution-Voltage. The over-excitation of synchronous motors can, incidentally, enable the voltage to be raised

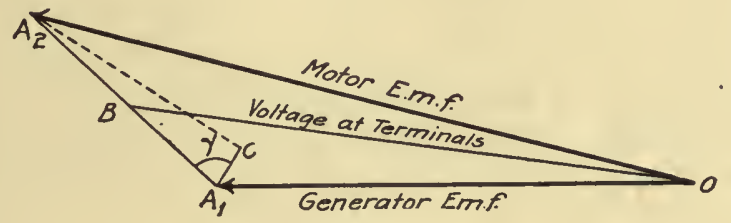

FIg. 4I.

at the terminals of motors and transformers connected with the line, and even at the terminals of the generator. The possibility of this is readily seen in the case where the line has a negligible resistance and reactance, and where there is only one generator and one motor which are both alike. The difference of potential at the terminals (Fig. 4r), is $O B$ ( $B$ being at the middle of $A_{1} A_{2}$ ), and by increasing

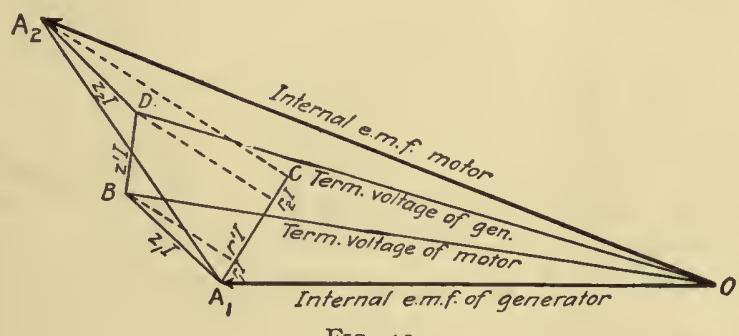

FIG. 42.

$O A_{2}$ this difference of potential can always be made as large as desired.

In the general case, by drawing, successively, with their phases, three vectors equal to the products of the current $\overline{A_{1} C}$ by the impedances of the generator, of the line, and of the motor, we obtain a broken line $A_{1} B D A_{2}$ (Fig. 42). The voltages at the terminals of the generator and of the motor are $O B$ and $O D$; and $A_{2}$ can always be sufficiently increased so as to make $O D$ and $O B$ greater than $O A_{1}$. 
Lahmeyer made an interesting application of this peculiarity at Bockenheim, where polyphase converters were operated like true synchronous motors. By increasing their excitation, the induced E.M.F. can be raised in such a way as to obtain a secondary voltage of distribution which is constant at all loads. The regulation can be made by hand or automatically. From this point of view rotary converters present a distinct advantage over stationary transformers, which introduce lags and whose secondary voltage necessarily decreases with the load.

(2) Compensation with Respect to the Generators. It is a twofold disadvantage, for the generators at the power station, to have the current out of phase, first, because the total current is increased

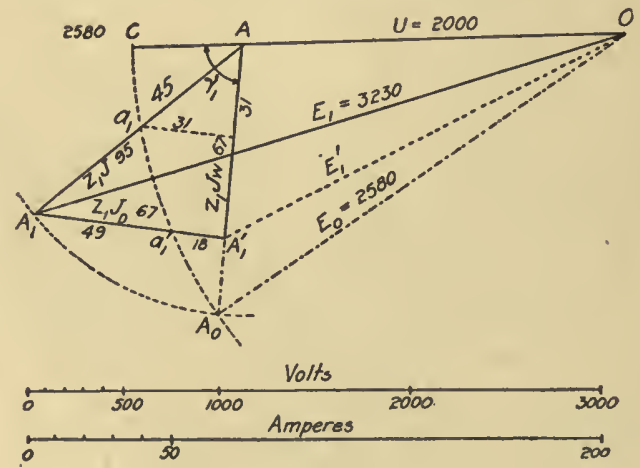

FIG. 43 .

and, secondly, because the armature-reaction is greater. To demonstrate this beyond question, let us consider a generator supplying a distribution-system at constant potential $U$. Let $J_{w}$ and $J_{d}$ be the active and reactive currents supplied to that system, $J$ being the resultant current. Let $R_{1}, X_{1}, Z_{1}$, respectively, be the resistance, reactance and impedance of the generator and of the feeders; and let them be considered constant, and as if located in a single apparains.

Let $\tan \gamma_{1}=\frac{X_{1}}{R_{1}}$ be the corresponding reactance-factor. Let us construct a diagram (Fig. 43) starting from the distribution-voltag? $U$, which is to be maintained constant at all loads.

The necessary E.M.F. $E_{2}$ will be obtained by compounding, with the voltage $\overline{O A}=U$, two vectors representing the volts absorbed by the impedance under the action of the two currents $J_{w}$ and $J_{d}$. The 


\section{DETAILED STUDY OF OPERATION WITH NORMAL LOAD 87}

first $\overline{A A_{1}{ }^{\prime}}=Z_{1} J_{w}$, makes, it is known, the angle $\gamma_{1}$ with the voltage $U$. The second, $\overline{A_{1} A_{1}{ }^{\prime}}=Z_{1} J_{d}$, will lag $\frac{\pi}{2}$ behind the first, since $J_{d}$ is lagging $\frac{\pi}{2}$ behind $J_{w}$. The resultant $A_{1} A$ corresponds to the real current

$$
J=\sqrt{J_{w^{2}+J_{d}^{2}}}, \quad \text {. . . . . . . . }
$$

and the line $O A_{1}$ represents the internal E.M.F. which the generator must produce in order that the distributing voltage shall equal $U$. The diagram gives the following formula, which is analogous to formula (26):

$$
E_{1}=U \sqrt{1+\left(\frac{Z_{1} J}{U}\right)^{2}+2 \frac{Z_{1}}{U}\left(J_{w} \cos \gamma_{1}+J^{d} \cdot \sin \gamma_{1}\right)} \ldots
$$

In this case $\gamma_{1}$ being always very large, $A_{1} A_{1}{ }^{\prime}$ is almost parallel to $U$. In general, we can write, approximately,

$$
E_{1}=\sqrt{\left(U+Z_{1} J_{d}\right)^{2}+\left(Z_{1} J_{w}\right)^{2}}
$$

It is seen that the reactive current requires a much greater increase of E.M.F. than the active current. If we had $J_{d}=0$, the alternator would develop the same power with a lower current, $\overline{A A_{1}{ }^{\prime}}$, and a lower E.M.F., $\overline{O A_{1}^{\prime}}$.

Let us suppose the current $J$ to be the maximum which the alternators can produce; and let us describe, around $A$ as a center, a circle of radius $\overline{A A_{1}}=Z_{1} J$. The manufacturer must have allowed a sufficient margin in the excitation to attain the E.M.F. $E_{0}$, which enables this current to be produced through a dead resistance while keeping the distribution-voltage at the value $U$; but if he confined himself to doing that, as was his right, it would be necessary, when the lag increases, to reduce the output in such manner that the point $A_{1}$ will remain on the circle $A_{0} C$, described from $O$ as a center, with $E_{0}$ as radius.

In such cases the effect of the reactive current becomes absolutely objectionable, and it is then very necessary to reduce it by employing means such as those just considered.

Fig. 43 can serve as a numerical example in the case of a $2000-$ volt distribution where the receiving apparatus has a mean powerfactor of 0.70 and the generator has an impedance of ${ }_{5} 5 \mathrm{ohms}$ (the 
two scales being therefore in the ratio of 15 to 1 ). We will now study this example in detail.

Numerical Example. Suppose a case where energy is to be distributed for mechanical purposes by means of a line supplying a great number of induction-motors. During the day, when a portion of these motors are running at light load, their mean power-factor is about 0.70 , i.e., the active current $J_{w}$ is approximately equal to the reactive current. Let us assume that it has been decided that the total power thus distributed will be about $100 \mathrm{kw}$.

The distribution-voltage is 2000 volts; the line-current has been predetermined on the assumption of a power-factor of 0.70 ; and, therefore, assuming an average efficiency of 0.75 , the current would be

$$
J=\frac{100000}{2000 \times 0.75 \times 0.70}=95 \text { amperes. }
$$

The line, having one ohm of resistance (its efficiency being about 95 per cent), and being supposed to have negligible inductance, gives a voltage-drop of 100 volts. An alternator has therefore been provided, of sufficient capacity to produce $95 \times 2100=200 \mathrm{k} . \mathrm{w}$., and it is believed that ample allowance has been made. This alternator will have, for example, $X_{1}=18 ; R_{1}=1.6$; whence $Z_{1}=13$ ohms, and $\tan \gamma_{1}=8.3$. The machine furnished by the manufacturer is regulated for a dead-resistance load and, consequently, it is capable of giving, with its fields almost saturated, the F.M.F. which seems necessary for the full load, by making $O A=U=2000$ volts, and $E_{0}=$ $O A_{0}=2580$ volts.

On putting the machine in operation an unexpected drop of voltage is noted, and it is impossible to obtain the 95 amperes calculated. In fact, if we draw the segment $A A_{1}^{\prime}$ at an angle of $45^{\circ}$, starting from $O A_{1}$, it is not 2580 volts which would be necessary, but practically,

$$
E_{1}=\overline{O A_{1}}=3230 \text { volts. }
$$

Since the machine can only produce 2580 volts, the load-point will have to be moved to the point of intersection, $a_{1}$, of the angleline $r$, with the circle $A_{0} C$. The machine cannot, therefore, deliver more than 45 amperes at the voltage of 2100 volts; and, since the corresponding active current is only 32 amperes, it will be seen that the alternator, although bought for $200 \mathrm{k}$.w. and expected to be good for roo k.w., can give, in actual service, only $2 \mathrm{I}+2100=46 \mathrm{k} . \mathrm{w}$.; and yet the manufacturer is not to blame. 
To overcome the difficulty, it will be sufficient to move the loadpoint to $a_{1}{ }^{\prime}$, because the power will be that needed (the active current being $A_{1}{ }^{\prime} a_{1}^{\prime}$ ) without requiring that the total current, $A a_{1}^{\prime}$, should materially exceed the predetermined current. This only requires that 49 amperes of reactive current should be eliminated from the 67 which exist. The most advantageous method of obtaining this result consists in replacing a few induction-motors by synchronous motors. If the change is made, for example, with a certain number of motors representing $0.75 \times 2100$ volts $\times 25$ amperes $=39.5 \mathrm{k} . \mathrm{w}$. of mechanical power, the reactive current suppressed is already 25 amperes; and it is sufficient, then, to produce an equal current in the opposite direction, by means of synchronous motors. If we assume the latter to have the same efficiency. they will also absorb 25 amperes of active current, so that their total current will be about 35 amperes (same as that of the induction-motors which they replace.

Their E.M.F., calculated, in the manner above indicated, with the object of producing the 25 amperes of reactive current, will be only 2660 volts, if a single motor of 35 amperes be used, and 2525 volts if two motors of $\mathrm{I} 7.5$ amperes each are used, etc. These voltages can be attained, as a rule, with motors bought for running at 2000 volts.

These motors will necessarily be working under imperfect conditions, since they will be only partly loaded. The installation, as a whole, will, therefore, be losing an output of $\frac{35-25}{25} \times 40=\mathrm{r} 6 \mathrm{k}$.w. But, on the other hand, it will be gaining, in the generator-output, $(67-3 \mathrm{I}) 2100=76 \mathrm{k} . \mathrm{w}$; hence there should be no hesitation in adopting this method.

The conditions taken, in this example, are not exaggerated, considering the apparatus which has been used until recently, and in which a drop of voltage of 30 per cent in the alternators under full load on a dead resistance was a frequent occurrence. These conditions occurred at Bockenheim, where, according to Lahmeyer, the output of the generators was doubled by introducing synchronous motors. These conditions would occur on many of the actual distribution systems if induction-motors were extensively introduced.

With the more recent types of alternators the conditions are better, and, consequently, the use of synchronous motors may be less advantageous. Nevertheless the reduction of generator-output by the use of synchronous motors can still, easily, amount to as much as 25 to 
30 per cent. For this reason it is always desirable, in any extensive installation, to consider the expediency of utilizing a sufficient number of synchronous motors, to avoid this reduction of output.

Comparison between Synchronous and Induction-Motors. The preceding remarks show the great advantages which synchronous motors present in comparison with induction-motors, in certain cases. Their stability of operation is not inferior to that of induction-motors, because a single-phase induction-motor, and even a polyphase induction-motor, also falls out of step when too much overloaded, unless the secondary resistance has been made quite large, at the expense of efficiency. The two points of inferiority of synchronous motors are, first, the fact that they are not self-starting, and, second, their low efficiency. In reality, their inferiority in these two respects is not as great as supposed, because it is possible to make synchronous motors which are self-starting without load, and as for their efficiency, it can be made as high as that of induction-motors, if they are properly designed.

On comparing a synchronous motor of Kapp type, with nonlaminated poles and expensive excitation, consuming, when running without load, a current equal to one-third the full-load current, with an induction-motor of the most perfect Oerlikon type (see article of Kolben, Elektrotechnische Zeitschrift, Nov. I, 1894) it is not difficult to establish the superiority of the latter. $\mathrm{Bu}$, if, in the said inductionmotor, the bars of the rotor were to be transformed into a D.C. exciting circuit, it would be observed that the difference in efficiency becomes very small.

Synchronous motors have a great advantage in construction because they admit of much higher magnetic densities than induction-motors.

[Use of Synchronous Motors to Raise Power-Factor in America. The compensating action of over-excited synchronous motors is utilized extensively in America for improving the power-factor of distribution-systems.

As an interesting example of the practical application of synchronous condensers some figures obtained from the Detroit Edison Company are given in Appendix C.

C. O. M.] 


\section{CHAPTER III}

\section{ADDITIONS TO THE THEORY. SECOND APPROXIMATION}

Imperfections of the Theory. Owing to the hypotheses on which it is based the preceding elementary theory, in common with all theories concerning alternating current machines, has certain imperfections. We shall discuss these briefly.

(I) When the currents are not sinusoidal, the results may be materially modified. At this point, it may be stated that we should precisely endeavor to make machines giving sinusoidal currents for power transmission, ${ }^{1}$ because, if their E.M.F. do not comprise exactly the same harmonics, the circuit becomes the seat of parasite currents, which are often very important. For example a certain Labour synchronous motor having a sinusoidal E.M.F. when run without load, being supplied with current from the distribution system of the Champs Elysees district, where the E.M.F. was non-sinusoidal, required a current almost double that which it consumed at the factory when supplied from an appropriate generator. This fact, among many others, shows that the effect of harmonics cannot, by any means, be considered negligible.

(2) The reactance $X$ of the motor is not constant, but varies with the excitation, with the strength of the armature-current, and with its phase-difference with respect to the E.M.F. $E_{2}$.

In practice, the angle $\gamma$ differs sufficiently from $90^{\circ}$ to make its variations, under the influence of the preceding effects, very small. Consequently, the line of zero phase-difference, $A_{1} N$, in Fig. 27 , is a curve which is always sufficiently flattened to warrant our continuing, without material error, to compare it to a straight line. But the power-

${ }^{1}$ This question was discussed by various authors in the Electrical World, which had the happy idea of opening its columns to that very interesting controversy. The greater portion of those who expressed their opinion, and among them the author of this work, were in accord in recognizing the important advantages of a sinusoidal E.M.F. from various points of view 
circles will be more or less deformed by these variations of reactance. The saturation of the fields, for strong excitation-currents, reduces these deformations and raises the right-hand branch of the $\mathrm{V}$-curves more rapidly toward the top than calculation would indicate. It is generally admitted that there is no other way of taking these deformations into account than by plotting the $\mathrm{V}$-curves experimentally.

(3) No attention has been paid to the variation of losses in the armature other than the resistance-loss. It may be remarked that these losses will vary but little, since the friction is substantially constant, and, in a motor supplied from a constant potential, the resultant E.M.F. in the motor remains almost constant. Likewise the induction and the hysteresis losses and the eddy currents remain approximately constant.

The only variable losses are those due to eddy currents in the fields, and the energy expended for excitation, neither of which have been taken into consideration in what precedes.

Practically, therefore, no great error has been made in supposing the losses in the iron to be constant while increasing slightly the noload losses (by way of compensation).

Attention is, therefore, called to this last source of imperfection merely to show its influence. In reality, the same thing might also be said in regard to the first two causes of imperfection, because the true object of this theory is only to elucidate the general course of the phenomena, and to give a method of calculation which will be sufficient for practical purposes. However, the effects to which attention is called above may assume sufficient importance, at times, to justify a somewhat more detailed investigation. This is more especially true in regard to variations of reactance.

Variations of Reactance with Lag of Current and Saturation of Fields. Armature-Reaction. The author has shown, elsewhere ( $L$ 'Indusirie Electrique, I899, p. 48I) that the self-induction of any alternator can be represented by two armature-reactions: 1

(I) An armature-reaction directly opposed to the exciting ampereturns and which is equivalent to

$$
\frac{1}{2} K N^{\prime} I_{d} \sqrt{2}=\frac{K N^{\prime} I_{d}}{\sqrt{2}}, .
$$

counter-ampere-turns. In this equation $I_{d}=$ the effective amperes of reactive current, with respect to $E_{2} ; N^{\prime}$ equals the number of arma. ture conductors in the induced field; and $K$ equals a coefficient which

'See Part II, Chapter I. 
depends on the shape of the pole-pieces. The induction-flux is produced by the algebraical resultant of the exciting ampere-turns and of these counter-ampere-turns.

(2) A transverse reaction producing a flux which closes itself transversely in the pole-pieces, without penetrating the field-windings and having a numerical value equal to

$$
\frac{L^{\prime} I_{w}}{\frac{1}{\sqrt{2}} N^{\prime}},
$$

wherein $L^{\prime}=$ a coefficient.

This resolution, of which the validity has been shown elsewhere, takes into account the lag, since the current intervenes by its two components (active and reactive) with respect to the E.M.F. of the motor. [The words "active" and "reactive" are here taken relatively to an E.M.F. induced with open circuit. The phase-angle thus obtained is therefore slightly different from that which would be found at the terminals.] It also takes into account the saturation of the fields, since the resultant flux of the fields is calculated from the ampere-turns themselves, by reference to an excitation-curve obtained experimentally.

As to the transverse flux, it is substantially independent of the saturation, because the reluctance of the magnetic circuit through which it finds its path is substantially constant. That is why $L^{\prime}$ can be considered constant.

With this assumption, it is easy to establish a new diagram for synchronous motors which will take these reactions into account. It will be sufficient to segregate the motor-reactances. Let $z$ and $x$ equal the impedance and the reactance, respectively, of the generator and of the line, and of the magnetic leakage of the motor; and let $R$ equal the resistance of the circuit, including the motor-resistance. (The generator-impedance may be neglected when the current is taken from a distribution-system.) Let $\varepsilon_{2}$ equal the effective motor E.M.F., i.e., the E.M.F. produced by the resultant fields of the field and armature ampere-turns.

The generator E.M.F. $E_{1}$ will be equal to the resultant of the following three components: First, the effective counter E.M.F., $A_{2} O=\varepsilon_{2}$, with its sign reversed; second, the transverse reaction-E.M.F. $A_{2} B=\omega L^{\prime} I_{w}$ with its sign reversed and having a phase-lead of $\frac{\pi}{2}$ ahead of $\varepsilon_{2}$; and, 
third, all the load-losses produced by the impedance $z$; this latter being represented by a vector $B A_{1}=z I$ (Fig. 44) which makes the angle $\phi$ with a line $B D$ that is itself drawn at an angle $\delta$ with $O A_{2}$ such that

$$
\tan \delta=\frac{\omega l}{R}
$$

The line $B A_{1}$ can serve to define the current $I$, in magnitude and in phase, if $B Y$ be taken as the axis of reference, with a scale $Z$ times greater than the scale of volts.

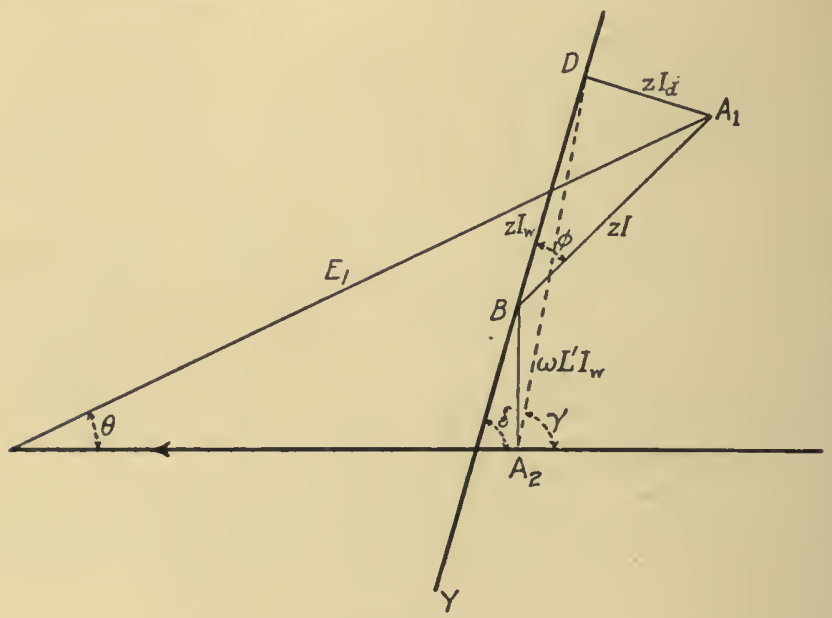

FIG. 44 .

This vector $z I$ can be resolved into two E.M.F.'s, viz.: $B D=z I_{w}$, produced by the active current, and $D A=z I_{d}$, produced by the reactive current.

The line $A_{2} D$, closing the triangle $A_{2} B D$, then represents the combination of the reaction-E.M.F.'s due to the active current, and it makes, with $O X$, the angle $\gamma$ defined by the equation

$$
\tan \gamma=\frac{\omega\left(L^{\prime}+l\right)}{R} .
$$

[It will be noted that the diagram differs from the preceding by the definitions of $z$ and $\delta$, which do not include the motor-reactance.]

Moreover, we will have

।

$$
\varepsilon_{2}=f\left(N i-\frac{\mathrm{I}}{\sqrt{2}} K N^{\prime} I_{d}\right)
$$


wherein $N i$ equals the exciting ampere-turns, and $f$ equals the function which defines the law of variation of the induced E.M.F. with the total inducing ampere-turns (this law being defined, in practice, by means of a curve). The ampere-turns of the armature are, in fact, demagnetizing, whenever $I_{d}$ lags behind $\varepsilon_{2}$, i.e., when the point $A_{1}$ is at the right of the axis $B Y$; and they are, on the contrary, magnetizing, when $A_{1}$ is at the left.

The segments $z I_{w}$ and $z I_{d}$ again measure, in magnitude and in phase, the active and reactive amperes by reference to some suitable scale; and the phase-angles (lags) are measured from the line $B Y$, which serves as axis of reference.

The output is the sum of the outputs corresponding to the currents $I_{w}$ and $I_{d^{0}}$ The induced E.M.F. is composed of the E.M.F. $\varepsilon_{2}$, produced by the direct field, and of the E.M.F. $\left(\omega L^{\prime} I_{w}\right)$, produced by the field of transverse reaction. The first is in phase with $I_{w}$ and the second with $I_{d}$. We thercfore have

$$
P_{2}=\varepsilon_{2} I_{w}-\omega L^{\prime} I_{w} I_{d}
$$

The diagram thereiore gives us again all the conditions of operation of the motor without material increase in complication. ${ }^{1}$

First Application of Corrected Diagram. Determination of Reactive Current as a Function" of the Excitation, with Constant Active Current. The preceding diagram enables us easily to find the values of the reactive current as a function of the excitation and of the active current, by the additional assistance of the excitation-curve for open circuit, either obtained experimentally or else predetermined by calculation. This curve (Fig. 45) gives, for each value, $F$, of the ampere-turns, the corresponding induced E.M.F.

${ }^{1}$ The problem is really much more complex, especially when the load changes suddenly, since the effect on the field-flux due to the armature-reaction does not then appear as fast as the change of armature-current. The flux may indeed remain constant for several seconds, even if the armature-current changes considerably. Hence, for sudden changes of load the phenomenon is largely governed by the true self-induction of the armature only. Thus, the armature-reactance may be only from I per cent to 3 per cent, whereas, for slowly changing loads, it would be equal to the synchronous reactance, say, 40 per cent. Therefore, machines which have good mutual induction between field and armature, such as high-speed motors and turbogenerators, may be unstable if the line-loss is great and if the load changes suddenly. The remedy in such cases is to install external reactances.-E. J. B. 
Let, for example, $I_{w}$ equal the given active current. Knowing the angle $\delta$ (Fig. 46 ), the segment $A_{2} B=\omega L^{\prime} I_{w}$ perpendicular to the

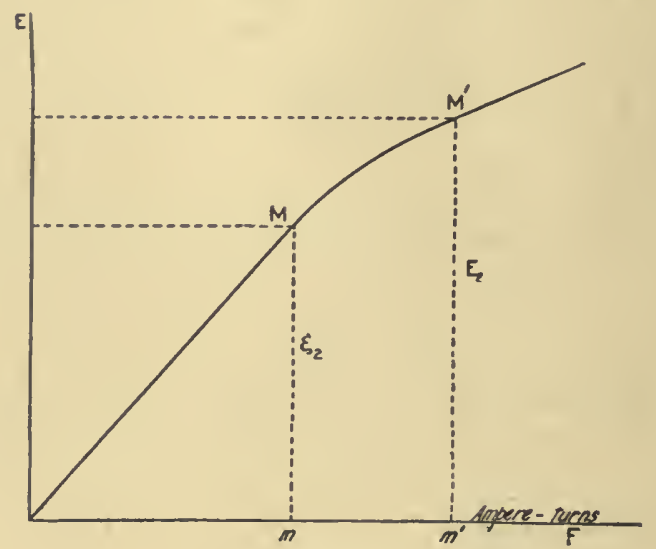

FIG. 45 .

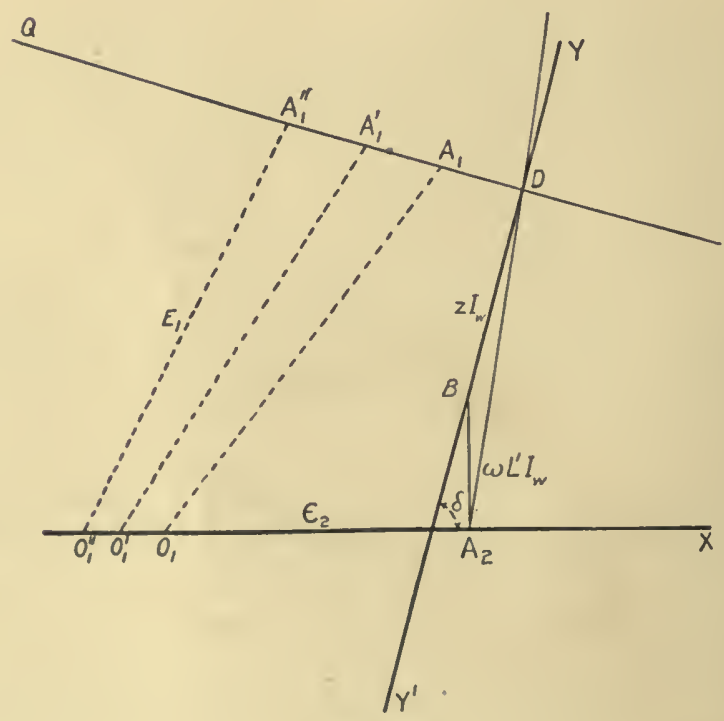

Fig. 46.

axis $A_{2} X$ can be drawn, and then the segment $B D=z I_{w}$ making the angle $\delta$ with $A_{2} X$ can be also drawn. 
We thus obtain the line of reference $B Y$, which represents the condition when the current is in phase with respect to $\varepsilon_{2}$. The locus of the characteristic point $A_{1}$ is a straight line $D Q$ perpendicular to $B D$, while the locus of the point $O_{1}$ is the straight line $O X$. For each value $I_{w}$, we therefore know the two straight lines at which the vector $A_{1} O_{1}$, equal to the supply E.M.F. $E_{1}$, should always terminate.

All loads corresponding to the valucs $I_{w}$ will therefore be determined by taking points such as $A_{1}, A_{1}{ }^{\prime}, A^{\prime \prime}{ }_{1}$, etc., on $D Q$, and by drawing circular arcs of radius $E_{1}$. The respective intersections $O_{1}, O_{1}{ }^{\prime}, O_{1}{ }^{\prime \prime}$, of these lines with $O_{1} A_{2}$, give some E.M.F.'s values, $\varepsilon_{2}$, which are proportional to $A_{2} O_{1}, A_{2} O_{1}{ }^{\prime}, A_{2} O_{1}{ }^{\prime \prime}$, while the corresponding reactive currents, $I_{d}$, are proportional, respectively, to the lengths $D A_{1}, D A_{1}{ }^{\prime}, D A_{1}^{\prime \prime}$.

For each pair of values of $I_{d}$ and $\varepsilon_{2}$, the corresponding exciting ampere-turns $F$ will then be obtained by referring to the excitationcurve. Let us take, on this curve, the point $M$, which has for its ordinate the resultant E.M.F. $\varepsilon_{2}$, thus determined. The corresponding abscissa, Om; measures the resultant ampere-turns.

$$
F \pm \frac{\mathrm{I}}{\sqrt{2}} K N^{\prime} I_{d}
$$

It is therefore sufficient to subtract, from this abscissa, the counterampere-turns of the armature, $=K N^{\prime} I_{d}$, in order to obtain the abscissa, $O m^{\prime}=F$, which represents the excitation, and, consequently, represents $E_{2}$, the E.M.F. induced on open circuit which would be obtained with this excitation.

It must not be forgotten that the reactive current $I_{d}$ is demagnetizing and positive if counted to the right of the axis $Y Y^{\prime}$, and magnetizing and negative if counted to the left (see page 94). The upper signs in the above formulæ apply in the first case, and the lower signs in the second case. We will therefore have $E_{2}<\varepsilon_{2}$ if the point $A_{1}$ is to the right of the point $D$ and, on the contrary, $E_{2}>\varepsilon_{2}$ if it is to the left.

In the particular case where a motor is connected directly, without additional rheostat or self-induction, on a constant potential circuit cf great output, and, when the impedance $z$ of its leakage is negligible, $D$ coincides with $B$, and the line $Q D$ then passes through the point $B$ itself; which simplifies the diagram still further.

The diagram which has just been explained is most precise in the case of an alternator working at the knee, or above the knee of the 
characteristic curve, or presenting uncqual coefficients of direct and transverse reactions. In the elementary theory it was assumed, on the contrary, that the permeability of the field-circuit is practically constant and that the two reaction-coefficients are equal.

Particular Case where the Permeability of the Field-Circuit is Constant and the Two Reaction-Coefficients are Equal. It is interesting to note how, in this case, the new and more general diagram comprises the previous forms as a particular solution. It is only necessary, indeed, to observe that the coefficient of transverse reaction, $L^{\prime}$, then

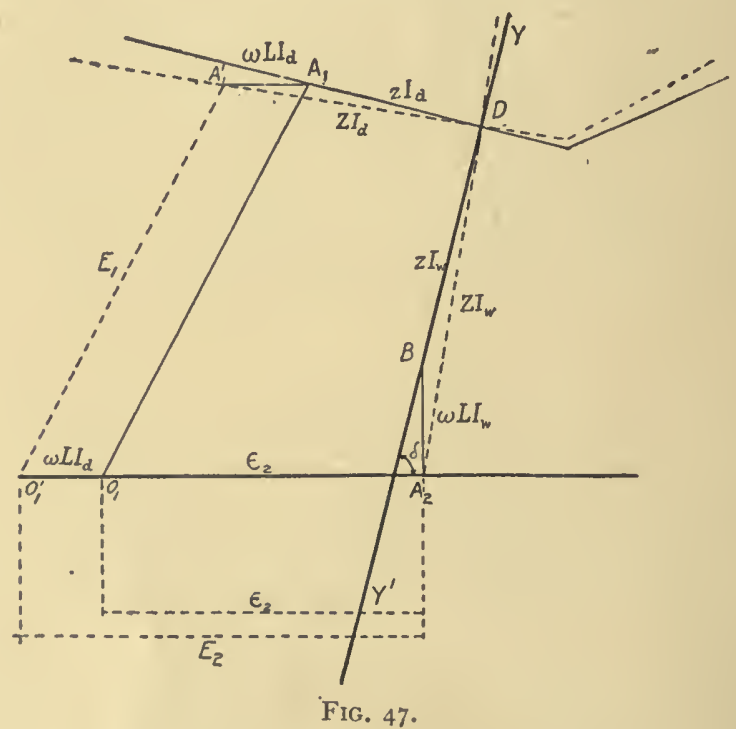

becomes equal to what we have termed the coefficient of mean selfinduction $L$. Likewise, the direct armature-reaction, which produces $\frac{\mathrm{I}}{\sqrt{2}} K N^{\prime} I_{d}$ counter-amperes-turns due to the reactive current, gives rise to a counter E.M.F. which can be represented by $\omega L I_{d}$. Therefore, between the effective E.M.F., $\varepsilon_{2}$, and the open circuit E.M.F. obtained with the same excitation, we have the relation

$$
E_{2}=\varepsilon_{2}-\omega L I_{d}
$$

$I_{d}$ being always taken as positive on the right-hand side of $B Y$ and as negative on the left-hand side. Suppose, for example, $I_{d}$ to be negative. 
It would be, necessary, in order to obtain $E_{2}$, to add to the segment $\mathrm{O}_{1} A_{2}$ (Fig. 47), a section

$$
O_{1} O_{1}^{\prime}=\omega L I_{d} \text {. }
$$

It amounts to the same thing if this segment is drawn parallel to and to the left of $A_{1}$; along $A_{1} A^{\prime}{ }_{1}$, and if a circular arc, of radius $E_{1}$, is drawn from $A_{2}^{\prime}$, to cut $O A_{2}$ precisely at the point $O^{\prime}$.

Now if we join $D A^{\prime}{ }_{1}$, it is seen that the triangles $D A_{1} A^{\prime}{ }_{1}$ and $D A_{2} B$ are "similar" triangles, since the side $D A_{1}$ and $D B$, also $A_{1} A_{1}^{\prime}$ and $B A_{2}$ are respectively perpendicular and proportional each to each. It may be deduced from this that the line $D A^{\prime}{ }_{1}$ is per-

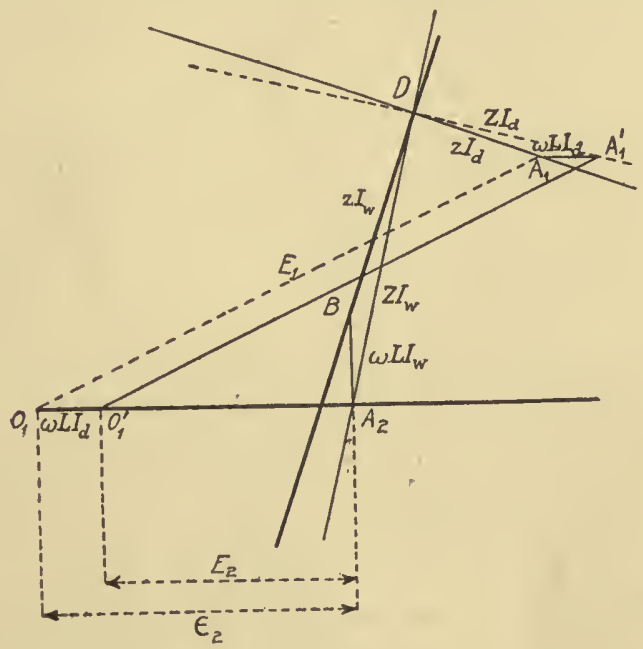

FIG. 48 .

pendicular to $D A_{2}$. On the other hand, the vectors $A_{2} D$ and $D A^{\prime}{ }_{1}$ being the resultants of the loss of potential in the motor and in the rest of the circuit, evidently represent $Z I_{w}$ and $Z I_{d}$, respectively, when $Z$ equals the total impedance of the circuit. The diagram composed of the right lines $A_{2} D, D A^{\prime}{ }_{1}, A_{1} A_{1}^{\prime}{ }_{1}, A_{2} O$, is, therefore, nothing more than the diagram which would have been obtained directly by the first method.

Q.E.D.

Fig. 48 shows the same analysis for the case where $I_{d}$ is lagging ( $I_{d}$ being positive). The precision of the power-formula already given can also be verified; for, in this formula,

$$
P_{2}=\varepsilon_{2} I_{w}-\omega L I_{w} I_{d},
$$


if we substitute for $\varepsilon_{2}$, its value,

we have

$$
\varepsilon_{2}=E_{2}+\omega L I_{d}
$$

as before.

$$
P_{2}=I_{2} I_{w}
$$

Second Application. Operation with Constant Excitation, on Constant-Potential-Supply System. When once the V-curves have been drawn it is very easy to obtain from them the different load-

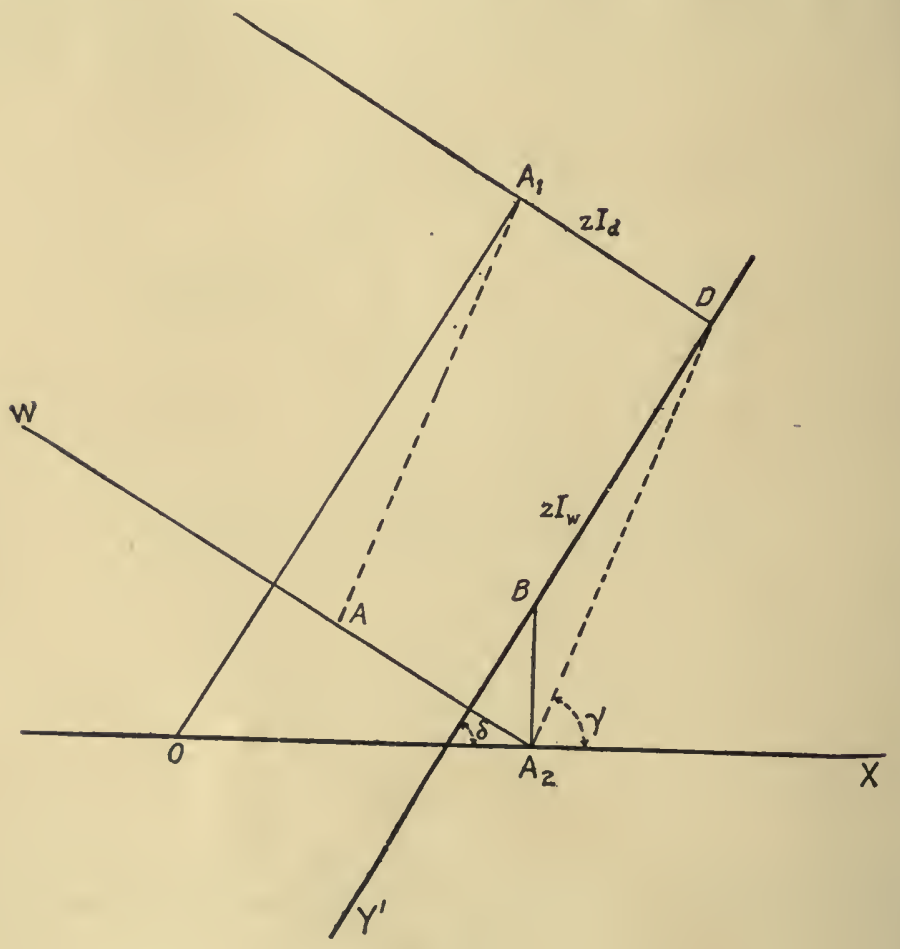

FIG. 49.

values corresponding to any given constant excitation. These values can also be determined directly from the diagram in the following manner:

Let us take the reactive current $I_{d}$ and let us find the active current $I_{w}$ corresponding to each value thereof. The direction $A_{2} D$ (Fig. 49) being, therefore, constant, can be drawn once for all. Likewise, the direction $A_{2} W$, to which the reactive segments $D A_{1}$ are parallel, makes 


\section{ADDITIONS TO THE THEORY. SECOND APPLICATION 101}

with $O A_{2}$ a known angle, $\frac{\pi}{2}-\delta$. Since we assume the values of $I_{d}$, we can calculate the values $z I_{d}$ and $\frac{I}{\sqrt{2}} K N^{\prime} I_{d}$. (As before $z$ represents the impedance of the armature only).

Let us draw, on $A_{2} W$, a segment $A_{2} A=z I_{d}$. The point $A$ must fall on a right line, $A A_{1}$, which passes through the point $A$ and which is parallel to $A_{2} D$. Again, knowing $E_{2}$, the excitation-curve, gives the excitation ampere-turns $F$. Subtracting $\frac{1}{2} K N^{\prime} I_{d}$, we have the resultant ampere-turns, and consequently, the effective E.M.F. $\varepsilon_{2}$. Let this be drawn on $A_{2} X$, to $A_{2} O$. The load-point $A_{1}$ must fall on a circle described around $O$ as a center with $E_{1}$ as radius. It is therefore determined by the intersection of that circle with the right line $A A_{1}$ previously drawn.

We thus obtain the value of $z I_{w}=B D$, and, consequently, the current $I_{w}$. From this we deduce the electric power applied to the motor,

$$
P_{2}=\varepsilon_{2} I_{w}-\omega L^{\prime} I_{w} \times I_{d}
$$

On the other hand, $I=\overline{\sqrt{ } I_{d}^{2}+I_{w}^{2}}$. We therefore obtain, by points, the relations between $I$ and $P_{2}$, both expressed as a function of $\varepsilon_{2}$ which serves as an intermediary variable.

V-Curves. The diagram does not lend itself to the direct determination of the $\mathrm{V}$-curves for constant power, owing to the complicated expression, which is not explicit as a function of $E_{2}$ and $I_{w}$, as it was in the elementary theory.

But these curves can be deduced from the curves of constant excitation, when these are drawn by the process indicated in the preceding paragraph.

Influence of Field-Saturation on Stability. The stability is evidently greater the higher the E.M.F. of the motor at the limit of lag. Now, for the same reactive current, the loss of potential produced by the direct reactance is all the less the higher the degree of excitation above the knee of the characteristic. It would therefore be advantageous, from the standpoint of stability alone, to have the fields saturated. But, there is a limit, here, owing to the amount of energy required for excitation, and the desirability of a certain margin to enable the voltage to be raised.

The cut-and-try process involved in finding the co-ordinates of the V-curves may be obviated, without constructing any curves, by 
utilizing Fig. 46. Knowing the value of $P$ and assigning a value to $I_{w}$, the equation,

$$
P_{2}=\varepsilon_{2} I_{w}-\omega L^{\prime} I_{w} I_{d}
$$

can be written as follows:

$$
\varepsilon_{2}-\omega L^{\prime} I_{d}=\frac{P_{2}}{I_{w}}=\mathrm{constant}=M .
$$

Taking, in Fig. 46, a point $A_{1}$ on the right line $D Q$, as a center, let a circle be described with a radius equal to $E_{1}$. . Let $O_{1}$ be the intersection of this circle with the right line $A_{2} X$. The values of $\varepsilon_{2}$ and $I_{d}$ can then be immediately deduced. We find

and

$$
\begin{aligned}
\varepsilon_{2} & =\overline{A_{1} O_{1}} \\
I_{d} & =\frac{i}{z} \bar{D} \overline{A_{1}} .
\end{aligned}
$$

These values introduced in equation (a) will give, for example,

$$
\varepsilon_{2}-\omega L^{\prime} I_{d}=N \text {. }
$$

Should this value be greater than $M$, it is because the point $A_{1}$ was located too near $D$. If, on the contrary, the value $N$ is less than $M$, it is because the point $A_{1}$ is too far from $D$. The point $A_{1}$ may be moved until the values found for $\varepsilon_{2}$ and $I_{d}$ give a value for $N$ equal to $M$. We can also proceed otherwise. Having found, quite easily, by the above method, two positions such as $A_{1}$ and $A_{1}^{\prime}$ which give, for the polynomial $N$, two values, one a little too large $(N)$ and the other a little too small $\left(N^{\prime \prime}\right)$, a point, $A_{1}{ }^{\prime}$, can be obtained by interpolation such that

$$
\frac{A_{1}{ }^{\prime} A_{1}}{A_{1}{ }^{\prime \prime} A_{1}{ }^{\prime}}=\frac{N-M}{M-N^{\prime \prime}}
$$

This gives the desired value of the power, $P$, with a sufficient degree of approximation for practical purposes.

Influence of the Wave-Form of E.M.F. The shape of the E.M.F. curves of the generator and motor should approach the sinusoidal form as much as possible in order to avoid instability and low efficiency. Let us first suppose that only one of the two machines departs from the sinusoidal form. By virtue of Fourier's theorem, its E.M.F. can be considered as the resultant of the superposition, upon a principal sinusoidal, of higher harmonics, i.e., of sinusoidals of higher frequency (odd multiples). 
The fundamental can alone produce useful power, because it is known that energy cannot be produced by the action of an E.M.F. until the current has the same frequency. Since the harmonic terms exist only in one of the machines they will simply produce parasite currents therein which will increase the heating by resistance-loss without any useful result.

It is known that the square of the effective current is equal to the sum of the squares of the effective currents of the component sinusoidals. The heating produced separately by each of the harmonics will therefore be added to the principal heating. This effect makes itself readily apparent in the $\mathrm{V}$-curves by an increase in the minimum current; which will be much greater than would be necessary to obtain the power actually developed. This can easily be observed by means of a wattmeter, which will indicate a real power no longer equal to, but really lower than, the apparent power (product of the effective current by the effective voltage). It cannot be possible that $\cos \phi$ is lower than unity, since the current is in phase, as we know, under these conditions.

It can even happen, as noted by Bedell and Ryan (Bedell and Ryan, Journal of Franklin Institute, Mar., I895, Fig. 9), on a motor having a strong third harmonic, that the V-curve breaks up into two branches, one of which is normal, at high excitations, the other being parasite, and very high at low excitations. Between these there is no stable load possible, without adding a strong self-induction in series. The author, who has himself observed similar phenemona (A. Blondel. Electrotechnische Zeitschrift, A pril 25, I895), attributes this favorable effect of a large self-induction (an effect which is contrary to the theory given on page 53) to the obliteration (throttling) of the harmonics, which meet, in passing through the self-induction, a reactance that is proportional to their own frequency.

In the case where it is the generator which has a distorted E.M.F., it can be eliminated, as M. Perot has shown (Perot, Comptes-Rendus, 6 Aug., I900, p. 337), by connecting in parallel, on the line supplied by this generator, a synchronous motor (or a converter) having a sinusoidal E.M.F. This apparatus, opposing no harmonic to those of the generator, short-circuits them without reducing the principal sinusoidal. If, therefore, we take $Z$ equal to the generator-impedance (or that of the group of generators) and $z$ equal to the motor-impedance, the amplitude of the harmonics, at the terminals, will be substantially reduced to $\frac{z}{Z+z}$ of their value on open circuit. Perot has 
thus been able, in a practical case, to reduce these harmonic terms to one-eighth of their value.

The case is different when the E.M.F.'s of the two machines are equally distorted, because the harmonics which produce the parasite current are then resultants of harmonics of the same order in the two machines. Since these harmonics are all of odd order, the two sets will oppose each other, if the principal sinusoidals are themselves opposed, as is approximately the case under normal operation when there is no lag.

Consequently, if like machines are used as generators and as motors, a considerably distorted E.M.F. curve would produce parasite effects which are much less important than in the first case, and almost negligible if care is taken to have the operation correspond to the lower part of the bend of the V-curve.

Simplified Diagrams. The diagrams shown in Figs. 44, 46, 49 correspond to the most general case where it is desired to take into account both the direct and the transverse armature-reactions.

Inasmuch as the direct armature-reaction alone affects the total excitation ampere-turns, it may seem, to some, an unnecessary complication to introduce the transverse reaction in the diagrams. For the benefit of those who prefer Kapp's simplified theory, other diagrams have been prepared (Figs. $44 a, 46 a, 49 a$ ) in which the transverse reaction is neglected, i.e., where it is assumed that $I_{u}=$ o.

In constructing these simplified diagrams it is important, as before, to determine the values of the resultant E.M.F. $\varepsilon_{2}$ by reference to the law of magnetic saturation as represented by the E.M.F. characteristic curve shown in Fig. 45a. In this diagram the abscissa represent excitation ampere-turns, and the ordinates represent E.M.F.'s. If the point $M^{\prime}$ represents the E.M.F. $E_{2}$ with open circuit, and if $O m^{\prime}$ represents the corresponding excitation ampere-turns, then, on subtracting from $\mathrm{Om}$ the ampere-turns corresponding to the reactive current $I_{d}$ or making $m m^{\prime}=\frac{K N I_{d} \text {, }}{\sqrt{2}}$ the ordinate $M m$ corresponding to the abcissa $O m$ will be the E.M.F. $\varepsilon_{2}$.

The impedance involved $(z)$ is that which corresponds to the magnetic leakage of the armature. Fig. $44 a$ shows how the corresponding potential difference $z I \cdot$ can be resolved into two components obtained by projecting $A_{1} A_{2}$ on $Y Y^{\prime}$ and on the line $D A_{1}$ perpendicular thereto.

The component $D A_{1}$ gives the value of $I_{d}$ when $z$ is known. 
ADDITIONS TO THE THEORY. SECOND APPLICATION 105

Fig. $46 a$ shows how the values necessary for constructing the $V$-curves are obtained; and Fig. $49 a$ represents the case where the synchronous motor is operating with constant excitation and at constant potential.

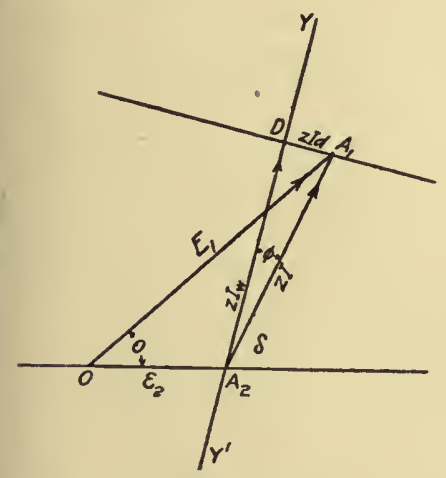

Frg. $44 a$.

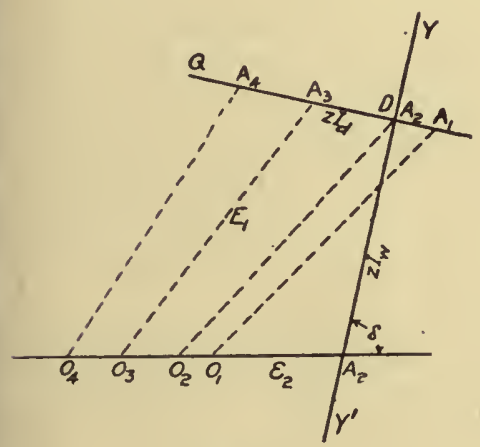

FrG. $46 a$.

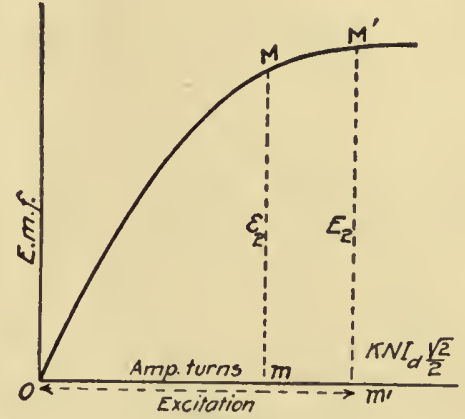

Frg. $45^{a}$.

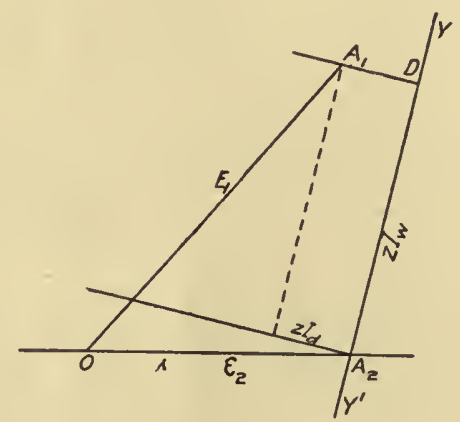

FIG. $49 a$.

These diagrams give a degree of precision which is generally sufficient for practical purposes, being intermediate between that obtainable with the simplified methods used in Chapter II and the more rigorous method used in Chapter III. Moreover, the diagrams $44 a, 46 a, 49 a$ express armature-reactions in terms of magnetomotive force more precisely than if the total $z I$ resultant were to be expressed by a magnetomotive force. 


\section{CHAPTER IV}

\section{OPERATION OF SYNCHRONOUS MOTORS. HUNTING}

Synchronous motors are not self-starting, the same as other electric motors, but they have to be started by some artificial means such as described hereinafter.

Starting by Direct Current. The simplest way, when there is a source of direct current and the synchronous motors are provided with a commutator for producing direct current for self-excitation, is to start the machine as if it were a D.C. motor, with the armature and fields connected in parallel. This plan can be especially adopted in transformer stations using motor-transformers, so as to start a set of motors operating dynamos as soon as one has been put in operation.

Starting with Alternating Current by Polyphase Motors. When there is no D.C. source, the alternating currents themselves must be used for starting the motor, either by mounting on the shaft of the machine a small induction-motor which serves to run it without load, or, more frequently, by using the machine itself as an induction-motor. (When a small motor is used its capacity should be about ro per cent of that of the machine. It can be even less, if it can withstand overloading for a few seconds.)

When using the machine itself as an induction-motor, the first step is to suppress the regular excitation, which would prevent the armature from insving. The second step is to connect the armature with the source of alternating supply, taking care to lower the voltage sufficiently to prevent excessive current through the armature. The armature then behaves as if it were the primairy winding of a revolving field machine in which the fields, and more especially the pole-pieces, play the role of secondary circuit. To obtain a torque equal to a quarter the normal torque, it usually requires, by this method, a current at least double the normal current. To complete this action, it is well to short-circuit the field-winding. Certain manufacturers have, for this purpose, arranged in the pole-pieces, as shown in Fig. $5 \circ$, a series of slot; containing windings or bars of copper, connected together 
like the armature of an induction-motor. In that case, the motor starts normally, and with a current which depends on the resistance. In any case this current is higher than in an induction-motor because the air-gap is greater and requires a stronger magnetizing current. For this reason, it is well to start the motors without load, in order to avoid excessive starting currents.

The production of the revolving field results from the eddy currents, but in the absence of these, the polepieces, even when laminated, give rise to hysteresis effects which are often sufficient for starting the motor without load. In certain motors having laminated poles the starting current is about double the normal full-load current.

This effect is explained by the fact that, during the rotation of the field, there is a stronger attraction in the direction of motion than in

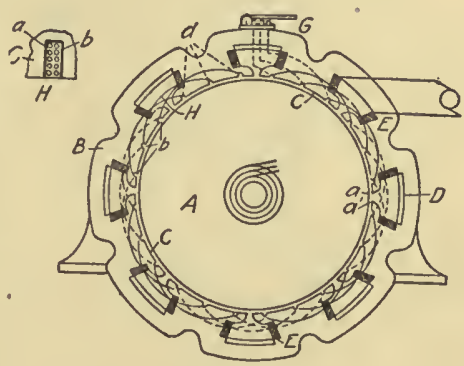

FIG. 50. the opposite direction owing to the lag of the magnetization behind the changes of field producing it.

Synchronism. In proportion as the armature-speed increases, the magnetic pulsations produced by it in the pole-pieces become less numerous, as can be seen on connecting a lamp to the field-circuit and noting its variations of brightness. It is well to provide a centrifugal regulator which connects this lamp in circuit only when the speed approaches synchronism, because, at lower speeds, it would be subjected to excessive voltage. When synchronism is almost attained(the speed generally remains slightly lower,)-the fields are excited when the phases come into opposition, the motor falls into step, and the current immediately diminishes, owing to the disappearance of the reactive current which was absorbed up to that time. The more carefully the time has been selected for closing the excitation-circuit, the more easily the motor will fall. into step. It is well, as a rule, to include, in the circuit, a variable self-inductance, which has the effect of preventing excess of current and of damping objectionable harmonics.

When once the motor is in synchronism, it can be loaded progressively, by shifting the belt from the idler to the driving pulley. This should be done very carefully when the mechanical resistance (load) 
In the particular case where it is possible to start the generator and the motor at the same time, the operation of starting becomes easier and there is no necessity of suppressing the excitation of the motor. The motor starts of itself by giving it a slight impulse, it falls into step at the very first period, owing to the low reactance at low speed, and its motion then accelerates synchronously with that of the generator, increasing gradually to full speed.

Observations on the E.M.F. Induced in the Poles. During the starting period, the fields are the seat of an important alternating flux whose frequency attains, at first, the same frequency as the alternating currents themselves. The result is the production of a very high E.M.F. in the field-winding, which is generally made of fine wire. If, therefore, this winding remains on open circuit, its insulation should be particulary good, and contact should not be made with its terminals. It is preferable to short-circuit this winding. This alternating E.M.F. naturally vanishes when synchronism is attained. It is obvious that the armature-brushes on the D.C. side must also be disconnected, since, otherwise, the pulsating current would produce a braking effect which would prevent the motor from starting.

Accessory Starting Apparatus. Installation of Synchronous Motors. The starting process involves the use of two kinds of accessory apparatus, i.e., phase-indicators and current-controllers.

It is also well to provide fuses and automatic circuit_breakers which break the main circuit whenever there is no excitation.

Phase-Indicators. Phase-indicators play the same role in this case as in coupling alternators in parallel. They are of the same kind and are connected in the same way. Their use is recommended for large-size motors.

When a synchronous motor is started by a direct current or by an auxiliary motor, a lamp of higher voltage than that of the supply-source may be connected in series with the motor. Owing to its high resistance, this lamp burns feebly at first under the action of the E.M.F. of the source of supply. In proportion as the motor increases in speed, if the field-winding is connected with a D.C. source, the E.M.F. which acts on the lamp, being the resultant of the generator and motor E.M.F.'s, will produce beats or pulsations which will gradually become less frequent. The proper time to connect the armature directly to the circuit is when the beats have become extremely slow, and when the lamp goes out, thereby indicating that the E.M.F.'s are in opposition.

When the motor is started without field-excitation, as an induction- 
motor, this method is no longer applicable and the phase-lamp (or else several lamps in series) must be connected to the terminals of the fieldcircuit, which is then subjected to the inductive action of the variable flux of the armature. The lamp burns brightly at first, then its bright. ness diminishes gradually, and the extinction of the lamp shows that synchronism is approaching; the direction which the E.M.F. will have when the circuit is closed is not determined, but this is generally of very slight importance.

With a well-constructed synchronous motor there is no need of a phaseindicator, because, when once it has attained its highest speed as an induction-motor (which may sometimes require several minutes), the powerful reactions which occur when the excitation-circuit is closed are sufficient to insure the machine falling into step.

Current-controllers. It is necessary, in order to reduce the amount of current taken from the line during the starting period, to employ a current-controller, owing to the absence of counter E.M.F. in the armature. Use may be made for this purpose of a simple rheostat or a choke-coil. Rheostats are more frequently used, on account of their simplicity. Switching appliances are used for the purpose of connecting them into circuit and then short-circuiting them, an armature circuit-breaker being interposed to break the circuit in case the field-excitation fails.

Fig. 5 I shows, for example, the diagram of the starting apparatus

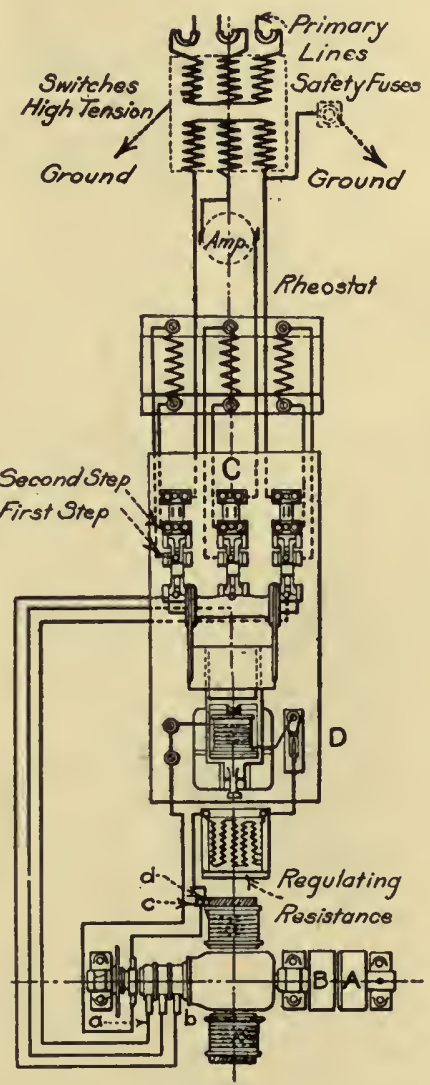

FIG. 5 I. of a certain type of three-phase motors ranging from I to 22 H.P. When the motor is at rest, the pulley is on the idler $A$, the triple-switch $C$ and the excitation circuit-breaker $D$ are open.

To start the motor the triple-pole switch $C$ is closed on the first steps, and the motor then receives a current from the supply-trans- 
former through a rheostat which limits the amount of current. The motor starts and attains synchronous speeu in from 20 to 30 seconds. The field-excitation circuit is then closed, and the counterweight of the circuit-breaker is lifted, which enables the triple-pole switch to be brought to the second position. The motor then receives the current

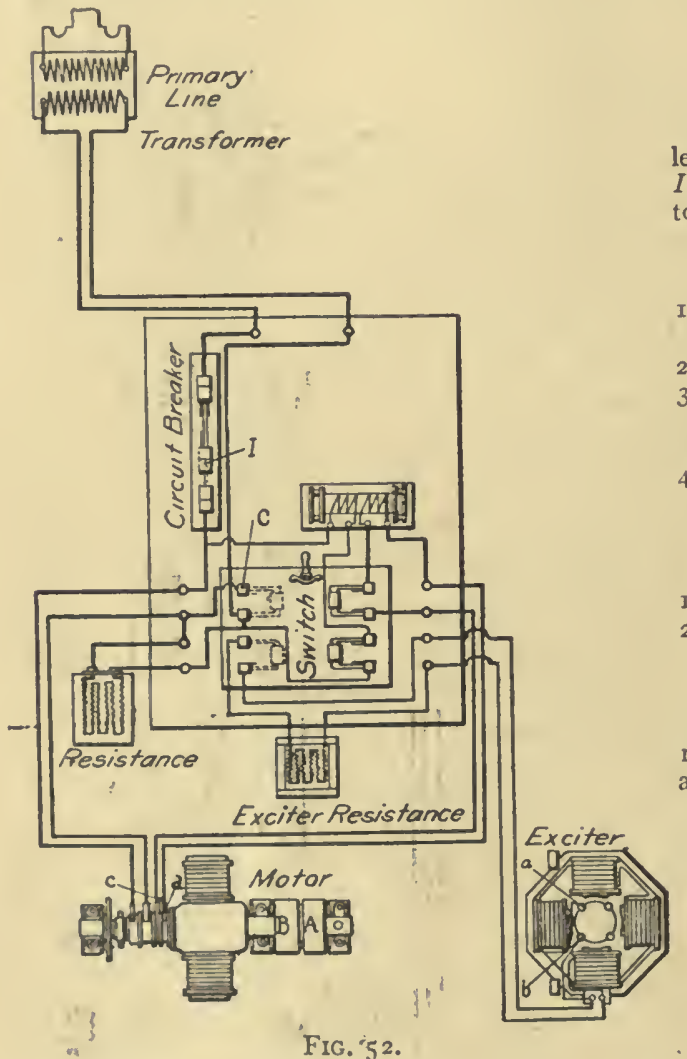

At Rest:

The belt is on the loose pulley $A$. The circuit-breaker $I$ is open. The switch $C$ is to the left.

\section{Starting:}

I. Throw switch $C$ to the right.

2. Close circuit-breaker $I$.

3. When synchronism is reached throw switch $C$ to the left.

4. Shift belt from pulley $A$ to pulley $B$.

\section{Stopping:}

I. Shift belt to pulley $A$.

2. Open circuit-breaker $I$.

$$
\text { N. B. }
$$

To change direction of rotation transpose wires $a$ and $b$, and $c$ and $d$. (Fig. 5r.)

directly, and it attains full speed. The belt can then be transferred gradually from the idler pulley to the driving pulley $B$.

To stop the motor we proceed in inverse order. The belt is shifted to the idler pulley $A$, the counterweight is dropped (which causes the three-pole switch to open), and finally, the circuit-breaker $D$ of the field-excitation circuit is opened.

For motors of 25 to 50 H.P. the single resistance is replaced by a variable rheostat and the switches have but one step. Once the speed is 
attained the rheostats are short-circuited. For these large motors, separate exciters are generally used, as already seen (Fig. 52).

The operation of starting without load does not require more than 20 to 30 seconds, and it is effected with a current not exceeding the normal full load current.
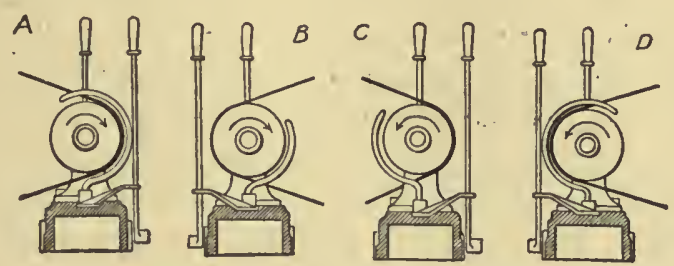

Fig. 53.

It will be observed that the process of starting involves the use of high-tension switches on the primary circuit, to isolate the transformer; also fuses on both the primary and secondary circuit.

The belt is shifted from the idler to the driving pulley in the ordinary way by a belt-shifter. "Fig. 53 shows the manner in which this shifter should be placed to correspond properly with the direction of rofation and the direction of driving, for an observer placed at the commutator end. Figures $A$ and $B$ refer to the case where the direction of rotation (indicated by the arrows) is the same as the hands of a watch; and Figs. $C$ and $D$ refer to the case where the rotation is in the opposite direction.

The use of a reactance-coil instead of a rheostat has the objection of increasing the lag of the current taken from the supply-source, which current already has too much lag. It is therefore preferable to replace it by an auto-transformer which enables the current of the motor to be increased at low voltage while

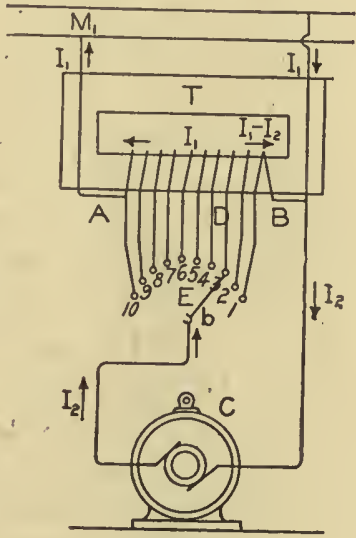

FIG. 54. reducing the amount of current taken from the line. Fig. 54 shows an example of this kind of arrangement which is in extensive use. The diagram represents the simplest case, i.e., that of a single-phase alternating current machine. It is a transformer with a single wind- 
ing having taps connected to contacts, over which moves the switch $b$. The two ends, $A, B$, of the winding are connected to the source of current-supply, while the motor is connected to the end $B$ and the switch $b$.

The winding operates like a potentiometer by which any fraction whatever of voltage can be obtained at will. At the beginning, very low voltage is obtained by placing the switch handle to the right-hand side. The handle is then moved gradually toward the left in proportion as the speed increases, and gradually raising the voltage applied to the motor, while reducing the current consumed by it. The current $I_{1}$ taken from the supply-source is a fraction of the current $I_{2}$, consumed by the motor, which is represented by the inverse ratio of voltages

$$
I_{1}=I_{2} \frac{E_{2}}{E_{1}}
$$

The winding of the transformer is traversed by the current $I_{1}$, between $A$ and $D$, and by the current $I_{2}-I_{1}$, between $D$ and $B$.

In the case of polyphase apparatus it is sufficient to install as many transformers with commutating switches as there are phases. All the levers of these switches are operated by one handle. An arrangement of this kind was utilized with polyphase current in the powertransmission installed for Messrs. Menier, at Noisiel in 1893 (PowerTransmission to Noisiel, Lumière Electrique, May, 20, 1894).

Starting of Single-Phase Machines. These machines can also be started by the first two methods, i.e., by means of an auxiliary D.C. motor, or by a small induction-motor which starts by means of an auxiliary phase such as, for example, "shaded pole" single-phase motors. It is desirable to be able to increase the speed of the machine gradually while keeping constant the speed of the small auxiliary motor, because the latter would be stalled if it were made to slow-up. To overcome this difficulty the motor is mounted on guides, and it transmits power to the larger machine by means of a friction-cone bearing against a large disk mounted on the shaft of the machine itself. The motor is gradually shifted on the guides in such manner that the friction-cone approaches the center of the disk until the moment when synchronism is attained.

The starting of the machine as an induction-motor is more difficult, but it can be accomplished by transforming temporarily the armature into a two-phase armature, by means of a second winding suitably 
provided for that purpose, and which can be of small wire, inasmuch as the time required for-starting is so short. By sending into this auxiliary winding a current which is more or less out of phase with respect to that in the principal winding, the armature produces a revolving field which is more or less imperfect and pulsating, but which is, nevertheless, sufficient to start the motor.

To produce the phase-difference between the two currents, recourse may be had to some one of the methods known for producing inductionmotors by means of a single alternating force. For example, use may be made of the Tesla method of introducing a self-induction into one branch of the circuit, and a resistance in the other, or use may be made of the Leblanc method, which is more perfect, and which consists in introducing a self-induction in one side and a capacity or a polarizer in the other.

But in order to reduce to a minimum the current taken from the line, there is again a great advantage in inserting a reducing transformer, built according to the principle already explained, and supplying current either for one phase or for both phases of the motor. One arrangement consists in supplying only the principal winding by means of the reducing transformer and in connecting the secondary winding directly to the switchboard bus-bars. In such a case, the secondary winding is made with a very high number of turns so as to have a very high self-induction and to absorb only a weak current having much lag with respect to the current of the other winding.

The arrangement employed by Labour (Fig. 52) is the reverse of this. In this case, it is the supplementary winding of the armature, made of fine wire, which is supplied by a reducing transformer, while the principal winding receives current direct from the switchboard through a rheostat. The double-throw switch $C$ being moved to the right (starting position) as shown in the diagram, the supplementary winding will then be in circuit with the secondary of the reducing transformer, and at the same time the rheostat is inserted in the principal circuit, which is closed by the circuit-breaker $I$. The motor starts as an induction-motor. When the speed approaches synchronism, the switch $C$ is suddenly thrown to the left; by this operation the current in the supplementary winding is broken, and the rheostat of the principal winding is short-circuited, at the same time that the fields are excited. The motor is then in step.

In large motors the circuit-breaker $I$ is replaced by a starting rheostat, the object being to avoid fluctuations in the lights supplied from the same 
distributing system. Sometimes an electromagnetic circuit-breaker is interposed in the excitation-circuit, its function being to open the main-circuit whenever the excitation-current fails.

The operation of starting and synchronizing requires about 30 seconds. The maximum current taken from the mains during this period does not exceed the normal current by more than 20 per cent; and it falls down to $\frac{1}{4}$ or $\frac{1}{5}$ the normal current when synchronism is attained.

Besides these general methods, there is still another, which can be used only in the case of motors having laminated field-poles. The method consists in sending alternating currents through the fieldcoils and through the armature-coils, which are then provided with a commutator. The latter can be adapted either to the principal winding, or to a supplementary winding, which serves only for starting and for excitation. This arrangement has been used by the Fort Wayne Company in the United States [Churchward, Eclairage Electrique, Vol. XVII, p. 4I]. At the time of starting, the current is sent through the armature and field-circuits connected in series; the machine then operates like a series-motor having a commutator; the direction of the current changes, both in the armature and in the field, and con-

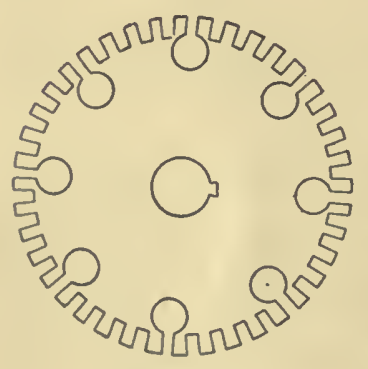

FIG. 55 . sequently the torque is always in the same direction and can thus cause the motor to start.

Both the field and armature of the Fort Wayne motor are laminated; the field has two windings, one being a fine wire winding for synchronous operation, the other being a coarse wire winding which serves for starting. The armature itself also has two circuits wound on a core composed of sheet-iron disks of the form indicated in Fig. 55. The principal winding, whicn serves for synchronous operation, is placed in the round holes, there being as many coils as there are field-poles, and the winding is connected with two collector-rings. The other winding, distributed in the slots, is similar to that of an ordinary D.C. drum winding with its commutator.

The operation of starting is accomplished by means of a single lever controlling the switches. If it is first placed in the position indicated in Fig. 56 the motor will be connected to the source of current 
like a series-motor, and it will start as such. When its speed attains synchronism, a centrifugal device closes the circuit with a third collector-ring on the right, causing an incandescent lamp to be lighted; the switch-lever is then moved, causing the normal connections to be made; the armature is then connected directly with the circuit; and the commutator circuit is connected with the fine wire excitation-circuit. The inductance in circuit is adjusted in such a manner as to permit the maximum allowable current to pass through the circuit when starting. This maximum current is about two to two and a half times the full load-current.

The efficiency of this type of motor is 70 per cent for a $2 \frac{1}{2}$ H.P. motor, and 80 to 85 per cent for motors of Io to I 5 H.P. They start readily under load, without excessive sparking at the brushes, the latter being of carbon and having a fixed position. The power-factor, which is very high when starting, does not fall below 0.95 to I under load.

For small motors, of I to 5 H.P., the Gramme commutator just mentioned can be replaced by a less complicated commutator having only one segment per pole of the motor. The small Ganz

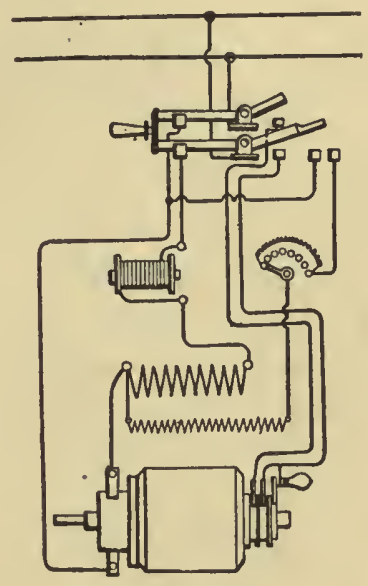

FIG. 56. motors are of this kind. Their fields are excited by commutated currents obtained from a transformer connected in shunt to the line. The even segments are connected to one terminal of the field-circuit, and the odd segments to the other. To start the motor, which can only be done without load, only one set of brushes need be used on the commutator, the others being put off. The current thus changes direction in the field-coils and in the armature coils, as already mentioned; but there is much sparking at the brushes during the whole time that the motor is starting. When the synchronous speed has been attained, which requires at least a minute, the brushes previously removed are again put on, by hand or else by a centrifugal regulator, or they may be put in circuit by means of a switch; sparking is then reduced to an allowable amount. Fig. 57 gives the diagram of the arrangement actually used (Blathy system). The field and armature windings are in parallel. The armature-current, coming from the transformer at roo volts, 
through the wires $I, I I$, enters into the armature by the terminal $A$, leaves by the terminal $B$ and passes through the secondary-winding $S_{1}, S_{2}$ of the transformer $E$, called compensator, which plays the role of self-inductance for throwing the armature-current out of phase.

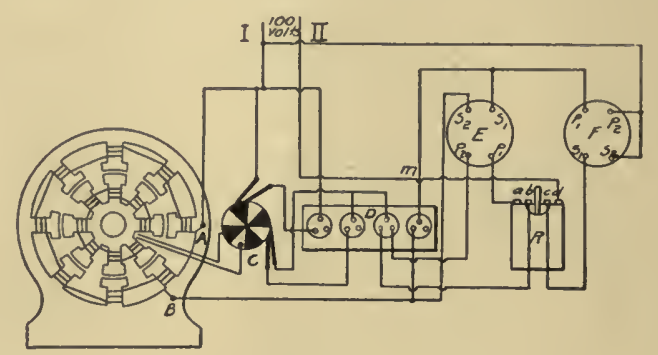

Fig. 57 .

The field-circuits are wound for a low voltage (25 volts) supplied by the secondary circuit $S_{1}, S_{2}$ of another transformer $F$.

At the time of starting, to overcome the induction of the fieldcircuit, the potential-difference at its terminals is raised by the primary winding of the compensating transformer $E$, whose secondary has the

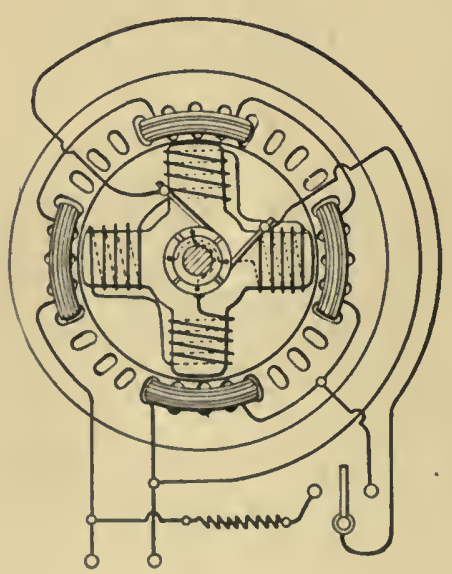

F1G. 58 . armature-current passing through it, as already stated. The field-current also passes through a fixed resistance, $a b$, in the rheostat, $R$, the object of which is to reduce the lag in the field-circuit, and to put it in phase with the armature-current.

The motor starts. When it attains synchronism, the current in the field is commutated; the connections are then changed by means of the double-throw switch $D$, which shortcircuits the secondary of $E$, connects

- the two neighboring brushes together and sends the secondary current of $F$ into the variable resistance $c d$ before it reaches the field-coils.

A motor of the same kind was put on the market some years ,ago by the firm of Brown-Boveri (Fig. 58). The motor starts by simply coupling in parallel the armature and the field-circuit and a resistance 
which is in series with it. To bring the phases of the two currents into proper relation, the brushes are moved an angle equal to $\frac{\pi}{2}$ during the operation of starting. When synchronism is attained, the brushes are brought back to their place, and the excitation-circuit is connected in shunt to one coil alone of the armature, so as to reduce the voltage.

Theory of Initial Synchronizing. Two cases must be considered, depending on whether the field, when separately excited, maintains its normal value independently of the speed, or whether the field is produced by means of commutated currents.

Separate Excitation. Let us again suppose the motor to be connected to a constant potential source $E_{1}$ and let us retain the same symbols as before. Let us designate by $T_{2}$ the duration of the period of the motor, which will differ from $T_{1}$ the period of the supply-current, so long as synchronism is not attained. For simplicity, let us write

$$
\frac{2 \pi}{T_{1}}=\omega_{1}, \quad \frac{2 \pi}{T_{2}}=\omega_{2}
$$

The equation of the current proceeding from the source of supply toward the motor is

$$
r_{2} i+l_{2} \frac{d i}{d t}=E_{1} \sin \omega t-\frac{\omega_{2}}{\omega_{1}} E_{2} \sin \left(\omega_{2} t-\theta\right),
$$

where $E_{2}$ equals the E.M.F. induced at the speed of synchronism, and $\theta$ represents, as before, the lag in phase of the motor. From this, neglecting the exponentials which occur in the integral, but which always neutralize each other quickly, and taking

we have

$$
\tan \phi=\frac{\omega_{1} l}{r}, \quad \tan \psi=\frac{\omega_{2} l}{r},
$$

$$
i=\frac{E_{1} \sin \left(\omega t_{1}-\phi\right)}{\sqrt{r^{2}+\omega_{1}^{2} l^{2}}}-\frac{\omega_{2}}{\omega_{1}} \frac{E_{2} \sin \left(\omega_{2} t-\theta-\psi\right)}{\sqrt{r^{2}+\omega_{2}^{2 / 2}}} .
$$

The power supplied to the motor at each instant is

$$
e_{2} i=\frac{\omega_{2}}{\omega_{1}} E_{2} \sin \left(\omega_{2} t-\theta\right)\left[\frac{E_{1} \sin \left(\omega_{1} t-\phi\right)}{\sqrt{r^{2}+\omega_{1}^{2} l^{2}}}-\frac{\omega_{2}}{\omega_{1}} \frac{E_{2} \sin \left(\omega_{2} t-\theta-\psi\right)}{\sqrt{r^{2}+\omega_{2}^{2} l^{2}}}\right] .
$$


Integrating between $t_{0}$ and $t$, the work done is

$$
\begin{aligned}
T=\frac{E_{1} E_{2}}{\sqrt{r^{2}+\omega_{1}^{2} l^{2}}} \frac{\omega_{2}}{\omega_{1}} \int_{t_{0}}^{t} \sin \left(\omega_{2} t-\theta\right) \sin \left(\omega_{1} t-\psi\right) d t \\
\qquad-\frac{E_{2}{ }^{2}}{\sqrt{r^{2}+\omega_{2}^{2} l^{2}}} \frac{\omega_{2}^{2}}{\omega_{1}^{2}} \int_{t_{0}}^{t} \sin \left(\omega_{2} t-\theta\right) \sin \left(\omega_{2} t-\theta-\psi\right) d t .
\end{aligned}
$$

While the second term increases indefinitely, the first is doubly periodical and has the form

$$
\int \sin \omega_{1} x \sin \omega_{2} x d x
$$

It is known that an integral of this form, taken within limits which comprise a whole number of periods of $\omega_{1} x$ and of $\omega_{2} x$, is equal to zero, so long as $\omega_{1}$ is different from $\omega_{2}$. If $\omega_{1}$ and $\omega_{2}$ are very different from each other, their zeros are very near each other and the amplitude is very small. On the contrary when $\omega_{2}$ is very near $\omega_{1}$ the periodicity of the integral increases in length to the point of becoming infinite.

Two cases are therefore to be considered:

I) So long as the speed of rotation is low, the first term is negligible in comparison with the second, provided that the inertia in the motor is sufficient and that only negative work is done. We then have

$$
\begin{aligned}
T & =-\frac{\mathrm{I}}{2}\left(\frac{\omega_{2}}{\omega_{1}} E_{2}\right)^{2} \frac{\left(t-t_{0}\right)}{\sqrt{r^{2}+\omega_{2}^{2} l_{2}^{2}}} \cos \psi \\
& =-\frac{\mathrm{I}}{2}\left(\frac{\omega_{2}}{\omega_{1}} E_{2}\right)^{2} \frac{r\left(t-t_{0}\right)}{r^{2}+\omega_{2}^{2} l^{2}} .
\end{aligned}
$$

It is therefore necessary to apply mechanical power to turn the alternator and with $\Omega_{2}=$ the speed corresponding to the period $I_{2}$, and $\Omega_{1}=$ the speed corresponding to the condition $\left(\Omega_{2}=\Omega_{1} \frac{\omega_{2}}{\omega_{1}}\right)$, the motor torque $C_{\omega}$ will have the following expression:

$$
\begin{aligned}
C_{\omega} & =-\frac{\mathrm{I}}{2}\left(\frac{\omega_{2}}{\omega_{1}} E_{2}\right)^{2} \frac{r}{r^{2}+\omega_{2}^{2} l^{2}} \frac{\mathrm{I}}{Q_{2}} \\
& =\frac{\mathrm{I}}{2} \frac{\omega_{2}}{\omega_{1}} \frac{E_{2}^{2}}{\varrho_{1}} \frac{r}{r^{2}+\omega_{2}^{2} l^{2}} \\
& =-\frac{E_{2}^{2}}{{ }^{2} \Omega_{1} r} \frac{\frac{\omega_{2}}{\omega_{1}}}{\mathrm{I}+\left(\frac{\omega_{2}}{\omega_{1}}\right)^{2}\left(\frac{\omega_{1} l}{r}\right)^{2}} .
\end{aligned}
$$


Taking $x=\frac{\omega_{2}}{\omega_{1}}$ we will have

$$
C_{\omega}=-\frac{E_{2}^{2}}{2 \Omega_{1} r} \frac{x}{\mathrm{I}+x^{2} \tan ^{2} \phi} .
$$

The curves in Fig. 59 represent $C_{\omega}$ as a function of $x$, when the constant $\frac{E_{2}^{2}}{2 \Omega_{1} r}$ is taken as unity, and when $\tan \phi$ is given values equal to 5 and ro.

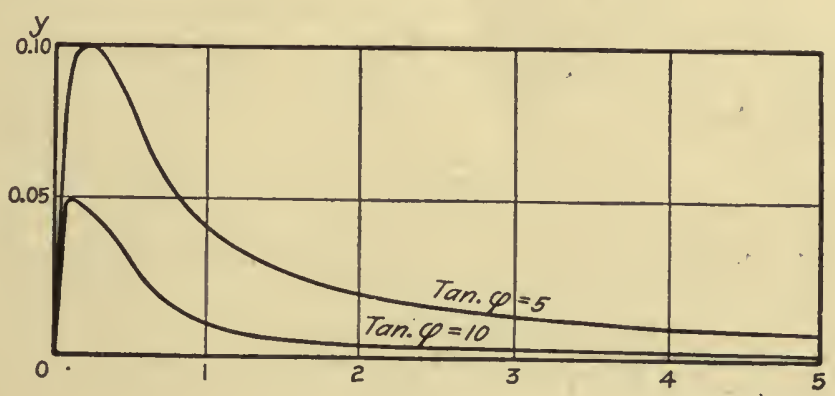

FIG. 59.

It is seen that the torque $C_{\omega}$ increases rapidly, at first, with the speed, and then decreases more slowly with a maximum

$$
x=\frac{\mathrm{I}}{\tan \phi} ; \quad C_{\omega}=-\frac{E_{2}^{2}}{2 \Omega_{1} r} \frac{\mathrm{I}}{2 \tan \phi}=-\frac{E_{2}^{2}}{4 \Omega_{1} \omega_{1} l} .
$$

To bring back the motor to a speed near. that of synchronism, it would be necessary to exert a mechanical force (torque) near that which corresponds to $x=\mathrm{I}$, or

$$
C=-\frac{E_{2}}{2 Q_{1} r} \frac{\mathrm{I}}{\mathrm{I}+\tan ^{2} \phi}
$$

i.e., the same as would be required to make the alternator rotate when short-circuited upon itself.

2) When once $\omega_{2}$ is near $\omega_{1}$, the first term is no longer negligible. The integral can no longer be evaluated except by supposing $\omega_{2}$ to be constant within the limits of integration, i.e., during a great number of periods. 
Then, taking $t_{0}$ as the origin, $\left(t_{0}=0\right)$, we will have

$$
\begin{aligned}
\int_{0}^{t} \omega_{2} \sin \left(\omega_{2} t-\theta\right) \sin \left(\omega_{1} t-\phi\right) d t \\
=\frac{\omega_{2}}{2} \int_{0}^{t} \cos \left[\left(\omega_{1}-\omega_{2}\right) t+\theta-\phi\right] t t \\
\quad-\frac{\omega_{2}}{2} \int_{0}^{t} \cos \left[\left(\omega_{1}+\omega_{2}\right) t--(\theta+\phi) d t\right. \\
=\frac{I}{4} \frac{\omega_{2}}{\omega_{1}-\omega_{2}} \sin \left[\left(\omega_{1}-\omega_{2}\right) t-(\phi-\theta)\right] \\
\quad-\frac{I}{4} \frac{\omega_{2}}{\omega_{1}+\omega_{2}} \sin \left[\left(\omega_{1}+\omega_{2}\right) t-(\phi+\theta)\right] .
\end{aligned}
$$

Noting that

$$
\frac{\omega_{2}}{\omega_{1} \Omega_{2}}=\frac{\mathrm{r}}{\Omega_{1}}
$$

the total value for the torque, at each instant, would therefore be

$$
\begin{aligned}
C= & \frac{E_{1} \hat{E}_{2}}{\Omega_{1} \sqrt{r+\omega_{1}^{2} l_{2}^{2}}}\left\{\frac{\mathrm{I}}{4\left(\omega_{1}-\omega_{2}\right)} \sin \left[\left(\omega_{1}-\omega_{2}\right) t-(\phi-\theta)\right]\right. \\
& \left.\quad-\frac{\mathrm{I}}{4\left(\omega_{1}+\omega_{2}\right)} \sin \left[\left(\omega+\omega_{2}\right) t-(\phi+\theta)\right]\right\}-\frac{E_{2}^{2}}{2 \Omega_{1}} \frac{\frac{\omega_{2}}{\omega_{1}} r}{r^{2}+\omega_{2}^{2} l^{2}}-C_{\iota_{0} .}
\end{aligned}
$$

When $\omega_{1}$ is very near $\omega_{2}$ the amplitude of the first term becomes very high, and this is the case so long as $\omega_{1}$ is not exactly equal to $\omega_{2}$. At that moment, there is a discontinuity; and the term in question vanishes.

Under these conditions, the inertia is low compared with the enormous torque which is developed, and the motion of the alternator can undergo pulsating variations of speed which are considerable. The hypothesis which forms the basis of our reasoning concerning the nature of the motion is no longer fulfilled and the equations no longer have any value.

Only one conclusion, therefore, can be drawn, i.e., it is necessary to bring the motor, by some process, to a speed at least 'equal to that of synchronism, before connecting it with the source of current-supply.

A second method of starting a motor without load, and which can be used with success every time there is only one power-transmission, consists in starting simultaneously the generator and the motor. A very weak impulse then suffices to make $\omega_{1}=\omega_{2}$, at the moment of 
starting. The two machines therefore fall readily into step, and if the resisting couple due to passive mechanical resistances (air-friction, bearing-friction, hysteresis, etc.) is not too high, the generator drags the motor, in its progressive acceleration, the two always remaining in synchronism.

Field Due to a Commutated Current. In this case, the effect produced is altogether different. Two periods must again be distinguished, i.e., whether $\omega_{2}$ is very different from $\omega_{1}$, or whether, on the contrary, it is of the same order of magnitude.

In the first case, i.e. at the beginning of the operation of starting, the current is reversed a great number of times, both in the armature and in the fields, while the armature is moving an angular distance corresponding to a period $T_{2}$. The motor behaves like a commutatormotor, and produces a torque which is always in the same direction and which gradually increases the speed.

In the neighborhood of the normal speed, the effect of the commutator on the excitation becomes apparent. The alternations of flux are commutated in a dissymmetrical manner, with the consequence that a very powerful field with slow oscillations is produced, on which pulsatory undulations that are more feeble are superposed. This effect may be expressed by the formula,

$$
\Phi=\Phi_{0} \cos \omega t_{0}
$$

in which $t_{0}$ has a value which is variable as a function of the time.

As soon as the two periods $T_{1}$ and $T_{2}$ are near each other, the small undulations disappear before the large undulation, namely, the undulation of $t_{0}$. The frequency, $\frac{\mathrm{r}}{T}$, of this great oscillation is evidently equal to the least common multiple of $\frac{\mathrm{I}}{T_{1}}$ and $\frac{\mathrm{I}}{T_{2}}$. The period, $T^{\prime}$, therefore, lengthens indefinitely, when $T_{2}$ approaches $T_{1}$, at the same time that the value of the field thus produced increases.

Under these conditions, the power furnished by the motor varies according to a complicated function. The oscillations of the field can be taken into account on replacing $\omega t_{0}$ by $\omega^{\prime} t$ and substituting the value

$$
E_{2}=-\frac{d \Phi}{d t}=-\omega^{\prime} \Phi_{0} \sin \left(\omega^{\prime} t-\zeta\right)
$$


in the expression $e_{2} i$. But the equations are then no longer rigorous and can only be used to predict the occurrence, in the neighborhood of synchronous speed, of heavy fluctuations resulting in alternate accelerations and retardations which are very marked and for which the inertia of the system cannot compensate. As soon as one of these accelerations becomes strong enough, it.will, at one bound, bring the speed to its normal value, leaving the motor synchronized.

The starting of motors of the Ganz type may thus be explained approximately, without our being able to take exactly into account the effects of inertia. It is known, moreover, that synchronous motors do not always run at synchronous speed, but at a speed near it, which is periodically variable, owing to the production 'of the alternating field already mentioned. It is an extreme case of oscillations of load, which we now proceed to discuss.

Oscillations of Synchronous Motors. It is easily observable, by means of a dead-beat ampere-meter connected in the armature-circuit, that the operation of a synchronous motor is always accompanied by fluctuations above and below the normal current, which are due to oscillations above and below the ..synchronous speed. With a good motor, operating in a satisfactory manner, these oscillations are rapid and slight, except when the load undergoes sudden variations. But, with certain motors, these oscillations attain a considerable amplitude and they are complicated by slow variations of very high amplitude, giving rise to exaggerated current-values like those due to a shortcircuit, which usually end by the motor falling out of step;: it may even be that synchronous operation is altogether impossible.

There are here two distinct phenomena, which will be examined separately, i.e., short-period oscillations of the motor itself and longperiod parasite oscillations due generally to foreign causes.

Short-Period Oscillations. Any variation of the speed of the generator or any sudden variation of the resisting effort at the motor-shaft gives rise to an oscillating condition of motor-speed. If the generator, for example, has, taken the lead, the driving torque becomes stronger than the resisting torque, and it causes the armature to accelerate gradually; but, owing to inertia, the acceleration lasts longer than would be necessary to attain the corresponding phase at the condition of equilibrium, and the motor, in its turn, takes the lead in phase. From that moment, as its driving torque diminishes, the excess of the resisting torque tends to produce a retardation; the motor slackens in speed, and its phase is soon behind. what it should be; and so on. 
Consequently, oscillations of speed occur around the normal speed value, the effects being altogether analogous to the oscillations occurring in the speed of steam engines which are provided with imperfect governors.

The characteristic feature of the short-period oscillations of the motor-speed is that this oscillatory movement occurs without loss of synchronism, in other words, each acceleration in one direction or the other is stopped by an opposing torque which limits the amplitude, as is the case with the oscillations of a pendulum.

This torque is easily determined by the variation of power of the two machines (A. Blondel, "Coupling of Alternators," La Lumière Eletrique, Vol. XLV, p. 352). Let us first suppose these two machines to be similar; let $P_{1}$ and $P_{2}$ represent, respectively, the generatorpower and the motor-power. The variation in the electric power received by the motor and transmitted by the generator, per unit of angle $\alpha$ of angular variation, taking ${ }_{2} P$ to equal the number of poles, is, according to the formulæ on page 38 , as follows :

$$
\begin{aligned}
\left(\frac{d P_{1}}{d \alpha}-\frac{d P_{2}}{d \alpha}\right) & =p\left(\frac{d P_{1}}{d \theta}-\frac{d P_{2}}{d \theta}\right) \\
& =p \frac{E_{1} E_{2}}{Z}[\sin (\gamma-\theta)+\sin (\gamma+\theta)] \\
& =\frac{2 p E_{1} E_{2}}{Z}(\sin \gamma \cos \theta) .
\end{aligned}
$$

In this expression $Z$ equals the total impedance, and $\gamma$ is, as before, the angle of lag between the current and the resultant E.M.F., the relations being such that

$$
\tan \gamma=\frac{\omega L}{R} .
$$

The correcting torque is obtained by dividing this power by the mean angular velocity

$$
\frac{\omega}{p}
$$

From this we have

$$
C=\frac{p^{2}}{\omega}\left[\frac{2 E_{1} E_{2} \sin \gamma \cos 0}{Z}\right] .
$$


If we let $K$ equal the inertia of the motor, the period of oscillation produced by this torque may be deduced by the known formula,

$$
\theta=2 \pi \sqrt{\frac{K}{C}}
$$

whence, on substituting, and noting that $\frac{2 E_{2}}{Z}$ is nothing more than the short-circuit current, $I_{s c}$, of one of the machines-the motor-closed on itself, there remains

$$
\theta=\frac{2 \pi}{p} \sqrt{\frac{K \omega}{E_{1} I_{R c} \sin \gamma \cos \theta}}
$$

or, if we suppose $\theta$ to be small enough so that $\cos \theta$ may be taken as equal to unity, we will have

$$
\theta=\frac{2 \pi}{p} \sqrt{\frac{K \omega}{E I_{s c} \sin \gamma}}
$$

In the case where the machines are not similar and are running at different speeds $\alpha_{1}$ and $\alpha_{2}$, it can be shown, in the same way ( $A$. Blondel, loc. cit., p. 357), that the equation of oscillatory movement is

$$
\omega \frac{d^{2} \theta}{d t^{2}}+\frac{\left(P_{1}\right)_{\theta}-\left(P_{1}\right)_{\theta_{0}}}{\left(\frac{K_{1}}{p_{1}^{2}}\right)}-\frac{\left(P_{2}\right)_{\theta}-\left(P_{2}\right)_{\theta_{0}}}{\left(\frac{K_{2}}{p_{2}^{2}}\right)}=0 .
$$

In the case of small oscillations, if we replace the numerators by their values, we have

$$
\begin{aligned}
& \theta \frac{d P_{1}}{d \theta}=\frac{E_{1} E_{2}}{Z} \sin (\gamma+\theta), \\
& \theta \frac{d P_{2}}{d \theta}=-\frac{E_{1} E_{2}}{Z} \sin (\gamma-\theta),
\end{aligned}
$$

and assuming $\cos \theta=\mathrm{I}$, and $\sin \theta$ negligible in comparison with $\cos \theta$, we have

whence

$$
\frac{d^{2} \theta}{d t^{2}}=\frac{E_{1} E_{2}}{\omega Z}\left(\frac{p_{1}^{2}}{K_{1}}+\frac{p_{2}^{2}}{K_{2}}\right) \sin \gamma \times \theta
$$

$$
\theta=2 \pi \sqrt{9.8 \mathrm{I} \frac{\omega}{E I_{8 c} \frac{\sin \gamma}{2}\left(\frac{p_{1}^{2}}{K_{1}}+\frac{p_{2}{ }^{2}}{K_{2}}\right)} .}
$$


In the particular case where the motor is supplied from a circuit of great output-capacity the term $\frac{p_{1}^{2}}{K_{1}}$ can be considered as very small in comparison with $\frac{p_{2}^{2}}{K_{2}}$, and, consequently, the expression for the period of the oscillations, as a function of the constants of the machine and of the inertia of the armature and of the parts mounted on the shaft, is materially increased.

This formula shows that the oscillations are all the more rapid and of lower amplitude the lower the self-induction and the mechanical inertia.

Consequently, from this point of view, it would be more profitable to select motors with a low armature-reactance, a conclusion which we had already reached by another process. The oscillations not only have the effect of causing the motor to fall out of step, and of thus limiting its maximum output, but also of producing oscillations of E.M.F. and current, including change of phase (lag). These effects can manifest themselves in a very objectionable manner, on the circuit and on the generator, by producing fluctuations of voltage which are often important, especially when the reactance of the circuit is large and the circuit is thereby made very sensitive to variations in wattless current.

A synchronous motor which is oscillating can therefore cause trouble to its neighbors on the circuit, and it may even render lighting from that circuit impossible. For this reason, great care should be taken to regulate perfectly the speed and phase of the motor by means of a phase-indicator, before connecting it to the supply-circuit.

Every sudden change in load gives rise to a strong oscillation in the phase-angle, causing $E_{2}$ to go beyond the position of equilibrium corresponding to the new value of the power. If this oscillation comes from a reduction in load, it can cause no inconvenience; if, on the contrary, it is caused by an excess of load, the phase-variation mav, in the first impulse, exceed the angle of stability, and the machine will fall out of synchronism.

Calculation shows (A. Blondel, loc. cit., Lumiere Electrique, Vol. XLV, p. 24) that the relative increase of load $\Delta$ which can be made suddenly, starting from an initial power $\left(-P_{2}\right)_{\theta_{0}}$ does not depend on inertia, but only on the two ratios

$$
\frac{\gamma\left(-P_{2}\right)_{\theta_{0}}}{E_{1}^{2}}=y \text { and } \frac{E_{2}}{E_{1}} \cos \phi=x ;
$$


and the curves in Fig. 60 enable its value in each particular case to be calculated as a function of the data. These curves show that $\Delta$ varies in the inverse ratio of $y$ and in direct ratio of $x$, so long as $z$ is lower than a value near $\frac{1}{2}$. The conditions that insure the best stability in the case of sudden overloads are, therefore, substantially the same as those which insure the maximum theoretical output. - In particular, in the case where $E_{2}=E_{1}$, we should have $\cos \phi=\frac{1}{2}$, whence $m l=r \sqrt{3}$.

The experiments made on a Ganz motor (Experiments made by the Frankfurt technical commission, Upperborn, Lumière Electrique, Vol. XXXVI, p. 3I5), showed that, starting from no-load, a sudden

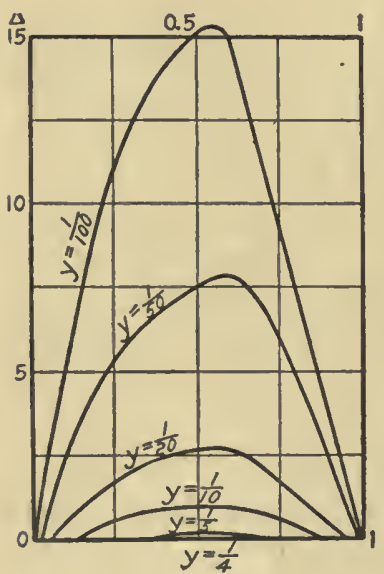

FIG. 60. load equal to 150 per cent of the nominal load may be put on the motor. This load could be much exceeded for alternators of lower inductance, especially if they are provided with dampers on the polepieces.

Damping of Oscillations. The surest way to prevent oscillations of both kinds from attaining an excessive amplitude consists in damping them rapidly. It is an application of the general principle laid down in a masterly manner by Cornu ("On the synchronization of oscillating systems," Journal de Fhysique et Comptes Rendu de l'Académic des Sciences, 3I Mai, I887; Bulletin de la Société des Electriciens), who showed that perfect synchronization can be obtained only when there is damping, i.e., when the speed-variations cause a supplementary expenditure of energy. This supplementary expenditure of energy can only occur to a slight extent in the armature-circuit, but it is possible to bring it about in the field-circuits by allowing eddy currents to be produced in the massive pole-pieces or, better still, in special circuits wound on the fields, and short-circuited. These circuits constitute the damper of Hutin and Leblanc. (C. F. Guilbert, Lumière Electrique, Vol. XLVI, I892, p. 801; and I.eblanc, Bull. de la Soc. Int. des Electriciens, I 898). This damper consists either of bars of copper surrounding the pole-pieces, as shown in Fig. $6 \mathrm{I}$, or, better still, of a series of bars passing through the fields and united on each side by copper rings, as shown in Fig. 62 . 
The explanation of the role of these dampers is made easy by reference to the elementary considerations set forth on page II. When there are no speed-oscillations, the armature-reaction flux undergoes no displacement with respect to the field-poles, and, consequently, it does not generate any induced E.M.F. in the damping circuits. On
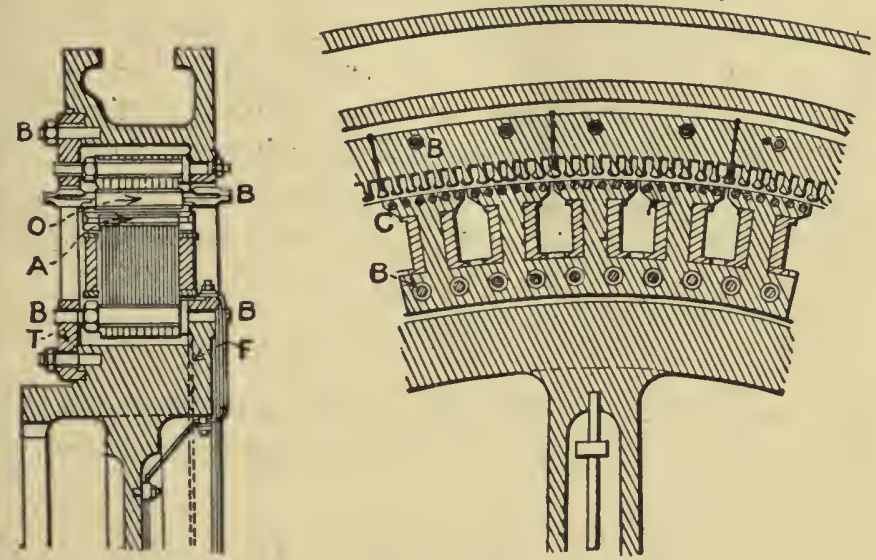

Fic. 6I.

the contrary, any variation of speed producing a flux-oscillation gives rise to eddy currents, which are all the stronger the greater the conductivity of the damping circuits and the greater the extent to which they increase the flux developed by the armature. The energy consumed by the induced currents is what produces the damping. It

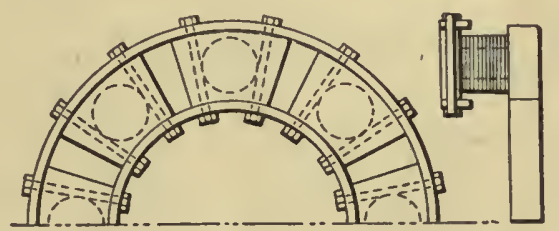

FIG. 62 .

manifests itself by the production of a corrective torque exerted on the armature and which tends to bring the speed to synchronism.

The same explanation applies in the case of a single-phase motor, with this difference, that the inverse revolving flux due to the armature should, in consequence of its high velocity relatively to the damping circuit, produce strong induced currents which almost completely 
neutralize this armature-flux. The result is that the armature-reaction, or the apparent inductance in the armature, is reduced about one-half and, at the same time, the noise of single-phase alternators disappears. The loss of energy is much lower than might be supposed, because it eliminates, incidentally, the hysteresis-effect in the fields. In any case, the damping effect can be regulated easily to an amount that is deemed sufficient, as can be seen in the following manner:

Suppose the motor departs from synchronism; the period of its induced E.M.F. is no longer the value $T$, but a slightly different value, $T^{\prime}$. The coefficient of mutual induction between the armaturecircuit and the damping-circuit, being a periodic function of the angle described by the moving part, can be written in the following form:

$$
M=M_{0} \sin \left(2 \pi \frac{t}{T^{\prime}}-\gamma\right)
$$

in which $r$ is the phase-angle measured from the origin of time.

The current can be represented by the following expression:

$$
I=I_{0} \sin 2 \pi \frac{t}{T}+I_{0}^{\prime} \sin \left(2 \pi \frac{t}{T^{\prime}}-\beta\right) .
$$

The flux sent into the damping-circuit by the induced current, at each instant will be

$$
\begin{aligned}
\Phi=M I=M_{0} I_{0} \sin 2 \pi \frac{t}{T} \sin \left(2 \pi \frac{t}{T^{\prime}}-\gamma\right) \\
\quad+M_{0} I_{0} \sin \left(2 \pi \frac{t}{T^{\prime}}-\beta\right) \sin \left(2 \pi \frac{t}{T^{\prime}}-\gamma\right) .
\end{aligned}
$$

or, substantially, if we confine ourselves to the first term,

$$
=\frac{M_{0} I_{0}}{2}\left\{\begin{array}{l}
-\cos \left[2 \pi\left(\frac{\mathrm{I}}{T}+\frac{\mathrm{I}}{T^{\prime}}\right) t-r\right]+ \\
+\cos \left[2 \pi\left(\frac{\mathrm{I}}{T}-\frac{\mathrm{I}}{T^{\prime}}\right)+r\right]
\end{array}\right\}, \ldots . . .
$$

The corresponding E.M.F. will be

$$
e=-\frac{d \Phi}{d t}=\left\{\begin{array}{r}
\pi\left(\frac{\mathrm{I}}{T}+\frac{\mathrm{I}}{T^{\prime}}\right) M_{0} I_{0} \sin \left[2 \pi\left(\frac{\mathrm{I}}{T}+\frac{\mathrm{I}}{T^{\prime}}\right) t-\gamma\right] \\
-\pi\left(\frac{\mathrm{I}}{T}-\frac{\mathrm{I}}{T^{\prime}}\right) M_{0} I_{0} \sin \left[2 \pi\left(\frac{\mathrm{I}}{T}-\frac{\mathrm{I}}{T^{\prime}}\right) t-\gamma\right]
\end{array}\right\} .
$$


Consequently, if $\rho$ equals the resistance, and $\lambda$.the inductance of the damping circuit, the induced current $i$ is due to the superposition of two currents of two different frequencies:

$$
\begin{aligned}
& i=\left(\frac{\mathrm{I}}{T}+\frac{\mathrm{I}}{T^{\prime}}\right) \frac{M_{0} I_{0} \sin \left[2 \pi\left(\frac{\mathrm{I}}{T}+\frac{\mathrm{I}}{T^{\prime}}\right) i-\psi\right]}{\sqrt{\rho^{2}+\left[2 \pi\left(\frac{\mathrm{I}}{T}+\frac{\mathrm{I}}{T^{\prime}}\right) \lambda\right]^{2}}} \\
& -\pi\left(\frac{\mathrm{I}}{T}-\frac{\mathrm{I}}{T^{\prime}}\right) \frac{M_{0} I_{0} \sin \left[2 \pi\left(\frac{\mathrm{I}}{T}-\frac{\mathrm{I}}{T^{\prime}}\right) l-\psi^{\prime}\right]}{\sqrt{\rho^{2}+\left[2 \pi\left(\frac{\mathrm{I}}{T}-\frac{\mathrm{I}}{T^{\prime}}\right) \lambda\right]^{2}}} .
\end{aligned}
$$

The energy consumed is represented by the integral

$$
W=\int_{0}^{t} e i d t
$$

which contains products of terms having different frequencies. If the integration is supposed to extend over a sufficiently long period, these terms can be neglected in comparison with the others, which alone increase indefinitely. In that case, the value of the mean power expended per second can be written as follows:

$$
\begin{aligned}
P=\left[\pi\left(\frac{\mathrm{I}}{T}+\frac{\mathrm{I}}{T^{\prime}}\right) M_{0} I_{0}\right]^{2} & \frac{\rho}{\rho^{2}+\left[2 \pi\left(\frac{\mathrm{I}}{T}+\frac{\mathrm{I}}{T^{\prime}}\right) \lambda\right]^{2}} \\
& +\left[\pi\left(\frac{\mathrm{I}}{T}-\frac{\mathrm{I}}{T^{\prime}}\right) M_{0} I_{0}\right]^{2} \frac{\rho}{\rho^{2}+\left[2 \pi\left(\frac{\mathrm{I}}{T}-\frac{\mathrm{I}}{T^{\prime}}\right) \lambda\right]^{2}} .
\end{aligned}
$$

The first term is substantially constant, since $T^{\prime}$ remains near $T$. On the contrary, the second term, which vanishes when $T^{\prime}=T$, increases rapidly with the difference of frequencies. It represents the correlative increase of energy consumed, which produces the damping.

Supposing the difference $\frac{\mathrm{I}}{T}-\frac{\mathrm{I}}{T^{\prime}}$ to be very small, the inductance may be neglected, in comparison with the resistance, in the second term. In the first term, on the contrary, the inductance is always very large, in comparison with the resistance, and, consequently, the latter 
may be neglected. The expression for $P$ is therefore practically equal to the following:

$$
P=\rho\left(\frac{M_{0} I_{0}}{2}\right)^{2}+\frac{I}{\rho}\left[\pi\left(\frac{I}{T}-\frac{I}{T^{\prime}}\right) M_{0} I_{0}\right]^{2} .
$$

Consequently, by making $\rho$ very low, as was done by Hutin and Leblanc, the permanent expenditure of energy due to the current of frequency $\frac{I}{T}+\frac{I}{T^{\prime}}$ can be reduced, at the same time that the damping effect due to the current of frequency $\frac{\mathrm{I}}{T}-\frac{\mathrm{I}}{T^{\prime}}$ is amplified.

When the pole-pieces are solid, they already, by themselves, play the role of a damping circuit, and, their resistance $\rho$ being very small, it can be readily understood that the effect thus obtained would be already quite appreciable.

At synchronism, we have $T^{\prime}=T_{1}$, and the preceding expressions (I) and (2) (p. I28) reduce to the form

and

$$
\Phi=\frac{M_{0} I_{0}}{2}\left\{-\cos \left[2 \pi\left(\frac{\mathrm{I}}{T}+\frac{\mathrm{I}}{T^{\prime}}\right) t-\phi\right]+\cos \phi\right\} .
$$

$$
e=\frac{2 \pi}{T} M_{0} I_{0} \sin \left(2 \pi \frac{t}{\frac{T}{2}}-\phi\right) \text {. }
$$

The armature-reaction then produces two fluxes in the field-coils, one being constant, which represents the mean reaction, and the other having a frequency which is double that of the alternators.

Long-Period Oscillations. Long-period oscillations, characterized by periodical variations of current which can be observed on an amperemeter, may occur in consequence of causes which are apart from the peculiarities of the motors themselves. For example, a motor which drives a machine-tool subjected to a variable load, or a motor connected to a generator whose speed varies periodically (for example, a generator driven by a steam engine having a single cylinder), will itself undergo the same corresponding oscillations of currents, which are much slower than the preceding. These slow oscillations have, moreover, the incidental effect of keeping up the real oscillations, and thus they are often the cause of the machines falling out of synchronism.

Other causes more complex, and which are still less well understood, can bring about the systematic and periodic production of this phenom- 
enon of successive falling out of and falling into synchronism. For instance, Hutin and Leblanc (M. Leblanc, Lumière Electrique, Vol. XXXIII, p. 227, and F. Geraldy, ibid., Vol. XLVI, p. 652) showed by an experiment that some Ganz motors of the kind excited by commutated currents did not always attain synchronism, but, in certain cases (especially when running without load), assume an oscillatory movement having long periods. These periods can, in such a case, be attributed to slow alternations of the excitation produced by the current-commutator. This is an additional reason for abandoning definitely this kind of excitation. Certain motors also appear subject to slow oscillations, in consequence of the discontinuity of the $\mathrm{V}$-curve produced by the presence of important upper harmonics in their E.M.F. curve, as already noted above (page I03). 


\section{CHAPTER V}

\section{TESTS OF SYNCHRONOUS MOTORS}

Characteristic Curves. It may be stated, in general, that the characteristic curves of synchronous motors are the same as those of alternators, i.e., the variation of the inducing flux is represented as a func-

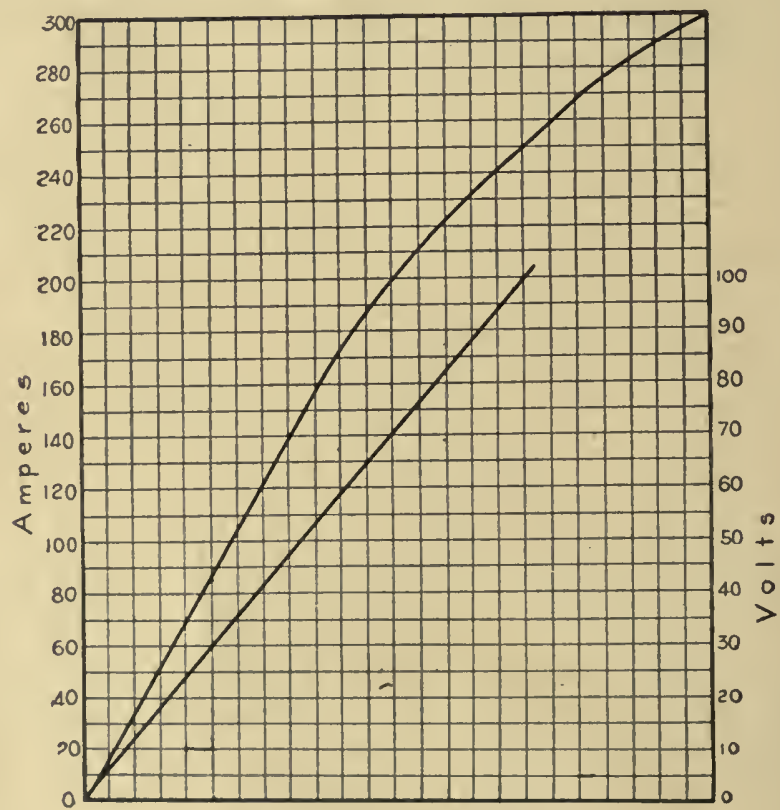

Field-Excitation. Amperes

Fic. 63.-Current consumed by a I5 H.P. synchronous motor, run at constant potential with variable field-excitation. The ordinates represent armature current (amperes) per phase.

tion of the exciting ampere-turns by an "excitation-curve," and the armature-reaction is represented by a short-circuit-curve obtained according to the method of Behn-Eschenburg, by measuring the amperturns necessary to produce increasing currents, when the machine is 
short-circuited on itself and is run at a speed near its normal speed. The short-circuit current will be all the smaller, for a given excitation, the greater the armature-reaction. Since a good stability of operation is only obtained, as already seen, with low armature-reaction, it follows that a good synchronous motor will be characterized by a short-circuit curve which is rapidly ascending such as that shown in Fig. 63, which refers to a synchronous motor with revolving iron of Oerlikon type, described by Kolben (Elektrotechnische Zeitschrift, I9 Dec.

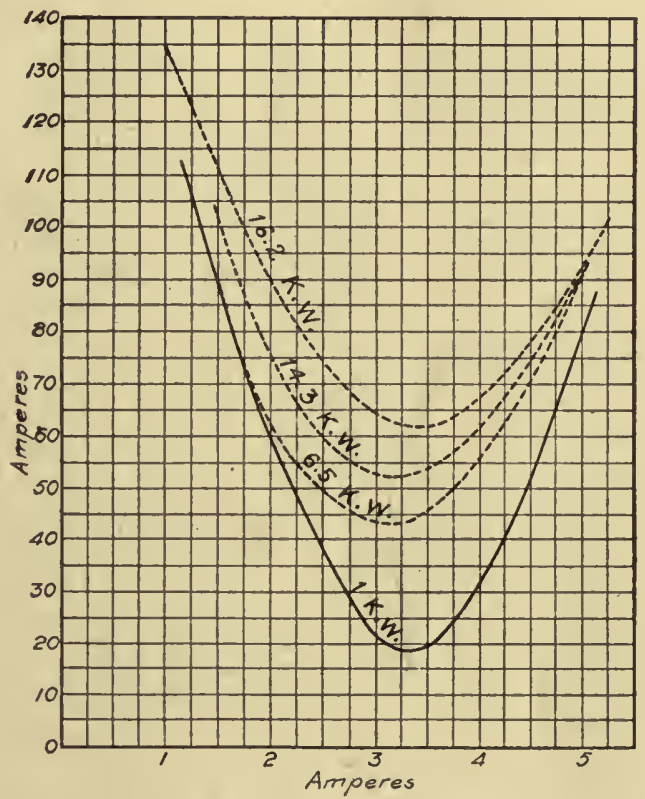

FIG. 64.-Power input for I5 H.P. synchronous motor, run with variable fieldexcitation.

1895). A motor is good, according to this author, when a short-circuit-current equal to the normal operating current is obtained by an excitation giving not more than one-third the normal voltage, for the induced E.M.F. Finally, from what precedes, there exists, for synchronous motors, a third kind of important characteristic curve, the V-curves, of which the theory has already been given. These curves are drawn for variable loads applied by a Prony-brake, by measuring, for each valuc of the excitation, the current-values given by an ampere-meter, and the electric-power values given by a watt-meter. 
Figs. 64 and 65 give, for example, these curves obtained by Kolben for an Oerlikon three-phase synchronous motor of 15 H.P. These curves show that the minimum current

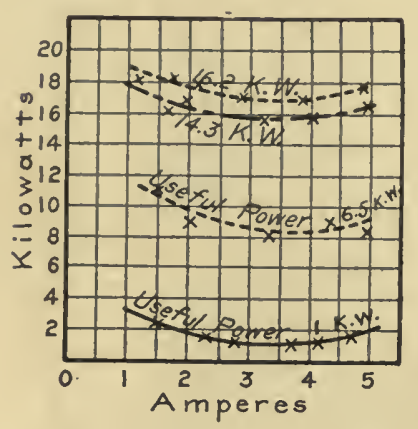

Fic. 65.-Power-curves for threephase 30 H.P. synchronous motor, run with variable fieldexcitation. in the armature is obtained with about the same excitation at all loads, and that the efficiency is almost constant within wide limits. For example, the power consumed at full load, to produce I 4 k.w., scarcely varies between I and 5 amperes of excitation. These favorable conditions characterize a motor having low armature-reaction, Motors having high armature-reaction give rise to eddy currents in the pole-pieces, which involve a considerable increase in the power absorbed, when the current increases in the armature. This peculiarity may be made evident by means of a fourth characteristic curve known as the "characteristic curve of loss by parasite-effect." This curve is obtained by taking as ordinates the power absorbed when running short-circuited, the excitation-currents being taken as abscissæ. Figs. 66 and 67 show, quite clearly, the difference, in this respect, between a motor having a high armaturereaction and a motor having a low armature-reaction. In the latter the eddy currents, which assume great importance, impart a parabolic form to this characteristic curve.

Measurement of Efficiency. The most natural method for the measurement of efficiency is the Prony-brake-dynamometer

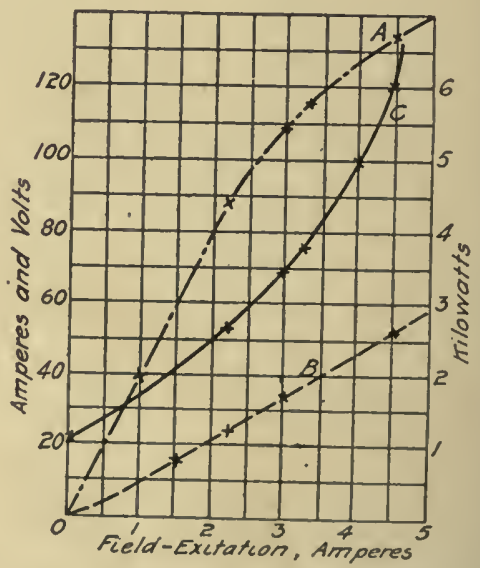

Fic. 66.-Curves for 30 H.P. synchronous motor having high armature-reaction.

$A=$ characteristic curve without load;

$B=$ short-circuit current;

$C=$ power absorbed when short-circuited. method, used in the same way as for a D.C. motor. The resisting torque produced by the brake is measured, and, at the same time, the 
electric power applied at the terminals of the motor is measured by a watt-meter. This method has the objection of requiring the expenditure of too much energy in the case of large motors.

The author's method, described further on, for the study of conditions of operation, then finds its application. In such cases, the generator and motor are mounted and coupled together in such manner that the generator is driven by its pulley, and also tends to be driven by the motor which is connected to the other end of the shaft and which takes the lead and drags the dynamometer. The latter

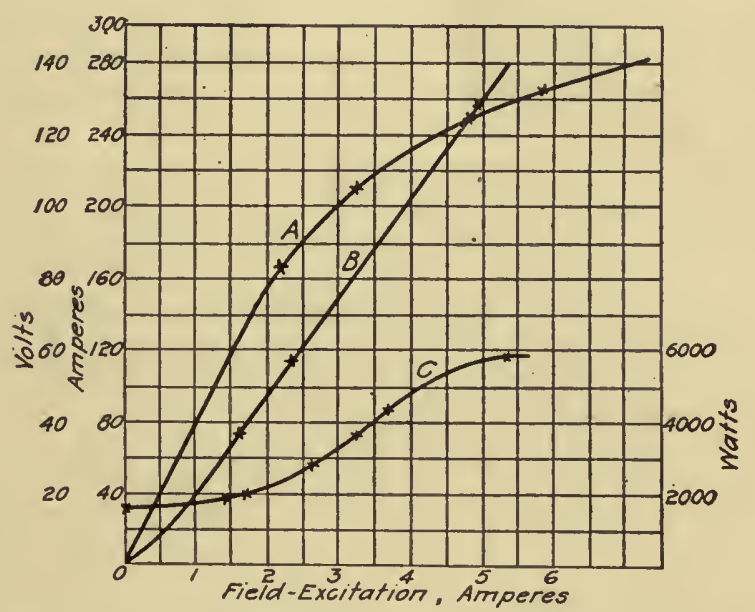

Fic. 67 .-Curves of I $_{5}$ H.P. synchronous motor. Normal voltage 240 volts.

$A=$ characteristic curve without load.

$B=$ short-circuit current.

then measures directly the work done by the motor, and all that is necessary is to measure, by means of a watt-meter, the electric power supplied to the motor by the generator. If we let $C$ equal the resisting torque, $n$ the number of turns per second, and $w$ the electric power measured by the watt-meter, the efficiency, $\eta$, will be expressed by the following equation:

$$
\eta=\frac{2 \pi n C}{w}
$$

The segregation of the different losses, in a synchronous motor, is a much more complex problem than in the case of direct current machines because the parasite effects (hysteresis and eddy currents) in the field-pole pieces depend not only on the induced current and on the 
excitation-current, if the armature-core is slotted, but they also depend on the phase-angle of the induced current, especially in the case of single-phase motors. It can be readily understood that, in the case of single-phase motors, the variation in the reaction-flux in front of the pole is all the greater the more rapidly the armature-current varies in the position of maximum mutual induction. For the same current in the armature, the losses will, therefore, be greater the smaller the phaseangle (lag) of the current.

By the aid of these assumptions, the losses can always be approximately separated, when the conditions of operation are precise, such as when the current-supply has a constant voltage and the excitationcurrent is constant. In such cases, the losses by friction are measured

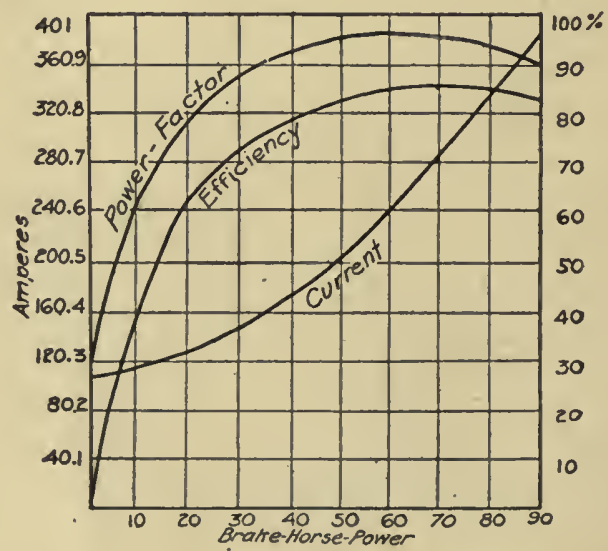

FIg. 68.

by running the machine without excitation, by means of a small D.C. motor whose efficiency curve has been obtained. On exciting the machine, the loss becomes increased by an amount equal to the hysteresis and eddy currents, in the armature and the fields, other than those due to armature-reaction. They may be distinguished, as usual, by running at various speeds. The motor is then run with a load applied at the brake. The difference between the losses noted and those previously measured consists of two portions: the loss due to resistance-heating in the armature, which can be easily calculated from the resistance and the current, which are known; and the increase of loss due to armature-reaction, which is obtained, more or less approximately, by taking the difference. 
Fig. 68 gives an idea of the manner in which the power-factor and the efficiency vary as a function of the load in a synchronous motor.

Experimental Tests. It is interesting to compare the results of experiments with those of calculation. The methods which can be used for that purpose will now be indicated.

It should be understood that a satisfactory agreement between theory and practice can only be hoped for when working with machines having E.M.F.'s of the same wave-form, and as nearly as possible sinusoidal. Owing to the want of this precaution, certain authors (Bedell and Ryan, loc. cit.) have found discontinuities in operation which are inexplicable by the ordinary theory

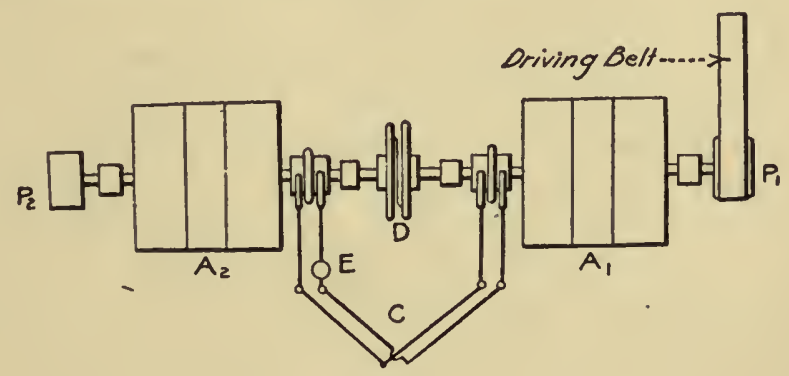

Fig. 69.

The factors to be measured experimentally are the E.M.F.'s induced in the generator and the motor, the current in amperes, and the phaseangles between these various quantities, and also the electrical and mechanical powers applied or developed. The investigation can be more or less complete according to the apparatus available.

The author published, in 1893 ("Theory of the Coupling of Alternators," Bulletin de la Société des Electriciens, 1893, p. 9), a method, based on that of Hopkinson for D.C. dynamos, which has the advantage of requiring only a small amount of mechanical power (Fig. 69).

The two machines, generator $A_{2}$ and motor $A_{1}$, are placed end to end, and the two shafts are joined together by a flange-coupling or a torsion dynamometer $C$ (of type such as used by Mascart, Rateau, Bedell, etc.) so arranged that they can be set in various positions for changing the angle of phase of the two shafts with respect to each other. This phase-angle is known, at rest, and all that is necessary is to add to it the angle of torsion of the dynamometer to have the phase- 
angle when running. One of the alternators is driven by a belt run over its driving pulley, and it drives the other, mechanically through the dynamometer, as well as electrically through the circuit. The alternator which is belt-driven acts as a motor, the other acting as a generator; the torsion-dynamometer connected between the two machines measures the excess of power consumed by the generator over and above the energy recovered. The power applied at the pulley represents the total losses of the two machines operating as a power-transmission system. From these data the efficiencv of transmission may be deduced.

Suppose that, for each phase-angle, the excitation-current and the line-current are read, by means of an electrodynamometer $E$, also that the voltage at the terminals is read, by means of a volt-meter, and that the electric power is read by means of a watt-meter inserted in the circuit, with its shunt-coil connected to the terminals.

By comparing this "actual" power with the "apparent" power, the phase-angle between the internal E.M.F.'s $E_{1}$ and $E_{2}$, is obtained substantially. The magnitudes of these E.M.F.'s are known from the excitation-curves, which are drawn once for all. This method is particularly simple when no transmission-dynamometer is used, when the loads are perfectly steady; but the loads are not then obtained in terms of the mechanical power of the motor.

When it is impossible to couple the two machines rigidly, or when they do not have the same number of poles, it is not so easy to determine the phase-difference or lag between the two motors, and it is then necessary to proceed in one of the two following ways:

(I) It is possible to mount on the shaft of the machines some small alternators serving as "tell-tales." They are arranged in such manner that their E.M.F.'s synchronize in phase with the E.M.F.'s induced when running without load. A triple oscillograph record will then give, directly, for each load, the phase-angles between these F.M.F.'s and the current.

(2) When the machines have not the same number of poles the phase-angles between the induced E.M.F.'s can be measured mechanically by means of the ingenious phase-indicator of Bedell and Moler. The two machines are placed end to end, with their shafts almost touching. The phase-indicator consists of two metallic disks 23 centimeters in diameter and 8 millimeters thick, each fastened to one end of the shaft and provided with curved slits symmetrically disposed in the form of an Archimedean spiral. (Fig. 70.) The disks are 
arranged in such a way that their spirals are pointed in opposite directions. Owing to the form of these curves, the distance from their points of intersection to the center is proportional to the phase-angle between the two disks, or the lag of one with respect to the other. The point of intersection of two slits forms a small opening traversed by rays of light which, in turning, make a circle that expands or contracts in proportion to the phase-angle. The radius of the circle can be measured on a fixed scale which is graduated directly in angles.

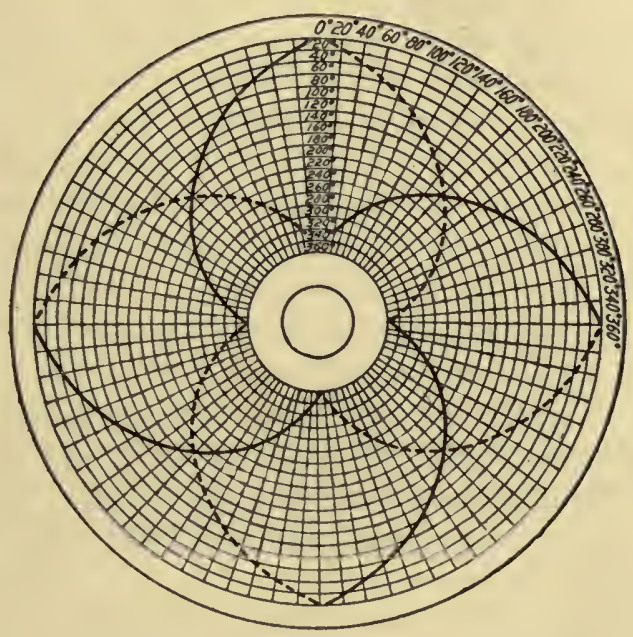

FIG. 70.

Bedell and Ryan have used this method to determine the phaseangle between E.M.F.'s, by bringing this phase-angle to zero when the poles are in line. The currents are measured by means of an electrodynamometer. The E.M.F.'s are deduced from the excitationcurves. Their vectors are then drawn with an angle between them equal to the measured phase-angle, and, from these, the resultant vector is obtained, in magnitude and in phase, by completing the parallelogram. The current is known, in magnitude. Its phase, with respect to the resultant E.M.F., is deduced from the reactance, which is known by means of the expression

$$
\tan \gamma=\frac{\text { reactance }}{\text { resistance }}
$$


or it can be deduced from an oscillograph diagram. We then have all that is necessary to draw the diagram. The results are sufficiently concordant with the theory, but they indicate no new phenomenon, except the discontinuity already mentioned. For more complete details see the author's original paper.

Advantages and Disadvantages of Synchronous Motors. The characteristic features of synchronous motors, constituting their advantages and disadvantages, may be summarized as follows:

(I) Their construction is as simple as that of alternators, and it enables them to be connected directly to high voltage circuits.

(2) Their specific output and their efficiency are also the same, and are quite satisfactory.

(3) When once put in operation they have perfectly uniform speed.

(4) Their power-factor is as good as may be desired when their E.M.F. is properly selected. By using them, all reactive currents can be eliminated, and currents can even be made to lead in phase, by suitable regulation of the excitation.

(5) It is difficult to start these motors, especially single-phase motors; special methods and apparatus must be employed and also an idler-pulley or clutch. Even when starting without load, which is the only practical way, a very high current is required.

(6) Speed-regulation is impossible.

(7) A very sudden overload causes the motor to fall out of phase and brings about a short-circuit.

(8) Any irregularity of operation of the generator causes great oscillations of speed ("pumping") which make the voltage of the system fluctuate. If the excitation is not well regulated, the motor produces a more or less important reactive current, which may bring about a drop of voltage in the system.

(9) If the E.M.F. curve of the motor is different from that of the supply-system, harmonics will be produced, which will uselessly increase the line-current and compromise the stability of operation. Stability of operation can only be attained by the addition of inductance to the circuit; and it remains weak, at best.

(Io) A synchronous motor reacts more or less on the rest of the system, to which it can communicate its oscillations, or of which it can distort the E.M.F. curve. The construction of synchronous motors should therefore be carefully studied, not only with reference to their operation by themselves, alone, but with reference to their effect on the distributing system from which they are to be supplied. 


\section{CHAPTER VI}

\section{OTHER MOTORS OPERATING SYNCHRONOUSLY WITHOUT DIRECT CURRENT EXCITATION}

It is possible to devise other motors which, once brought to a suitable speed, can continue to operate synchronously, without excitation by a direct current or by a commutated pulsating current. These motors, not being used industrially, constitute, properly speaking, theoretical curiosities, but it is interesting, in order to give a general idea of the whole subject, to indicate their principles.

Mention can be made of two types of such motors; synchronous motors without any excitation, known as "reaction-motors," and synchronous motors with alternating-current excitation, or alternatingfield motors.

Reaction Synchronous Motors. - It is natural to suppose that the constant armature-reaction-field of polyphase motors can be used as an inducing field. It is sufficient, for this purpose, that the armature should have a large number of windings to produce a large number of ampere-turns, and that the current passing through it should be much out of phase, in order that the magnetizing turns may coincide with those of the field-poles. Moreover, the air-gaps should, obviously, be very small, to reduce the reluctance of the inducing circuit. The self-excitation of the latter is then only an exaggeration of the effect of the lagging reactive current, which has already been mentioned several times. The operation of such a motor is therefore quite possible, even at light loads; but the inductance of the armature has been increased at the same time as its reaction on the field; and the consequence is that the stability and, moreover, the power-factor of the current used, will be small, owing to the great lag of this current. Such a motor would, therefore, be bad from a commercial point of view.

The conditions of operation can be easily ascertained by making use of the diagram on page 94, in which the E.M.F. due to external excitation is supposed to be zero.

The operation of motors on the reaction-principle is not limited 
to polyphase motors, but it can also be accomplished, although with more difficulty, in the case of single-phase motors. The phenomenon was first observed as early as 1874 , by Siemens of London, who found that he could suppress the excitation of a single-phase motor without stopping it. The explanation is simple if we refer to the use already made of the theorem of Leblanc, i.e., that the alternating field of the armature-reaction can be replaced by two fields, each of half the strength, rotating in opposite directions, one of which remains in front of the field-poles and magnetizes them, the other being a useless parasite current, rotating with twice the speed of the poles and producing no effect (except eddy currents).

Another explanation can also be given, based on the variation of the coefficient of self-induction of the armature at each period during its rotation. In consequence of this variation the reaction-flux $l i$ is a maximum when the armature coils are exactly in front of the poles.

This position is therefore that which the armature would tend to take if a direct current were passing through it. Hence, if the machine is driven by hand, it will be sufficient that the motion may continue, as in certain toys, to break or to weaken the current each time that the coils move away from a pole, or that it may close the circuit, or strengthen the current, each time that they approach the next pole. Now this effect is precisely produced with an alternating current (because the direction of the current is of no importance here) as soon as the maximum of the periodic current is made to lead in phase with respect to the maximum of $l$, as can be easily seen. The sum of the effects which attract the armature in the direction of motion during a period is then greater than the sum of the contrary effects.

The motion therefore continues, and it is easy to see that it may be steady, because every acceleration of the motion tends to diminish the phase-angle between $i$ and $l$, and, consequently, tends to diminish the dissymmetrical action, while retardation increases the phase-angle, and, consequently, increases the useful action of the current.

These considerations can be easily verified by calculation by referring to the equation

$$
u=e-r i-\omega l \frac{d i}{d t}
$$

in which we will let $e=0$, to take into account the fact that there is no excitation. The alternator having a variable self-induction

$$
l=l^{\prime}+\lambda \cos 2 \omega t,
$$


and supposing the current passing through it from the inside to the outside to be sinusoidal, and to be out of phase by the angle $\psi$ with respect to the position of coincidence between the poles, we will have

$$
I=I_{0} \sin (\omega t-\gamma)
$$

We will therefore have, for the voltage at the terminals,

$$
\begin{aligned}
u= & -r I_{0} \sin (\omega t-\psi) \\
& -\omega\left[\left(l^{\prime}-\frac{\lambda}{2}\right) I_{0} \cos \psi \cos \omega t+\left(l^{\prime}+\frac{\lambda}{2}\right) I_{0} \sin \psi \sin \omega t\right],
\end{aligned}
$$

the harmonics of the order $3 \omega t$ introduced by the pulsation of $l$ being neglected.

This equation is represented by the diagram in Fig. 7I. It is seen

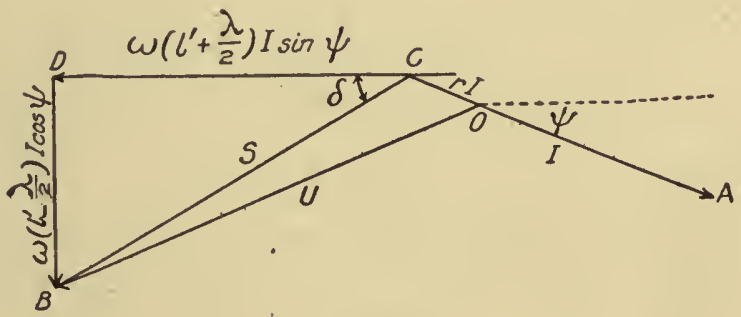

FIG. 7 I.

that the voltage $U$ at the terminals is the geometrical resultant of the vectors

$$
\left[-r I,-\omega\left(l^{\prime}+\frac{\lambda}{2}\right) I,-\omega\left(l^{\prime}-\frac{\lambda}{2}\right) I \text { (perpendicular) }\right] .
$$

In this diagram the lag $\psi$ of the current is defined as the interval between the zero-value of the current and the position of coincidence of the poles.

It is seen that the E.M.F. of self-inductance $S$ makes an obtuse angle with the current $I$ so long as $\psi$ is comprised between $\circ$ and $\frac{\pi}{2}$ (the two vectors being in opposition when $\psi=0$ ), and that it is in quadrature when $\phi=\frac{\pi}{2}$. 
The expression for the mean electric power can be easily obtained, according to the usual rule, by multiplying the vector $S$ by the projection of $I$ on $S$ ( $I$ and $S$ being effective values). We then have

$$
\begin{aligned}
& P=S I \cos A C B \\
& =I^{2} \omega \cos (\pi-\psi-\delta) \sqrt{\left(l^{\prime}+\frac{\lambda}{2}\right)^{2} \sin ^{2} \psi+\left(l^{\prime}-\frac{\lambda}{2}\right)^{2} \cos ^{2} \psi},
\end{aligned}
$$

on the assumption that

$$
\tan \delta=\frac{\left(l^{\prime}-\frac{\lambda}{2}\right) \cos \psi}{\left(l^{\prime}+\frac{\lambda}{2}\right) \sin \psi}
$$

The value of the power-factor, obtained from the preceding, is

or

$$
\begin{aligned}
\cos (\pi-\psi-\delta) & =-\cos \phi \cos \delta+\sin \psi \sin \delta \\
& =\frac{-\lambda \cos \psi \sin \psi}{\sqrt{\left(l^{\prime}+\frac{\lambda}{2}\right)^{2} \sin ^{2} \psi+\left(l^{\prime}-\frac{\lambda}{2}\right)^{2} \cos ^{2} \psi}} \\
& =\frac{-\lambda \sin 2 \psi}{\sqrt{l^{\prime 2}+\frac{\lambda^{2}}{4}-\lambda l^{\prime} \cos 2 \psi}}
\end{aligned}
$$

whence

$$
P=-\omega \lambda I^{2} \sin \psi \cos \psi=-\frac{\omega \lambda I^{2} \sin 2 \psi}{2} \text {. }
$$

The power is negative and therefore the alternator operates as a motor (consuming electrical energy and producing mechanical power) when the value of $\psi$ is comprised between $\circ$ and $\frac{\pi}{2}$; and it operates as generator (producing electrical energy and consuming mechanical power) for values of $\psi$ comprised between $\circ$ and $-\frac{\pi}{2}$, i.e., when the current is leading.

The power is a maximum for

$$
\psi= \pm \frac{\pi}{4}
$$

and its absolute value is

$$
P_{\max }=\frac{\omega \lambda I^{2}}{2}=\frac{\omega \lambda I}{2} \times I .
$$


This power is necessarily very low, since $\lambda$ is never more than a fraction of the mean self-induction $l^{\prime}$, and since the E.M.F. which is utilized, $\frac{\omega \lambda l}{2}$, is itself only a small fraction of the E.M.F. of self-induction of the alternator, which latter is always lower than the E.M.F. that would be generated if the alternator were excited by its fields. It can be easily seen that the power-factor always remains very low. Moreover, this motor has the same disadvantage as polyphase reactionmotors. For this reason, this method of using alternators does not appear susceptible of practical application.

Synchronous Motor with Alternating Fields. The invention and the theory of a synchronous motor with alternating fields are due to Galileo Ferraris (see Memoir prescnted to the Academy of Sciences of Turin, Dec. 1893, and also Industrie Electrique, 10 Juin, 1894). In this apparatus the fields and armature are excited in series or in shunt by alternating currents taken from the same source, and the armature is previously brought to a speed double that which would be suitable for a synchronous motor with constant fields. The motor then runs synchronously, and it can be lightly loaded.

The operation of such a motor is easily explained by the aid of the theorem of Leblanc. Let $2 p$ equal the number of poles and $\omega$ the speed of pulsation of the currents. The alternating field (the field winding being supposed stationary) can be expressed fictitiously by two fields, $H_{1}, H_{2}$, one rotating to the right, the other to the left, with the contrary speeds $+\frac{\omega}{p}$ and $-\frac{\omega}{p}$. A speed equal to $\frac{2 \omega}{p}$ is given to the armature in one direction; the alternating field which the armature produces is itself also equivalent to two revolving fields having the velocities $+\frac{\omega}{p}$ and $-\frac{\omega}{p}$ relatively to the armature. The corresponding absolute speeds will be

First field, $M_{1}, \quad \frac{2 \omega}{p}+\frac{\omega}{p}=\frac{3 \omega}{p}$ to the right.

Second field, $M_{2}, \frac{2 \omega}{p}-\frac{\omega}{p}=\frac{\omega}{p}$ to the right.

The field $M_{1}$ will have no action on the fields $H_{1}$ and $H_{2}$, whose speeds are either different or contrary. Likewise, $M_{2}$ will have no 
action on $H_{1}$. On the contrary, $H_{2}$ and $M_{2}$, both rotating to the right with the same speed $\frac{\omega}{p}$, will produce a regular torque.

A motor of this type can be started more or less well by the methods of starting already described for ordinary synchronous motors. If the motor is started by means of an induction-motor for two-phase currents, it is well to group the stator-coils so as to reduce the number of poles by one-half, and thus obtain the double speed which is necessary.

A disadvantage of this system is that it gives rise to upper harmonics in the induced E.M.F. of the motor. Moreover, the field $M_{1}$ of the armature will induce a harmonic of frequency $\left.3^{\prime \prime}\right) t$ in the fieldcircuit, while the fields will give, in the armature, an E.M.F. of the form

$$
A \sin \omega t \sin 2 \omega t=\frac{A}{2}(\cos \omega t-\cos 3 \omega t)
$$

i.e., also a harmonic of the same frequency, $3 \omega t$.

Besides, in consequence of the high self-induction, the powerfactor is necessarily very low.

Another type of single-phase synchronous motor with alternating field can also be obtained by employing, in the armature. currents commutated by means of a shell-commutator analogous to that used for the Ganz motors previously described. When once synchronism has been attained, the effect of this commutator is to invert the current in the armature so that the torque resulting from the action of the field on the armature always retains the same sign. This motor is about equivalent to the motor with pulsating field, since the two elements are simply inverted and the armature can be considered as a revolving field. The only difference results from the relative importance of the ampere-turns of the armature in the fields. 


\title{
CHAPTER VII
}

\section{BIPOLAR DIAGRAM OF THE SECOND KIND IN AMPERE-TURNS}

\author{
By Prof. C. A. Adams, S.B., E.E.
}

\section{INTRODUCTION}

In order to make plain the transition from Fig. 27, which is fundamentally an E.M.F. diagram, to the corresponding M.M.F. Diagram, it will be necessary to develop what may be called the General Vector Diagram of the Synchronous Motor.

Referring to Fig. $72, R$ represents in magnitude and space phase ${ }^{1}$ or direction, the resultant of the field M.M.F. $F$ and the armature M.M.F. A. The space phase of $F$ is its direction reduced to a twopole equivalent; i.e., the direction of the axis of the field poles in two-pole diagram. The space phase of $A$ is, for a single-phase machine, the average direction (with respect to field structure) of the armature M.M.F., and coincides with the direction of this M.M.F. when the current is a maximum, right-handed or clockwise rotation of the armature being assumed. In a polyphase machine the resultant of the M.M.F.'s of the several armature phases is approximately constant in magnitude and direction for all parts of a revolution. The time phase of the armature current $I$ may also be represented by the direction of $A$; and by choosing a proper scale of amperes, the vectors $I$ and $A$ may be made identical.

Assuming that the reluctance of the magnetic circuit through the armature and air-gap is the same in all radial directions, there will result a flux, $\Phi$, in the same direction as $R$. The rotation of the armature through this flux will cause to be induced an E.M.F. $E_{2}^{\prime}$ in magnitude proportional to, and in time phase $90^{\circ}$ behind, $\Phi$. The

${ }^{1}$ A similar analysis for the alternator is given in greater detail in the Harvard Engineering Journal for January, I903. 
vector $E_{2}{ }^{\prime}$ also represents (on the space phase diagram with righthanded ${ }^{1}$ armature rotation) the direction of the axis of an armature coil when the induced E.M.F. is a maximum, just as the vector $I$ represents both the time phase of the armature current and the direction of the axis of an armature coil when its current is a maximum, which is thus the direction or space phase of the armature M.M.F. A. The vector $E_{1}{ }^{\prime}$ drawn equal to $E_{2}{ }^{\prime}$ and in opposite phase; represents that part of the impressed E.M.F. $E_{1}$ necessary to balance the induced E.M.F. $\left(E_{2}{ }^{\prime}\right)$. The impressed E.M.F. $\left(E_{1}\right)$ must then be equal to $E_{1}{ }^{\prime}$ plus the E.M.F. Ir, in phase with $I$, consumed by the armature

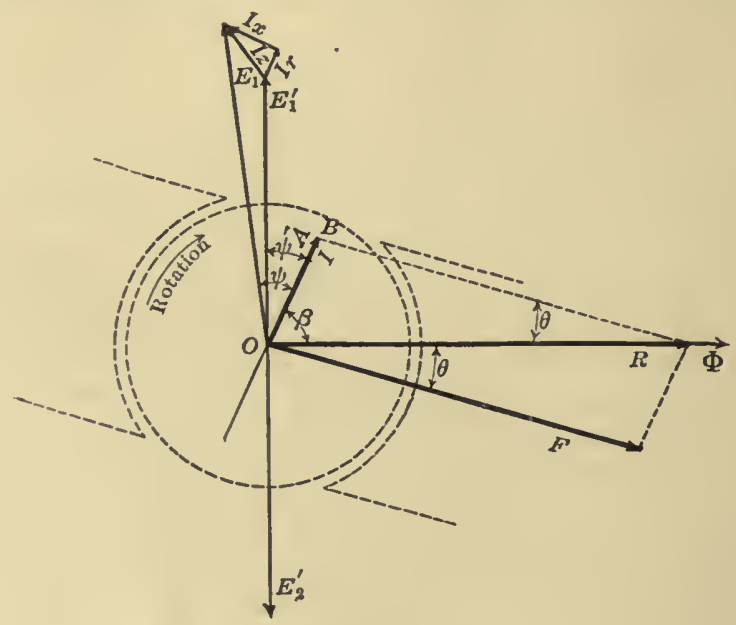

FIG. 72.

resistance, and the E.M.F. $I x, 90^{\circ}$ ahead of $I$, consumed by the leakage reactance of the armature.

The diagram of Fig. 72 gives a fairly complete representation of the relations involved in the operation of the synchronous motor. Two assumptions are involved, however, which should be kept in mind in case of a quantitative analysis: first, it is assumed that the flux $\Phi$ is in the same direction as the resultant M.M.F. $R$; but in the

${ }^{1}$ It will be observed that the right-handed or clockwise armature rotation here assumed for the space phase interpretation of the diagram corresponds to the customary left-handed vector rotation in the time phase diagram. In the case of a revolving field type of machine, the field must revolve left-handedly, in order to give the same relative motion between armature and field. 
salient pole ${ }^{1}$ machine the reluctance of the magnetic circuit through the armature is least in the direction of $F$ and very much larger at right angles thereto; the flux will therefore tend to lie inside of $R$, i.e., on the side towards $F$ : second, $F$ is taken as the total M.M.F. of the field coils per complete magnetic circuit; but a part of $F$ is consumed by the reluctance of the field cores and yoke, and is thus not available to compound with $A$ in the armature space. The errors due to these two assumptions partly neutralize each other as far as the direction of $\Phi$ is concerned, since the second throws it too far out and the first tends to bring it back.

But even this diagram with its approximations is too complex to be of service in getting a clear bird's-eye view of synchronous motor operation, although it is an excellent starting point for further approximations, and will prove very useful for reference purposes.

The two familiar approximations are obtained as follows:

\section{Diagram Transformations}

In any dynamo-electric machine there are in general two M.M.F.'s, those of the field and armature. In computing the E.M.F. of such a machine, it is possible either to consider the resultant of these two M.M.F.'s and the corresponding flux, or to consider the fluxes which would be produced by the two M.M.F.'s if acting separately, and to find the resultant of these two fluxes. The results of the two methods of procedure are identical only when the reluctances of the magnetic circuits in which the several M.M.F.'s act are equal. This condition, though never present except in a very roughly approximate degree, is nevertheless a very convenient one to assume for purposes of approximate analysis. With this assumption, the various fluxes in any case will be proportional to their several M.M.F.'s and in the same direction or space phase; moreover, at constant speed of rotation the E.M.F.'s generated in the armature by cutting through these fluxes will be proportional thereto and in space and time quadrature therewith.

It will be legitimate, therefore, under the above-mentioned assumption, to substitute for any M.M.F. its corresponding flux or E.M.F., or to substitute for any E.M.F. its corresponding flux or

${ }^{1}$ The most accurate diagram for the salient pole machine is Professor Blondel's Two-reaction Diagram employed in Chapter III; but this does not lend itself readily to a simple visualization of the problem in hand, or to simple calculations. 
M.M.F., since these are proportional to each other in magnitude and bear to each other a fixed phase relation.

E.g., in Fig. 72 there are shown vectors which represent three M.M.F.'s, $A, . F$, and their resultant $R$, each one of which would, under the above assumption, produce a flux of the same phase and proportional in magnitude. These fluxes $\Phi_{A} \Phi_{F}$ and their resultant $\Phi$ are shown in Fig. 73, as well as the corresponding E.M.F.'s $E_{A}{ }^{\prime}$, $E_{F}{ }^{\prime}$, and $E_{2}{ }^{\prime}$, which would be induced by the cutting of the armature conductors through these fluxes, and which are $90^{\circ}$ behind their respective fluxes. There are thus three M.M.F.'s, three fluxes, and three E.M.F.'s, each set being proportional to the other two, and

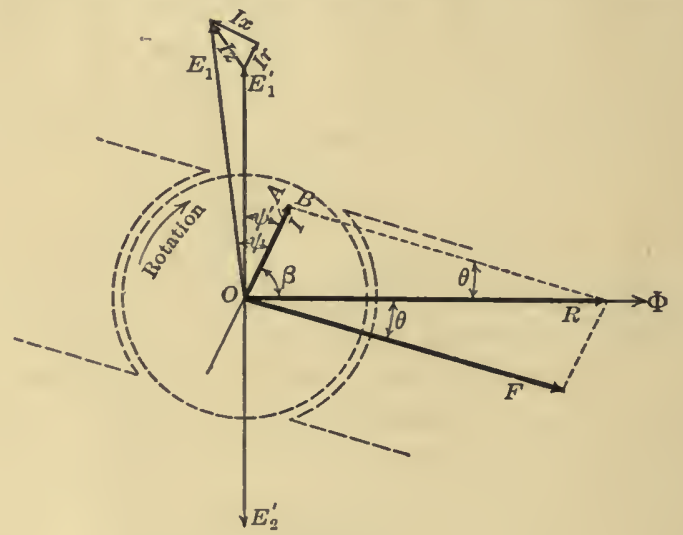

Fic. 72.

the three triangles representing the relations between the elements of the three sets being similar.

\section{E.M.F. Diagram}

In Fig. $73 E_{F}, E_{A}$, and $E_{1}{ }^{\prime}$ are the parts of the impressed E.M.F. consumed by the induced E.M.F.'s $E_{F}{ }^{\prime}, E_{A}{ }^{\prime}$, and $E_{2}^{\prime}$ respectively, just as $I r$ and $I x$ are the parts of the induced E.M.F. consumed by resistance and leakage reactance. Moreover, since $E_{A}$ is proportional to and in quadrature with $A$ (and therefore $I$ ), it is in phase with $I x$ and may be written,

$$
E_{A}=I x_{A},
$$

where $x_{A}$ (the constant of proportionality) is an equivalent reactance representing the armature M.M.F. The sum $x+x_{A}=X$ is called 
the synchronous reactance, and the corresponding impedance $Z=\sqrt{r^{2}+X^{2}}$ is called the synchronous impedance. The use of these quantities reduces the synchronous motor or alternator to a very simple basis, although at the expense of accuracy.

It is then possible to subdivide the impressed E.M.F. $E_{1}$ into three components; $E_{F}$, necessary to balance the counter E.M.F. $E_{F}^{\prime}$ which is due to the excitation $F$ considered as acting alone; Ir consumed by resistance; and $I X$ consumed by the synchronous reactance $X$ : or, the synchronous impedance E.M.F. $I Z$ may be looked

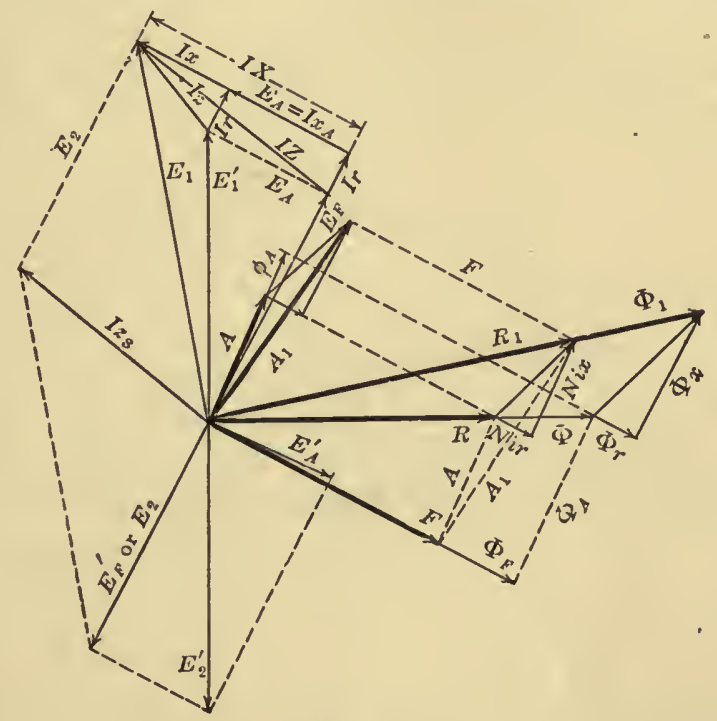

FIG. 73 .

upon as the resultant of the impressed E.M.F. $E_{1}$ and the counter E.M.F. $E_{F}{ }^{\prime}$. This is shown more clearly in the simplified E.M.F diagram of Fig. 74, where $E_{2}$ corresponds to the $E_{F}{ }^{\prime}$ of Fig. 73, and $E=I Z$. The current $I$ will then lag behind the resultant E.M.F. $E$ by an angle whose tangent is $\frac{X}{r}$.

The transformation from Fig. 72 to Fig. 73 consists in substituting for the armature M.M.F. $A$, an E.M.F. $E_{A}{ }^{\prime}$, which would be induced in the armature by cutting the flux $\Phi_{A}$, which in turn would be produced by the M.M.F. $A$ if acting in a magnetic circuit of the same reluctance as that in which $R$ produces $\Phi$. 
The triangle $E_{1} E_{2} E$ of Fig. 74 is the E.M.F. equivalent of the M.M.F. triangle $R_{1} F A_{1}$ of Fig. 73; or, if $r$ and $x$ be neglected, it would reduce to the E.M.F. equivalent of the triangle $R F A$ of Fig. 73 .

This is the basis of the diagrams of Chapter II, which are essentially E.M.F. diagrams.

The M.M.F. or ampere-turn diagram is similarly obtained from the general diagram by substituting for the E.M.F.'s $I r$ and $I x$ their equivalent M.M.F.'s $N i_{r}$ and $N i_{x}$; i.e., $N i_{r}$ and $N i_{x}$ are the M.M.F.'s which would produce the fluxes $\Phi_{r}$ and $\Phi_{x}$, the cutting of which by the armature conductors would induce E.M.F.'s equal and opposite to $I r$ and $I x$ (see Fig. 73), where $R_{1}$ and $\Phi_{1}$ are the M.M.F. and flux corresponding to the impressed E.M.F. $E_{1}$, and $A_{1}$ the M.M.F.

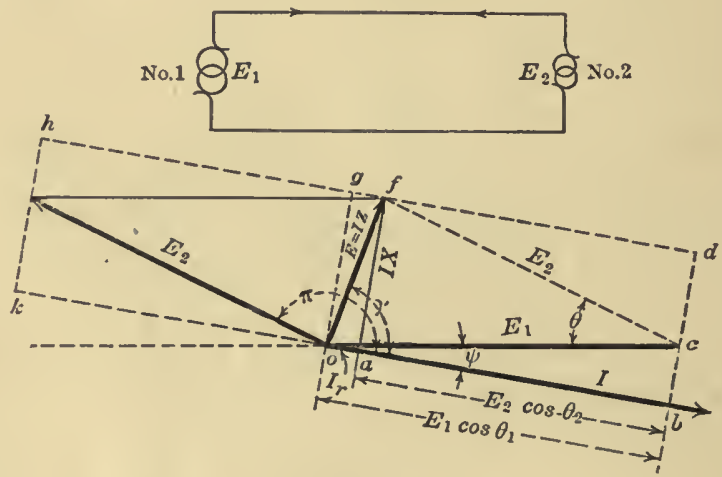

Fig. 74 .

corresponding to the E.M.F. IZ. Thus the M.M.F. rectangle $A_{1} R_{1} F$ is similar and equivalent to the E.M.F. rectangle $I Z E_{1} E_{F}$, and the M.M.F. triangle $A_{1} F R_{1}$ of Fig. 75 is similar and equivalent to the E.M.F. triangle $E E_{2} E_{1}$ of Fig. 74 .

It is thus evident that with constant impressed E.M.F., constant load and variable excitation, the point $B$, Figs. 75 and 76 , will lie on the arc of the circle as does $A_{2}$ in Fig. 27 ; but it will be more interesting to go back to fundamental principles and to make this proof independent of that for Fig. 27.

In any direct-current dynamo-electric machine the electromagnetic torque is proportional to the product of the ampere-turns on the armature and the flux upon which the armature current reacts. In an alternating current machine the space phase of the armature 
current also enters. E.g., in Fig. 75 the armature current in every coil reaches its maximum value when the axis of the coil has the direction of the vector $A$ or $I$; i.e., when the plane of the coil is in the line $\overline{O b}$, which differs from the direction of the flux $\Phi$ by the angle $\psi^{\prime}$. Thus the torque-producing effect of the current is reduced from its maximum possible value, in the ratio $\cos \psi^{\prime}$, and the torque is

$$
T=k_{T} A \Phi \cos \psi^{\prime}
$$

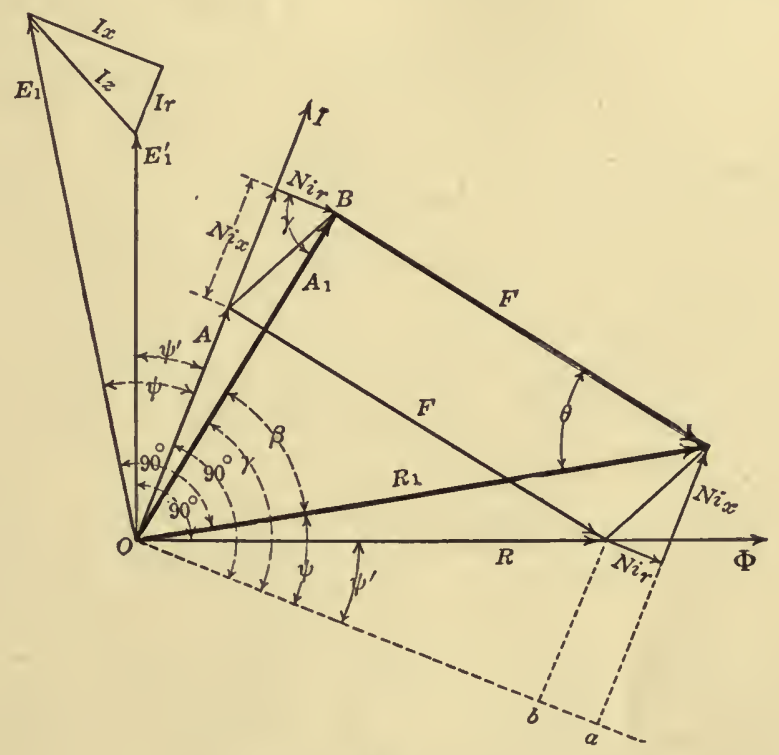

Fig. 75 .

where $k_{T}$ is the constant of proportionality. But

$$
\Phi=\frac{4 \pi}{10} \frac{R}{R}
$$

where $R$ is the reluctance of the magnetic circuit and may for the present be assumed constant.

Then the output (which is proportional to the torque at constant speed) may be written

$$
P_{2}=k_{2} A R \cos \psi^{\prime}, \cdot \text {. . . . . . }
$$


where $k_{2}$ is a constant. But,

$$
R \cos \psi^{\prime}=R_{1} \cos \psi-A_{1} \cos \gamma,
$$

where $\psi$ is the phase difference between $E_{1}$ and $I$, and

$$
\cos \gamma=N i_{r} \div A_{1}=r \div Z
$$

also $A_{1}$ is proportional to $A$ (on the assumption of constant reluctance in all directions through the armature, see "Diagram Transformations," page 149 , etc., therefore

$$
P_{2}=K_{2}\left(A_{1} R_{1} \cos \psi-A_{1}^{2} \cos \gamma\right), \quad . \quad . \quad .
$$

where $K_{2}$ is the new constant of proportionality.

Referring now to Fig. 76, the isosceles triangle $O A C$ is constructed on $R_{1}$ as base, with $\gamma\left(=\tan ^{-1} \frac{A+N i_{x}}{N i_{\tau}}=\tan ^{-1} \frac{X}{r}\right)$ as the base angle. The notation is the same as in the preceding figures.

Locate the point $B$ by the rectangular coordinates $u$ and $v$ measured along the axes $\overline{O C}$ and $\overline{O D}$ (perpendicular to $\overrightarrow{O C}$ ). Then $u=A_{1} \cos \psi$, $A_{1}^{2}=u^{2}+v^{2}$ and

$$
\frac{P_{2}}{K_{2}}=R_{1} u-\left(u^{2}+v^{2}\right) \cos \gamma
$$

or

$$
v^{2}+\left(u-\frac{R_{1}}{2 \cos \gamma}\right)^{2}=\left(\frac{I}{\cos \gamma}\right)^{2}\left[\left(\frac{R_{1}}{2}\right)^{2}-\frac{P_{2} \cos \gamma}{K_{2}}\right] .
$$

This equation has exactly the same form as equation (See Ch. II, near Eq. (Ig)) and its interpretation is likewise the same. The radius of the constant-power circles is

$$
\text { Radius }=\frac{I}{\cos \gamma} \sqrt{\left(\frac{R_{1}}{2}\right)^{2}-\frac{P_{2} \cos \gamma}{K_{2}}} .
$$

The general interpretation of Fig. 76 is identical to that of Fig. 27, but the method of quantitative application is not quite so obvious. The simplest method is as follows:

Since $A_{1}, F$, and $R_{1}$ are measured in ampere-turns, they may in any given case be expressed in terms of the equivalent field amperes, $R_{1}$ being the field current taken from the saturation curve at the point corresponding to the impressed E.M.F. $E_{1}, F$ being the actual operating field current, and $A_{1}$ the field current-taken from the 
short-circuit curve at the point corresponding to the actual armature current $I$. The reason for this last step is obvious when it is recalled that when the machine in question is operated as generator on short circuit, $E_{1}$ and $R_{1}$ are zero and $A_{1}=F$. This is in fact the definition of $A_{1}$.

Equation (5) gives the radii of the constant power circles in ampere-turns; but it is somewhat simpler to compute these radii from the formula given for the E.M.F. diagram, Eq. (I9), Chapter II. This formula may be written

$$
\text { Radius (volts) }=Z \sqrt{\left(\frac{E_{1}}{2 r}\right)^{2}-\frac{P_{2}}{r}}, \ldots . . . \quad .
$$

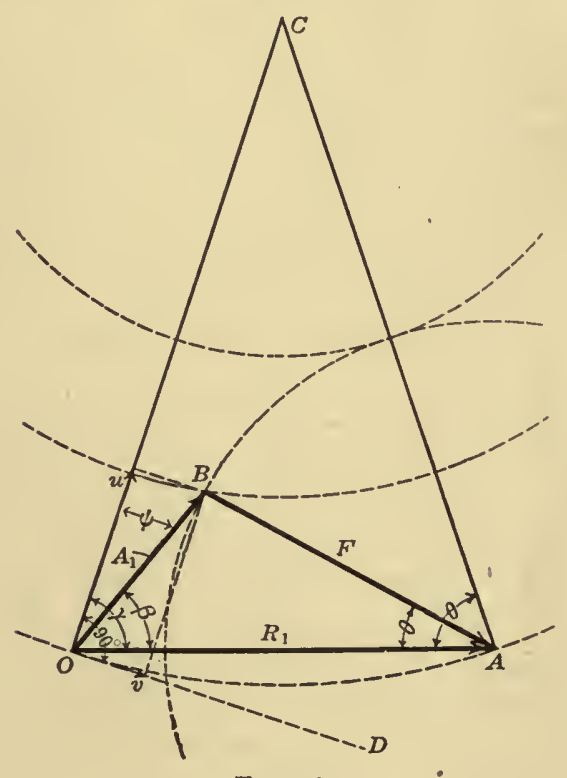

FIG. 76.

or in armature amperes,

$$
\text { Radius (armature amperes) }=\sqrt{\left(\frac{E_{1}}{2 r}\right)^{2}-\frac{P_{2}}{r}} . .
$$

Designate by $k_{a f}$ the ratio of short-circuit armature amperes to the corresponding field amperes as taken from the short-circuit curve. Then the radius in field amperes is

$$
\text { Radius (field amperes) }=\frac{\mathrm{I}}{k_{a j}} \sqrt{\left(\frac{E_{1}}{2 r}\right)^{2}-\frac{P_{2}}{r}} . .
$$


When $P_{2}=O$ the circle passes through $O$ and $A$, and the corresponding radius is,

$$
\text { Radius (field amperes, } P_{2}=O \text { ) }=\frac{E_{1}}{2 k_{a j} r}=\overline{C O} \text {. }
$$

This together with $R_{1}$ determines the isosceles triangle of Fig. 76, although the base angles $\theta$ are the same as for the E.M.F. diagram (Fig. 27) and can be computed from $r$ and $Z$, the latter being determined in the usual manner from the saturation and short-circuit curves. The two methods of constructing this diagram give the same results when the value of $Z$ employed is that corresponding to the point $E_{1}$ on the saturation curve.

Thus the ampere-turn diagram is completely and easily determined by the same information required by the E.M.F. diagram; namely, the armature resistance, the saturation and short-circuit curves, and the impressed E.M.F.

In the case of the polyphase machine, $P_{2}, E_{1}$, and $r$ should designate the per-phase values.

The ampere-turn or M.M.F. diagram has several advantages over the E.M.F. diagram.

First, the substitution of an equivalent M.M.F. for the leakage impedance E.M.F., involves less error than the substitution of an equivalent E.M.F. for the armature M.M.F., because in the common type of synchronous motor the armature M.M.F. is large as compared with the leakage impedance E.M.F.

Second, in determining the excitation or phase characteristics (Fig. 79) by means of the M.M.F. diagram, $F$ is given directly in field amperes, the quantity actually measured, and $A_{1}$ is given in equivalent field amperes, for which the corresponding armature current can be taken directly from the short-circuit curve, which is usually a straight line; whereas when taken from the E.M.F. diagram the results are in terms of armature current and of $E_{2}$, the latter being a hypothetical E.M.F., which would be induced if there were no armature reaction, and assumed constant for constant excitation.

Third, the M.M.F. method gives a clearer picture of the actual phenomena. E.g., if all the constants refer to two similar machines (generator and motor) together with the connecting line, and if these machines are of the revolving field type, $R_{1}$ and $F$ are respectively the field M.M.F.'s of generator and motor, and their relative 
the results would be found to differ widely from the curves of Fig. 79, the latter being much more accurate.

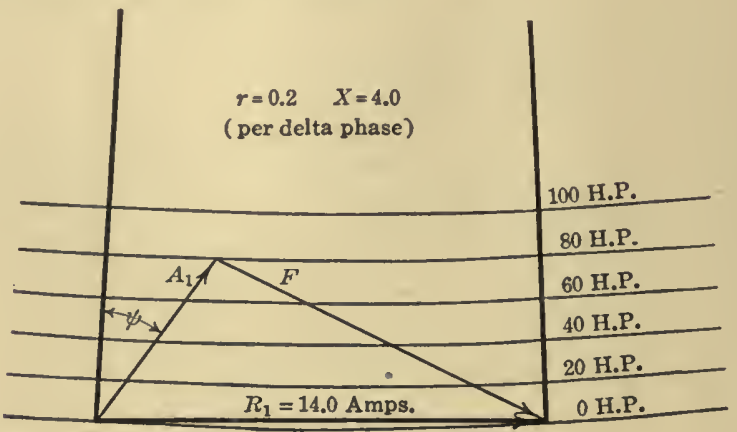

FIG. 78 .

\section{Approximate Diagram}

If the resistance $r$ be neglected in Fig. 76, $\overline{O C}$ and $\overline{A C}$ will become vertical and parallel, and the constant-power circles will degenerate into straight lines (see Fig. 8I).

Following the interpretation of Figs. 27 and $(76)$ we have, as the general interpretation of Fig. 8r:-

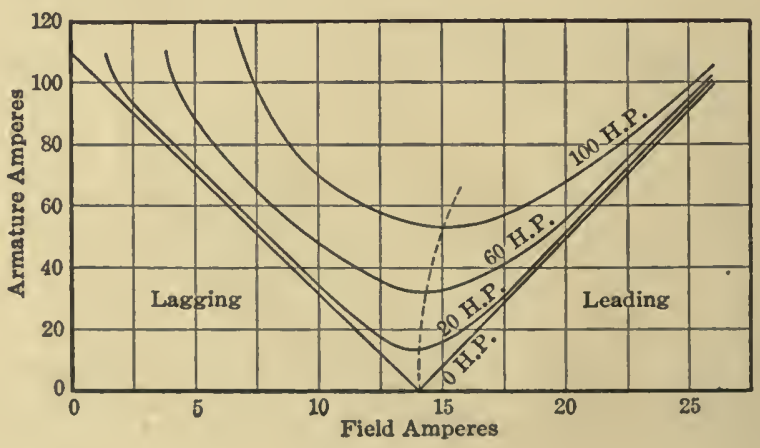

Fic. 79 .

The region to the right of the vertical line $\overline{S S}$ corresponds to unstable operation, while the region to the left corresponds to stable operation.

The region above $\overline{O D}$ and between $\overline{L L}$ and $\overline{S S}$ corresponds to 
lagging motor current, while that above the line $\overline{O D}$ and to the left of $\overline{L L}$ corresponds to leading motor current.

The region below $\overline{O D}$ and between $\overline{L L}$ and $\overline{S S}$ corresponds to leading generator current, while that below $\overline{O D}$ and to the left of $\overline{L L}$ corresponds to lagging generator current.

\section{Extreme Cases}

The assumptions (involved in the above-described diagrams) that the flux $\Phi$ has the same direction as $R$, and that constant $\Phi$ means constant $R$, irrespective of the direction or space phase of $\Phi$, become

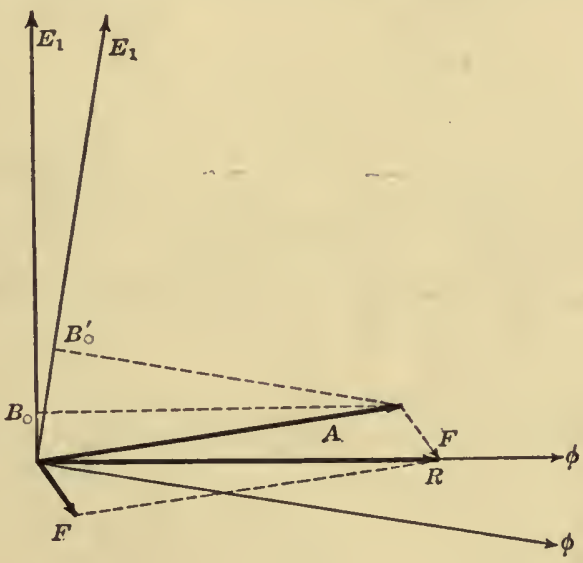

FIG. 80.

less and less warrantable as the conditions of operation depart farther and farther from the normal.

E.g., consider the case of light load and very low excitation (Fig. 8o). $\quad R$ makes such a large angle with $F$ (the axis of the field magnets) that $\Phi$ will not only be less in proportion to $R$, owing to the greater reluctance of the magnetic circuit in this direction, but it will also be pulled down more nearly into the direction of $F$, because that is the direction of minimum reluctance. Then $E_{1}\left(=E_{1}{ }^{\prime}\right)$ will also be shifted by an equal angle, since it must be in quadrature with $\Phi$. This is shown by the light lines of Fig. 80. Thus $R$ will lag behind $E_{1}$ by less than $90^{\circ}$, and if $F$ be reduced to zero, $A(=R)$ will still have a torque component and the motor will operate under very light load without any excitation. 
The physical explanation of this is that the armature current reacts upon a flux produced wholly by said armature current. - Ordinarily this self-produced flux would be perpendicular to the plane of the coil, and would therefore have no component in the plane of the coil upon which the current could react to produce a torque; but in this case the unsymmetrical reluctance of the magnetic circuit results in a flux not in the same direction as the M.M.F., and the motor will actually run without excitation at very light load. The armature current is, of course, excessive.

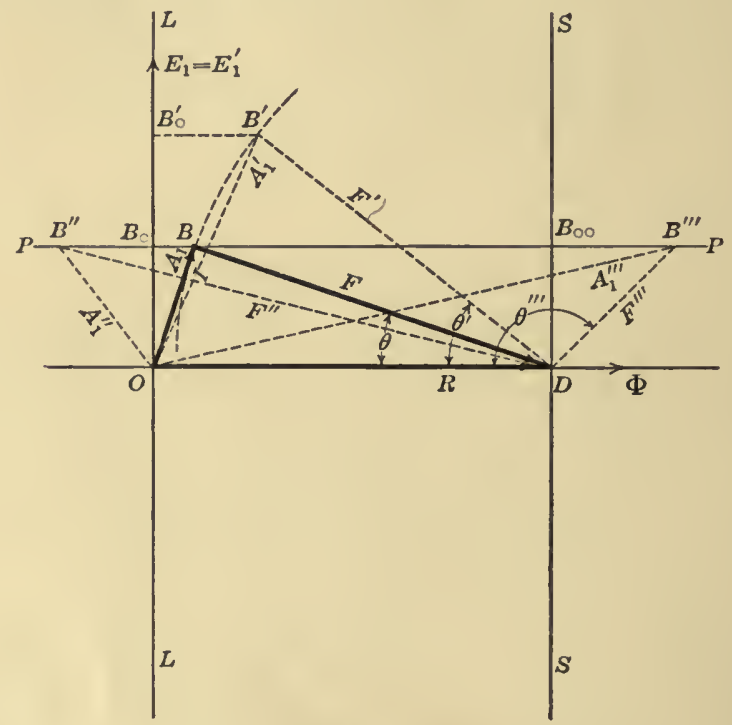

Fig. 8I.

\section{Mechanical Analogue}

The approximate diagram of Fig. 8I lends itself readily to the explanation of a very interesting mechanical analogue to the synchronous motor.

Consider two shafts, a driver and a driven, placed end to end in the same line and connected together by an elastic coupling consisting of an elastic string or band, the two ends of which are connected to two crank pins on the two adjacent ends of the shafts in question. Assume further that the tension of the elastic band is directly proportional to the distance between the two crank pins which lie in the same plane perpendicular to the shafts. In Fig. 82 is shown a 
diagrammatic end view of this coupling: the point $D$ is the center of the shaft; the line $\overline{D O}$ is the radius of the driving crank pin and $\overline{D B}$ that of the driven crank pin; $\overline{O B}$ is thus the distance between the two crank pins, to which the tension of the elastic band is proportional; the lever arm of the tension about the center of the shafts is $\overline{D C}=\overline{D B} \cos \phi$, and the corresponding turning moment transmitted from driver to driven is thus proportional to $\overline{D C} \times \overline{O B}$. But $\overline{D C}=\overline{D O} \cos \psi$, and the torque or turning moment is $T=\overline{O B} \times \overline{D O}$ cos $\psi$; or since. $\overline{O B} \cos \psi=\overline{O B}_{0}, T=\overline{O B}_{0} \times \overline{O D}$; i.e., the torque is proportional to the area of the rectangle $\overline{D O B}_{0} . \quad O B_{0}$ is the tangential

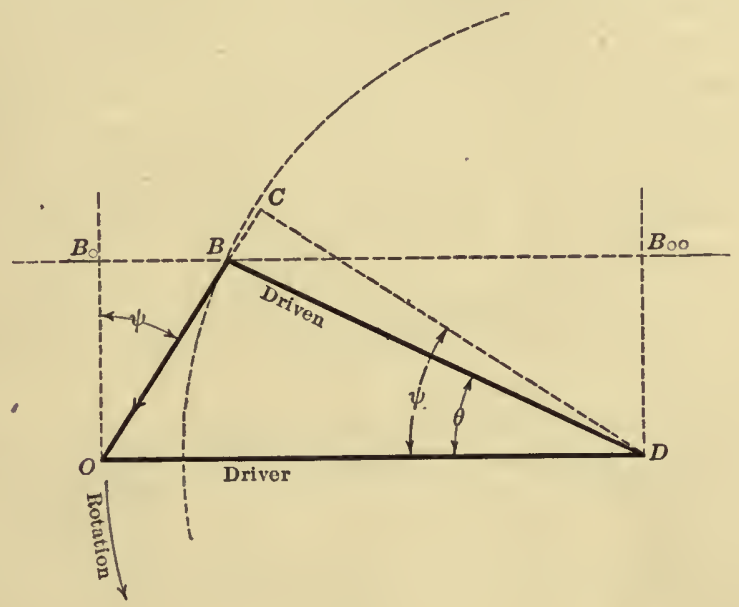

FIG. 82 .

component of the tension $\overline{O B}$, and $\overline{O D}$ is the corresponding lever arm.

Consider now the operation of this coupling for given crank pin radii and a given stiffness of the elastic band $\overline{O B}$. At no load or zero torque the crank $\overline{D B}$ will be pulled ahead into line with $\overline{D O}$, the tangential component of the tension $\overline{O B}$ will be zero, the angle $\theta$ will be zero, $B_{0}$ will coincide with $O$, and the rectangle $D O B_{0}$ will collapse into a line according to the above-indicated relation between the torque and the area of this rectangle. If now the load torque be increased, the point $B$ will fall back to the right, and the angle $\theta$ will increase until $\overline{O B}_{0}$, the tangential crank effort, has attained the the value imposed by the load. A further increase in the load torque will be accompanied by a further increase in $\theta$ and in $\overline{O B}_{0}$; but it 
will be observed that there is a limit to the possible increase of $\overrightarrow{O B}_{0}$, namely, when $\theta$ is $90^{\circ}$ and $\overrightarrow{O B}_{0}=\overline{D B}$, beyond which the tangential crank effort $\overline{O B}_{0}$ decreases, to zero when $\theta=180^{\circ}$, to a negative maximum when $\theta=270^{\circ}$, and back to zero again when $\theta=360^{\circ}$ or $\circ^{\circ}$. Thus although the tension $\overrightarrow{O B}$ goes on increasing after $\theta$ has passed the $90^{\circ}$ point, the lever arm decreases more rapidly than the tension increases, i.e., the tangential component $\left(\overline{O B}_{0}\right)$ of the tension decreases.

Assume now that with constant load-torque the driven crank pin radius is altered. The tangential crank effort, $\overline{O B}_{0}$, must remain the same, and the driven crank pin $B$ must lie somewhere on the line $\bar{B}_{0} \bar{B}_{00}$. Thus for a given driver crank radius, the angle $\theta$ and the tension $\overline{O B}$, depend upon the torque demanded by the load (which is proportional to $\overline{O B}_{0}$ ), and upon the driven crank pin radius $\overline{D B}$. For each value of the load torque and of $\overline{O B}_{0}$, the tension will be a minimum for that value of the driven crank pin radius $\overline{D B}$, which causes $B$ to fall at $B_{0}$. It is obvious that this particular value of $\overline{D B}$ varies with the load.

If now the driver crank pin radius $\overline{D O}$ be increased, the value of $\overline{O B}_{0}$ for a given load will be decreased and vice versa, since their product must be constant.

It has been assumed thus far that a fixed relation exists between the crank pin displacement $\overline{O B}$ and the coupling tension; but while this is true for a given case, it is evident that a different elastic band or spring could be substituted which would have a different elastic coefficient, and with which the angle $\theta$ would be quite different for the same load.

The angle $\theta$ will be hereafter referred to as the coupling angle. It obviously depends upon the load torque, the two crank pin radii, and the elastic coefficient of the coupling.

Let $p=$ tension in pounds $=k \times \overline{O B}$, where $k$ is the coefficient of the elastic band. Then if $\overrightarrow{D O}$ and $\overrightarrow{D B}$ are measured in feet, the torque in pound-feet will be

$$
T=k \times \overline{O B}_{0} \times \overline{D O}=k \times \overline{O B} \cos \psi \times \overline{D O}=k \times \overline{D B} \sin \theta \times \overline{D O} .
$$

Thus for any given value of the crank pin radii, the torque varies as the $\sin 0$.

A comparison of the diagrams of Figs. $8 \mathrm{I}$ and 82 will show their exact mechanical equivalence. In both cases the torque is proportional to $\overline{O B}_{0}$ for a given value of $\overline{O D}$; therefore the relation of the torque to $\theta$ and to $\overline{D B}$ is exactly the same in the two cases. In 
Fig. 8I $\overline{O B}$ is a measure of the armature M.M.F., i.e., the strength of the armature considered as an electromagnet lying across the magnetic field whose flux is $\Phi$ and whose M.M.F. is $R$ (see Fig. 83). The resulting torque is then proportional to $A_{1} \Phi \cos \psi$ or approximately proportional to $A_{1} R \cos \psi$; but in this approximate analysis, $\Phi$ and therefore $R$ is determined by the impressed E.M.F., and is in any given case a rough measure of the exciting M.M.F of the generator which supplies the power, while $F$ is the exciting M.M.F. of the synchronous motor.

Thus the two crank pin radii correspond roughly to the exciting M.M.F.'s of the generator (driver) and the motor (driven), the tension $\overline{O B}$ corresponds to the armature M.M.F., the angle $\psi$ to the phase difference between current and E.M.F. or to the angle by which the armature M.M.F. differs from its position of maximum

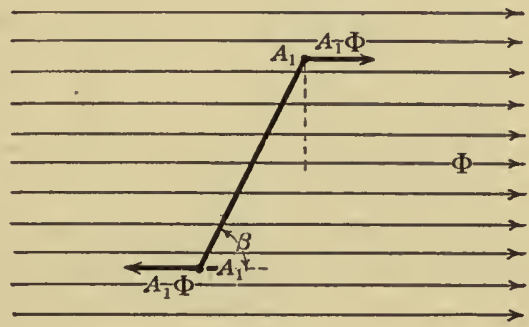

Fig. 83.

torque-producing effect, and the coupling angle $\theta$ roughly to the mechanical phase difference (measured in electrical degrees) between the revolving parts of the generator and motor.

The electrical angular velocity of the motor is the same as that of the generator, but their mechanical angular velocities are inversely as their numbers of poles; while in the mechanical analogue the angular velocities of the two parts of the coupling must be the same.

In order to see what determines the stiffness of the electromagnetic coupling between alternator and synchronous motor, it is necessary to remember that the torque is strictly proportional to the product of the flux $\Phi$ and that component of the armature M.M.F. in quadrature with $\Phi$, and that for a given value of $\Phi$ the necessary M.M.F. $R$ will depend upon the reluctance of the magnetic circuit; e.g., if a synchronous motor has its pole faces bored back so as to increase the reluctance of the magnetic circuit, a larger resultant 
excitation $R$ must be supplied in order to produce the same flux $\Phi$ made necessary by the same impressed E.M.F.; but with this same flux, the same load will require the same torque component $\overline{O B}_{0}$ of the armature M.M.F. $A$. Thus although $R$ and $F$ have increased, $\overline{O B}_{0}$ is unchanged, and the angle $\theta$ reduced, for the same ratio of $F$ to $R$. This decrease of the angle $\theta$ for a given torque corresponds to a stiffening of the elastic band in the mechanical coupling, and increases the break-down torque (when $\theta=90^{\circ}$ ). In fact, the abovedescribed change in the synchronous motor is commonly called a stiffening of the field, in that the latter is then less distorted by a given armature M.M.F.

It will be observed in this connection that every synchronous motor has a natural period of oscillation about its mean running position, which depends upon the moment of inertia of the revolving part and the stiffness of the electromagnetic coupling. These oscillations correspond to a variation of the coupling angle $\theta$ about its mean value, and a pulsation of power-flow. If the angular velocity of the supply alternator should oscillate about a mean value, the resulting oscillation transmitted to the synchronous motor through the electromagnetic coupling would depend upon the amplitude and frequency of the impressed oscillation, upon the moment of inertia of the revolving part of the motor, and upon the stiffness of the coupling. If the impressed oscillation should have the same frequency as that of the freely oscillating motor, the latter would tend to increase its amplitude of oscillation until it would swing past the point of maximum torque $\left(\theta=90^{\circ}\right)$ and break down. If, on the other hand, the frequencies were widely different, the amplitude of the transmitted oscillation would be greater, the stiffer the coupling and the less the moment of inertia of the revolving part.

These phenomena are usually covered by the term "hunting" or "phase swinging," a quantitative analysis of which is given in Chapter IV.

\section{Length of Air-Gap}

It will be interesting here to review the relative merits of high and low synchronous impedance in a synchronous motor; i.e., of a soft and a stiff electromagnetic coupling, or what is equivalent, the relative merits of a short and a long air-gap.

Take first a system in which there is a considerable pulsation of 
frequency due to the non-uniform crank effort of a single cylinder steam-engine which drives the supply alternator.

If the impedance (including generator, motor and line) be very low, i.e., if the air-gap be long and the coupling stiff, the motor speed will follow closely the frequency pulsation. There is thus a pulsation strain on the coupling accompanied by a large pulsation of power and current. If the motor is large enough as compared with the alternator, the stiff coupling will have the effect of adding flywheel capacity to the alternator and will tend to reduce the frequency pulsation, but at the expense of heavy current and power pulsations. If, on the other hand, the impedance be large, the frequency pulsations will be partly absorbed in the soft coupling, the motor will not follow closely the frequency pulsations, and there will be much less pulsation of power and of current. There is, however, an obvious limit to the desirable softness of coupling, namely, that beyond which there is danger of a breakdown of the motor because of the soft coupling.

There is also another objection to the soft coupling, which will appear from an inspection of the E.M.F. diagram (Fig. 27). If the reactance be high, the coupling angle will be relatively large for a given power, and there will be a large variation in the power factor of the motor from no load to full load under constant excitation, or a frequent adjustment of excitation will be demanded in order to maintain a constant power factor.

When polyphase synchronous motors or synchronous converters are started by the induction motor action of the damping coils, or by hysteresis and eddy current torque, it is desirable to have as short an air-gap as possible in order to keep the necessary starting current within limits.

The choice of air-gap is thus a compromise and depends somewhat upon the particular service demanded of the motor in question, and upon the nature of the system on which it operates. The gap should preferably be not as long as demanded by good regulation in the synchronous alternator, nor as short as demanded by high-power factor in the induction motor or induction generator. On this point there is considerable difference in practice, particularly in the case of synchronous converters. 


\section{CHAPTER VIII}

\section{GENERALIZATION OF DIAGRAM FOR COUPLED SYNCHRONOUS MACHINES}

THE diagram represented in Fig. 27 (page 44), which is based upon Joubert's theory that the arnature-reaction of the alternator employed can be adequately explained by supposing the presence in the armature of a simple mean synchronous reactance, can be retained to advantage for certain purposes even in the case of the theory of "two reactions," developed by the author in Part III, Chapter I, for alternators with saturated magnetic fields. It may, indeed, be noted that the impedance $Z$, which enters into the expression for the vector, $\overline{A_{1} A_{2}}$, is still a constant of the same character as before, though it now represents the impedance of the transverse reaction, which may be symbolized by $Z_{l}$, when we take

$$
Z_{t}=\sqrt{r^{2}+\omega^{2} L_{t}^{2}}
$$

We will first suppose the case of two alternators which comply with Joubert's law (i.e., which have a constant synchronous reactance). The clearest form of electrical transmission diagram will be obtained by first combining the diagrams shown in Fig. 27 (page 44) and in Fig. 43 (page 86). All that is necessary is to point off along $O A$ (Fig. 84) a distance proportional to the voltage $(U)$ at the terminals of the two alternators, these being assumed to be near each other. (If. they are far apart the inpedance of each one is to be increased fictitiously by an amount equal to half the impedance of the line by which they are coupled together). The vectors of the e.m.f.'s of these alternators are then drawn in proper magnitude and phase. (The alternators may be unlike provided they both have the same characteristic ratio $\frac{\omega) L_{t}}{r}=\tan \gamma$ ). We then draw the line of zero 
phase-angle, $A N$, lagging by the angle, $\gamma$, with respect to $U$, and point off, at $N$, the center of the circles of constant internal power, which will be the same for the generator as for the motor. The three points $A_{1} A A_{2}$ are then joined by a straight line, $A_{1} A_{2}$, which represents twice the product $Z_{t} I$, of the impedance $Z_{t}$ by the current, $I$, that is to say twice the impedance e.m.f. of the circuit. In reality the e.m.f. $U$ may be considered as being that of a "line" absorbing the energy of the generator and supplying the

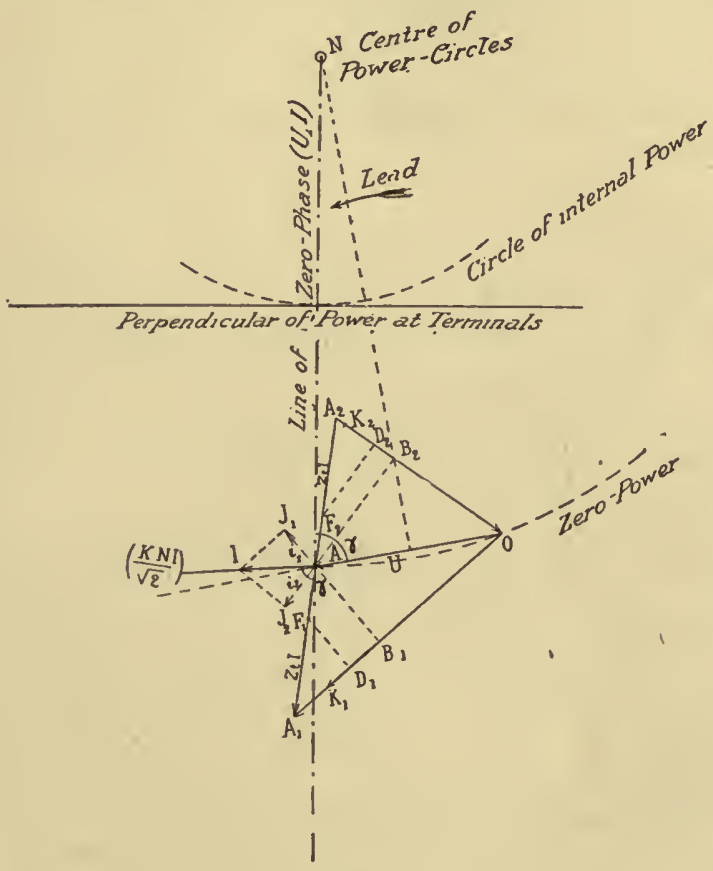

FIG. 84 .

same energy to the motor. We thus introduce a "fictitious" line which does not change the phenomena in any way, but which has the advantage of enabling the diagram to be generalized completely. The current $I$ passing between the two alternators is equal to $\frac{A A_{1}}{Z_{l}}$ and it lags behind $A A_{1}$ by the angle $\gamma$.

In the "two-reactions" theory, this diagram is modified in the manner now to be explained. The phases of the currents are 
still deduced from those of the e.m.f.'s by means of the internal resistance $r$ and an inductance; but that inductance is then the total transverse inductance $L_{t}=l_{t}+s$. The sides, $A_{2} A$ and $A A_{1}$, of the triangles will therefore represent $Z_{t} I$, where $Z_{i}$ symbolizes the impedance which corresponds to the transverse inductance, where $I$ represents the current, and where $\tan \gamma=\frac{\omega L_{t}}{r}$. But $O A_{1}$ and $O A_{2}$ no longer represent the internal e.m.f.'s except in regard to their phases. To represent them in magnitude as well as in phase, the diagrams shown in Fig. 85 must be constructed by reference to the characteristic curves for these two alternators, which curves, for the sake of simplicity, are here supposed to be the same for both machines.

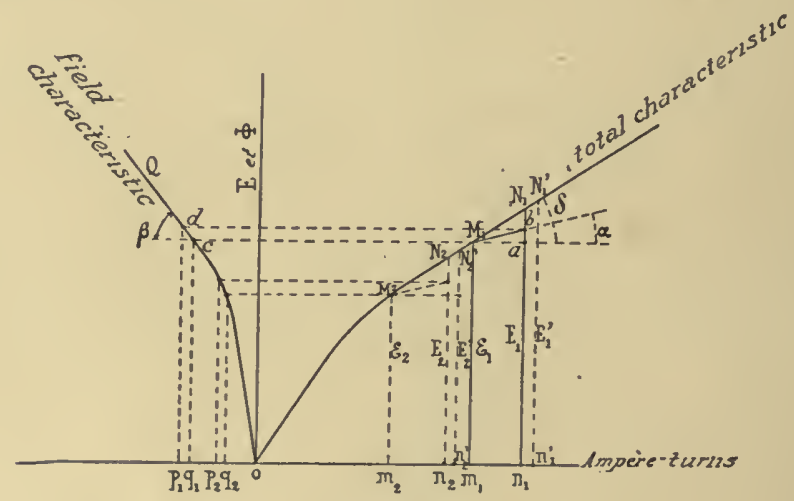

FIG. 85 .

Let $O M_{1} N_{1}$ be the total excitation characteristic or saturation curve obtained by plotting the excitation ampere-turns as abscissas and the induced e.m.f.'s at no load as ordinates. Let $\frac{K N I}{\sqrt{2}}=$ the armature ampere-turns produced by an effective current $I$ in the armature (with $N$ peripheral conductors per pair of poles; and let us select, for Fig. 84, a scale such that the current-vector $A I$ will also represent these armature ampere-turns according to the scale of abscissas in Fig. 85. We then draw $A B_{1}$ and $A B_{2}$, perpendicular respectively to $O A_{1}$ and $O A_{2}$ (in Fig. 84); and we point off $A F_{1}$ and $A F_{2}$ both equal to $\omega S I$, and also draw $F_{1} D_{1}$ and $F_{2} D_{2}$, perpendicular respectively to $O A_{1}$ and $O A_{2}$. The vectors $O D_{1}$ and $O D_{2}$ represent the 
internal e.m.f.'s generated in the two alternators by the direct flux, i.e., the flux along the field axis. Let $\delta_{1}$ and $\delta_{2}$ represent these two e.m.f.'s, and let them be drawn as ordinates on the total characteristic curve (Fig. 85). The corresponding abscissas $o m_{1}$, and $o m_{2}$, represent the excitation ampere-turns necessary to generate these e.m.f.'s at no load. We add to them, respectively, the counter ampere-turns of the armature, $m_{1} n_{1}$ and $m_{2} n_{2}$, obtained, respectively, oy projecting (Fig. ${ }^{84}$ ) the vector $A I$ on the perpendiculars to $O A_{1}$ and $O A_{2}$, since these projections represent the reactive components of the armature ampere-turns. They are

$$
\frac{K N_{1} J_{1}}{\sqrt{2}} \text { and } \frac{K N_{2} J_{2}}{\sqrt{2}}
$$

in which $N_{1}$ and $N_{2}$ represent the numbers of peripheral armatureconductors per pair of poles in the two machines, and $K$ is the utilization-coefficient for the particular winding. We thus obtain the e.m.f.'s $E_{1}$ and $E_{2}$; but these are not yet altogether exact, because we have neglected the small increases of excitation which are necessary to compensate for the increase in saturation of the field magnet cores resulting from the increase in magnetic leakage between the polepieces. The correction necessary is very easily made, if the permeance $B_{f}$, of the leakage path is known and if the excitation-characteristic of the magnetic field cores alone $(O Q)$ has been drawn, as shown in Fig. 85 , by plotting it reversed, to the left of the axis of ordinates, taking the excitation ampere-turns as abscissas and the magnetic flux through the magnets and yoke as ordinates. This characteristic curve should be drawn according to a scale of ordinates such that the magnetic fluxes may be represented by the electromotive forces which they would generate in the armature if they were to thread through it. Let $\beta$ be the angle of the tangent to the curve $O Q$ at the point $c$. Let us now draw at $M_{1}$ an angle $b M_{1} a=\alpha$, whose tangent represents the permeance $B_{f}$ of the magnetic leakage path. Then the vertical segment $a b$, intercepted on the line $N_{1} n_{1}$, represents the additional magnetic leakage flux. Let us draw two horizontal lines $a c$ and $b d$, and take their intersection with the fieldcharacteristic (at the left in Fig. 85), and let us then find the corresponding abscissas, $p_{1} q_{1}$. The segment $p_{1} q_{1}=\frac{\tan \alpha}{\tan \beta} m_{1} n_{1}$ represents and measures the supplementary ampere-turns required. Let us 
take $n_{1} n_{1}^{\prime}=p_{1} q_{1} ;$ and $m_{1} n_{1}^{\prime}$ represents and measures the total ampere-turns made necessary by the armature-current. In like manner, $m_{2} n_{2}^{\prime}$ represents the corresponding ampere-turns required for the motor $O A_{2}$ at the output indicated.

To the two values $o n_{1}^{\prime}$, and $o n_{2}^{\prime}$, of the total ampere-turns, correspond electromotive forces $E^{\prime}$, and equal, respectively, to $N_{1}{ }^{\prime} n_{1}^{\prime}$ and $N_{2}^{\prime} n_{2}^{\prime}$, on the total characteristic. These e.m.f.'s (which are those that would appear if the current $I$ ceased suddenly to pass through the alternators), correspond, in the present case, to what we term internal electromotive forces in the case of Joubert's theory. By measuring off (Fig. 84) from $O$, along $O A_{2}$ and $O A_{1}$, distances $O K_{1}$ and $O K_{2}$, respectively equal to these e.m.f.'s, we obtain two vectors which replace, in the "two-reactions" theory, the vectors $O A_{1}$ and $O A_{2}$ in the diagram obtained with Joubert's theory.

It is seen that, in consequence of saturation, these vectors are, in general, shorter than $O A_{1}$ and $O A_{2}$; but this is not a necessary result, because it is possible to design an alternator in which the transverse reaction would be very small, by reducing the width of the pole-pieces, and which could, consequently, lead to a length for $O A_{1}$ less than $O K_{1}$, at least so long as the saturation of the fieldmagnets is low.

For the sake of simplicity $L_{t}$ has been assumed constant at all loads, because the influence of the air-path is generally preponderating in the transverse magnetic circuit, contrary to what is true for the principal circuit; but those who may wish greater refinement can take into account the slight variations of $L_{t}$ with the load-conditions of the alternator, and they can also replace the perpendicular straight lines $A B_{1}$ and $A B_{2}$ by curves comprised between these straight lines and segments of circles. In this way allowance can be made for the fact that, in certain cases, the transverse reaction may not raise the voltage at the terminals as much as when the pole-pieces are wide. This complication of the diagram does not materially increase its precision and it is therefore preferable to use the simpler theoretical diagram.

The circles of constant power of the old diagram are to be replaced here by lines, which are drawn by points. To obtain these lines, the values of $E_{2}^{\prime}$ are drawn or determined for a large number of positions of $A_{2}$ and for the corresponding power-outputs, and each power-value is indicated near the point $A_{2}$ corresponding to it. When the entire diagram has been in this way covered by a sufficiently 
numerous series of points, these may be considered as being "levels" on a topographical plan, and lines connecting together the points at the same "levels" can be drawn like the "contour" lines on topographical plans. Between any two such lines of constant power, other lines of constant power of intermediate value can be drawn by interpolation in the well-known way. 


\section{PART II}

\section{GENERAL DIAGRAMS DEDUCED FROM THE DIAGRAM FOR SYNCHRONOUS MOTORS}

\section{CHAPTER I}

\section{GENERAL DIAGRAMS DEDUCED FROM THE DIAGRAM FOR SYNCHRONOUS MOTORS}

Introduction. The author presented, at the Electrical Congress in Paris, in I900, a paper on The Graphical Theory of Rotary Converter Regulation, the object of which was to set forth a purely graphical, but nevertheless complete, method of treating this complicated though interesting question, and to show, at the same time, that the solution of the problems involved could be reduced to the solution previously worked out for synchronous motors, by the author, and could be reconciled with his "theory of two armature-reactions in alternators."

In accordance with that theory, the rotary converter can, in effect, be treated as a simple particular case of the synchronous motor, namely, as a synchronous motor having no transverse reactions; and the vectors obtained for synchronous motors are rendered applicable to rotary converters by merely suppressing the transverse reaction vector.

It is thus, seen that this theory contains nothing artificial, but that, on the contrary, it is both general and homogeneous to a high degree.

Notation. The notation used may be substantially the same as for synchronous motors; but the meaning of some of the subscripts must be changed somewhat to take into account the fact that the armature of the rotary converter receives currents of two kinds, primary 
and secondary. (The primary and secondary windings can be different.) The notation used will be the following:

$T=$ periodic time (duration of one period), in seconds;

$\omega=\frac{2 \pi}{T}=$ frequency, or speed of pulsation of the current;

$R=$ total resistance of circuit (generator, line, and primary winding of converter); ${ }^{1}$

$l=$ cumulative self-induction of generator and line;

$s=$ self-induction of converter due to magnetic leakage of armature;

$L=$ self-induction added in the form of reactance-coils;

$X=\omega(L+l+s)=$ total reactance of alternating-current circuit;

$Z=\sqrt{R^{2}+X^{2}}=$ total impedance corresponding;

$E=$ effective E.M.F. (being the E.M.F. supplied by the line or that induced in the generator, in the case of a simple transmission circuit). This E.M.F. is to be considered always constant unless otherwise stated;

$q=$ number of phases of the primary current;

$\varepsilon=$ effective primary (alternating) E.M.F. of the converter;

$\frac{\varepsilon}{\xi}=E_{2}=$ secondary or induced (D.C.) E.M.F.;

$\xi=$ ratio of transformation of the converter;

$I=$ effective value of one of the primary (alternating) currents;

$I_{w}$, or $J$, =active component of that current;

$I_{d}=$ reactive component of that current;

$\phi=$ phase-angle of lag corresponding to $\left(\tan \phi=\frac{I_{d}}{I_{w}}\right)$;

$\theta=$ phase-angle between the E.M.F.'s $\varepsilon$ and $E$;

$I_{2}=$ secondary (direct) current, proportional to $J$ less the losses;

$N=$ number of peripheral primary conductors per magnetic field;

$K=$ reduction-coefficient for primary winding;

$N_{2}=$ number of peripheral secondary conductors per magnetic field;

$A=$ ampere-turns of (shunt) field-coils;

$n=$ number of exciting turns of series-winding;

$r=$ resistance per turn of shunt-winding;

$j_{0}=$ active current when the load is zero;

$i_{0}=$ reactive current when the load is zero.

The resistance of the line and generator are negligible in the case of a rotary converter supplied with current from a large distribution-system. 


\section{GENERAL DIAGRAMS FOR SYNCHRONOUS MOTORS}

Generalities. Reduction of all Armature-Reactions to the Single Direct Reaction. The inducing field is the resultant of three magnetomotive forces (Fig. I):

I. The inducing ampere-turns of the exciting current;

2. The ampere-turns which are reactive with respect to the E.M.F. and which act in the same way as the preceding;

3. The difference between the active ampere-turns and the secondary ampere-turns produced by the direct current. These two kinds of ampere-turns tend to produce fields which are diametrically opposed to each other and which make a phaseangle of $\frac{\pi}{2}$ with respect to the exciting field.

Practically, the theory is greatly simplified by the fact that the active ampere-turns and the direct-current secondary ampere-turns are substantially equal to each other at all loads.

On the one hand, the winding being a direct-current winding, the average number of active ampere-turns will be given by the formula,

$$
K \frac{N}{2} I_{w} \sqrt{2}, \quad . \quad . \quad . \quad . \quad . \quad . \quad .
$$

in which $N=$ number of wires;

$I_{w}=$ açtive component of primary alternating current;

$K=$ reduction-coefficient due to the overlapping of the windings, for an infinitely large number of slots.

The author has published some average values for this coefficient. It should, preferably, be determined from existing machines.

On the other hand, the average number of ampere-turns due to the direct current, $I_{2}$, produced by the converter, is equal to the mean value,

$$
\frac{\mathrm{I}}{2} \frac{N}{2}\left(\frac{I_{2}}{2}\right)
$$

Dividing $(b)$ by $(a)$ we have the ratio of the secondary and primary ampere-turns:

$$
\zeta=\frac{\mathrm{I}}{4 K \vee 2}=\frac{I_{2}}{I_{w}}
$$

Whatever may be the number of phases, $q$, if the efficiency be assumed equal to unity, which makes the primary and secondary 
watts equal, the induced E.M.F., as a function of the reductioncoefficient $k$, due to overlapping, may be expressed thus:

$$
I_{w} \frac{2 \pi k}{{ }_{2} \sqrt{2}} \frac{N \Phi}{T}=I_{2} \frac{N \Phi}{T}, \ldots . . . \quad . \quad .
$$

in which $T=$ the periodic time, and $\Phi=$ the magnetic flux.

From this, we have

$$
\frac{I_{2}}{I_{w}}=\frac{\pi k}{\sqrt{2}} . . . . . . . . . .
$$

Substituting in $(c)$ we have

$$
\zeta=\frac{I}{4 K \sqrt{2}}=\frac{\pi k}{\sqrt{2}}=\frac{\pi k}{8 K}
$$

I have shown elsewhere (see Properties of Revolving Magnetic Fields, Eclairage Electrique, 1895$)$ that the ratio $\frac{k}{K}$ is substantially equal to $\left(\frac{\pi}{2}\right)^{2}$. We therefore have

$$
\zeta=\frac{\pi}{8}\left(\frac{\pi}{2}\right)^{2}=\frac{\pi^{3}}{32}=\frac{30.935}{3^{2}}=0.969 .
$$

This close approach of the value of $\zeta$ to unity shows that the transverse reaction is practically negligible in comparison with the direct reaction. Hence rotary converters may be treated, practically, as if there were no transverse reaction in them.

If the internal losses are taken into account, the secondary (output) watts will be from to to I5 per cent less than the primary (input) watts; and the

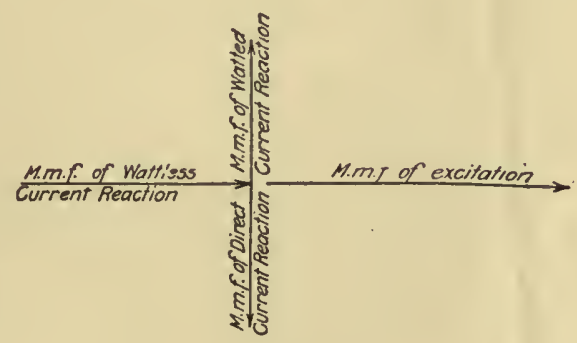

FIG. I. ratio $\zeta$ will be still less near unity. Nevertheless it is found that:

I. The direct-current E.M.F. undergoes no modification in consequence of the transverse reaction, because the brushes always remain 


\section{GENERAL DIAGRAMS FOR SYNCHRONOUS MOTORS}

absolutely at the neutral point, and, consequently, only the direct flux is cut by the armature conductors;

2. The alternating current E.M.F. is affected only to a slight extent, because the field resulting from the active current $I_{w}$, and from a direct current $I_{2}$, which is Io to 15 per cent weaker, constitutes only Io to 15 per cent of the transverse reaction field of the direct-current armature, which field, as a rule, would not exceed half the direct field. But, even assuming that this resultant transverse field may amount to 20 per cent of the direct field, the alternating E.M.F. would be thereby increased only in the ratio,

$$
\frac{\sqrt{\mathrm{I}+(0.20)^{2}}}{\mathrm{I}}=\mathrm{I} .02 \text {, }
$$

or, only about two per cent, which is negligible. We are therefore warranted in treating the rotary converter as a synchronous motor having no transverse reaction, as has been done by other authors. There might possibly be an exception in the case of rotary converters having no field-excitation; but these are used very little, and their theory, in any case, can be established much more easily by considering them as transformers.

As in the case of every alternator, the armature, in a rotary converter, is subject to magnetic leakage, that is to say a portion of the magnetic flux produced by the alternating currents in the armature winding is shunted by the air ur by the armature-teeth. This leakage is offset in part by the leakage which the secondary current tends to produce, in the contrary direction (and which offsets, especially, the effect of the active current); hence, the resultant leakage is much smaller than in a synchronous motor. Nevertheless, in order to make the theory general, we will suppose that this magnetic leakage exists; and it will be represented, as usual, by an inductance, $s$, giving rise to a reactance, $\omega$. .

Factors Determining the Practical Conditions of Operations. It is very important to note that the operation of rotary converters does not depend on the characteristics of the machine only, but also depends on the constants of the electric supply-circuit. It is, in fact, the self-induction of the supply-circuit and the difference between its E.M.F. and the E.M.F. induced in the converter, which govern the ratio of reactive to active currents.

Every active current lagging behind the E.M.F. produces a demagnetizing effect, whereas every reactive current leading the 
E.M.F. produces a magnetizing effect. The variations in the conditions of the external circuit, therefore, constitute an indirect process for making the field-excitation of the rotary vary, though this variation is often contrary to what is wanted.

I. CONDITIONS OF ELECTRIC-CURRENT SUPPLY TO ROTARY CONVERTERS

From what precedes, it is important to know, quite precisely and quantitatively, the manner in which the reactive current varies with the conditions of the current-supply to the converters, especially with

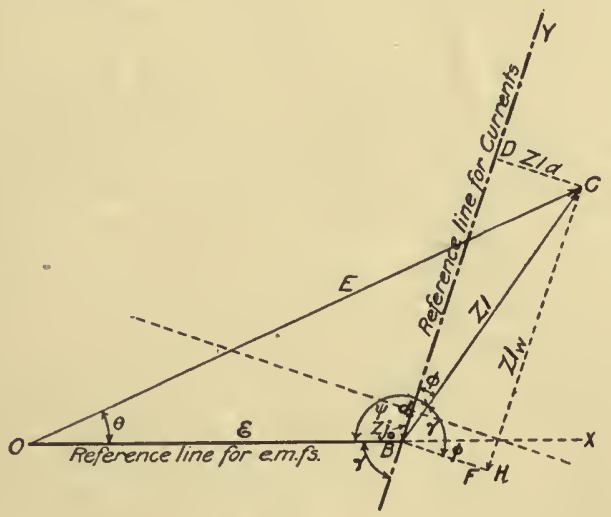

Fig. 2.

the constants of the circuit and with the impressed E.M.F. This question will now be discussed.

Fundamental Diagram. We can start from the usual diagrams for alternators, made with two scales, one for currents, and the other for E.M.F.'s.

Assuming $E_{2}=$ the secondary (D.C.) voltage at the terminals, let $\varepsilon=O B$ (in Fig. 2) represent the primary voltage, which is substantially proportional to $E_{2}$, owing to the low internal resistance of the armature.

I.et $E=O C=$ the E.M.F. of the alternator supplying current to the converter (or the voltage of the distribution-system, according to the case). Unless otherwise stated $O C$ will be assumed constant at all loads. 


\section{GENERAL DIAGRAMS FOR SYNCHRONOUS MOTORS}

Let $R=$ resistance of primary circuit of converter;

$X=\omega\left(I_{-}+l+s\right)=$ reactance of same;

$Z=\sqrt{R^{2}+X^{2}}=$ impedance of same;

$\tan \gamma=\frac{X}{R}=$ reactance-coefficient;

$\omega=\frac{2 \pi}{T}=$ frequency.

The "primary" circuit in this case includes the armalure. The reactance constant $X$ must therefore include the following: The reactance $(\omega L)$ of the reactance-coils connected in series with the converter; also the reactance $(\omega l)$ of the alternator and of the line if the current is supplied directly by an alternator, and not by a distribution-system; and, finally, the reactance $(\omega s)$ corresponding to the effect of the magnetic leakage at the armature itself.

For each value, $\theta$, of the phase-angle between the E.M.F.'s $E$ and $\varepsilon$, the third side, $B C$, of the triangle $C O B$, represents the resultant E.M.F. of $E$ and $\varepsilon$, which is equal to the product of the impedance $Z$ by the primary alternating current $I$ corresponding to and passing through this impedance. Constructing the triangle $C B H$, on $B C$ as the hypothenuse, with the angle $r$ at $B$ we will have

$$
B H=X I \quad \text { and } \quad H C=R I .
$$

In the diagrams $\phi$ will always represent the angle of lag of the current behind the voltage at the terminals.

In order that the vector $B C$ may measure the current $I$ itself, both in magnitude and in phase, it is only necessary to take, for the amperes, a scale which is $Z$ times greater than for the volts, and to take, as the origin of phase-angles $(\phi)$, a reference line $B D Y$, making, with the line $O X$, the angle $\gamma$ defined hereinabove. The angle $D B C$ then measures a phase-angle $(\phi)$, representing a "lag" if it be to the right of $B D$, and a "lead" if it be to the left. We therefore count the reactive current, $I_{d}$, as positive on the right-hand side of $D$ and as negative on the left-hand side.

If, from $C$ (Fig. 3) a perpendicular $C D$ be drawn to the line of reference $B Y$, the segments $C D$ and $B D$ will measure the reactive and active currents for each position of $C$. The active current $I_{w}$ can, itself, be considered as equal to the sum of two parts. One of these parts $\left(j_{0}\right)$, represented by the segment $B d$, is practically constant and 
corresponds to the approximately constant loss produced by the " no-load" active current, in consequence of friction, resistance, hysteresis, and eddy currents. The part $j_{0}$ will be called the "active current for zero-load." The other part, represented by $D d$, corresponds to the energy of the useful secondary current, and which is proportional to the said current.

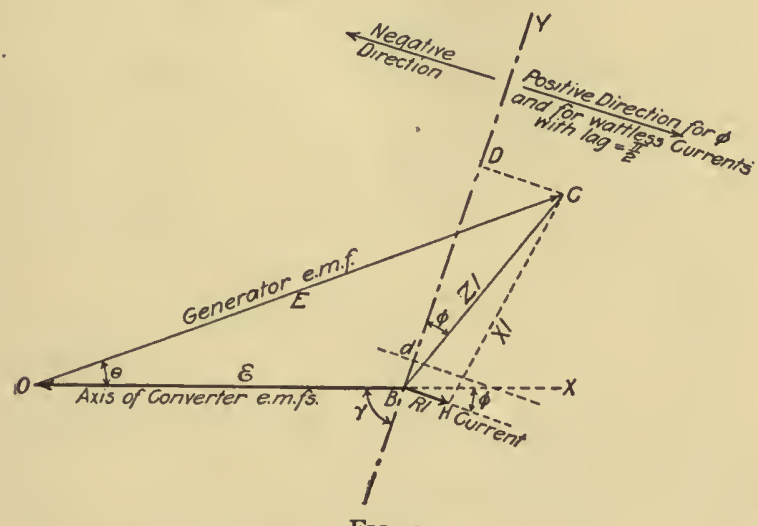

FIG. 3 .

Fundamental Equation. The fundamental equation connecting $\varepsilon, E$, and the active and reactive currents can be written directly from Fig. 2. The triangle $O B C$ gives, in fact,

$$
\left.\begin{array}{rl}
E^{2} & =\varepsilon^{2}+(Z I)^{2}-2 \varepsilon(Z I) \cos \psi \\
& =\varepsilon^{2}+(Z I)^{2}+2 \varepsilon(-Z I \cos \psi)
\end{array}\right\}
$$

In this case we have

$$
\begin{aligned}
-(Z I) \cos \psi & =\text { projection of } B C \text { on } O X \\
& =\text { (proj. of } B D+\text { proj. of } D C) \text { on } O X \\
& =(\text { proj. of } C H+\text { proj. of } B H) \text { on } O X \\
& =Z I_{w} \cos \gamma+Z I_{d} \cos \phi \\
& =Z I_{w} \cos \gamma+Z I_{d} \sin \gamma .
\end{aligned}
$$

Substituting this value, and remembering that $I^{2}=I_{w}{ }^{2}+I_{d}^{2}$, we have, from $(a)$,

$$
E^{2}=\varepsilon^{2}+Z^{2}\left(I_{w}^{2}+I_{d}^{2}\right)+2 \varepsilon Z\left(I_{w} \cos \gamma+I_{d} \sin \gamma\right) . .
$$


We also find (from the triangle $C B H$, in Fig. 3),

$$
\begin{aligned}
& Z \cos \gamma=R=\text { resistance, } \\
& Z \sin \gamma=X=\text { reactance. }
\end{aligned}
$$

Substituting these values in the last term, in (I), we have

$$
E^{2}=\varepsilon^{2}+Z^{2}\left(I_{d^{2}}+I_{w}{ }^{2}\right)+2 \varepsilon\left(R I_{w}+X I_{d}\right) .
$$

This equation enables the reactive current, $I_{d}$, to be calculated, as a function of the load, i.e., of the active current $I_{w}$; because, on solving with respect to $Z I_{d}$, we have

$$
Z^{2} I_{d}^{2}+2 \varepsilon \sin \gamma Z I_{d}+\varepsilon^{2}-E^{2}+Z^{2} I_{w}^{2}+2 \varepsilon Z I_{w} \cos \gamma=0 .
$$

Solving this quadratic equation for $I_{d}$, we have

$$
I_{d}=\frac{I}{Z}\left[-\varepsilon \sin \gamma+\sqrt{\varepsilon^{2} \sin ^{2} \gamma+E^{2}-Z^{2} I_{w^{2}}-2 \varepsilon Z I \cos \gamma}\right] .
$$

This expression will be put into another form later.

Application of the Diagram. Representation of Converter Operation with Constant Potential at Primary Terminals and at Brushes. If all that is desired is to maintain the potential constant at the terminals of the converter, the segment $O B=\varepsilon$ remains constant, and, in order to predetermine the different conditions of operation it is sufficient to let the point $C$, which defines the conditions of operation, describe a circle around $O$ as a center, with the constant radius $O C=E$. For each position of $C$ the load is measured by $d D$ and the

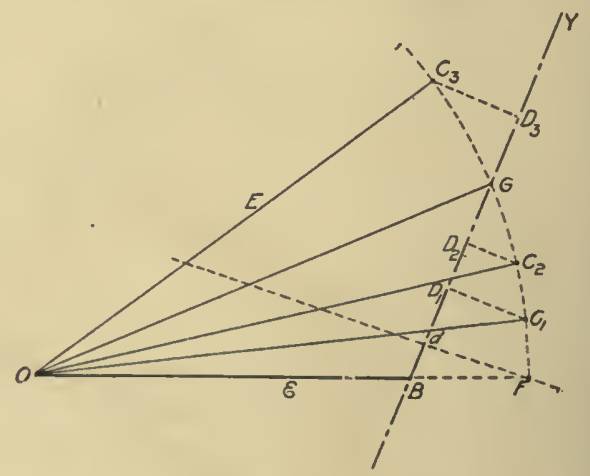

FIG. 4. reactive current by $C D$. It is thus seen, in Fig. 4 , that the reactive current, whicn is positive for light loads (since the characteristic point $C$ is to the right of the line $B Y$ ), diminishes as the load increases from the maximum value $F$ (secondary load alone) to zero, at $G$; and it then changes in sign, and begins again to increase. 
If the supply F.M.F., $E$, is varied, which amounts to the same thing as changing the radius $O C$ of the circle, the particular load for which the reactive current vanishes may be varied at will. For example, Fig. 5 (in which four circles of different radii are drawn), shows that it is possible to have the current in phase (power-factor equal to unity) with a certain useful load $d C_{2}$ or with zero load, instead of the load $d G$, and that it is possible even to have negative lag (or a leading current) at all loads, by suitable variation of the supply E.M.F. E.

It is seen that in any case it is impossible to prevent the reactive current from varying when the load changes. Since this current produces a magnetizing or a demagnetizing action equal to that of

$$
K \frac{N}{2} I_{d} \sqrt{2} \text { inductive ampere-turns, }
$$

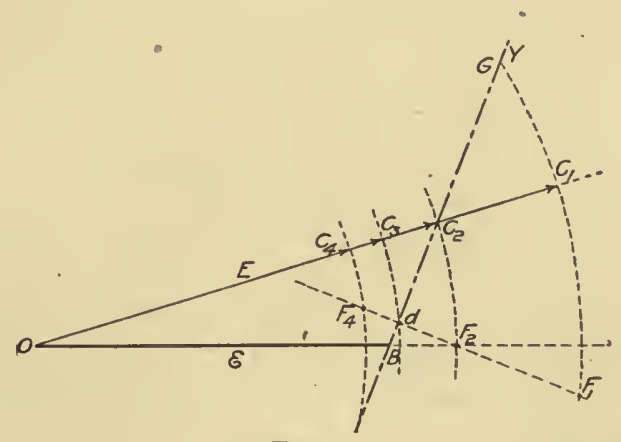

Fig. 5.

it is not possible to maintain constant potential by means of constant excitation if the impedance of the circuit is not negligible, but that it will be necessary to increase or decrease the excitation ampereturns by an amount equal and contrary to that of the ampere-turns of the reactive current in order that the total inducing flux may remain constant. (The variation of the magnetic leakage may introduce a slight complication, as will be seen later.)

With a given value of $\gamma$, if the impedance $Z$ is varied, we see that, the greater its value, the larger the scale of amperes will be, and, consequently, the smaller will be the variation of load which corresponds to a given angular displacement of the vector $O C$. The variations of the active current with the load will therefore increase in proportion with the impedance. 


\section{GENERAL DIAGRAMS FOR SYNCHRONOUS MOTORS}

If the impedance is varied by changing only the reactance, $X$, without changing the resistance, $R$, the result is less clearly seen, because the direction of the reference line $O Y$ then changes at the same time as the scale of amperes. This point will be discussed later under the general case.

General Case. Reactive Current Values for a Given Voltage Variation as a Function of the Load. In the most frequent case the voltage is raised slightly when the load increases, to compensate for the line-losses.

In Fig. 6, let $E=$ the E.M.F. corresponding to zero-load, and let $E^{\prime}, E^{\prime \prime}, E^{\prime \prime \prime}$, etc., represent the successive values which $E$ must have when the load corresponds to the active currents $I_{w}{ }^{\prime}, I_{w}{ }^{\prime \prime}, I_{w}{ }^{\prime \prime}$,

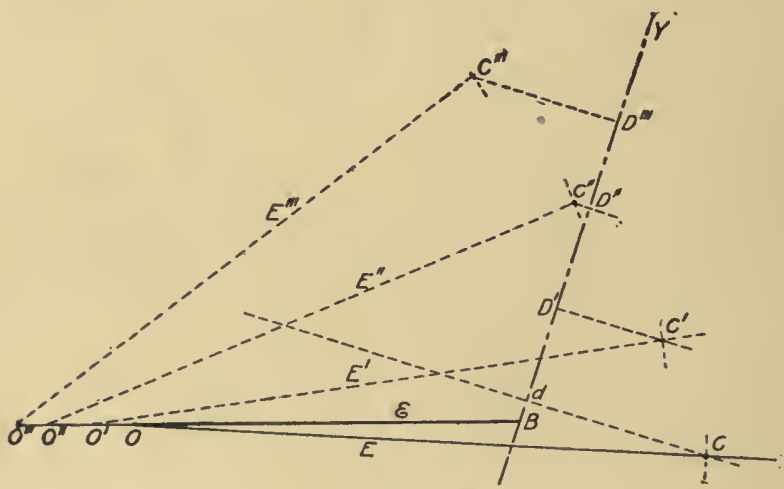

FIG. 6.

etc. These current-values are to be measured off on a reference-line $B Y$, starting from the point $B$, giving the distances $B D^{\prime}, B D^{\prime \prime}, B D^{\prime \prime \prime}$, etc., and the E.M.F.'s are to be measured off, along the horizontal line $B O$ starting from $B$ toward the left, giving the distances $B O$, $B O^{\prime}, B O^{\prime \prime}, B O^{\prime \prime \prime}$, etc.

From each of the " $O$ " points thus determined, as a center, let a circular arc be drawn, with the same radius, equal to the external E.M.F. E, which is a constant, assumed to be known. (In Fig. 6, in order to make the case mare general, it is assumed that the E.M.F.'s may be unequal.) The respective points of intersection of the circular arcs with the lines $d C, D^{\prime} C^{\prime}, D^{\prime \prime} C^{\prime \prime}, D^{\prime \prime \prime} C^{\prime \prime \prime}$, drawn perpendicular to the reference-line $B Y$, give the conditions corresponding to the various loads. 
The reactive currents corresponding to these loads are therefore entirely determined.

It will be seen that there is an infinite number of possible solutions, according to the value taken for the constant E.M.F. E. This E.M.F. can be made high or low, as desired: It depends on the ratio of transformation of the static transformers used for supplying current to the converters.

Moreover, the impedance $Z$ can also be regulated, within certain limits, at least, by the introduction of reactance in the current supplycircuit. (It is obviously desirable to reduce the ohmic resistance to the lowest possible value, to avoid lowering the efficiency.) It remains to be seen what are the most suitable values for $E$ and $X$. This point will be discussed in the next two sections.

Most Suitable Value of Current-Supply Voltage. This value is evidently that which gives zero-lag (unity-power-factor) for the most usual or frequent load, because we then have, for that load, a minimum current-value, consequently better line-efficiency, and better " over-all" efficiency. This condition of operation can be determined from the known or expected conditions of service, for the particular apparatus.

One might be tempted, on general principles, to take the maximum load as the one for which the supply-voltage is to be specially adjusted, so as to give the best results with that load, because it is precisely then that it is most important to reduce the losses; but since, in practice, it is necessary to consider the mean daily heating and efficiency of the apparatus used, it is for the average or normal load, and not for excessive loads of momentary duration only, that the supplyvoltage should be adjusted, with the object of obtaining unity powerfactor.

The range of voltage-regulation desired in the rotary converter can be obtained with more ease and precision when the point of maximum power-factor (" no lag ") corresponds to the average load. In fact, as will be seen later, the only converter-voltages (D.C.) which can be obtained exactly, by practical methods of excitation, are those corresponding to zero-lag and zero-load.

Most Suitable Value of Reactance. It is important to note, at the outset, that a certain minimum reactance is necessary in order to obtain, with a constant (A.C.) E.M.F. E, a (D.C.) voltage which will increase, or even remain constant, with the load.

Let us suppose that there is no reactance in the circuit. This makes $\gamma=0$; the reference-line $B Y$ (Fig. 7), will then coincide with 


\section{GENERAL DIAGRAMS FOR SYNCHRONOUS MOTORS}

the axis $O X$; and the currents corresponding to increasing loads will be represented by the distances $B d, B D^{\prime}, B D^{\prime \prime}$, etc. Let $D^{\prime \prime}$, for example, represent the normal load condition for which there is no lag $(\gamma=0)$. The vectors $C d, C^{\prime} D^{\prime}, C^{\prime \prime} D^{\prime \prime}$, which measure the corresponding reactive currents, increase extremely fast at light loads; and, since the scale of current-values is very small, owing to the fact that $Z$ is very small, it is seen that the reactive currents at light loads would be very large. On the other hand, in the case represented by Fig. 6, owing to the fact that the load-lines are no longer perpendicular to $O B$, it is easy to find, with a constant E.M..F. $E$, and with small active currents, load-points, $C, C^{\prime \prime}, C^{\prime \prime}$, corresponding to points

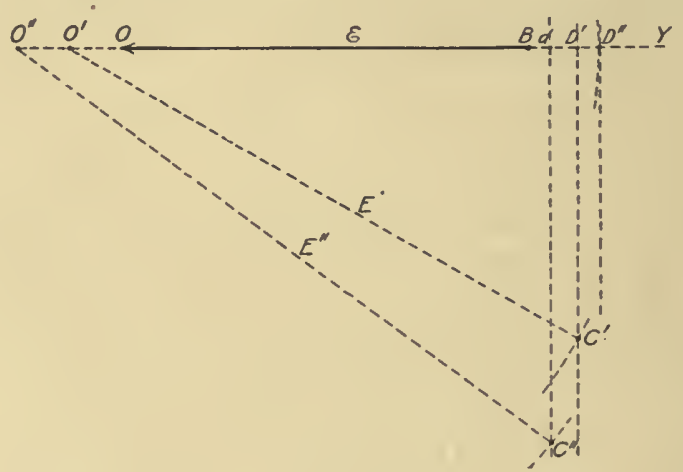

FIG. 7 .

$O, O^{\prime}, O^{\prime \prime}$, which are displaced to the left, and, consequently cause an increase in the voltage $\left(\frac{\varepsilon}{\xi}\right)$ at the brushes.

It is seen, thus, that it is possible to obtain economically a voltageregulation giving constant or rising voltage, as the load increases, only when the circuit contains a certain amount of reactance.

This point being established, it is easy to note the influence of reactance, by supposing that the "no lag" condition is made to correspond always to the same load, i.e., to the same value of the active current, $I_{w}$. In fact (Fig. 8), all the load-points corresponding to that condition are found to be located at distances, $Z I_{w}$, from the point $B$, which are proportional to the impedances $Z=\sqrt{R^{2}-X^{2}}$; hence, if we measure off, on $O B$ prolonged, a distance $B K$, equal to $R I_{w}$, all the points $G$, corresponding to different reactances $X$, will be located on the right line $K P$ perpendicular to $O K$. Likewise, 
the point of zero-load, $d$, will be on the perpendicular $k d$ corresponding to a segment $B k=R j_{0}$. We will compare, for various cases, the values of the reactive current $i_{0}$ corresponding to this no-load condition.

This reactive current is obtained by drawing through $d$ the right line $d F$, perpendicular to $B G$, until it crosses the circle of radius $E$. In order to make this current-value comparable with $B K$, it is only necessary to reduce also the segment $d F$ in the proportion $\frac{R}{Z}$, which can be done by projecting it on $K G$. The segment $d^{\prime} F^{\prime}$ then measures the value $R i_{0}$, while $B k$ measures $R I_{w}$. By this process all the current values are referred to one

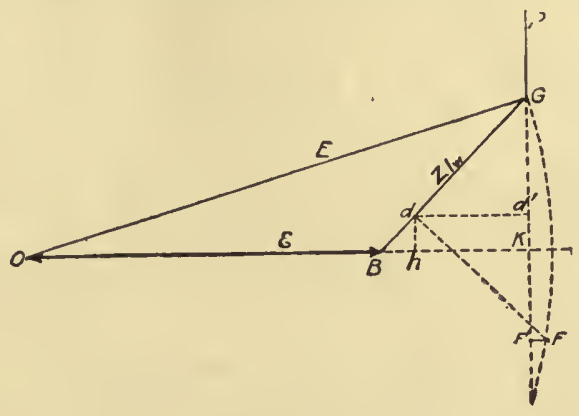

Fig. 8. and the same scale, $i_{0}$, whatever may be the value of $Z$. On making the diagram anew for cases corresponding to various angles of the line of reference $B G$, it is found that the reactive current, which is very

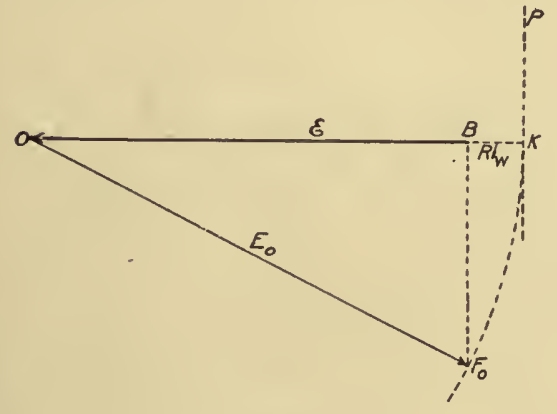

FIG. 9. large for very small "lags" diminishes, rapidly at first, then more slowly.

In order to follow more easily the law of variation, let us suppose the active current to vanish at zeroload, i.e., let us assume $j_{0}=0$, which makes $d$ coincident with $D$ (Fig. Io). The reactive current $i_{0}$ is then proportional simply to the distance $F H$.

If the point $G$ be raised gradually on the vertical line $K G$, starting from the point $K$, the reactive current is at first equal to $\frac{B F_{0}}{R}$ (Fig. 9), and it will then decrease; for example, for the point $G$ (Fig. Io), it will decrease to $F H$. When $G$ is very high, it is seen that an additional rise, $G^{\prime} G^{\prime \prime}$, for example, will increase almost proportionally the 
radius $E$ without altering materially the inclination of the segment $B F^{\prime}$. Hence, if the reactance is increased beyond a certain point the reactive current $i_{0}$, corresponding to zero-load, will increase with it.

This reactive current must therefore have a minimum; and to every value higher than this minimum there will correspond two

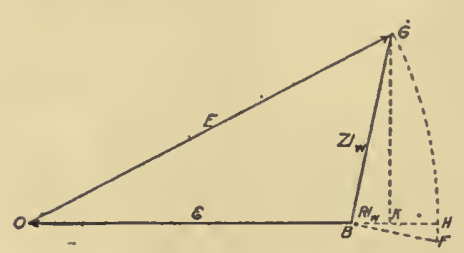

Fig. 10. reactance values, and two inclinations of the line $B G$, one greater, the other smaller.

We can learn more about this minimum by calculations based on the fact that the same value of the supply-voltage $E$ enables both the "no-lag" point $G$ and the "no-load" point $F$ to be obtained.

To make the case more general, let us suppose a condition wherein $j_{0}$ does not vanish and wherein the voltage with load, $\varepsilon^{\prime}$, is different from the voltage $\varepsilon$ without load. We will have the two following relations:

At the point $G, \quad E^{2}=\varepsilon^{\prime 2}+Z^{2} I_{w}{ }^{2}+2 \varepsilon^{\prime} R I_{w}$. . . . (a)

At the point $F, \quad E^{2}=\varepsilon^{2}+Z^{2} i_{0}^{2}+2 \varepsilon X i_{0}$.

From these, eliminating $E$, we have

$$
\varepsilon^{\prime 2}+Z^{2} I_{w}^{2}+2 \varepsilon^{\prime} R I_{w}-\varepsilon^{2}-Z^{2} i_{0}-2 \varepsilon X i_{0}=0 .
$$

This is the condition required. Solving for $i_{0}$ and then for $X$, we obtain the two following formulas:

$$
\begin{aligned}
& i_{0}=\frac{-\varepsilon X+\sqrt{\varepsilon^{2} X^{2}+Z^{2}\left(\varepsilon^{\prime 2}-\varepsilon^{2}+Z^{2} I^{2}{ }_{w}+2 \varepsilon^{\prime} R I_{w}\right)}}{Z^{2}} ; \ldots . \\
& X=\frac{\varepsilon i_{0} \pm \sqrt{\varepsilon^{2} i_{0}^{2}-\left(I_{w}{ }^{2}-i_{0}^{2}\right)\left[\varepsilon^{\prime 2}-\varepsilon^{2}+R^{2}\left(I_{w}{ }^{2}-i_{0}^{2}\right)+2 \varepsilon^{\prime} R I_{w}\right]}}{I_{w}{ }^{2}-i_{0}^{2}} .
\end{aligned}
$$

In (5) the expression under the radical sign is taken with the + sign only, because, for each value of $X, i_{0}$ can have only one value, which, necessarily, is positive. On the other hand it has been seen that $X$ has two values for each value of $i_{0}$; hence it is necessary to retain both signs $( \pm)$ before the expression under the radical sign, in Eq. (6). 
The minimum value of $i_{0}$ could be obtained by making the derivative of Eq. (5) equal to zero and solving; but it is simpler to equate to zero the expression under the radical sign in Eq. (6), because the minimum value of $i_{0}$ is evidently that below which the value of $X$ would become imaginary. Hence, the positive and negative quantities under the radical sign must offset each other, when there is a minimum.

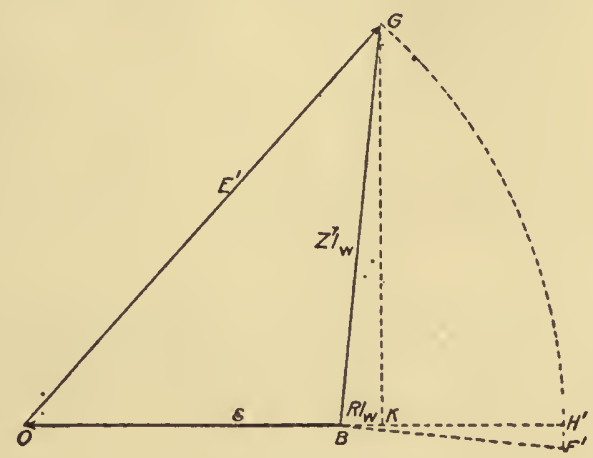

FIG. II.

Equating the quantity under the radical sign in (6) to zero, and simplifying with respect to $\left(I_{w}^{2}-i_{0}^{2}\right)$, instead of $i_{0}^{2}$, we have

$$
\varepsilon^{2} I_{w}^{2}-\left(I_{w}^{2}-i_{0}^{2}\right)\left[\varepsilon^{\prime 2}+2 \varepsilon^{\prime} R I_{w}-R^{2}\left(I_{w}^{2}-i_{0}^{2}\right)^{2}\right]=0 . \quad . \quad .
$$

Since the ohmic drop of voltage should be only a small fraction of $E$, the last term, $R^{2}\left(I_{w}{ }^{2} i_{0}\right)$ may be neglected, and we thus obtain the approximate value

$$
\left(I_{w}^{2}-i_{0}^{2}\right)=\frac{I_{w} E_{1}^{2}}{\varepsilon^{\prime 2}-\varepsilon^{2}+2 \varepsilon^{\prime} R I_{w}},
$$

and the minimum value of $i_{0}^{2}$ is

$$
i_{0}^{2} \text { minimum }=\frac{\varepsilon^{\prime 2}-\varepsilon^{2}+2 \varepsilon^{\prime} R I_{w}}{\varepsilon^{\prime 2}+2 \varepsilon^{\prime} R I_{w}} I_{w}^{2} .
$$

Since the quantity under the radical sign in (6) practically vanishes for this minimum value of $i_{0}^{2}$, the expression for $X$ which corresponds to the minimum reactive current $i_{0}$ is, simply, by approximation,

$$
X_{0}=\frac{\varepsilon i_{0}}{I_{w}^{2}-i_{0}^{2}}
$$


or, substituting for $i_{0}$,

$$
X_{0}=\frac{\left(\overline{\left(\varepsilon^{\prime 2}+2 \varepsilon^{\prime} R I_{w}\right)\left(\varepsilon^{\prime 2}+2 \varepsilon^{\prime} R I_{w}-\varepsilon^{2}\right)}\right.}{\varepsilon I_{w}} .
$$

This is the most suitable reactance-value for reducing $i_{0}$ to a minimum. Inasmuch as, on the other hand, the supply-voltage required increases with the reactance $X$, and inasmuch as this voltage must be kept as low as possible in order to utilize the apparatus to the greatest advantage (i.e., to obviate the necessity of making it too large and, consequently, more expensive), for the effective output, we may conclude that, in general, the reactance value should be comprised between zero and the value $\left(X_{0}\right)$ corresponding to the minimum zero-load reactive current.

Note 1.-Formulas $5,6,7,8$ can be put into a form which is often more convenient practically, by expressing the values of $i_{0}$ in terms of $I_{w}$, the values of $R I$ in terms of $\varepsilon$, the values of $\varepsilon^{\prime}$ in terms of $\varepsilon$, and the values of $X$ in terms of $R$.

Let us take

$$
\frac{\varepsilon^{\prime}}{\varepsilon}=(\mathrm{I}+\varepsilon) ; \quad \frac{R I_{w}}{\varepsilon}=\alpha ; \quad \frac{X}{R}=m ; \quad \frac{i_{0}}{I_{w}}=\tan \phi ;
$$

$\cos \phi$ being the power-factor, and $m$ being the value of $\tan \gamma$.

From these equations, we deduce

$$
\begin{aligned}
& \frac{X I_{w}}{\hat{\varepsilon}}=\alpha m, \\
& \frac{\varepsilon}{Z}=\frac{I_{w}}{\alpha \sqrt{I+m^{2}}},
\end{aligned}
$$

and also the equivalent formulas:

$$
\begin{gathered}
i_{0}=\frac{I_{w}}{\alpha\left(1+m^{2}\right)}\left\{-m+\sqrt{m^{2}+\alpha^{2}\left(\mathrm{I}+m^{2}\right)^{2}+\left(\mathrm{I}+m^{2}\right)\left(\varepsilon^{2}+2 \varepsilon+2 \alpha(\mathrm{I}+\varepsilon)\right\}} \cdot\left(5^{\prime}\right)\right. \\
X=\frac{R \tan \phi}{\alpha\left(\mathrm{I}-\tan ^{2} \phi\right)} \pm R \sqrt{\frac{\tan ^{2} \phi}{\alpha^{2}\left(\mathrm{I}-\tan ^{2} \phi\right)}-\frac{\frac{2}{\alpha}(i+\varepsilon)+\frac{s^{2}+2 \varepsilon}{\alpha^{2}}}{\mathrm{I}-\tan ^{2} \phi}-\mathrm{I}, \quad\left(6^{\prime}\right)}
\end{gathered}
$$

minimum of

$$
i_{0}{ }^{2}=I_{w}{ }^{2} \frac{(\mathrm{I}+\varepsilon)^{2}-\mathrm{I}+2 \alpha(\mathrm{I}+\varepsilon)}{(\mathrm{I}+\varepsilon)^{2}+2 \alpha(\mathrm{I}+\varepsilon)}, .
$$

$$
X_{0}=\frac{R}{\alpha} \sqrt{\left[(\mathrm{I}+\varepsilon)^{2}+2 \alpha(\mathrm{I}+\varepsilon)\right]\left[\varepsilon^{2}+2 \varepsilon+2 \alpha(\mathrm{I}+\varepsilon)\right]} .
$$


In the particular case where the voltage must be constant, we must have $\varepsilon^{\prime}=\varepsilon$, or $\varepsilon=0$, and the expression for $i_{0}^{2}$ reduces to the simple form

$$
i_{0}^{2}=I_{w}^{2} \frac{2 \alpha}{1+2 \alpha}
$$

which gives, substantially,

$$
i_{0}=I_{w} \sqrt{2 \alpha} \quad \text { and } \quad X_{0}=\frac{R}{\alpha} \sqrt{2 \alpha(\mathrm{I}+2 \alpha)}
$$

or, practically,

$$
X_{0}=R \sqrt{\frac{2}{\alpha}} .
$$

NoTE 2.- In the particular case where $i_{0}=I_{w}$ and $\tan \phi=\mathbf{I}$, formulas $(7)$ and $\left(7^{\prime}\right)$ will no longer apply, and they must be replaced by the following formulas, deduced from Eq. (4):

$$
\begin{aligned}
& \left.X=\frac{\varepsilon^{\prime}}{\varepsilon} \frac{R I_{w}}{i_{0}}-\frac{\varepsilon^{\prime 2}-\varepsilon^{2}}{2 s i_{0}}, \ldots . . . \quad . \quad . \quad .7 a\right) \\
& X=\frac{R}{\tan \phi}\left[(I+\varepsilon)-\frac{\varepsilon^{2}+2 \varepsilon}{2 \alpha}\right] \ldots . . . \quad . \quad\left(7 a^{\prime}\right)
\end{aligned}
$$

\section{Regulation of Voltage at Terminals by Variation of the Supply} E.M.F. In what precedes it has been supposed that the E.M.F. E of the source of current is constant at all loads. This necessitates a reactive current at light loads.

It is possible to compound or to regulate automatically the excitation of the alternator in such manner that its E.M.F., F, will increase with the load. This compounding will obviate all necessity for any reactance; and the line of reference in the diagram can be the line $O B$ prolonged, if there is no other loss of voltage than that due to ohmic drop. This solution is obviously the most perfect one, theoretically, but it cannot be realized exactly otherwise than in the exceptional case where the rotary converters are supplied individually by separate alternators. It is not possible otherwise to establish a correlation between the load of the converters and that of the lines when they supply current at the same time to other apparatus.

An approximation to this method of regulation can be made by supplying the converters with current through transformers whose 


\section{0}

ratio of transformation is made variable by means of "taps" on the the secondary winding connected with suitable switching apparatus.

The fundamental diagram (Fig. I2), or the formula (a), will determine the values which should be given to $E$ for each value of the E.M.F. $\varepsilon$, and of the load $Z I_{w}$, measured off along the line $B Y$ corresponding to the fixed reactance of the circuit. The most suitable value for the reactance is, in this case, evidently, the lowest value, $X=0$, when possible; because this value reduces to a minimum the values of the E.M.F. $E$ required. Therefore, no supplementary reactance should be included in the circuit. Under these circumstances $B Y$ becomes the prolongation of $O B$ in the diagram.

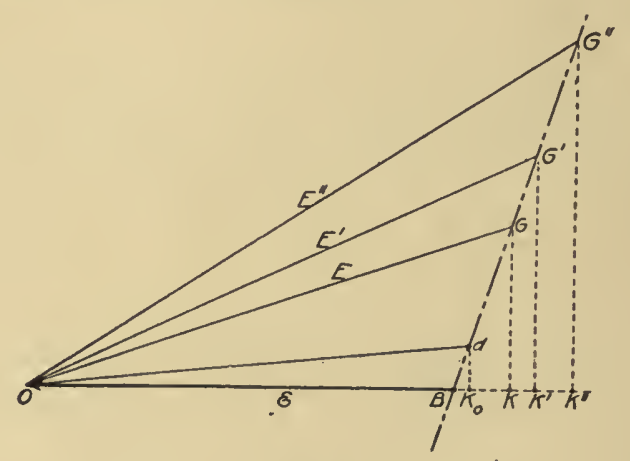

FIG. I2.

Regulation of Voltage at Terminals by Variation of Reactance. It is also possible to neutralize the lag at all loads more simply by keeping the supply E.M.F. $E$ constant (and equal to that required to insure the necessary voltage at the terminals with full load without reactive current), and then reduce this voltage at lighter loads by the introduction of suitable reactances in the circuit.

Fig. I 3 explains this method of regulation in the case where the E.M.F. $E$ is constant; and it enables the characteristic features of this case to be studied.

Let $B K=R I_{w}$, represent the loss of voltage by ohmic drop in the circuit, with full load. The segment $B K$ can serve to represent the acti e current.

Let $B K,{ }^{\prime} B K$, , etc., represent intermediate current values; and let the corresponding perpendiculars $K G, K^{\prime} G^{\prime} K^{\prime \prime} G^{\prime \prime}$ be drawn. Their points of intersection $G, G{ }^{\prime} G^{\prime \prime}$, with the circle of radius $E$, 
described around the center $O$, will define the load-conditions corresponding to these currents.

In order to obviate reactive currents it is sufficient, for each case, that the reactance should be adjusted to a value exactly equal to that

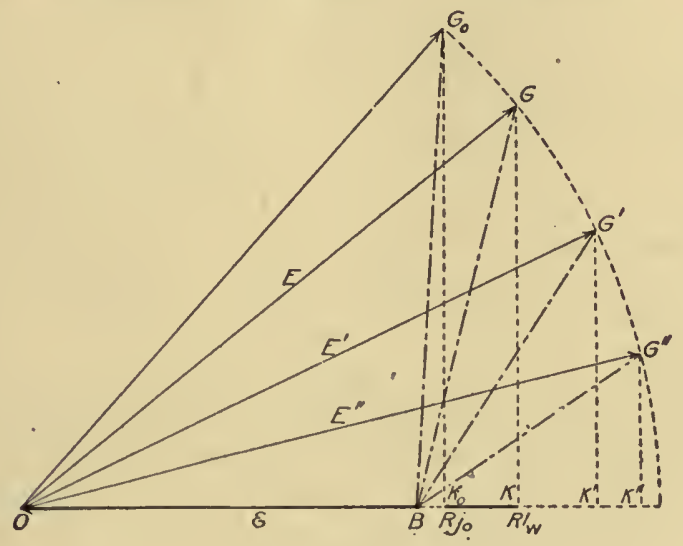

FIG. I3.

which makes the reference line pass through $G$. This means that the reactance $X$ must be made successively equal to the following values:

$$
\frac{G K}{B K} R ; \quad \frac{G^{\prime} K^{\prime}}{B K^{\prime}} R ; \frac{G^{\prime \prime} K^{\prime \prime}}{B K^{\prime \prime}} R ; \text { etc. }
$$

These values of $X$ can be calculated for each value of $I_{w}$, by the Eq. (a) given herein above. From this equation

$$
E^{2}=\varepsilon^{\prime 2}+Z^{2} I^{2} w+2 \varepsilon^{\prime} R I_{w}, \quad . \quad . \quad . \quad . \quad .
$$

on substituting for $Z^{2}$ its value $\left(Z^{2}=R^{2}+X^{2}\right)$, and solving for $X^{2}$, we have

$$
X^{2}=-R^{2}+\frac{E^{2}-\varepsilon^{\prime 2}-2 \varepsilon^{\prime} R I_{w}}{I_{w}^{2}} .
$$

Practically, this regulation can be worked by hand or even automatically, by making use of reactance-coils having movable cores, which are pushed all the way in when the load is zero, and are gradually 
pulled out as the load increases, in such a way as to bring back to zero a direct-reading phase-indicator, connected at the terminals of the converters.

This method is applicable whatever may be the value of $E$ (i.e., the radius of the circle); but the most suitable value is, obviously, the smallest value, which corresponds to $E=\varepsilon^{\prime}+R I$.

The diagram presupposes that the locus of the point $G$ is a circle; but if the E.M.F. $\varepsilon$ varies the center $O$ of that circle is displaced and the locus of $G$ is a curve, which can be drawn by points determined from the data. This does not, in any way, necessitate modifications in the method.

Power-Factor of the Generator. In what precedes, we have seen the necessity of keeping dorm the value of the E.M.F., $s$. It is customary to express the disadvantages of the useless increase of this E.M.F. by stating the value of the power-factor, i.e., by the value of the cosine of the phase-angle, usually termed the "angle of lag," between the E.M.F. and the current.

Let $\psi$ denote this phase-angle, and let $\theta$ denote the phase-angle between $\varepsilon$ and $E$; and let, as before, $\phi$ denote the phase-angle between the current and the E.M.F. $\varepsilon$. We have

$$
\tan \phi=\frac{I_{d}}{I_{w}}
$$

and this angle of lag, $\phi$, is always in the same direction as 0 (see Fig. r), i.e., in the direction of the hands of a watch (in these diagrams). We therefore have

$$
\phi=\theta+\phi
$$

For the condition of average load, that is to say, in the cases where there is no lag, we have $\phi=0$, and, therefore, $\psi$ reduces to the same value as $\theta$ itself.

In order to increase the power-factor of the generator as much as possible at average loads, we should, therefore, reduce $\theta$, and, consequently, $\varepsilon$, as much as possible, as already stated. It is much more important to do this than to reduce to zero the " no-load " lag between the current and the converter E.M.F. Therefore, the reactance used should have a value comprised between zero and the value which renders the no-load lag a minimum. This is all the more desirable 
because the lag $\theta$ then becomes negative at light loads, as indicated in Fig. If, and the value of $\psi$ is then a difference $\psi=\phi-\theta^{\prime}$, which increases but slowly with $\phi$.

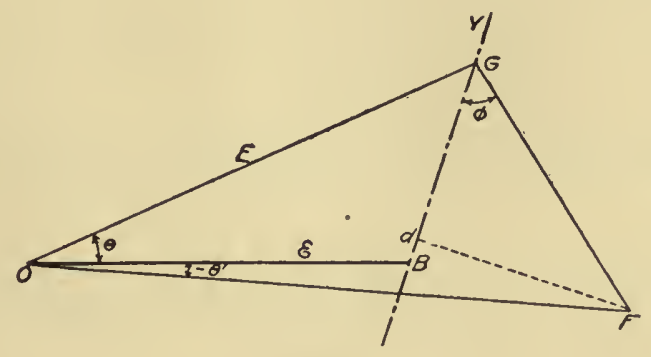

FIG. 14.

It is logical to endeavor to make $\theta$ and $\phi-\theta^{\prime}$ equal to each other, in such a way as to render the two extreme values of $\psi$ as egual and as small as possible. 


\section{CHAPTER II}

\section{PREDETERMINATION OF THE FIELD-EXCITATION OF ROTARY CONVERTERS}

IN the preceding chapter we discussed the conditions attending the supply of electric current to rotary converters, and, in particular, the effects of reactance in the supply-circuit, and the various ways of using it to obtain a given range of impressed voltage for a given range of load without exceeding a certain maximum reactive current, and even while making this current approach a minimum value. There still remains the second portion of the problem to be solved, namely, the determination of the field-exciting ampere-turns, due to either the series or the shunt-winding coils, which enable the rotary converter to follow approximately this law of variation, in consequence of the effects of the reactive currents.

In what follows it will be assumed that we are dealing with the ordinary case of a converter which is operating without lag, at its average load, and which consequently, has, at lighter loads, a positive reactive current (lagging $\frac{\pi}{2}$ behind the E.M.F.) whereby there is produced a magnetizing effect on the field. ${ }^{1}$ This is the case which was discussed in the preceding chapter.

Characteristic Features of the Rotary Converter. We will begin by considering the magnetic features of the rotary converter, which involve two new important elements:

1. The excitation-curve, or the curve showing the variation of induced E.M.F. as a function of the field-exciting ampere-turns due to the field-windings. This curve is supposed to be known from the shop-tests made of the machine at the time it was finished.

In the excitation-curve (Fig. I5) the ordinates represent the E.M.F.'s

${ }^{1}$ It is easy to see that any reactive current which lags behind the impressed E.M.F. $E_{1}$ is leading with respect to the internal E M.F. $\varepsilon$, since $E$ and $\varepsilon$ are practically in opposition. 
(volts) induced, and the abscissas represent the corresponding ampereturns of excitation.

2. The equivalent magneto-motive force of the armature, i.e., the number of ampere-turns which, if acting upon the field-cores (same as the ampere-turns of the regular field-winding) would produce in the magnetic circuit the same magnetomotive force as is produced by the reactive current $I_{d}$ circulating through the armature.

'These equivalent average ampere-turns can be represented, as in an alternator, by the expression,

$$
K \frac{N^{\prime}}{2} I_{d} \sqrt{2}
$$

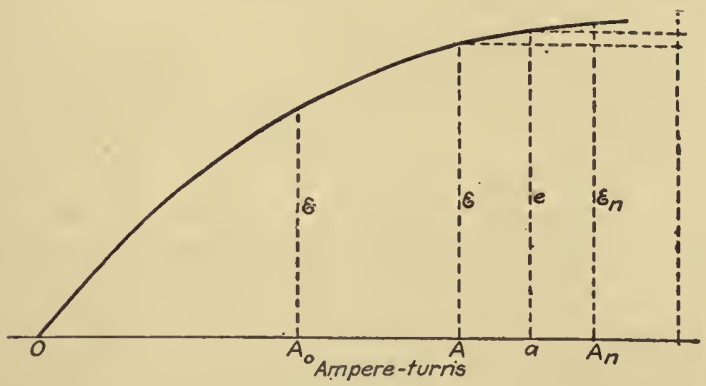

FrG. 15 .

in which $N^{\prime}=$ the number of peripheral wires of the armature-winding per double field (i.e., per magnetic circuit); $K$ being a coefficient of reduction which depends upon the number of phases and of armature slots, and on the width of the poles; and $I_{d}$ being the effective value of the reactive current.

$K$ is determined by calculation, by taking $I_{d}=\mathrm{I}$ ampere, with zero-lag, and calculating the mean magnetic potential produced under one pole.

The value of $K$ can be obtained by experimental measurement. This can be done with an approximation which is generally sufficient for all purposes (since the leakage-reactance, ws, is practically negligible), by running the converter at normal speed by power applied mechanically, and then making it supply (on the A.-C. side) with normal voltage, a purely inductive external circuit (composed of a reactance giving a power-factor-cos $\phi$-equal, at most, to 0.20 ); the difference between 


\section{$196^{\circ}$ GENERAL DIAGRAMS FOR SYNCHRONOUS MOTORS}

the voltage $(\varepsilon)$ at the terminals, with this load, and the voltage (e) obtained with open circuit, with the same excitation, is the measure, in volts, of the armature-reaction corresponding to the current supplied to the external circuit by the converter.

Taking the values $\varepsilon$ and $e$ thus obtained and referring them to the excitation-curve (Fig. I5), they will correspond to different points on this curve. The difference between the abscissas, $A$ and $a$, corresponding to these two points will be a measure of the equivalent ampereturns of the armature (neglecting, as before, the effects due to magnetic leakage). It is only necessary, in order to obtain $K$, to divide $a-A$ by the product of the effective reactive current $I_{d}$ measured in the abovementioned experimental test, by half the number of turns of wire, $\frac{N^{\prime}}{2}$, and by the constant $\sqrt{2}$. We will have

$$
K=\frac{a-A}{\frac{N^{\prime}}{2} I_{d} \sqrt{2}^{-}}
$$

Compound-Excitation. Different Factors of this Excitation. Rotary converters are excited by current taken from their secondary (D.-C.) side. The field-excitation may, obviously, be either shunt, series, or compound.

Let $E_{2}=$ secondary voltage at the terminals;

$I_{2}=$ secondary or output current delivered by the rotary converter;

$n=$ number of turns of the series-winding;

$r=$ resistance of one turn of the shunt-winding.

The shunt ampere-turns and the series ampere-turns will be, respectively, equal to

$$
\frac{E_{2}}{I} \quad \text { and } \quad n I_{2} \text {. }
$$

It is these ampere turns which, conjointly with the equivalent ampere-turns of the armature, determine the total excitation.

In the most general case, that of a compound-wound converter, the question is, therefore, quite complex, since there are three variable factors, the first of which varies with the voltage, the second with the output (direct) current, and the third with the reactive (alternating) current.

As already seen, the secondary ampere-turns are proportional to the ampere-turns due to the useful primary current, $I_{w}-j_{0}$. 
Let $\xi$ represent the ratio of transformation, i.e., the ratio of the primary E.M.F. to the secondary E.M.F. Since the armature-resistance is already, by hypothesis, counted once in that of the supply-circuit, it need not be counted again here. We can, therefore, write:

Ampere-turns of shunt excitation,

$$
A_{d}=\left(\frac{\mathrm{r}}{\xi_{r}}\right) \varepsilon ; \quad . \quad . \quad . \quad . \quad . \quad . \quad .
$$

Ampere-turns of series-excitation, ${ }^{1}$

$$
A_{s}=n I_{2}=\left(\frac{\pi k n}{\sqrt{2}}\right)\left(I_{w}-j_{0}\right) ; \quad . \quad . \quad .
$$

Ampere-turns of reactive currents, produced by the armature,

$$
A \alpha=\left(\frac{K N^{\prime}}{\sqrt{2}}\right) I_{d}
$$

The magnetomotive forces are thus expressed directly in terms of the elements of the primary currents. This will enable the excitations for each load-condition to be calculated.

If we count as positive the lagging reactive currents (which are magnetizing currents) the total number, $A$, of exciting ampere-turns corresponding to the load-condition $\varepsilon I_{w}$ will therefore be

$$
A=\frac{\varepsilon}{\xi r}+\frac{\pi k n}{\sqrt{2}}\left(I_{w}-j_{0}\right)+\frac{K N^{\prime}}{\sqrt{2}} I_{d}
$$

The + sign is placed before the reactive ampere-turns because, under the normal conditions of current-supply previously considered, the reactive current is directed to the right of $D$, i.e., it is positive and magnetizing between zero-load and normal load, and it is demagnetizing beyond normal load. The + sign is placed before the series ampere-

${ }^{1}$ From the relation found at the beginning of Chapter I, namely, $\frac{I_{2}}{I_{w}}=\frac{\pi k}{\sqrt{2}}$, we get $I_{2}=\frac{\pi K}{\sqrt{2}} I_{w}$. In Eq. (I8), for the sake of greater precision, $I_{w}$ is replaced by $I_{w}-j_{v}$. If the armature had two different windings, the ratio would have to be multiplied by that of the numbers of turns of these two windings, $\frac{N}{N_{2}}$. 


\section{GENERAL DIAGRAMS FOR SYNCHRONOUS MOTORS}

turns because, in general, the series-winding must work concordantly (or cumulatively) with the shunt-winding, as will be seen later.

The unknown constants, $r$ and $n$, of the windings, are easily determined when the E.M.F., $\varepsilon_{0}$ for zero-load, and the E.M.F. $\varepsilon_{m}$ for the mean load (corresponding to the current $I_{m}$, with zero-lag) are known.

The excitation-curve (Fig. I5) gives the ampere-turns, $A_{0}, A_{n}$, corresponding, respectively, to the E.M.F.'s $\varepsilon_{0}$ and $\varepsilon_{m}$. For the normal load-condition we will have

$$
A_{m}=\frac{\mathrm{I}}{\xi r} \hat{\varepsilon}_{m}+\frac{\pi k n}{\sqrt{2}}\left(I_{m}-j_{0}\right) .
$$

For the no-load condition we will have

$$
A_{0}=\frac{\mathrm{I}}{\xi r} \varepsilon_{0}+\frac{K N^{\prime}}{1_{2}} i_{0}
$$

As already seen, the value of the reactive current corresponding to zero-load cannot fall below a certain minimum. If a minimum value be assumed for this current, Eq. (22) can be used to determine the conductance of one turn of the shunt winding. From Eq. (22), solving for $\frac{\mathrm{I}}{r}$, we have

$$
\frac{\mathrm{I}}{r}=\frac{\xi}{\varepsilon_{0}}\left(A_{0}-\frac{K N^{\prime} i_{0}}{\sqrt{2}}\right) .
$$

From Eq. (2I), solving for $n$, and substituting for $\frac{\mathrm{I}}{r}$ the value given in Eq. (23), we have

$$
n=\frac{A_{m}-\frac{\varepsilon_{m}}{\xi r}}{\pi k\left(I_{m}-j_{0}\right)} \sqrt{2}=\frac{\left(A_{m}-\frac{\varepsilon_{m}}{\varepsilon_{0}} A_{0}\right) \sqrt{2}+\frac{\varepsilon_{m}}{\varepsilon_{0}} K N^{\prime} i_{0}}{\pi k\left(I_{m}-j_{0}\right)} .
$$

It is seen that the shunt ampere-turns, which are proportional to $\frac{\varepsilon_{m}}{r}$, are less than the total ampere-turns $A_{0}$ (on the assumption that $\left.\varepsilon_{m}>\varepsilon_{0}\right)$, and that they decrease when the reactive current $i_{0}$ increases; whereas the series ampere-turns, which are proportional to $n\left(I_{m_{\iota}}-j_{0}\right)$ increase with the reactive current $i_{0}$.

Determination of Reactive Current as a Function of the Excitation, when the Active Current is Constant, then when the Power 
is Constant, the Generator E.M.F. being always Constant. Let us suppose, as in the case of synchronous motors, that the converter is supplied by a constant E.M.F. E, and excited by an exciting current which can be regulated at will.

The fundamental diagram (Fig. 2), combined with the preceding curve (Fig. I6), will enable us to find readily, for each value of the active current, $I_{w}$, the values of reactive current corresponding to each value of the excitation, expressed in ampere-turns.

Let, for example, $I_{10}$ be the given active current, and $B D$ the line of reference, which is drawn after the value of $\gamma$ has been obtained. The locus of the point $C$, characteristic of the load-conditions, for that constant value of $I_{w}$, is the right line $D Q$, drawn perpendicular to $B D$, at the distance $B D=Z I_{w}$, whereas the locus of the point $O$ the second end of the vector $E$, is the line $O B X$.

All the load-conditions corresponding to the active current $I_{w}$ will therefore be obtained by drawing, from the first points $C, C^{\prime \prime}, C^{\prime \prime \prime}, \ldots$, on the first right line, circular arcs of radius $E$, intersecting the second line at the points

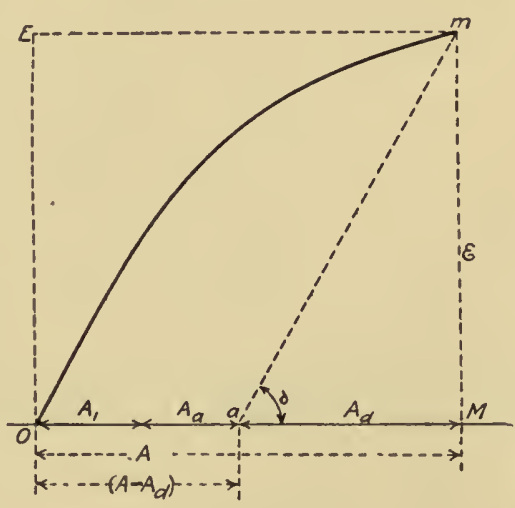

Fig. I6. $O, O^{\prime}, O^{\prime \prime}, \ldots$ The segments $B O, B O^{\prime}, B O^{\prime \prime}, \ldots$ thus formed measure the values of the corresponding internal E.M.F.'s, $\varepsilon, \varepsilon^{\prime}$, $\varepsilon^{\prime \prime}, \ldots$, and the segments $D C, D C^{\prime}, D C^{\prime \prime}, \ldots$ divided by $Z$, measure the values of the reactive current $I_{d}$.

For each pair of values of $\varepsilon$ and $I_{d}$, thus determined, the corresponding ampere-turns of excitation, $A_{e}$, are obtained, from the excitationcurve (Fig. 16), by subtracting the counter ampere-turns of the armature from the resultant ampere turns $(A)$ corresponding to the ordinate $(\varepsilon)$ of this curve; whence we have

$$
A_{\epsilon}=A \mp \frac{K N^{\prime}}{\sqrt{2}} I_{d}
$$

It should be remembered that the reactive current $I_{d}$ is demagnetizing and positive if it is counted to the right of the axis $B D$, and that 


\section{GENERAL DIAGRAMS FOR SYNCHRONOUS MOTORS}

it is magnetizing and negative if counted to the left. The upper sign applies in the first case, and the lower sign in the second case. Under those conditions the diagram can now be used for determining the locus of the points $C$ and the variations of $E$, when the power is constant, if the generator E.M.F. be maintained constant as in the case of synchronous motors. We have in this case, simply,

$$
P=\varepsilon I_{w},
$$

so that, for each value of $I_{v}$, and, consequently, for each position of the right line $D Q$, the value of $\varepsilon$, and, therefore, the position of the point $O$, are known; and, from these points, circles of radius $E$ can be drawn,

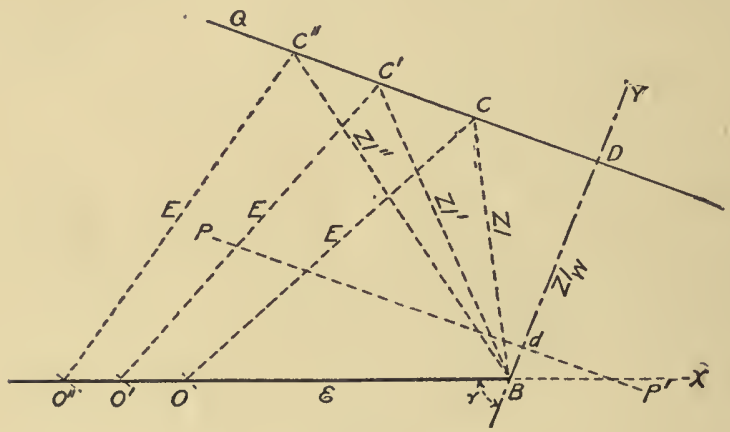

FIG. I7.

whose respective intersections with the right lines $D Q$ give the $C$ points required. It is possible, thus, to draw the " $\mathrm{V}$ " curves analogous to those of synchronous motors, and the curves of the corresponding . values of $\varepsilon$. But these load-conditions for constant power no longer present in the case of rotary converters the same practical interest as in the case of synchronous motors, because the variation of $\varepsilon$ would be very troublesome.

Different Values of the Excitation, with Constant Power and Constant Potential. V-Curves for Constant Potential. The preceding diagram (Figs. I6 and I7) also gives the immediate solution of the more practical problem ${ }^{1}$ which consists in determining the current-values, $I$, corresponding to different excitations, with constant

1 The case considered is, as yet, more of theoretical than of practical interest, because it assumes that the E.M.F. is maintained absolutely constant, a condition which, as will be seen later, can be realized only approximately. In the gencral case therefore both $\varepsilon$ and $E$ will vary. 
power, and with the secondary voltage constant at the brushes. It is the same problem as the preceding one except that it is not the difference of potential at the terminals, but the induced E.M.F. $\varepsilon$, which must be supposed constant, in this case.

The locus of the point $C$ is again the right line $C D$ itself. But, as we have added the condition that the segment $O B$ remains constant, the point $O$ will, therefore, remain fixed (Fig. I $7 a$ ).

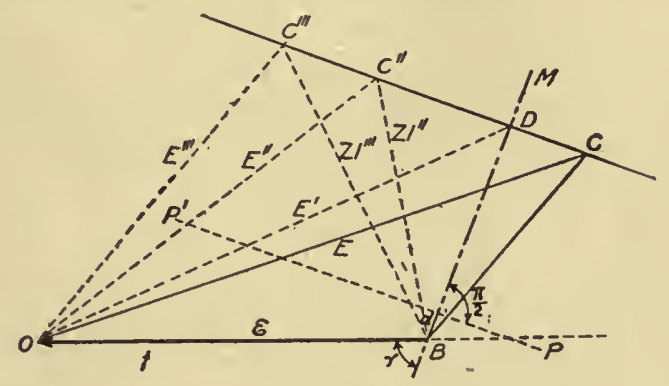

FIG. I7a.

For each position of the point $C$ the corresponding segment $C B$ indicates the current, and $O C$ indicates the E.M.F. required at the generator. Taking the E.M.F. values as abscissas and the current values as ordinates, we obtain V-shaped curves (Fig. I8), which, in

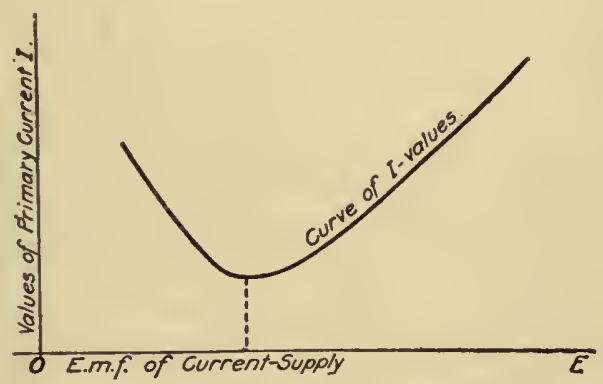

FIG. 18.

consequence of making $\varepsilon$ constant, are independent of the fieldwinding and of the law of saturation.

It is more interesting, however, to take the excitation ampere-turns as abscissas, as in the case of synchronous motors, without distinguishing those due to the series and the shunt windings. This amounts to the same thing as assuming the machine to be separately excited. 


\section{GENERAL DIAGRAMS FOR SYNCHRONOUS MOTORS}

These ampere-turns are determined for each value of the alternating current $I$ by measuring on the diagram (Fig. 13 ), the corresponding segment $C D$, which indicates the reactive current $I_{d}$, and subtracting from this value the ampere-turns corresponding to zero-load which ran give the E.M.F. $\varepsilon$ and the magnetizing ampere-turns produced by the armature, namely,

$$
A_{a}=\left(\frac{K N^{\prime}}{\sqrt{2}}\right) I_{d}
$$

using the + or the - sign according to whether $I_{d}$ is positive or negative, i.e., according to whether the point $C$ be at the right or at the left of $D$.

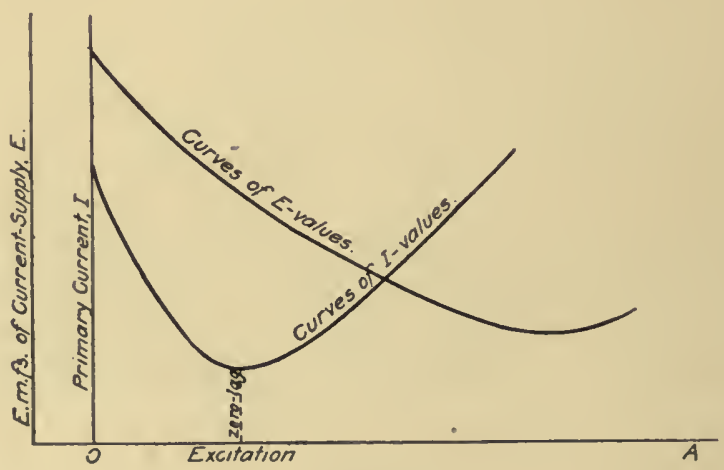

FIG. I9.

Taking the values of the excitation $A$, thus determined, as abscissas, and the corresponding values of $I$ as ordinates, we obtain a V-curve such as shown in Fig. 19, and of which the "bend" corresponds to the load-condition for which there is no lag of the current. If the corresponding values of $E$ are also plotted as ordinates, a second $\mathrm{V}$-curve will be obtained, whose bend is much farther to the right, so that, in the useful portion of the diagram, it consists only of the descending portion of the curve. These curves would be prolonged to the left if we were to consider negative excitations.

A similar pair of $\mathrm{V}$-curves will be obtained for each value of the power-output of the converter.

Upper Limit of Reactive Current. It has already been seen that there is an inferior limit to the reactive current. It also has an upper limit, according to Eq. (23), namely, that value which makes the shunt- 
excitation vanish (a negative shunt-excitation being out of the question). This upper limit value

$$
i_{0}=\frac{A_{0} \sqrt{ } \overline{2}}{K N^{\prime}}
$$

must be greater than the minimum limit, unless the number of turns on the armature is excessive. There is no actual need of an upper limit to the reactive current, because the aim is to reduce it as much as possible, on account of the undesirable effects produced by it on the distribution-system, namely, its increasing the ohmic losses, and its necessitating a higher E.M.F. E. What should determine the value which is to be selected for $i_{0}$ is the condition that, between zero load and normal load, the normal variation of voltage (which determines the range of effect produced by the field-excitation windings), should be that which is suitable for the practical purpose for which the machine is intended, and that which is represented by the current supply-characteristic which has already been defined.

The investigation of this point is facilitated by a new diagram, which will now be considered, and which indicates the lag characteristics of the converter at constant potential.

Lag-Characteristics of Rotary Converters at Constant Potential. Let us suppose that, in some way, the potential difference at the brushes of the converter is maintained constant. The shunt-excitation, which is proportional to this voltage, will also remain constant; and the total ampere-turns which produce this voltage will also remain constant. We will therefore have

$$
A=\text { constant; } A_{d}=\text { constant. }
$$

Hence

or

$$
\begin{gathered}
A_{s}+A_{a}=\text { constant }\left(A-A_{d}\right) \ldots \ldots \\
\frac{\pi k n}{\sqrt{2}}\left(I_{w}-j_{0}\right)+\frac{K N^{\prime}}{\sqrt{2}} I_{d}=\left(A-A_{d}\right) .
\end{gathered}
$$

This formula simply states that, with constant potential, the converter can furnish any secondary current whatever (proportional to $I_{w}$ ) which passes through its series-winding, owing to the automatic decrease of the reactive current, $I_{d}$, as the latter, of itself, takes the value necessary to make up the same total magnetomotive force $A$.

The relation between $I_{w}-j_{0}$ and $I_{d}$ is simply linear. If, therefore, in Fig. 20, the load-values be represented, as previously, by distances 


\section{GENERAL DIAGRAMS FOR SYNCHRONOUS MOTORS}

proportional to $d D=Z I_{d}$ and measured along the reference-line $B Y$; and if the corresponding reactive currents, $I_{d}$, be represented by other segments proportional to $D C=Z I_{d}$; then the locus of the point $C$, at the end of the segment $D C$, is a right line, $Q P$, whose angular coefficient with respect to the point $Q$ as a pole is

$$
\tan \beta=\frac{D C}{D Q}=\frac{\pi k n}{K N^{\prime}} .
$$

This coefficient depends only on the field and armature windings. The right line $Q P$ is easily drawn, in practice by determining the points

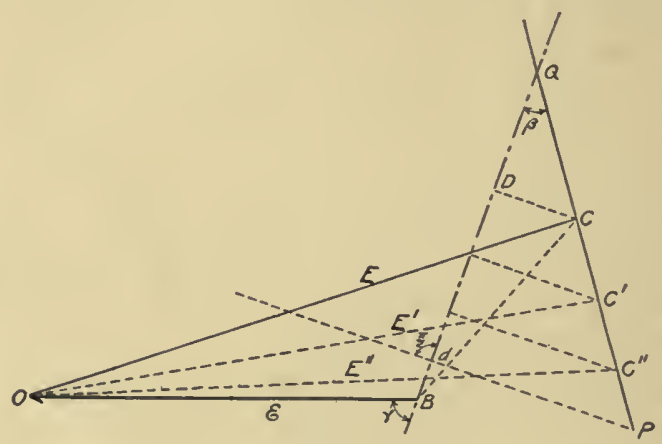

FIG. 20.

$Q$ and $P$ which correspond, respectively, to zero-lag and zero-load: we have

$$
\begin{aligned}
& \text { For the point } P,\left\{\begin{array}{l}
I_{u^{-}}-j_{0}=0 \\
P_{d}=i_{0}=\frac{A-A_{d}}{K N^{\prime}} \sqrt{2} . . .
\end{array}\right. \\
& \text { For the point } Q,\left\{\begin{array}{l}
I_{d}=0 \\
Q_{d}=I_{w}-j_{0}=\frac{\sqrt{2}}{\pi k n}\left(A-A_{d}\right) . .
\end{array}\right.
\end{aligned}
$$

For each value of $\varepsilon$ there exists a value of $A-A_{d}$. This value is obtained from the characteristic curve (Fig. I6), by drawing, from the point $m$, corresponding to this coordinate, the right line ma, making the angle $\delta$ with respect to the axis of $A$ values, the angular coefficient of this line being the resistance of an average turn of the shunt winding, 
Therefore, to each value of $\varepsilon$ there corresponds a right line $P Q$, which is more or less distant from the point $d$; but all these right lines are parallel to each other, having the same angular coefficient, $\rho$, which depends on the windings only. The smaller the number of turns of the series-winding, the farther, evidently, the point $Q$ will be from the point $d$, on the right line $B Y$. When there is no series-winding (in the case of a shunt-wound rotary converter), the right line $P Q$ becomes a right line parallel to $Q Y$, drawn through $P$. Conversely, the decrease of the number of turns in the armature displaces the point $P$ toward the right, on the perpendicular $d P$.

Finally, increasing or decreasing the shunt ampere-turns decreases or increases the difference $A-A d$, and, consequently, brings the right line $P Q$ nearer to or farther from the point $d$. This line passes through the point $d$ when $A d=A$.

Effective Characteristic of Rotary Converter under Load. Following the methods just described, it is easy to study, on the diagram,

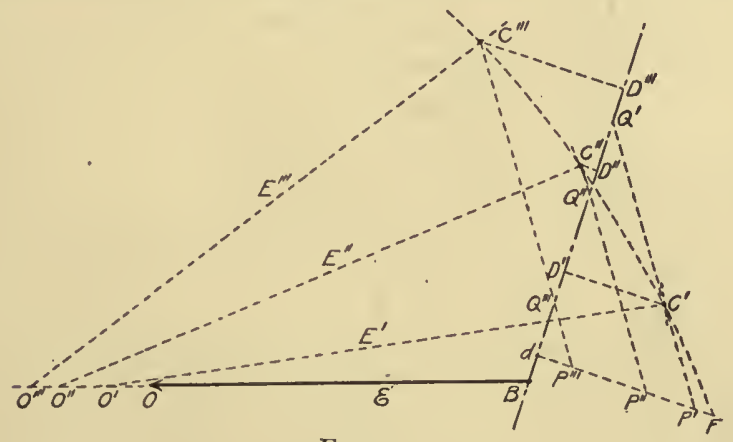

FIG. 2I,

the variations of voltage at the terminals of the converter, as a function of its current-output. It is only necessary to reverse the problem and to find the output corresponding to each voltage, within the limits of variation prescribed or allowed for the voltage.

Let $\varepsilon, \varepsilon^{\prime}, \varepsilon^{\prime \prime}, \ldots$ represent a series of values of the voltage $\varepsilon$. Let these values be represented, in Fig. $2 \mathrm{I}$, by the corresponding distances $B O, B O^{\prime}, B O^{\prime \prime} \ldots$ From the points $O, O^{\prime}, O^{\prime \prime}, \ldots$ let circular arcs be described, with a radius equal to $E$, if the supply E.M.F. is constant, or equal to $E, E^{\prime}, E^{\prime \prime}$. . respectively, if the supply E.M.F. is variable. Let the points of intersection $C, C^{\prime}, C^{\prime \prime}, \ldots$ of these circles with the lag-characteristic line $Q P, Q^{\prime} P^{\prime}, Q^{\prime \prime} P^{\prime \prime} \ldots$ correspond, respectively, to the E.M.F. values $\varepsilon, \varepsilon^{\prime}, \varepsilon^{\prime \prime}, \ldots$ These points will define the cor- 
responding current values, $I_{w}$ and $I_{d}$, of the converter, and, in particular, the loads $d D, d D^{\prime}, d D^{\prime \prime} \ldots$ If the required characteristic has already been drawn by points, for the same values of $\varepsilon, \varepsilon^{\prime}, \varepsilon^{\prime \prime}, \ldots$, the comparison of the two curves will show at once the discrepancies between the values wanted and those which the apparatus can give.

The objection to this diagram is that the circles, in practice, cut the $Q P$ right lines at very sharp angles, and that the points of intersection are somewhat uncertain, i.e., it is difficult to locate them accurately.

In order to make the comparison with greater precision by calculation, the value of $I_{w}$ is calculated, as a function of $\varepsilon$, by Eqs. (2) and (29), namely,

$$
\left\{\begin{array}{l}
Z^{2}\left(I_{d}{ }^{2}+I_{w}{ }^{2}\right)+2 \varepsilon\left(R I_{w}+X I_{d}\right)=E^{2}-\varepsilon^{2}, \ldots \\
\left(\frac{\pi k n}{\sqrt{2}}\right)\left(I_{w}-j_{0}\right)+\frac{K N^{\prime}}{\sqrt{2}} I=A-A_{d} \ldots
\end{array}\right.
$$

These two equations, containing two unknown quantities, $I_{w}$ and $I_{d}$, enable $I_{d}$ to be eliminated, and a solution to be obtained for $I_{w}$ in terms of known quantities, $\varepsilon$ being supposed to have been previously determined. This calculation, though a trifle tedious, presents no difficulties.

The conditions of operation in various cases can be easily predetermined without drawing the curve accurately. Since, from what precedes, the angle of the $P Q$ phase-lines depends on the reactive current $i_{0}$ permitted at zero load, it will be apparent that the choice of values for this current should be made with the idea of making the actual curve agree as closely as possible with the desired curve. The principal practical cases will be investigated from this standpoint.

Application in the Case of Separate Excitation. Let us suppose, at first, for the sake of greater simplicity, that the shunt-excitation is replaced by a separate excitation which is constant. $A_{d}$ is then constant. It is seen, immediately, that if no series-winding is arded, the converter can only give a decreasing voltage as the load increases. The lag-characteristic for all loads is, in fact, a right line, $P Q$, parallel to $B Y$ (Fig. 22); and if we take, on that line, points $C^{\prime} C^{\prime \prime}$, whose distance from $P$ increases, the circles of constant radius, $E$, cut the axis $B O$ at points $O, O^{\prime}, O^{\prime \prime}$, which come nearer to $B$; hence, the voltages proportional to $O B$ are decreasing.

Therefore, whatever may be the reactance and the value selected for the external E.M.F. E, it is not possible to maintain the voltage constant at the brushes on the direct-current side, with a separate excitation, and still less so with a shunt-excitation. It goes without saying 
that the best excitation under these conditions of operation with falling voltage would be one which neutralizes the reactive current $d P$ at all loads; but this mode of operation is not of great practical interest.

It has been proposed to use converters with a falling voltage-characteristic in connection with storage batteries, so as to make the batteries discharge when the load is heavy, and make them charge when the load is light. With the usual method of current-supply this mode of operation would cause variations of 10,20 per cent, or more, in the supply voltage. Dr. F. B. Crocker ${ }^{1}$ has devised an ingenious method of regulating the supply-voltage which enables a wide range of F.M.F. regulation to be obtained in a rotary converter, either with a falling or a rising

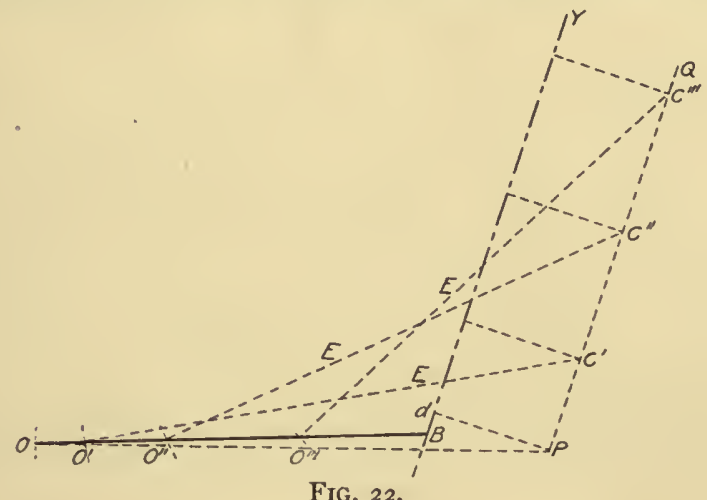

FIG. 22.

characteristic, without affecting the E.M.F. of the source of süpply. The same result is also accomplished by the so-called "split-pole" rotary converter, in another way.

If we add a series-winding which causes the right line $P Q$ to incline toward the left, the variations of $\varepsilon$ will evidently become smaller. We proceed to find the condition which reduces these variations of $\varepsilon$ to a minimum, i.e., which makes the converter give a substantially constant voltage.

It is evidently necessary that the lag-characteristic $P Q$ should coincide as far as possible, within the practical load limits, with a circular arc described from the point $O$ as a center situated at the end of the line $B O=E$. Let $F(23)$ represent the zero-load condition; let $G$ represent the average load, and $M$ the maximum load. A right line

${ }^{1}$ See U. S. Patents to F. B. Crocker, on "Automatic Regulation of Rectifiers and Rotary Converters," No. 891,797. June 23, 1908, and No. I,012,524, Dec. 19, 1911 . 


\section{GENERAL DIAGRAMS FOR SYNCHRONOUS MOTORS}

$s a b$ can be drawn which cuts the circle at two intermediate load-points $a b$ between the extreme points $F$ and $M$, in such a way that this line will not be far distant from the circular arc of any point in its useful portion, $P M$. The segments $d P$ and $d Q$, read off from the diagram (by the proper scale) will determine the currents $i_{0}$ and $I_{w}$ corresponding to zero-load and normal load; and Eqs. (3I) and (32) can be used with these values to deduce the number of constant ampere-turns, $A d$, which are necessary, and also the number of turns $n$ of the serieswindings.

The slight residual differences in the E.M.F. $\varepsilon$ will be obtained by drawing circles of radius $E$ from every point on the right line $P S$ and

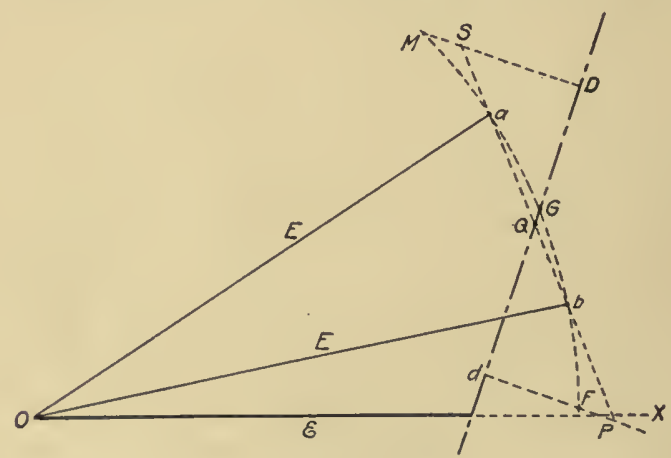

FIG. 23.

taking their intersections with the axis of voltages $B O$. The value of the reactance $X$ affects the precision of regulation, because, the greater the length of the arc $F M$, corresponding to a given variation of load, the more difficult it is to make it coincide with a right line such as $P S$. To improve the regulation, therefore, the segment $d D=Z I_{w}$, which measures the maximum load $I_{w}$, ought to be decreased to a minimum; and, to this end, the reactance (which is the more important factor in the value of $Z$ ) ought to be decreased to the lowest value possible. However, as already seen, the reactance cannot be decreased below a certain value $X_{0}$ without causing the zero-load reactive current $i_{0}$ to increase. It is therefore necessary to take a suitable mean value between $X_{0}$ and zero, which will depend on the circumstances and the conditions of the case. In general, the more the load of the converter is uniform and the less often it runs without load, the more it will be possible to reduce $X$. 
To overcompound the converter the right line $P Q$ should be straightened more, by raising the point $Q$ higher on the right line $B Y$; but the principle always remains the same, namely, to draw the right line $P Q$ in such a way that it shall come as close as possible, at every point, to the desired characteristic, which should itself be drawn first.

Application to the General Case of Self-Excitation. In the general case of self-excited converters, the solution is nearly the same, but it is complicated by the fact that the shunt-excitation varies with the direct-current voltage. Hence, instead of only one right line $P Q$ to indicate the lags, there will be a series of parallel right lines corresponding to various values of $\varepsilon$.

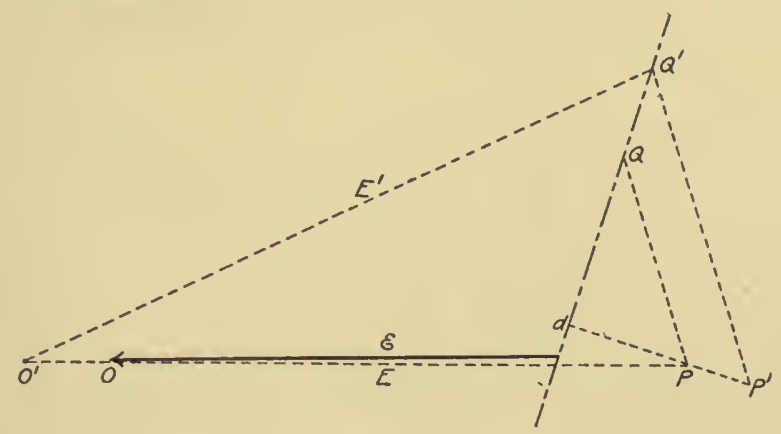

FIG. 24.

In the case of constant potential regulation, the method of determining the excitation-windings and the reactance of the circuit remains exactly the same as given in the preceding paragraph, since the shuntwinding must, by hypothesis, produce constant excitation. But the small variations of $A_{d}$ have to be considered in the final calculation of the differences of voltage due to the imperfection of the regulation.

For values of $\varepsilon$ lower than the normal value, the shunt-excitation decreases of itself and, consequently, the right line $P Q$ comes closer to $d$, remaining parallel to itself. On the other hand, this line receres from $d$ when $\varepsilon$ increases. It is easy to see, in Fig. 24, that this effect tends to increase the voltage-differences as compared with these differences when the shunt-excitation is constant.

When the machine is to be overcompounded the zero-load and the normal load no-lag conditions determine, as before, the reactive 
ampere-turns, for zero-load, $A_{0}$, and, therefore, the shunt and series windings

Let $\varepsilon$ and $\varepsilon^{\prime}$ represent the voltage-values corresponding to these two load-conditions, according to the excitation-curve. The laglines corresponding to all the intermediate values are parallel, and are comprised between two lines which are determined by Eq. (29) when the right-hand member is made equal to $A_{1}-A_{0}$ and to $A^{\prime}$, respectively. These lines pass through $P$ and $P^{\prime}$ respectively.

In order to obtain effective overcompounding, i.e., to have $\varepsilon^{\prime}>\varepsilon$, it is necessary that the point $O$ should not be very far distant from $P$ on the right line of reference $B Y$. This means, simply, that it is necessary to have either a powerful series-winding, or else a large reactive current with zero-load. The first of these two ways appears to be the

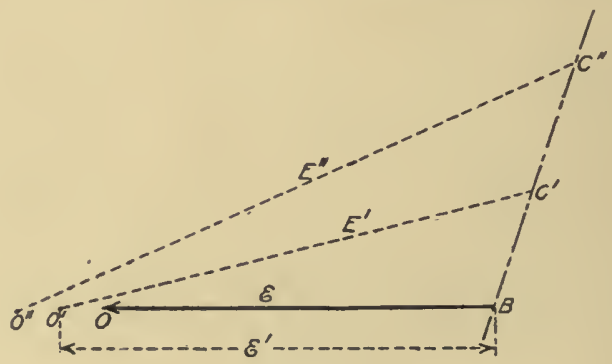

Fig. 25.

preferable one; but inasmuch as it is always desirable to increase the inclination of the right line $B Y$ to the right in order to decrease $\varepsilon$, it is necessary to resort to both plans to obtain overcompounding.

Regulation of Supply E.M.F. by Compounding of the Generator. As already stated, it is possible, theoretically, to do away with the reactive current at all loads by using, as the source of current-supply for the converter, a suitably compounded alternator, giving an E.M.F. E, which increases with the load in such a way that the point $C$ is always displaced on the right line of reference $B Y$, while $\varepsilon$ remains constant or else increases, according to the results desired. It is interesting to know how the excitation should be effected in such a case.

If it be required to have the direct-current voltage, $\varepsilon$, constant, the excitation will evidently be that of a simple shunt-winding; and its value is easy to determine by the condition that it must give exactly the voltage $\varepsilon$, with zero-load, since there is not to be any reactive current. 
If, on the other hand, it be required to have the voltage rise with the load, the excitation must be compound. The shunt-winding will still be such that it, alone, gives the no-load F.M.F. $\varepsilon$, and the serieswinding will be such that it will produce the additional ampere-turns necessary to give the E.M.F. $\varepsilon^{\prime}$ required with the load $I_{w}$. As already stated, in a case like this, it is desirable to decrease the reactance $X$, and the lag $\theta$, to a minimum.

Regulation of Voltage by Varying the Reactance $\mathrm{X}$ in the Circuit. The two windings, in this case, will be determined in the same way as in the preceding case, since the voltage of the alternating current supplied to the converter is still regulated by outside means without causing lag in the current inside the machine. The excitation will be that produced by a simple shunt-winding when constant voltage is wanted, and that produced by a compound-winding, when a rising voltage is wanted. The shunt-winding will be determined by the voltage required with no load, and the series-winding will be determined by the voltage required with full load.

Possibility of Suppressing the Shunt-Winding. It is not absolutely necessary to use either a separate exciting winding, or else a shunt winding, as has been supposed hitherto; because, by making the number of armature-winding turns greater and decreasing the airgap, it is always possible to obtain the necessary no-load excitation, merely by the effects of the armature-reaction. Eys. (2I) and (22), in that case, reduce to the following forms:

$$
\begin{gathered}
\frac{\pi k n}{\sqrt{2}}\left(I_{m}-j_{0}\right)=A_{m} . \\
\frac{K N^{\prime}}{\sqrt{2}} i_{0}=A_{0} .
\end{gathered}
$$

It is not possible to obtain constant voltage or rising voltage, in the direct-current circuit with this method any more than when a shuntwinding is used, without series-winding. This method has, to some extent, the self-regulating properties of transformers, but to a degree which is inadequate. owing to the greater reluctance of the magnetic circuit.

On the other hand, the high values which the reactive currents must then be allowed to attain have the great objection of causing increased heating of the armature by increasing the total current in 


\section{GENERAL DIAGRAMS FOR SYNCHRONOUS MOTORS}

the armature; and also of increasing the lag of the current in the supply-circuit, thereby reducing the output-capacity of the generator, increasing the voltage drop in the line, etc. In a word, this method has all the faults and objections due to heavy reactive currents, in addition to which the commutation of the machine is much less satisfactory, there being more tendency to sparking, owing to the shortened air-gap.

This method, which was used by certain concerns, has now been abandoned altogether. It is referred to here merely to show the ease with which it is possible to calculate the excitation for the conditions. obtaining in this case as well as in the preceding cases. This method has one advantage, however, that of making the satisfactory performance of the converter more independent of the form of the E.M.F. curve of the generator.

Conclusion. It has been shown, in what precedes, that the vectordiagram for synchronous motors, when referred to two axes, lends itself, as the result of natural simple extensions, to the study of the rotary converter and enables its conditions of operation to be analyzed for the most varied cases. This method of analysis serves to render more intelligible the very complex problem involved in the voltage regulation of the rotary converter, which is, perhaps, the most complicated problem presented in connection with alternating-current machinery.

The methods set forth in the preceding pages not only enable the phenomena to be foreseen qualitatively, but it also enables them to be calculated numerically, by combining the diagrams, or the equations which are derived from them, with the data obtained from experiments or tests. 


\section{CHAPTER III}

\section{STABILITY OF OPERATION OF ROTARY CONVERTERS}

WHEN in operation, rotary converters sometimes produce variations of voltage above and below their normal voltage; and they may also, at times spark, or even flash, at the commutator. These phenomena might be attributed to changes of frequency due either to variations of angular velocity of the prime mover driving the alternator which supplies current to the converters, or else due to oscillations of its speedgovernor.

To eliminate these two causes of irregular operation, the following experiment was tried: the rotary converter was driven as a motor by current taken from a storage battery, which also excited the shuntfield winding. Three-phase currents taken from the A.C. side of the machine were passed through a raising transformer to raise their voltage. The voltage was then lowered by step-down transformers and the energy transmitted through them was absurbed by means of three identical rheostats. The arrangement was equivalent, practically, to connecting a three-phase inductive resistance to the secondary of the first transformer (Fig. 26). It was then observed that, on increasing gradually the loat on the converter, a critical point was reached where its speed began to oscillate between limits which were all the farther apart the more the load was increased.

To explain this phenomenon, let us note that the converter was sending reactive currents into the circuit, and that these reactive currents tended to weaken the magnetic field. Now in the case of a load which is below a certain value(which we will term the limiting or critical load) the strength of this reactive current should decrease when the speed of the rotary converter increases, and reciprocally, it should increase when the speed decreases. The contrary effect should be produced when the load exceeds this critical load.

To demonstrate this, let us refer to a transformer diagram of the kind which takes magnetic leakage into consideration. It is known (Fig. 27 ) that if $O Y$ is the axis of E.M.F.'s the end of the vector $(O M)$ 


\section{GENERAL DIAGRAMS FOR SYNCHRONOUS MOTORS}

representing the strength of the primary current describes a semicircle which cuts the axis of $x$ at two points, $A$ and $B$, such that $O A=i_{d v}$ represents the zero-load magnetizing current, and $O B=\frac{i_{d v}}{\sigma}$, where $\sigma=\frac{\mathrm{I}}{v_{1} v_{2}}$, in which $v_{1}$ and $v_{2}$ are the leakage coefficients for the two windings of the transformer.

By hypothesis, the voltage of the source of current-supply is constant. Therefore, an increase of speed of the converter, by causing an increase in the frequency, will bring about a decrease of the magnetiz. ing current corresponding to zero-load. We will have a new circle,

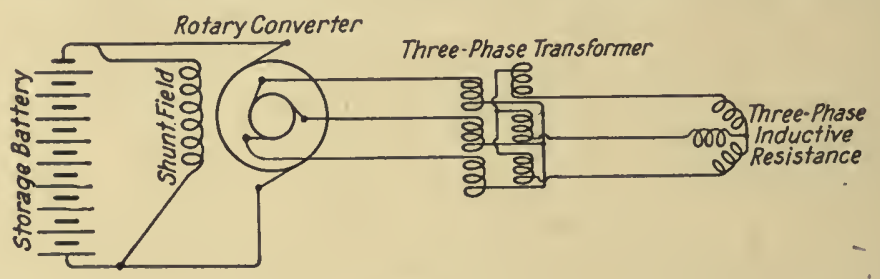

FIG. 26.

$A^{\prime} B^{\prime}$, whose diameter will be $i_{d v}^{\prime}\left(\frac{\mathrm{I}}{\sigma}-\mathrm{I}\right)$. The two circles will intersect each other at a point $M$, which is on the line drawn, from $O$, tangent to one of the circles.

It is seen, at once, on the diagram, that the right line $O N$, which represents the active current in relation to the primary current $O M$, indicates the critical load already mentioned. In fact, for the value $O H$, corresponding to a load which is lighter than the critical load, i.e., which makes $O K<O N$, it is seen that the reactive current has decreased from the value $O L$ to the value $O L_{1}$, as the result of a speed-increment. On the contrary, for the current $O P$, corresponding to a load which is heavier than the critical load, i.e., which makes $O Q>O N$, the reactive current has increased from $O R$ to $O R_{1}$. It is now easy to analyze the phenomena observed.

In the first place, when the load was lighter than the critical load of the machine, an increment of speed produced a decrease of the reactive current, and, consequently a decrease in demagnetizing action; and, since the converter was running as a direct current motor, with constant shunt-field-excitation, its total excitation-flux was increased. Therefore, the E.M.F. on the D.C. side being constant, the speed of 
the converter had to decrease, in order to maintain the same induced E.M.F. The converter therefore varied in speed in order to maintain the induced E.M.F. constant.

In the second place, when the load was heavier than the critical load of the machine, an increase of reactive current was produced by a speed increment. Hence, there was a decrease in the total excitation flux. The speed of the motor therefore tended to increase until the current supplied from the battery was limited by the resistance of the circuits. As the armature of the converter was then in a state of

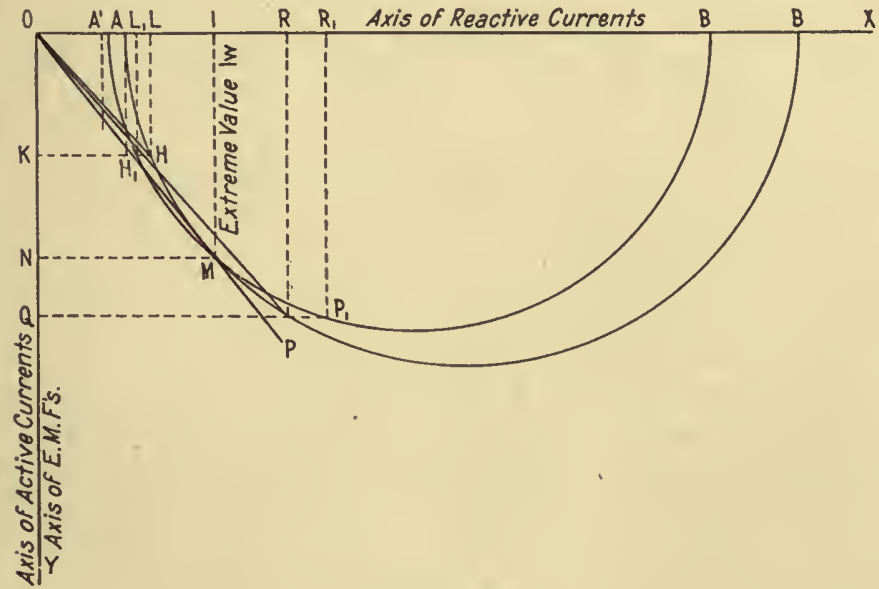

FIG. 27.

unstable dynamic equilibrium its speed soon began to decrease; and thus speed-oscillations were produced.

In practice, therefore, it is desirable that the load put on the rotary converter should not exceed the critical load above mentioned.

Let us see, now, what can be done to raise this load-limit. We will use the same diagram as before (Fig. 27).

We see readily that what is necessary is to increase the vector $O N$. This may be accomplished in different ways:

First, if the frequency is decreased, then, for a given voltage, other things being equal, the magnetizing current will have to be increased. Therefore the diameter of the circle corresponding to $i_{d v}\left(\frac{\mathrm{I}}{\sigma}-\mathrm{I}\right)$ will be increased, and, consequently, the point of contact $M$, of the line 


\section{GENERAL DIAGRAMS FOR SYNCHRONOUS MOTORS}

which is tangent to this circle, is farther away from the axis $O x$; hence $O N$ is increased.

Second, if the coefficient of self-induction of the transformers is decreased, while the other quantities remain unchanged, the magnetizing current corresponding to zero-load will increase; hence $O N$ is increased.

Third, if the magnetic leakage is decreased, then $\frac{I}{\sigma}$ increases, and, consequently, the diameter of the circle also increases, causing as before the point $M$ to move farther away from $O x$, and increasing $O N$.

To sum up, then, the limiting load on a rotary converter can be increased: I, by diminishing the frequency of the A.C. source; 2, by reducing the self-induction of the transformers; 3 , by reducing the magnetic leakage.

These facts have been all verified experimentally.

II. Let us now consider the rotary converter as a transformer of alternating into direct current.

The rotary converter used in the previous experiments was operated regularly at full load with the transformer connected to a suitable source of alternating current supply.

In order to regulate the voltage of the direct current produced by the converter, an (A.C) induction-regulator having a high coefficient of magnetic leakage was included in the primary circuit of the transformer. The rotary converter then became subject to speed oscillations of long period as soon as its load was increased to half the load which was carried without the induction-regulator. To explain this fact we shall show that every speed-increment of the rotary converter causes an increase in the difference of potential at its terminals.

Let us refer again to the converter-diagram. Let $O A$ (Fig. 28), represent the difference of $A$.C. potential at the terminals of the converter; $O C$, the supply-voltage which, in this case, is constant; 0 , the phase-angle between these two F.M.F.'s; $A D$, the active current, including the current corresponding to friction, eddy currents, etc., $D C$, the reactive current, counted as positive on the right side of the axis of currents $B Y$, and counted as negative on the left side thereof.

Every increment of speed of the converter causes a decrease of the angle 0 . Consequently the diagram shows that, $O C$ being constant, the active current $A D$ will decrease (to $A D^{\prime}$ ) and the reactive current $(D C)$ will increase (to $D^{\prime} C^{\prime}$ ). Moreover, as in the 
case of synchronous motors, there appears an E.M.F. which is $\frac{\pi}{2}$ in advance of the E.M.F. $\varepsilon$ and which is equal to $\omega L^{\prime} d\left(I_{w} w^{*}\right)$. But $\Delta I_{w}$ is itself negative (being equal to $A D^{\prime}-A D=-D D^{\prime}$ ); therefore, this E.M.F. $(A F)$ is $\frac{\pi}{2}$ behind the E.M.F. $\varepsilon$. This E.M.F. here tends to diminish the increment of $I_{d}$, but it increases $I_{w}$ still more.

The ampere-turns by which the E.M.F. $\varepsilon$ is induced are equal to $A_{d} I \frac{K N^{\prime}}{\sqrt{2}} I_{d}$, in which $A_{d}$ represents the ampere-turns due to the constant excitation. It is seen at once, that whether the point $C$ be at the right or at the left of the line of reference $\left(B Y^{Y}\right)$ for current-

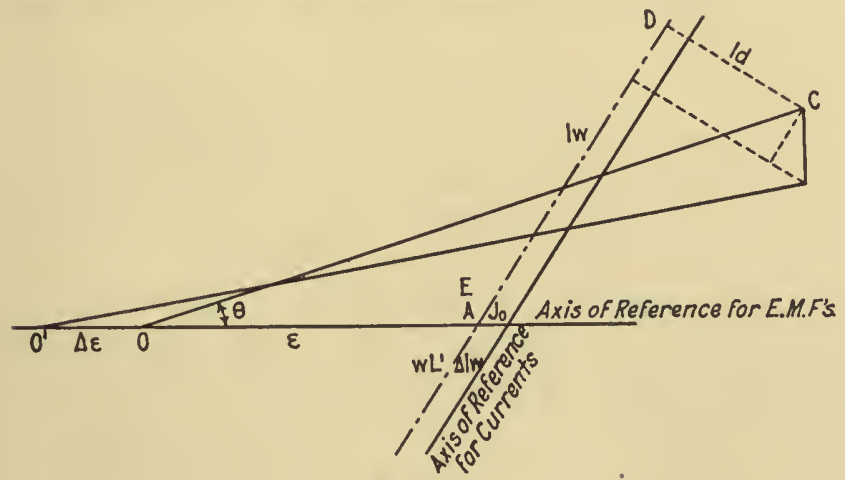

FIG. 28.

values, the value of the excitation for an increment of $I_{d}$ is always equal to $A_{d}+\frac{K N^{\prime}}{\sqrt{2}} d\left(I_{d}\right)$; consequently, the excitation will increase in all cases, and so will the E.M.F. $\varepsilon$.

The power developed at the shaft of the rotary converter, when in normal operation is equal to

$$
P=\varepsilon\left(I_{w}-\frac{\varepsilon}{\xi R}\right) .
$$

When the increment of speed occurs, the increase of power developed at the shaft is equal to

$$
\Delta P=\Delta\left(\varepsilon I_{w}\right)-\frac{I}{\xi R} J\left(\varepsilon^{r}\right)+\omega L^{\prime}\left(\Delta I_{w}\right) I_{d}
$$

In order to have stability of operation we should have $A P<0$. 


\section{GENERAL DIAGRAMS FOR SYNCHRONOUS MOTORS}

It is seen, from the diagram, that, at light loads, $\theta$ being then small, the increment of $I_{d}$ is small in comparison with the increment of $I_{w}$, whereas with heavy loads, 0 being then large, the reverse is the case.

The observed phenomenon can now be explained.

If the voltage at the terminals of the converter remained constant the torque would decrease when the speed increases. But each increase of voltage causes an increase of torque. At light loads the first effect is greater than the second; therefore, since the torque decreases, there is an automatic regulation of the speed.

Beyond a certain load, the second effect preponderates, and there is an increase in the torque. The converter therefore continues to gain in speed until the torque ceases increasing. It is then in a state of unstable equilibrium and it then loses speed until the torque ceases decreasing.

Experiment shows that the amplitude of the speed-oscillations increases rapidly with the load and that it then becomes impossible for the machine to operate. The oscillations can be diminished by taking advantage of the fact that the torque of the converter diminishes with its excitation. If we take a compound excitation comprising a shunt-winding connected to the terminals of the converter and a serieswinding connected differentially, it will be seen that whenever there is an increase of voltage at the terminals of the converter, there will be an increase of the load-current. This current, acting upon the series-winding, will cause an increase in the series-excitation which is opposed to the shunt-excitation. Therefore the total excitation will decrease, and the torque will also decrease. These different effects can be calculated and adjusted so as to balance or overbalance the increase of torque. Actual test shows that this can be done, and that the converter can be made to carry full load even when the inductionregulator is used, if the series-winding is reversed. It appears, therefore, that, in the case of a rotary converter which is transforming direct current into alternating current, it is desirable to have a compound winding in which the shunt and series excitations add themselves, or act cumulatively.

On the other hand, a rotary converter serving to transform alternating current into direct current should have a compound winding in which the series and shunt excitations act differentially. 


\section{CHAPTER IV}

\section{OPERATION OF SEVERAL ROTARY CONVERTERS IN PARALLEL}

THE parallel working of rotary converters is a much more difficult matter than the parallel working of ordinary generators, owing to the fact that the load has a tendency to distribute itself unequally, without any correcting action in the case of rotary converters.

Any increase of speed occurring in a converter causes the alternating current to lead in phase with respect to the induced E.M.F. This strengthens the magnetic field of the converter, and, consequently, causes the induced E.M.F. to be still further increased. Therefore, there is a tendency to unstable operation. To avoid the unequal distributions of load in rotary converters which are connected in parallel, they not only require careful attendance, but their design and construction demand much care, because the various converters intended for parallel working should, as nearly as possible, have the same armature-resistance and the same magnetic reluctance in the magnetic circuits of the field-magnets; in fact, the magnetic leakage and the magnetization curve should be substantially the same for all the machines.

It is often necessary to give up the attempt to compound the rotary converters and to use only shunt-wound rotary converters, for parallel working. In any case when compound windings are used it is almost always necessary to adjust these windings after the machines are in position, in order to make them run in balance at all loads. It is understood, of course, that an "equalizer" connection is necessary between the different machines as in the case of all other direct-current compound-wound dynamos connected in parallel.

It is more difficult still to make the converters run in balance when, instead of being coupled directly, they are coupled through feeders supplying current to a line which is common to all of them, as, for instance, a section of trolley or of third-rail conductor, in electric railway operation. In such cases it has been found necessary to 
reduce the voltage-drop in the feeders to a small proportion, i.e., 2 to 3 per cent of the normal working voltage.

\section{INHERENT OSCILLATIONS OR PUMPING OF CONVERTERS CONNECTED IN PARALLEL}

Speed-oscillations can be produced in rotary converters, same as in synchronous motors, by periodic speed-variations in the alternators supplying them with current, when these alternators are driven by steam engines whose angular velocity varies at different portions of each piston-stroke; and they may also be produced by sudden changes or fluctuations of load. These speed-oscillations in rotary converters may even have a much greater amplitude than in other machines, because they are not opposed by a contrary variation of the torque acting upon the armature-shaft. As already stated, when the speed of a converter increases, its current is thrown out of phase and becomes leading with respect to the induced E.M.F. This causes the magnetic field to become stronger and the induced E.M.F., to rise; consequently, the secondary (direct current) output increases. The primary (input) power increases, however, in substantially the same proportion; hence, there is no other variation in the torque acting upon the armature shaft than that which can result from the variations of the losses with the load; and this effect is always quite small in comparison with the inertia of the armature.

The amplitude of the oscillations may, therefore, be very large; sometimes it is sufficient to make the converter "fall out of step." More often, its effects are made evident by fluctuations in the (D.C.) E.M.F. of the converter-which have amounted to as much as 30 per cent - and also by sparking at the commutator.

The E.M.F. fluctations are easily explained by the variations of speed and reactive current which occur simultaneously with them. The sparking is due to a much more complicated action. Owing to the inertia of the armature, every increase in speed, causing additional kinetic energy to be stored in the armature-mass, makes the primary (input) power greater than the secondary (output) power; and every decrease in speed, causing the armature to lose some of the kinetic energy stored in its mass, produces the contrary effect of making the secondary power greater than the primary power.

In both cases the transverse armature-reactions produced by the primary and secondary active currents no longer balance each other, and the axis of the resultant magnetic field oscillates from one side of 
the line (diameter) of commutation to the other; and, as the brushes cannot follow these oscillations, sparking occurs, just as it does in the case of a dynamo whose brushes are not at the right position for sparkless running.

To overcome or diminish these difficulties it is desirable to avoid as much as possible using alternators driven by engines whose speed is unsteady or irregular, and to use prime movers giving a speed which is as uniform and constant as possible. However, speed-variations may be produced in the best-regulated steam engines and turbines, by sudden and large changes of load. For this reason it is desirable to apply the remedy to the converter itself, even though, in consequence, a small fraction of the useful energy may be lost.

This remedy, which is quite simple, is well known since the "dampers" of Hutin \& Leblanc have been introduced. The principle involved is that the speed-oscillations themselves cause eddy currents to be produced either in the pole-pieces or else in "damping circuits" placed suitably around or about the pole-pieces.

A noticeable damping effect will already be obtained by merely making the pole-pieces of solid annealed wrought iron. The damping effect produced is not always sufficient, however; and as eddy currents are also produced in such pole-pieces in consequence of the irregularity of the revolving field caused by the two-phase or three-phase currents employed, the loss of energy due to these eddy currents is not always utilized in improving the stability of running of the converter.

In attempting to use laminated pole-pieces with the object of eliminating these eddy current losses and of improving the efficiency of the converter, it was soon found that the stability of operation of the converter was, thereby, made altogether insufficient. The proper stability was restored to the converter by placing copper or brass rings around the pole-pieces, or else simply by placing the field-excitation windings on copper spools or forms constituting a closed electric circuit around the field-cores and poles.

These "damper-circuits" are adjusted in such a manner as to reduce as much as possible the energy-loss produced by the currents induced in them, while at the same time obtaining the damping effect required. For example, Mr. Parshall has, in this way, adjusted the damping of several converters of $900 \mathrm{KW}$. capacity by connecting them in parallel on an artificial line. Even when supplied with currents by an alternator whose speed was irregular they operated satisfactorily with a loss of 3 per cent in the reactances interposed between them. 
The loss in the damping circuits was then gradually decreased from 3 per cent to 0.6 per cent only, without the converters falling out of step. Experience shows that, as a general rule, the stability of the converter is better when the supply-current is lagging than when it is leading, probably because the induced E.M.F. is higher with leading currents. Much better results will be obtained, both in respect to the damping of speed-oscillations and in respect to ease of starting, by using the Leblanc dampers in their most perfect form, namely, in the form of grids consisting of copper bars or rods imbedded in the polepieces (parallel to the shaft) and connected to copper plates at both ends. (See part I, Fig. 62.) In most cases, however, to reduce the cost, a simpler form of damper is used.

\section{USE, OF. ROTARY CONVERTERS FOR TRANSFORMING DIRECT INTO ALTERNATING CURRENT}

Since rotary converters are reversible machines they can be employed, in certain cases, for producing alternating currents from direct current.

There may be cases where a small portion of the output of a directcurrent generating plant must be transmitted to a considerable distance. In such cases the power to be transmitted can be converted into hightension three-phase alternating currents which, after being transmitted to the receiving end, can be utilized there as alternating currents or else can be reconverted into direct currents. Again, in certain directcurrent installations, it may be desirable to convert a portion of the power into three-phase alternating currents for operating certain apparatus, such as, for instance, for supplying induction-motors, in cases where commutator-motors would not be allowable. It is also possible and it may be desirable in certain cases to produce polyphase alternating currents by means of a converter supplied with direct current from a storage battery.

In such cases the characteristics and the performance of the rotary converter are materially different from what they are in the cases previously considered, being similar in all respects to those of direct current motors.

In the first place the speed is not constant, but it depends upon the field-excitation, owing to the fact that the E.M.F. induced in the directcurrent armature-winding must always be substantially equal to the F.M.F. of the source of (D.C.) current, less the ohmic drop. Hence, when the field-excitation is made stronger the speed decreases, and vice versa. 
Moreover, the armature-reactions produced by the reactive current produce the same effect on the resultant total field-excitation. The alternating current produced by the converter, in such a case, generally lags behind the (A.C.) E.M.F., hence it weakens the magnetic field, and this, instead of causing a falling off in voltage, causes an increase in speed. If the A.C. circuit happens to contain a considerable amount of inductance, the reactance will increase with the frequency (due to the increase of speed); and the lag will increase with the reactance. The demagnetizing effect will therefore be increased. This will cause a further increase of speed, a further increase of frequency, and again a further increase of reactance; and the consequence of this cumulative process may be that the converter will " run away" or attain an excessive speed. It is therefore necessary, in such cases, to take special precautions, even including the use of safety-devices such as automatic safety stops, set so as to operate to open the (D.C.) supply-circuit whenever the converter speed exceeds a certain limit.

This want of stability can be avoided, and the converter can be made to operate under synchronous conditions, as before, by simply connecting to it (in parallel with its A.C. circuits), an alternator which is driven at constant speed by an engine or motor, and which has sufficient power to withstand any tendency to be itself driven as a motor by the current produced by the converter. In that case, the alternator regulates the frequency, and it "sets the step" for the converter, as it were, also furnishing to the converter the reactive current necessary to bring back the magnetic field to the normal value, whatever may be the excitation due to the field-windings.

Other Special Applications of Converters. As already stated, rotary converters may be used as motors for developing mechanical power equal to a poriton of the electric power supplied to them either on the A.C. or the D.C. side. In such a case, however, the acttve currents entering and leaving the converter will no longer balance each other, the difference between the energy-values which they represent being converted into mechanical energy. The "transverse" reactions no longer offset each other, and the heating effects are increased.

This case can also be studied by the graphical methods previously presented, because the diagram representing the conditions will be simply intermediate between the two diagrams shown in Figs. 27 and 28 , correspcnding, respectively, to a synchronous motor and to a converter. All that is needed is to change the lengths of the segment $O B$, making it equal to $\omega L^{\prime}\left(I_{w}-I_{w^{\prime}}\right)$ instead of $\omega L^{\prime} I_{w}$, in 
which $I_{w}^{\prime}$ will represent the reactive current transformed into direct current.

It is also possible to use a rotary converter as a partial generator, by applying mechanical power to its shaft. This mechanical power is added to the electric power applied to the converter from the electrical source (A.C. or D.C.) supplying it. The power-output of the converter, in such a case, will be equal to the sum of the two power-inputs less the conversion-losses. This case can also be discussed easily by means of the preceding diagram.

There is still another case, namely, where a rotary converter is used entirely as a generator, for generating both alternating and direct currents. In such a case the machine is not operating as a rotary converter at all, but as a "double-current" or "omnibus" dynamo. The machine is then a particular kind of alternator and its characteristics should be studied as such. The reactions due to the two kinds of current no longer compensate, but add, themselves; hence there will be sparking at the brushes unless their position be shifted with the load; and the heating effects will be increased instead of decreased, as compared with the operation of the same machine as a rotary converter.

Phase-Converters. Instead of making a rotary converter produce direct current when supplied with three-phase current, it could be made to supply two-phase alternating current. The reactions occurring in such a machine would be very interesting, because they would be much more complicated than in the case of ordinary rotary converters. In fact, the (two-phase) currents generated by the machine would no longer have a definite, fixed, phase-relation with the E.M.F., as in the case of the direct current, but they would have a lag which would vary with the reactance in the external circuit.

It could again be seen easily, by the same reasoning as before, that since the power applied to the machine, and the power delivered by it have nearly the same values, the transverse reactions due to the active current must practically offset or balance each other, leaving only the direct reactions due to the reactive currents. The latter can be evaluated separately and we can thus obtain, for each value of the power output and of the lag in the secondary circuit, the resultant magnetomotive force produced by the reaction of the armature on the magnetic circuit. 


\title{
CHAPTER V
}

\section{VOLTAGE RATIO IN SYNCHRONOUS CONVERTERS WITH SPECIAL REFERENCE TO THE SPLIT-POLE CONVERTER}

\author{
Résumé of paper by C. A. AdAMs, Proc. A.I.E.E., June, I 908
}

Tre author of this paper shows how the ratio of the D.C. to the A.C. voltage of a synchronous converter may be varied by means of the split pole, without introducing seriously large harmonics into the counter E.M.F. and current. Incidentally a new method is employed for calculating the E.M.F. between adjacent commutator brushes-the brush E.M.F. - and the corresponding E.M.F. between collector rings-the tap E.M.F.-for any given flux distribution.

The elementary E.M.F. induced in a single conductor cutting through the gap-flux at constant speed is proportional to the density of the flux, and when plotted, will obviously have the same shape as that of the gap-flux curve. It may be expressed thus:

$$
e^{\prime}=a_{1} \mid \begin{gathered}
\sin \omega t+q_{a 3} \sin 3 \omega t+q_{a 5} \sin 5 \omega t+\ldots \\
+q_{a m} \sin m \omega t+\text { etc. } \\
q_{b 1} \cos \omega t+q_{b 3} \cos 3 \omega t+q_{b 5} \cos 5 \omega t+\ldots . \\
+q_{b m} \cos m \omega t+\text { etc. . . . . . }
\end{gathered} .
$$

where $\omega=2 \pi n$ and $n$ is the fundamental frequency.

The brush voltage which is proportional to the area of the $e^{\prime}$ curve between brushes, is

$$
E_{d c}=N \frac{2}{\pi} a_{1}\left[\mathrm{I}+\frac{1}{3} q_{a_{3}}+\frac{1}{5} q_{a_{5}}+\frac{1}{7} q_{a_{7}}+\frac{1}{9} q_{a 9}+\text { etc. }\right] .
$$

or

$$
E_{d c}=E_{1}\left[\mathrm{I}+\frac{1}{3} q_{a 3}+\frac{1}{5} q_{a 5}+\frac{1}{7} q_{a 7}+\text { etc. }\right]
$$

where $N$ is the number of conductors in series between brushes.

The tap E.M.F. is the geometrical sum of the E.M.F.'s of the several coils connected in series between taps. The E.M.F. of each 
coil is in turn the geometrical sum of the E.M.F.'s of its two sides, which E.M.F.'s may or may not be in phase according to whether or not the coil pitch is equal to the pole pitch. The fundamental E.M.F.'s of the two sides of each coil will differ in phase by the deficiency in coil pitch measured in electrical degrees, and the fundamental E.M.F.'s of the coil sides in two adjacent slots will differ in phase by the electrical angular pitch of the slots. In each case the phase difference between the $m$ th harmonics is $m$ times that between the fundamentals.

In calculating the tap E.M.F. the author introduces the "differential factor," the ratio of the resultant or geometrical sum to the arithmetrical sum of the various E.M.F.'s.

Let $N_{s p}=$ slots per pole, and $p^{\prime}$ the number of belt spans per electrical circumference $\left(p^{\prime}=2\right.$ for diametral connection and 3 for three-phase delta or six-phase double delta); then $\frac{\pi}{N_{s} p}=$ the phase difference between the fundamental E.M.F.'s of two adjacent slots, $\frac{m \pi}{N_{s p}}=$ the phase difference between the corresponding $m$ th harmonics and $\frac{2 N_{s p}}{p^{\prime}}=$ slots per belt. The total phase rotation (or combined phase displacement) of the $m$ th harmonic in all the slots of the belt will be

$$
\frac{m \pi}{N_{s p}} \times \frac{2 N_{s p}}{p^{\prime}}=\frac{2 m \pi}{p^{\prime}}
$$

and the resultant of the $\frac{2 N_{s p}}{p^{\prime}} m$ th harmonics is proportional to $2 \sin \frac{m \pi}{p^{\prime}}$. By the same constant a single $m$ th harmonic is proportional to $2 \sin \frac{m \pi}{2 N_{s p}}$, and the arithmetical sum of the $\frac{2 N_{s p}}{p^{\prime}} m$ th harmonics is

$$
\frac{2 N_{s p}}{p^{\prime}} \times 2 \sin \frac{m \pi}{2 N_{s p}} .
$$

The differential factor for the $m$ th harmonic of the belt E.M.F.; or the belt-differential factor for the $m$ th harmonic is then

$$
k_{d m}=\frac{p^{\prime}}{2 N_{s p}} \frac{\sin \frac{m \pi}{p^{\prime}}}{\sin \frac{m \pi}{2 N_{s p}}}, \ldots . . .
$$


and that for the fundamental

$$
k_{d 1}=\frac{p^{\prime}}{2 N_{s p}} \frac{\sin \frac{\pi}{p^{\prime}}}{\sin \frac{\pi}{2 N_{s p}}}
$$

The ratio $k_{d m} \div k_{d 1}$ is the relative reduction of the $m$ th harmonic by belt-differential action; it may conveniently be called the beltreduction factor; and is

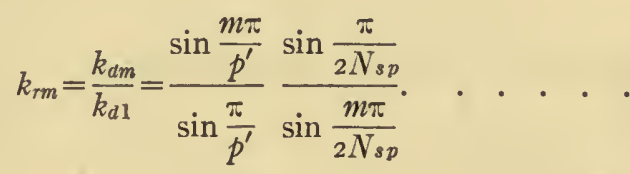

The first part of $k_{r m}$, namely, $\frac{\sin \frac{m \pi}{p^{\prime}}}{\sin \frac{\pi}{p^{\prime}}}$, is independent of $N_{s p}$ and is equal to $\mathrm{I}$, except for $m=3,9$ or $\mathrm{I} 5$, when it is zero for $p^{\prime}=3$, and 2 for $p^{i}=6$.

The following table gives the value of the second part of $k_{r m}$, which is equal to $k_{r m}$ except for the cases above cited.

\section{TABLE II}

\begin{tabular}{|c|c|c|c|c|c|c|c|c|c|c|c|}
\hline$N_{s p}=$ & 8 & Io & 12 & 14 & I6 & I 8 & 20 & 22 & 24 & 26 & $\infty$ \\
\hline$m=3$ & 0.351 & 0.344 & 0.340 & 0.338 & 0.3368 & 0.3362 & 0.3358 & 0.3355 & 0.3353 & 0.3351 & $\frac{1}{3}$ \\
\hline$m=5$ & 0.235 & 0.222 & 0.214 & $0.2 \mathrm{IOI}$ & $0.208 \mathrm{I}$ & 0.2062 & 0.2051 & 0.2042 & 0.2034 & 0.2029 & $\frac{1}{5}$ \\
\hline$m=7$ & 0.199 & 0.1755 & 0.1646 & 0.158 & 0.154 & 0.152 & 0.150 & 0.149 & 0.1477 & o. 1467 & \\
\hline$m=9$ & 0. 198 & o. $15^{8}$ & o. 14 I & o. 132 & o. 126 & o. I 23 & 0. I 208 & 0.119 & o. 1177 & o. I I66 & $\frac{1}{9}$ \\
\hline$m=\mathrm{II}$ & 0.2345 & 0.1583 & 0.1316 & 0.1185 & O. I I I I & o. 106 & 0.103 & o. 1009 & 0.0992 & 0.0979 & $\frac{1}{11}$ \\
\hline$m=13$ & 0.351 & 0.1755 & 0.1317 & 0.1124 & 0.102 & $0.96 \mathrm{I}$ & 0.0920 & 0.08916 & 0.08702 & 0.08533 & $\frac{1}{13}$ \\
\hline$m=15$ & 0.995 & 0.2214 & 0.1413 & o. 1126 & 0.0986 & 0.0903 & 0.08492 & 0.0813 & 0.07865 & 0.07665 & $\frac{1}{15}$ \\
\hline$m=17$ & 1.000 & 0.3444 & 0.1644 & 0.1185 & 0.0985 & 0.0875 & 0.0807 & $0.076 \mathrm{I}$ & 0.0729 & 0.0706 & $\frac{1}{17}$ \\
\hline$m=\mathrm{Ig}$ & 0.351 & I. 000 & 0.214 & o.132 & o. I0o & 0.0875 & 0.0788 & 0.073 & 0.069 & 0.0662 & $\frac{1}{19}$ \\
\hline
\end{tabular}

$$
\text { TABLE OF } \sin \frac{\pi}{2 N_{s p}} \div \sin \frac{m \pi}{2 N_{s p}}
$$

As $k_{r m}$ is in most cases much less than one, it appears that the harmonics are much more reduced by the belt-differential action than is the fundamental. 
In the case of a fractional pitch or short-chord winding, there is a phase difference between the E.M.F.'s in the two sides of each coil, or in the ordinary two layer winding, this same phase difference exists between the E.M.F.'s of the two belts or groups of coil sides, which together make up one circuit between taps. This introduces another reduction of the resultant E.M.F. If $\lambda_{c}$ is the coil pitch in terms of full pitch, the pitch differential factor between the two belts is, for the fundamental (see Fig. 29), $\overline{O B} \div(\overline{O A}+\overline{O B})=\sin \frac{\pi}{2} \lambda_{c}$, and for the $m$ th harmonic is $\sin \frac{m \pi}{2} \lambda_{c}$. The ratio of the latter to the former will be designated the pitch reduction factor for the $m$ th harmonic; it is

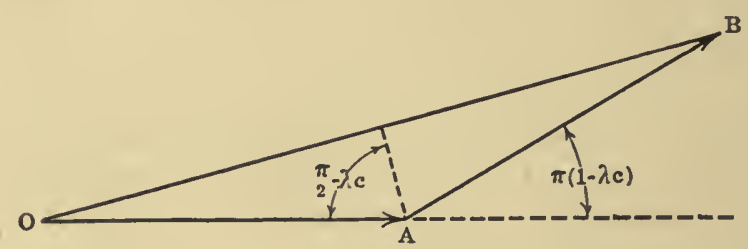

FIG. 29.

$k_{p m}=\frac{\sin \frac{m-\frac{\pi}{2} \lambda_{c}}{\sin \frac{\pi}{2} \lambda_{c}}}{\text { - per cent. of } m \text { th harmonic in full pitch winding }}$.

This is plotted in Fig. 30 for various pitches and harmonics (signs disregarded).

If the phase belt begins and ends in the middle or within a coil or slot bundle, there is a still farther reduction of the resultant E.M.F. The corresponding fractional slot reduction factor is

$$
k_{s m}=\left\{\begin{array}{l}
\cos \frac{m \pi}{2 N_{s p}} \text { (for half-slot connection). } \\
\cos \frac{m \pi}{2 N_{s p}} \div \cos \frac{m \pi}{6 N_{s p}} \text { (for a one-third or a two- } \\
\cos \frac{m \pi}{2 N_{s p}} \div \cos \frac{m \pi}{4 N_{s p}} \text { (for a one-fourth or a three- } \\
\text { fourths slot connection). }
\end{array}\right\} .
$$


The final ratio of the per cent $m$ th harmonic in the phase E.M.F. to the per cent $m$ th harmonic in the flux distribution curve is

$$
k_{m}=k_{r m} \times k_{p m} \times k_{s m}=\frac{\sin \frac{m \pi}{2} \lambda_{c}}{\sin \frac{\pi}{2} \lambda_{c}} \times \frac{\sin \frac{m \pi}{p^{\prime}}}{\sin \frac{\pi}{p^{\prime}}} \times \frac{\sin \frac{\pi}{2 N_{s p}}}{\sin \frac{m \pi}{2 N}-k_{s p}} .
$$

It remains to consider the phases of the harmonics in the resultant E.M.F.

The total phase rotation of the fundamental is $\frac{2 \pi}{p^{\prime}}$, and the phase

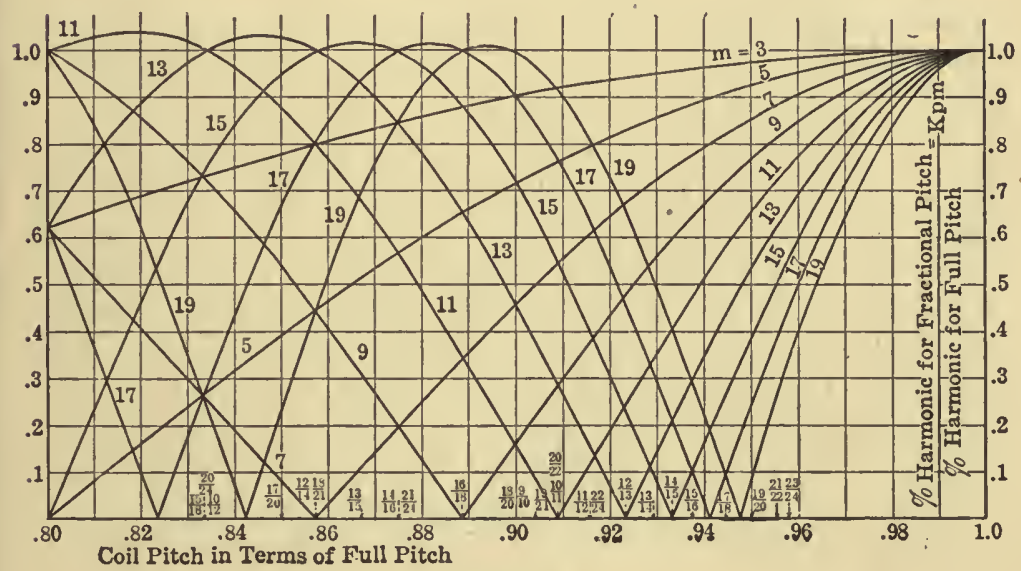

FIG. 30 .

of the fundamental of the resultant E.M.F. with respect to the fundamental of slot No. $\mathrm{I}$ is

$$
\alpha_{1}=\frac{\pi}{p^{\prime}}-\frac{\pi}{2 N_{s p}}
$$

The total phase rotation of the $m$ th harmonic is $\frac{2 m \pi}{p^{\prime}}$.

Let $w=$ nearest whole number less than $\frac{m}{p^{\prime}}$ and $w_{s}=$ nearest whole number less than $\frac{m}{2 N} N_{s p}$. Then the equivalent phase rotation of the 
$m$ th harmonic is $2 \pi\left(\frac{m}{p^{\prime}}-\omega\right)$, and the phase of the $m$ th harmonic of the resultant E.M.F. with respect to the $m$ th harmonic of slot No. I is, in $m$ th harmonic radians,

$$
\alpha_{m}=\pi\left(\frac{m}{p^{\prime}}-\omega\right)-\pi\left(\frac{m}{2 N_{s p}}-\omega_{s}\right) .
$$

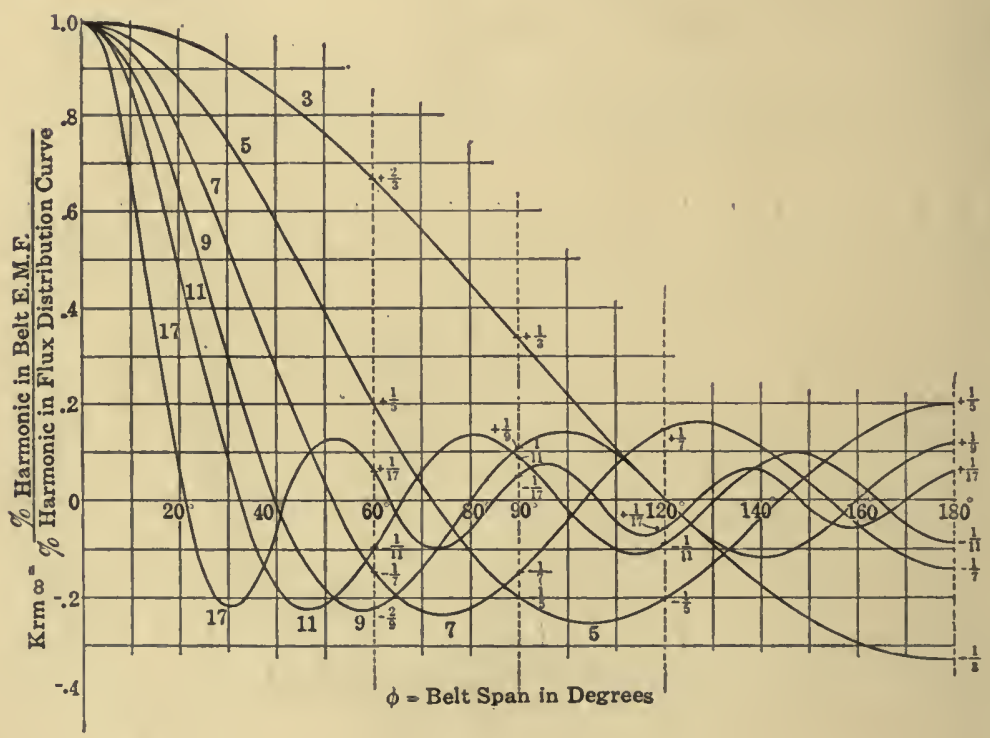

FIG. 3r.

Thus in passing from the slot E.M.F. to the resultant E.M.F., the relative phase of the $m$ th harmonic with respect to the fundamental is changed by an amount (in $m$ th harmonic radians),

$$
\beta_{m}=\alpha_{m}-m \alpha_{1}=-\pi\left(\omega-\omega_{s}\right) .
$$

$\beta$ is therefore always a multiple of $\pi$. Moreover, $\omega s$ is practically always zero. Therefore, $\beta_{m}$ is zero when $\omega$ is even and $\pi$ when $\omega$ is odd; i.e., the $m$ th harmonic can only be reversed and not otherwise changed in phase from the slot E.M.F. In order to see this relation more clearly, consider an infinite number of slots, and 
VOLTAGE RATIO IN SYNCHRONOUS CONVERTERS 231

designate the angle of belt span by $\phi$. The belt reduction factor is then

$$
k_{r m} \infty=\frac{I}{m} \sin \frac{m \phi}{2} \div \sin \frac{\phi}{2}, . . . \quad . \quad \text { ( . . ( }
$$

which is plotted in Fig. 3I for different values of the belt span ( $\phi)$ and for several harmonics. The reversal of the various harmonics with the change in belt span is here shown clearly.

\section{TAP Voltage}

For this purpose Eq. (I) may be written:

$e^{\prime}=a_{1}\left[q_{1} \sin \left(\omega t+\theta_{1}\right)+q_{3} \sin \left(3 \omega t+\theta_{3}\right)\right.$

$$
\left.+q_{5} \sin \left(5 \omega t+\theta_{5}\right)+\ldots q_{m} \sin \left(m \omega t+\theta_{m}\right)+\text { etc. }\right] \text {. }
$$

where

$$
q_{m}=\sqrt{q_{a m^{2}}+q_{b m^{2}}} \text { and } \theta_{m}=\tan ^{-1} \frac{b_{m}}{a_{m}} .
$$

The $m$ th harmonic in the tap E.M.F. is then

$$
k_{m} q_{m} \sin \left(m \omega t+\theta_{m}+\pi \omega\right) .
$$

The amplitude of the fundamental is

$$
a_{t 1}=k_{d 1} \frac{2 N}{p^{\prime}} q_{1} a_{1}
$$

where $k_{d 1}$ may be taken as equal to $\frac{p^{\prime}}{\pi} \sin \frac{\pi}{p^{\prime}}$ the limiting value of Eq. (5), as $N_{s p}$ approaches infinity. Then

$$
a_{t 1}=\frac{2}{\pi} N a_{1} q_{1} \sin \frac{\pi}{p^{\prime}}=q_{1} E_{1} \sin \frac{\pi}{p^{\prime \prime}}, \ldots . .
$$




\section{GENERAL DIAGRAMS FOR SYNCHRONOUS MOTORS}

and the complete tap voltage is

$$
\begin{aligned}
e_{t}=q_{1} E_{1} \sin \frac{\pi}{p^{\prime}}\left[\sin \omega t+k_{3} q_{3} \sin \left(3 \omega t+\theta_{3}+\pi \omega\right)\right. \\
\left.+k_{5} q_{5} \sin \left(5 \omega t+\theta_{5}+\pi \omega\right)+\text { etc. }\right] .
\end{aligned}
$$

The root mean square tap voltage is then

$$
E_{t}=\frac{q_{1} E_{1}}{\sqrt{2}} \sin \frac{\pi}{p^{\prime}} \sqrt{\mathrm{I}+k_{3}^{2} q_{3}^{2}+k_{5}^{2} q_{5}^{2}+k_{7}^{2} q_{7}^{2}+\text { etc. }} .
$$

and the ratio of brush to tap voltage is

$$
\left.K_{b t}=\frac{E_{d c}}{E_{t}}=\frac{\sqrt{2}}{q_{1} \sin \frac{\pi}{p^{\prime}}} \times \frac{\left(\mathrm{I}+\frac{1}{3} q_{a_{3}}+\frac{1}{5} q_{a 5}+\frac{1}{3} q_{a 7}+\right.\text { etc. ...) }}{\sqrt{\mathrm{I}+k_{3}^{2} q_{3}^{2}+\bar{k}_{5}^{2} q_{5}^{2}+k_{7}^{2} q_{7}^{2}+\text { etc. }}}, \quad \text { ( } 5\right)
$$

or

$$
K_{b t}=\frac{\sqrt{2}}{q_{1} \sin \frac{\pi}{p^{\prime}}} K_{q}
$$

when

$$
K_{q}=\frac{\left(\mathrm{I}+\frac{1}{3} q_{a_{3}}+\frac{1}{3} q_{a_{3}}+\frac{1}{5} q_{a_{5}}+\text { etc. }\right)}{\sqrt{\mathrm{I}+k_{3}^{2} q_{3}^{2}+k_{5}^{2} q_{5}^{2}+k_{7}^{2} q_{7}^{2}+\text { etc. }}} . \quad . . . .
$$

\section{Three-Part Pole}

With a normal flux distribution, or with a three-part pole symmetrical distortion, the $q_{b}$ 's are zero, $q_{1}=\mathrm{I}$ and $q_{m}=q_{a m}$. The denominator of $K_{q}$ is nearly unity, always a little greater. The numerator may differ considerably from unity on either side, according as the $q_{s}$ 's are positive or negative, slightly less than unity for undistorted flux distribution. Thus $K_{b t}$ will be slightly less than as given by the usual approximate formula. Any variation in the $q_{a}$ 's, due to a symmetrical distortion, causes a variation in the voltage ratio, but also in the harmonics of the tap E.M.F.'s.

The relation between these two changes is shown in Fig. 32 for the $180^{\circ}$ E.M.F., and for the third, seventh, eleventh, and seventeenth harmonics, each being taken separately. 
In order to change the relative voltage ratio from $I$ to .9 by third harmonic control, involves a ro per cent third harmonic in the diametral tap E.M.F., which corresponds to about 30 per cent third harmonic in flux distribution curve. To make the same change by seventeenth harmonic control, involves a I 2 per cent seventeenth

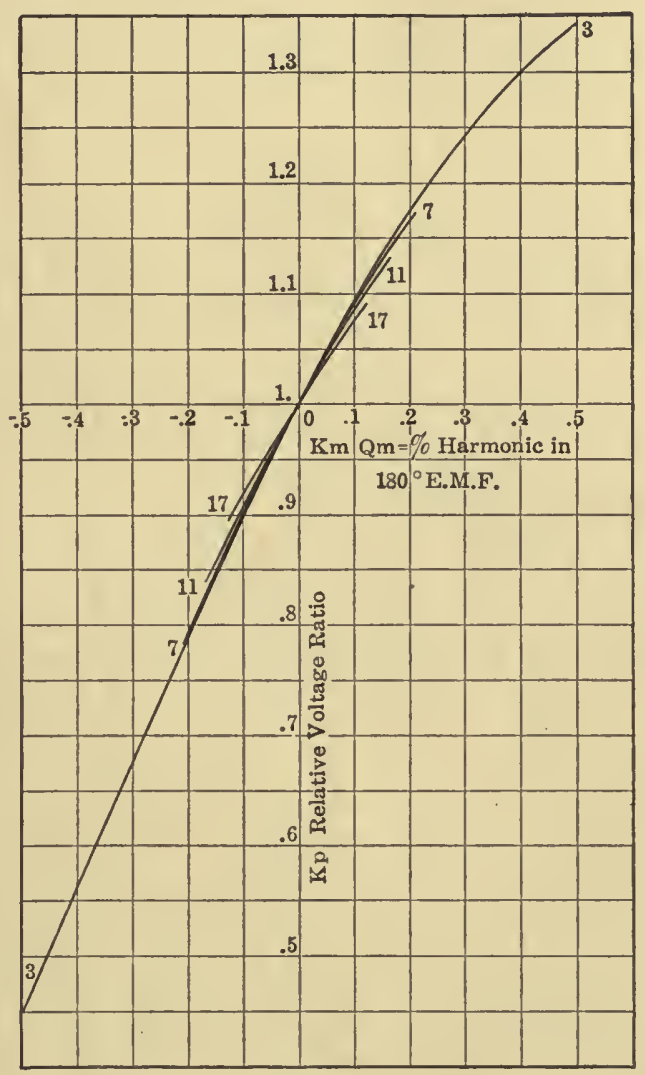

FIG. 32 .

harmonic in diametral tap E.M.F., and a seventeenth harmonic in the flux distribution curve of $\frac{\mathrm{I} 2}{k_{\tau 17}}$ per cent. For twenty slots per pole, this would mean a I 50 per cent seventeenth flux harmonic. A combination of harmonics produces about the same result as a single one of the same total per cent magnitude. 


\section{GENERAL DIAGRAMS FOR SYNCHRONOUS MOTORS}

The use of fractional pitch windings and fractional-slot connections, will reduce some of the harmonics; but as commutation limits the pitch reduction to a comparatively small range, only the higher harmonics are affected.

If the voltage ratio could be under control by a pure third harmonic in the flux distribution curve, the resulting third harmonic in the phase E.M.F. could be eliminated from the line voltage by connecting the transformer primaries in star.

\section{Two-Part PoLE}

This gives unsymmetrical distortion, and a lateral shifting of the flux distribution curve; i.e., the $q_{b}$ 's are no longer zero, particularly

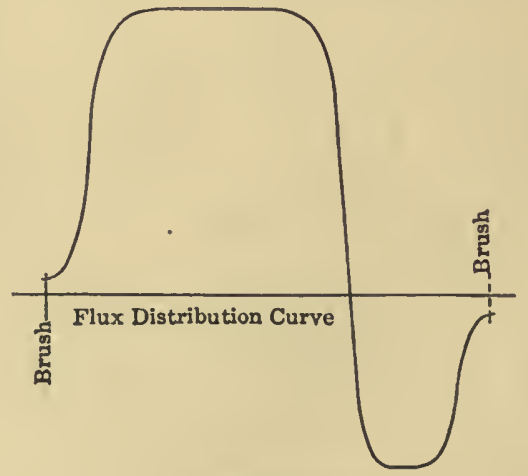

FIG. 33.

$q_{b 1}$. Thus $q_{1}=\sqrt{I+q_{b 1}^{2}}$ is no longer unity, and $K_{b} t$ is changed by $q_{1}$ as well as by $K_{q}$. If these changes are in the same direction, the harmonics in the tap E.M.F. will be obviously less for a given distortion.

In an actual machine for which the flux distribution curve is shown in Fig. 33, the voltage was

$$
\frac{E_{d c}}{E_{t_{180^{\circ}}}}=\frac{\sqrt{2}}{q_{\mathrm{I}}} \frac{\left(\mathrm{I}+\frac{1}{3} q_{a 3}+1 q_{b_{5}}+\text { etc. }\right)}{\sqrt{\mathrm{I}+k_{3}^{2} q_{3}^{2}+k_{5}^{2} q_{5}^{2}+\text { etc. }}}=\frac{\sqrt{2}}{\mathrm{I} .295} \frac{0.862}{\mathrm{I} .0 \mathrm{I} 6}=\sqrt{2} \times 0.655,
$$

less than two-thirds of the ratio for a sinusoidal flux distribution curve, and just about two-thirds of that for a normal undistorted 
field. The total harmonics were $2 \mathrm{I} .6$ per cent in the $180^{\circ}$ E.M.F., and only 7.2 per cent in the $120^{\circ}$ E.M.F., which are considerably less than is possible with a three-part pole. Moreover, it is quite possible that a more careful design of the pole faces might result in a farther reduction in the harmonics of the tap E.M.F.'s. Fractional pitch winding and fractional slot connections, would reduce them still slightly farther. 


\section{PART III}

METHODS OF CALCULATION OF THE ARMATURE REACTIONS (DIRECT AND TRANSVERSE) OF ALTERNATORS

\section{CHAPTER I}

\section{METHODS OF CALCULATION OF THE ARMATURE REACTIONS (DIRECT AND TRANSVERSE) OF ALTERNATORS ${ }^{1}$}

THE author here proposes to explain and complete the theory of "two armature reactions," which was enunciated by him several years ago, ${ }^{2}$ and which has recently been adopted, with slight modifications, by M. Rey, ${ }^{3}$ M. R. V. Picou, ${ }^{4}$ and M. Guilbert, ${ }^{5}$ in France; Professor Arnold ${ }^{6}$ in Germany; and Mr. Herdt ${ }^{7}$ and Messrs. Hobart and Punga ${ }^{8}$ in the United States. The notable authority of all these

${ }^{1}$ A paper by Prof. André Blondel, Ecole des Ponts et Chausées, presented before the International Electrical Congress at St. Louis, in 1904. Reprinted from the Transactions, Vol. I, pp. 635-668.

2 "On the empirical theory of alternators, "L'Industrie Electrique, Nov. ro and 25,1899 . This is the first publication in which the reaction in alternators was analyzed, and possesses undisputable priority over all those which are mentioned below on the subject of the two reactions.

${ }^{3}$ M. Rey. Rapports, International Congress of Electricians, Igoo.

'M. R. V. Picou. Bulletin de la Sociêté Internationale des Electriciens July, rgo2.

${ }^{8} \mathrm{C}$. F. Guilbert. Eclairage Electrique, March 7 and $\mathrm{I}_{4}$ and April, $\mathrm{IgO}$, and La Révue Technique, June, Igo3.

- E. Arnold. Elek. Zeit., 1902, page 250. Arnold, as pointed out farther on, has reduced the generality of the method, in contradistinction to the other authors mentioned.

${ }^{7}$ L. A. Herdt. Trans. Amer. Inst. El. Eng., May, 1902, and Eclairage Electrique, February $\mathrm{r}_{4}, 1903$.

${ }^{8}$ Hobart and Punga. Trans. Amer. Inst. El. Eng., A pril 22, Ig04. 
authors in the matter of dynamo-machine construction has made me read their communications with great interest, and as I have observed that in certain cases my own view has not been well understood, I consider it desirable to present certain supplementary considerations to make this theory still more simple and to complete it finally. At the outset it should be pointed out that my diagram should not be considered as belonging to the category of E.M.F. but rather to that of ampere-turn diagrams. The two classes are often equivalent, because if one commences with E.M.F.'s one proceeds with fluxes, and ends necessarily with ampere-turns. But I desire to reduce to a minimum the complication of considerations relative to the saturation of fieldmagnets, of which I fear the difficulties have been needlessly exaggerated.

In what follows I will refer first, very briefly, to the essential points of my method of $\mathrm{I} 899$, and I will show in what points it has been improved, or is susceptible of improvement.

\section{Part I. Diagram of Operation.}

Principles of the Theory of Two Reactions. I have long been surprised that polyphase alternators and direct-current machines have not been treated from the point of view of reaction, since these phenomena are fundamentally of absolutely the same order, since the dephasing alternating current produces effects of the same order as the displacing of the brushes in a direct-current dynamo. It is known that in the latter case the displacement causes a direct magnetic reaction to be developed, whilst in the neutral position there is only a transverse reaction. By similar reasoning as to the automatic dephasing of alternating currents and of the property which polyphase currents possess of being decomposable into active and reactive components, I have been led to the following proposition:

When an alternator supplies a current dephased by an angle $\psi$ with respect to the internal induced E.M.F., the armature reaction may be considered as the resultant of a direct reaction produced by the reactive current $\sin \psi$ and a transverse reaction due to the active current I $\cos \psi$.

In addition to the above, the stray magnetic fields must be taken into account. These are proportionate to the currents and in phase with them. We will consider them later on.

The second fundamental proposition of this theory is the following:

The two reactions (direct and transverse) and the stray flux take 
place in three different magnetic paths; only the direct reaction acts in the main circuit of the field magnets, while the transverse reaction and the stray fields act, in general, upon circuits of low magnetic density.

The conclusion which I have drawn from the above is that, in general, the direct reaction should be expressed as a counter M.M.F.; that is to say, by a number of ampere-turns equivalent to the demagnetizing effect of the armature. ${ }^{1}$ The total armature M.M.F. per complete magnetic circuit or per pair of poles is

$$
\frac{K N}{2} I \sqrt{2},
$$

where $K$ is a coefficient of reduction and $N$ the armature conductors per pair of poles. I have formally given for asynchronous motors a practical value which is approximately the same for alternators, viz.:

$$
K=\left(\frac{2}{\pi}\right)^{2} k \text {, }
$$

$k$ being the coefficient of reduction which appears in the formula of E.M.F. written under the form

$$
E=\frac{k \omega N \phi \times 10^{-8}}{2 \sqrt{2}} .
$$

Here $N$ is the number of peripheral conductors for one phase and $\omega$ the velocity of pulsation. It is the direct reaction which produces, almost entirely, the variation of terminal voltage. As to the transverse reaction and the reaction of stray fields, with the assumption that the armature is unsaturated, as I assumed and as M. Guilbert also assumes, they may be expressed simply by the coefficients of selfinduction $l$ and $s$.

More recently $\mathrm{I}$ have indicated ${ }^{2}$ that the transverse reaction could also easily be expressed in ampere-turns.

The analysis of the phenomena taking place in the alternator leads therefore to a new proposition, formulated in my articles of 1899 .

1 No notice is here taken of one of the cases considered by the author in 1899 ; namely, that in which all the machine is well below saturation, because it is only susceptible of very rare applications; moreover, it has been treated with more detail by M. Jean Rey in a very interesting communication presented to the Congress of 1900 , in which the reader will find an interesting example of a calculation of reactive coefficients in a machine actually built by this method, which has since been followed by various authors.

2 "Theory of Synchronous Motors," Vol. I. Paris, Gauthiers-Villars, 1900. 
The dephasing $\psi$ of the current is regulated entirely by the numerical value of the transverse reaction, which, on the contrary, has little effect upon the E.M.F.

This proposition has been demonstrated in the case of unsaturated armatures, as I have just stated, but it is general and remains in effect even in the case where the circuit of the transverse reaction approaches saturation. The demonstration of this will be given below.

Diagram of E.M.F.'s and Currents of an Alternator with Unsaturated Armature and with Saturated Field Magnet. The diagram in Fig. ${ }^{1}$ reproduces Fig. 5 of my first paper, supplemented by the

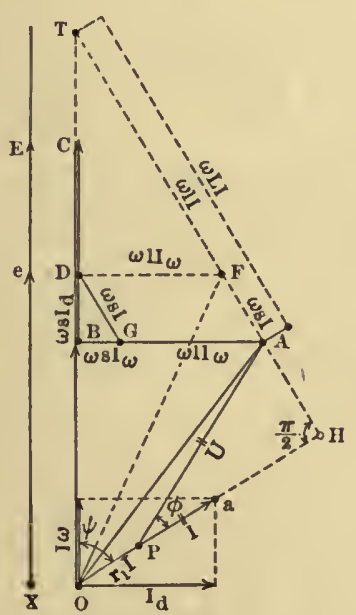

FIG. I.

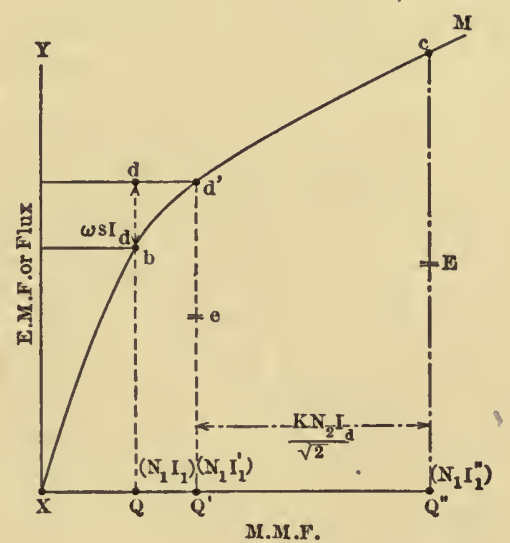

FIG. 2.

definitions of Fig. 2. $r^{\prime}$ represents the apparent resistance, that is to say, the ohmic resistance augmented by the effects due to Foucault currents; $\phi$ is the difference of phase in the external circuit, and $\psi$ is the difference of phase with respect to the internal E.M.F. It is proposed to calculate the excitation necessary to develop an E.M.F. $U$ at the terminals under a current-delivery $I$ dephased by $\phi$. We have $O P=r^{\prime} I$ and $P A=U$, with the angle $A P a=\phi$.

Let $O T$ be the direction, as yet unknown, of the internal E.M.F. $e$; the perpendicular $A B$ let fall from $A$ upon $O T$ is the sum of the transverse reaction:

$$
A G=\omega l I \cos \psi,
$$

1 The subscript $w$ indicates the active or energy component and the subscript $d$ the reactivc or quadrature reactive component. 
(where $l$ is the transverse self-inductance and $\omega(=2 \pi f)$ the speed of pulsation); and of a part of the stray field reaction-

$$
G B=\omega s I \cos \varphi^{\prime 1}=\omega s I_{\imath}
$$

where $s$ is the self-induction of the stray fields.

The segment GD perpendicular to $I$ represents the E.M.F. of reaction of the stray fields, $\omega s I$, and the segment

$$
B D=\omega s I \sin \psi=\omega s I_{d}
$$

represents the second component of the stray-field reaction; thus in $O D$ is obtained the value of the effective E.M.F. $e$, which should be obtained by the resultant excitation.

The value of the angle $\psi$ is determined by expressing simply the relations between the elements of the figure. Let us analyze the broken line $O P A B$ into components upon $O B$, and $B .4$; whence

and

$$
\begin{aligned}
e & =r^{\prime} I \cos \psi+U l \sin (\psi-\phi) \\
\omega(l+s) I \sin \psi & =r^{\prime} I \sin \psi+U \sin (\psi-\phi)
\end{aligned}
$$

$$
\operatorname{tg} \psi=\frac{U \sin \phi+\omega(l+s) I}{U \cos \phi+r^{\prime} I} .
$$

The angle of real dephasing $\psi$ is thus determined solely by a knowledge of the transverse reaction. This equation, which was given by the author in 1899, is evidently equivalent to the following construction.

From the point $A$ a perpendicular $A H$ is drawn to the direction of the current $I$, and a segment $A F$ is drawn upon this line equal to $\omega s I$; then a segment $F T=\omega l I$; finally the point $O$ is joined to the point $T$, and thus is obtained the angle $\psi$ and the position of the required vector $O D$ representing the total effective E.M.F. e. To determine the necessary ampere-turns for the production of this E.M.F., it is only necessary to employ the open circuit characteristic or saturation curve of the alternator.

1 The segment $B T$ intercepted by $O T$ will evidently be equal to

$$
B D+D T=\omega I I \sin \psi+\omega s I \sin \psi .
$$


Diagram of Ampere-Turns in the Case of Unsaturated Armature. The consideration of ampere-turns does not need to appear in the method, as is evident in the case of an unsaturated armature, until after having traced the diagram of E.M.F.'s. The excitation ampereturns are drawn, if desired, along the direction of the vector $O C$, in order to facilitate certain comparisons; but the calculation of ampereturns is no longer in this method a vectorial, geometric calculation, but a scalar calculation, and may be made upon the saturation curve as calculated or observed, which represents the induced E.M.F. (or the useful flux traversing the armature) as a function of the total ampereturns applied on the field magnets.

Their determination is based on the following facts:

(I) The reactive counter-ampere-turns of the armature are proportional to the number of peripheral conductors on the armature per pair of poles, $N_{2}$, which supply a number of turns equal to $\frac{N_{2}}{2}$; but these turns do not act in unison, partly because they belong to different phases, and partly because they are not in the same slots. For this reason it is necessary to apply a reduction-coefficient $K$. The effective reactive current $I_{2} \sin \psi$ thus gives rise to an M.M.F. exactly opposite to that of the field magnets, and having for its magnitude in counter-ampere-turns

$$
C A T=\frac{K N_{2} I_{2} \sin \psi \sqrt{2}}{2}=\frac{K N_{2} I_{d}}{\sqrt{2}} .
$$

(2) These counter ampere-turns act, on the one hand, upon the circuit common to the field magnets and the armature; and on the other hand, upon the circuit of the armature and of the stray magnetic fields. This may be represented diagrammatically as in Fig. 3, in which the full lines $A B C D E F$ represent the principal magnetic circuit with its two M.M.F.'s acting in the opposite directions $N_{1} I_{1}$ and $\frac{K N_{2} I_{d}}{\sqrt{2}}$, and the dotted lines $A D A D$ indicate the stray circuits in which the lines of force escape, either between the teeth of the armature $f_{2}$ or between the polar horns $t_{1}$. In reality the stray fields

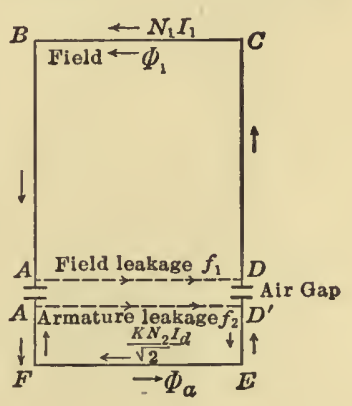

FIG. 3 . $f_{1}$ of the field magnet are not concentrated along any single path, but 
are spread out more or less over the entire length of the principal circuit up to its entrance into the armature turns. This fact is, however, unimportant, as has recently been shown by M. Guilbert (see Eclairage Electrique, December, 1903).

(3) The self-inductance of the armature is produced by the stray fields $f_{2}$, supposedly attributed to the effect of the armature. If we call $R_{f_{2}}$ the reluctance of the circuit of the stray fields $f_{2}$, and $R_{a}$ the reluctance of the armature, the stray field produced by the armature across itself, is expressed in practical units $\left(N_{2}\right.$ being the number of peripheral conductors per pair of poles),

$$
f_{2}=0.4 \pi\left\{\frac{K N_{2} I_{2} \sqrt{2} \sin \psi}{2\left(R_{a}+R_{f_{2}}\right)}\right\}=\frac{0.2 \pi K N_{2} I_{2} \sqrt{2} \sin \psi}{R_{f_{2}}},
$$

assuming that we can neglect $R_{a}$ with respect to $R_{f_{2}}$, and thus produce an E.M.F.

$$
E_{f 2}=\frac{k N_{2} \omega f_{2}}{2 \sqrt{2}}=\frac{k N_{2} \omega \times 0.1 \pi K N_{2} I_{d}}{R_{f_{2}}}
$$

It results from this that the E.M.F. of self-induction that we have called $\omega s I_{d}$ can be considered as produced simply by a stray field $f_{2}$, which is added to the stray field of the field magnets $f_{1}$.

Upon the saturation curve $X M$ (Fig. 2) defined as above, the point $b$ which corresponds to the E.M.F. $O B$ (Fig. I), represents the $N I$ necessary to force the useful flux througl the field magnets into the armature. Adding to the flux $\Phi_{a}$ the stray field of the armature $f_{2}$, there is obtained the virtual E.M.F. $O D=e$, corresponding to the total flux emanating from the poles into the air-gap; the corresponding abscissa $X Q^{\prime}$ represents the necessary field-winding ampereturns $N_{1} I_{1}{ }^{\prime}$, without taking into account the increase $\Delta f_{1}$ of the stray field $f_{1}$ of the field magnet.

(4) The stray field of the field magnets $f_{1}$ is inversely proportional to the reluctance of the stray path $R_{f_{1}}$ between the poles and directly to the difference of magnetic potential between the poles. This latter is formed of two parts; one part is the drop of magnetic potential necessary to force the flux through the armature and air-gap, the other part, the reactive counter-ampere-turns of the armature calculated as above.

(5) Every increase in the ampere-turns of the field magnet increases 
the stray flux $f_{1}$ of the field magnet, in a manner sensibly proportional to the increase of the ampere-turns of the field. If, therefore, the field-magnet ampere-turns are increased by

$$
K N_{2} I_{d} \frac{\sqrt{2}}{2}
$$

in order to compensate for the counter-ampere-turns, $C A T$, of the armature, the stray flux $f_{1}$ produced by the field magnet following the circuit $B A D C$ would be increased by a quantity,

$$
\Delta f_{1}=\frac{0.2 \pi K N_{2} I_{d} \sqrt{2}}{R_{1}+R_{f_{1}}}=\frac{0.2 \pi K N_{2} I_{a} \sqrt{2}}{R_{f_{1}}} \text { approximately }=0.4 \pi \frac{(C A T)}{R_{f_{1}}},
$$

$R_{1}$ being the reluctance of the field magnet.

This increase of the flux through the field magnet increases the magnetic density in the latter and demands consequently a correction, as I pointed out in 1899 , without tracing it in detail. At that time I conducted the inquiry simply as follows, supposing the rôle of the field magnets to be sufficiently unimportant to permit approximate correction being applied.

Let $B_{1}$ be the flux density in the field magnet, corresponding to the no-load E.M.F. $e$, that is to say, to the flux $\phi_{a}+f_{1}$; and let us call $v_{1}$ the Hopkinson coefficient $\frac{\phi_{a}+f_{1}}{\phi_{a}}$. The full-load induction will be

$$
\begin{aligned}
B_{1}^{\prime}=B_{1} \frac{\phi_{a}+f_{1}+\Delta f_{1}}{\phi_{a}+f_{1}} & =B_{1}\left\{\mathrm{I}+\frac{0.4 \pi(C A T)}{R_{f 1}\left(\phi_{a}+f_{1}\right)}\right\} \text { approximately } \\
& =B_{1}\left\{\mathrm{I}+\frac{(C A T) f_{1}}{N_{1} I_{1}\left(\phi_{a}+f_{1}\right)}\right\} \\
& =B_{1}\left\{\mathrm{I}+\frac{(C A T)}{N_{1} I_{1}} \cdot \frac{v_{1}-\mathrm{I}}{v_{1}}\right\}
\end{aligned}
$$

and consequently the total ampere-turns will be increased by the quantity by which the change from $B_{1}$ to $B_{1}{ }^{\prime}$ increases the ampereturns (which we shall call $N_{1} I_{1}$ ) specially absorbed by the reluctance of the field magnets in the condition considered. The point $d^{\prime}$ upon the curve will be in consequence displaced toward the left by the quantity corresponding to this increase of ampere-turns.

Starting from the point $Q^{\prime}$ duly corrected, it is sufficient to take a length representing the ampere-turns equal to $C A T$ of the armature 
in order to obtain the total necessary ampere-turns $O Q^{\prime \prime}$. Fig. 4 shows how the diagrams of E.M.F.'s and of ampere-turns may be united upon a single sheet.

The preceding reasoning may be summed up in the following simple equations, employing ordinary language:

The fall of magnetic potential in the armature and air gap is a function of the flux utilized in the armature $\phi_{a}+$ the armature stray flux $f_{2}$.

The magnetic difference of potential between the pole pieces $=$ the fall of magnetic potential in the armature and air-gap + the reactive counter ampere-turns of the armature.

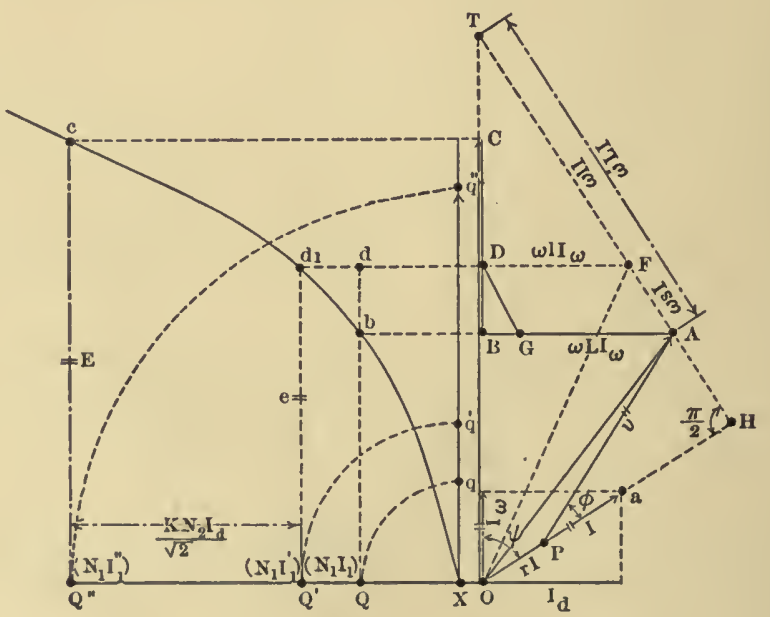

FIG. 4.

The magnetic stray field between the magnets $f_{1}+\Delta f_{1}=\mathrm{a}$ function of the difference of potential of the pole pieces.

The total stray magnetic fluxes=the fluxes $f_{1}+\Delta f_{1}$ the stray fields $f_{2}=f_{1}\left[\mathrm{I}+\frac{(C A T)}{N_{1} I_{1}} \frac{v_{1}-\mathbf{I}}{v_{1}}\right]+f_{2}$.

The total flux in the field magnet $=$ the useful flux +the flux of the total stray field $=\phi_{a}+f_{1}\left[\mathrm{I}+\frac{(C A T)}{N_{1} I_{1}} \cdot \frac{v_{1}-\mathrm{I}}{v_{1}}\right]+f_{2}$.

The total ampere-turns of the field magnet=the difference of magnetic potential between the pole pieces + the total drop of potential in the field magnet corresponding to the total flux $=$ the fall of potential 
in the armature +the back ampere-turns of the armature + the fall of potential in the air-gap+the fall of potential in the field magnets corresponding to the total flux.

When the alternator is unsaturated or but slightly saturated, this latter fall of potential corresponding to the total flux may be admitted proportional to the flux $\phi_{a}+f_{1}+f_{2}+\Delta f_{1}$

$$
=\left(\phi_{a}+f_{1}\right)+0.4 \pi(\mathrm{CAT})\left(\frac{\mathrm{I}}{R_{f_{1}}}+\frac{\mathrm{I}}{R_{f_{2}}}\right) .
$$

Thus the flux $\Delta f_{1}$ plays a part entirely similar to the flux $f_{2}$, and it may therefore be united with the latter in the coefficient of selfinduction of the armature. It must, however, be remarked that the flux $\Delta f_{1}$ only follows the magnetic circuit to the point of emergence of the flux from the field magnets, and only absorbs consequently the fraction $\frac{R_{1}}{R_{\text {total }}}$ of the ampere-turns which would be necessary to make it traverse the entire magnetic circuit. The virtual selfinduction $s$ which may be advantageously assumed will then have an approximate expression

$$
s=0.1 \pi K k N_{2}^{2}\left(\frac{\mathrm{I}}{R_{f_{3}}}+\frac{R_{1}}{R_{\text {total }}} \frac{\mathrm{I}}{R_{f_{1}}}\right),
$$

and this should be employed in the determination of the segment $B D$, as above.

In order to get the total necessary field-magnet ampere-turns $N_{1} I_{1}{ }^{\prime \prime}$, it is no longer necessary to add any excitation upon the field magnets except the ampere-turns neutralizing those of the armature $\frac{K N_{2} I_{d} \sqrt{2}}{2}$. The total ampere-turns $X Q^{\prime \prime}$ are thus obtained. If the armature current were suddenly suppressed, an E.M.F. $E=Q^{\prime \prime} c$ would appear in the armature on open circuit, which is that appearing with the same notation in Fig. 2.

The same construction may serve reciprocally to calcuate the fall of potential produced in an alternator having the excitation $X Q^{\prime \prime}$ for the reactive or wattless current $I_{d}$ in the armature.

Remark No. I, Upon the Case of an Unsaturated Armature. When the armature and the pole pieces are not saturated, the diagram of E.M.F.'s (Fig. I) is also a diagram of the flux, to a different scale, if care be taken to divide the values of the E.M.F.'s by the coefficient $\frac{k \omega N_{2}}{2 \sqrt{2}}$. 
Similarly, having given the magnetic reluctances sensibly constant for the direct flux of the armature and for the transverse flux (excluding the field magnet core), the same diagram may also present to a suitable scale the M.M.F.'s proportional to the flux, multiplied by the reluctance of the armature, of the air-gap, and of the pole pieces respectively. In that case, $O A$ represents the necessary ampereturns to force the flux through the said reluctances. $O B$ is the part of this M.M.F. furnished by the field magnets; $A B$ the part furnished

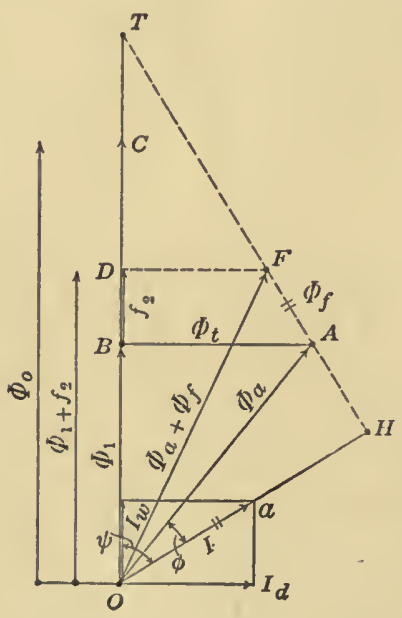

FIG. 5.

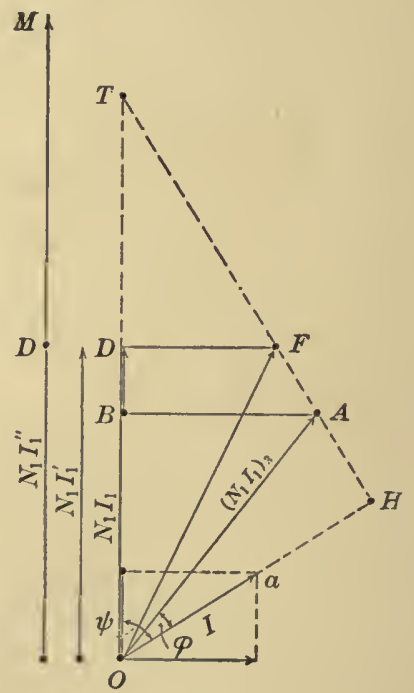

FIG. 6.

by the armature; $B D$ the supplement necessitated by the stray fields of the armature. ${ }^{1} D F$ represents the ampere-turns of distortion

$$
D F=\text { function of } \frac{K_{t} N_{2} I_{w}}{\sqrt{2}} \text {. }
$$

Calling $K_{\ell}$ a coefficient of distortion, or of transverse reaction, analogous to the coefficient $K$ of the direct reaction, and $f$, (the function)

${ }^{1}$ And eventually by the supplementary stray fields $\Delta \mathrm{f}_{1}$ in the particular case indicated above, where the effect of the stray field from the field magnets is referred to a supplementary term of the armature stray fields. 
the relation which connects the ampere-turns to be produced by the field-magnet with those utilized in the armature, we have similarly

$$
F T=\text { function of } \frac{K_{t} N_{2} I}{\sqrt{2}} \text {. }
$$

The total ampere-turns necessary to the emergence of the flux from the field magnets will be determined as above (Fig. 4): let $X Q^{\prime \prime}$ represent the magnitude equal to $N_{1} I_{1}{ }^{\prime \prime}$, to the scale of the new figure; the distance $O Q$ will evidently represent the ampere-turns absorbed by the field alone; it is this length $O Q$ which would in general be corrected by taking account of the stray field $\Delta f_{1}$. The method of correcting the stray fields indicated above may be employed; we will give further on another more nearly accurate-already suggested, moreover, by MM. Picou and Guilbert. ${ }^{1}$

Remark No. 2, upon the Subject of Diagram No. I. It is to be observed that following the respective values of the reactive coefficients, both direct and transverse, the point $C$, the extremity of the ąvailable E.M.F. on open circuit, may be either above or below $T$.

With an alternator of unsaturated field magnets, the two reactions have coefficients nearly equal, and $C$ may then coincide with $T$, when they are equal. Ordinarily, the coefficient of distortion, $K_{\ell}$, tends to be reduced, as we shall see, by a reduction in the breadth of the poles, while the coefficient $K$ has the opposite tendency. It results from this that $C$ tends to be above $T$. But the saturation of the field magnets lowers it the more as the saturation is greater; because this latter augments but slightly the supplementary ampere-turns necessary to compensate for those of the armature, but greatly diminishes the variation of the voltage between open circuit and full load. $C$ is, therefore, in general below $T$, as represented in the diagrams.

The same condition may be found even with saturation, with certain types of alternators, such for example as that which was exhibited in 1900 , in Paris, by the firm Sautter-Harlé. This machine, developed along a plan, formerly patented by Professor E. Thomson, of an iron rotor (inductor alternator), has a single armature, two exciting field-windings, and a yoke closing the magnetic circuit through

${ }^{1}$ The diagram of the present figure is analogous to a diagram recently published by M. E. Guilbert (loc. cit.); it differs, however, in that the line $F E$ is expressed as a function of the coefficient $K_{\ell}$ instead of the coefficient $K$, and that the expression of dephasing $\psi$ is thus presented as a function of the ampereturns. 
the shaft of the field magnet. It presents a supplementary air-gap of considerable reluctance around the shaft, and this air-gap is traversed only by the direct reaction. The coefficient of direct reaction is therefore rendered smaller than that of the transverse reaction, and if the supplementary stray fields $\Delta f_{1}$, analyzed above, are not exaggerated, $C$ will remain below $T$.

Those theoretical diagrams are not, therefore, liable to criticism which show $C$ below $T$. To propose placing $C$ always on $T$, as

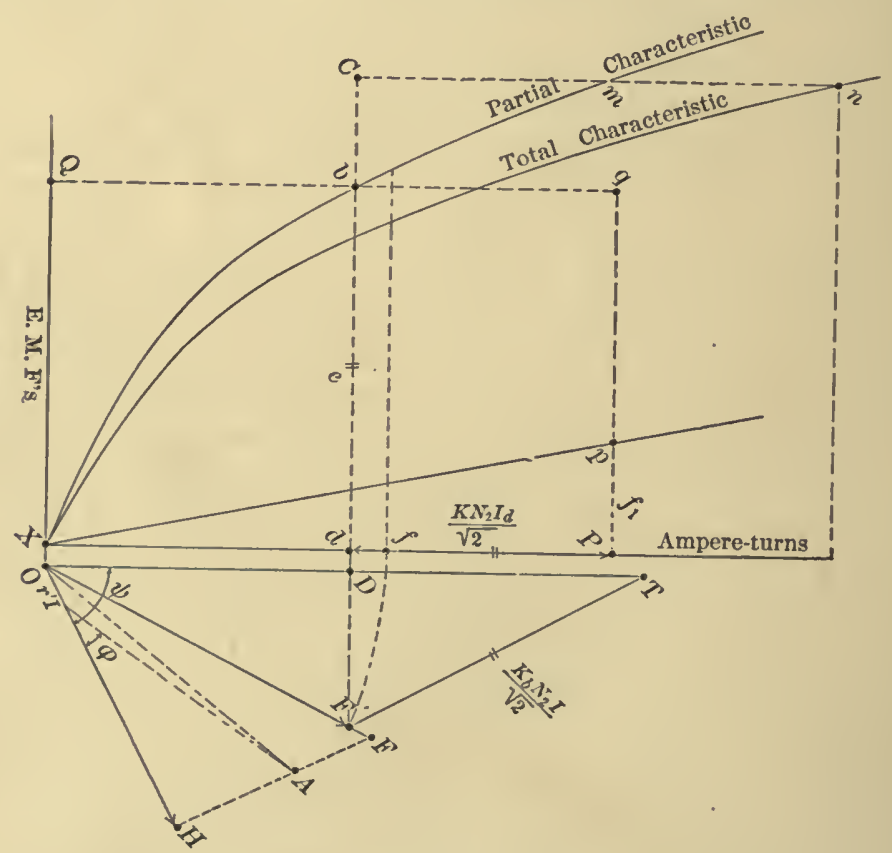

FIG. 7.

Professor Arnold has done, ${ }^{1}$ is contrary to the purpose of this method, namely, the calculation of the effects of saturation and of distortion according to rational principles.

The Case of a Saturated Armature. (Fig. 7.) The theory of two reactions permits also of treating the case of a saturated armature, by employing with the total characteristic, the characteristic of the armature alone (comprising the armature, air-gap, and pole pieces),

${ }^{1}$ E. Arnold, Elek. Zeit., I902, page 250. 
already employed moreover by MM. Bauch, Potier, Guilbert, and Picou.

For greater clearness of explanation I shall represent the characteristics upon the same diagram, but, in practice, they would be drawn upon separate figures. The curves are referred to the E.M.F's. as common abscissæ, and the ordinates of the two curves represent respectively the ampere-turns for the passage of a given flux (corresponding to the E.M.F.) in the magnetic circuit, with or without the field magnets. The difference of the ordinates equals then the ampere-turns absorbed by the field magnet alone, in the absence of stray magnetic fields.

This assumed, we shall then construct, according to custom ,the diagram of E.M.F.'s by adding to the voltage $U$ at the terminals the internal drop $r^{\prime} I$, then the loss by stray magnetic fields $A F$. We thus obtain the E.M.F. $O F$ produced in the armature. We find upon the characteristic of the armature the ampere-turns $O F^{\prime}$ corresponding to this E.M.F., and we lay them off on $O F^{\prime}$ in the direction of $O F$.

We may then observe that the distortion may be determined by the comparison of the ampere-turns of distortion with the useful ampere-turns in that part of the machine which does not include the field-magnets; because, in the transverse reaction, the reluctance of the pole pieces may be neglected in relation to that of the entrefer and of the armature (especially that of the teeth), and consequently attribute to the path of this reaction the same reluctance as in the path of the useful flux outside the field magnets. The determination of the angle $\psi$ (Fig. I) will then be transformed into simply replacing the self-inductance by the M.M.F.'s applied to the armature. The ampereturns $F^{\prime} T=\frac{K_{t} N_{2} I}{\sqrt{2}}$ will be laid off in the direction perpendicular to $I$, and $O T$ is joined; then from $F^{\prime}$ the perpendicular $F^{\prime} D$ is dropped, which will be equal to the ampere-turns of distortion

$$
D F^{1}=\frac{K_{t} N_{2} I_{v}}{\sqrt{2}}
$$

The line $O D$ will then represent the ampere-turns to be supplied by the field-magnets at the point of emergence, the remainder $D F^{\prime}$ being furnished by the armature itself.

It remains to determine the total field-magnet ampere-turns corresponding thereto, taking into account-the reluctance of the stray flux 
of the field magnets, properly so called. For this purpose it suffices to seek upon the characteristic of the armature the corresponding E.M.F. $d b=e$; the ordinate $Q b$ corresponding to this abscissa $e$ upon the curve of the armature measures the necessary ampere-turns to be produced between the poles, the necessary stray-fields $f_{2}$ included. We add thereto the ampere-turns equilibrating the direct reaction of the armature, that is to say,

$$
b q=\frac{K N_{2} I_{d}}{\sqrt{2}}
$$

The ordinate $Q q$ represents the difference of potential (subject to the factor $0.4 \pi$ ) necessary between the poles of the field magnet. From this may be deduced the value of the stray field $f_{1}$ between the pole pieces, which may be presented, for example, as a function of the magnetic difference of potential along the curve $X P$, which is sensibly a straight line; $P p$ will then represent the stray field $f_{1}$.

If, starting from $b$, a segment $b C$ be drawn representing $P p$ (measured to the same scale as the flux $d b$ corresponding to the E.M.F. $e$ ), and through $C$ we draw the straight line $C m$ parallel to $O P$, the latter will contain between the two characteristics a segment $m n$ which will represent the fall of magnetic potential in the field magnets under the influence of the total flux $d C$. The total necessary M.M.F. will thus be equal to $O P+m n$.

The diagram is thus established, taking into account the stray field both of the field magnet and of the armature. It is distinguished from those of Potier, ${ }^{1}$ Rothert, ${ }^{2}$ and Bauch, ${ }^{3}$ because it takes into account the transverse reaction with its real value; it takes account of the difference between the two coefficients of reaction $K$ and $K_{t}$ and is thus distinguished from the diagram of M. Guilbert ${ }^{4}$ for unsaturated field magnets; it finally differs in diagrammatic construction from the very ingenious diagram of the same author for saturated field magnets in the fact that it does not separate the air-gap from the armature, and is also much more simple.

Summing up, the employment of the diagram in Fig. 4 is to be recommended for the case in which there is no appreciable saturation either in the armature or in the field. In all other cases it seems

1 Potier, Eclairage Electrique, July 26, 1902.

2 A. Rothert, Elek. Zeit., 1899.

3 Bauch, Elek. Zeit., rgoz.

4. Muilbert, Revue Technique, April and May, 1904. 
preferable to employ the diagram Fig. 7, which lends itself better to determining the different elements without complications.

If it is desired to solve the inverse problem, that is, to determine the fall of potential under constant excitation as a function of the load, the preceding diagrams do not give a direct solution, but it is easy to employ them for an indirect solution, particularly in assuming constant the external dephasing $\phi$, and taking successively different values of the reactive current; for each value of $I_{d}$ the preceding construction will be followed in the opposite direction, and thus will be obtained the voltage at the terminals, the values of $\psi$ and of $I_{w}$. Thus may be traced a curve of voltage $u$ as a function of $I_{d}$, and of $I$ which is obtained therefrom. It is only necessary to seek upon this curve the point corresponding to the conditions required and the dephasing angle.

The problem is solved no longer for a single point, but along a complete curve, which is also comparatively easy.

Local Corrections of the Air-gap Due to Saturation (Second Approximation). The diagram Fig. 7 is established by supposing that the reactions act en bloc, and are represented by coefficients. The same is true of the diagram Fig. I. But if it be desired to follow the reality somewhat closer, it is well, once the diagram is determined by the aid of the coefficient $K$ (the calculation of which is explained later), to calculate upon the drawing the flux-density of the resulting field at each point along the air-gap, by the aid of magnetic potential curves (to be explained later) and of the local reluctance. In particular, if the teeth of the armature are saturated, they develop marked variations of the reluctance per unit of surface along the air-yap, and the flux calculated according to a mean value of reluctance may be sensibly modified thereby. This is the case not only for the transverse reaction, as has already been remarked by certain authors, but also for the direct reaction, which should not be set aside in this correction. The effect of this latter is to reduce the resultant flux. The curve of the diagram is, in fact, a solution of the first approximation necessary in order to determine the dephasing of the values of the active currents from the reactive currents. After these values have been obtained, a second approximation may be arrived at by tracing the flux-densities from point to point for determining the real flux. In general, however, the precision of the calculations is not sufficiently great to proceed upon this correction unless ample time may be afforded for the study. 
Case of Field Magnets with Divided Windings. In certain machines, particularly turbo-alternators, circular field magnets are found in which the windings are carried along the air-gap in slots like those of the armature. The preceding diagrams (Figs. I to 7) apply likewise to these machines only on the condition of assuming the two coefficients of reaction equal even when the field magnet is entirely divided into slots. Moreover, the field-magnet winding must be affected by a coefficient of ampere-turns $K_{1}$, reducing them to $K_{1} N_{1} I_{1}$ (with $K_{1}=0.4$ to 0.5 in completely uniform windings), and by the Hopkinson coefficient $v_{1}$, which is calculated like the stray field of a slot in an asynchonous motor. All the other coefficients are calculated as in the ordinary case, by supposing the breadth of the reactive flux equal to that of the field-magnet poles. This method has given me satisfactory results in practice for this type of machines.

\section{Part II. Calculation of Constants.}

Practical Calculation of Reactions. In order to apply the diagrams, the coefficients $s, l, K_{\ell}$ and $K$ must be determined ( $l$ and $K_{\ell}$ are of course only two expressions of one and the same coefficient). The stray coefficient is determined by known methods frequently indicated for asynchronous motors, and they need not, therefore, be alluded to here. For $K$ and $K_{t}, \mathrm{I}$ have employed for several years the most direct method, which consists in determining for the same machine curves of distribution of magnetic potential, and of the flux in the air-gap, assuming that the armature is traversed by a known current either active or reactive. By taking into account the position of the pole pieces in these two cases, and their form, as well as that of the slots, the reactions may be determined with sufficient precision.

Let us consider, for example, the case of three-phase currents: the three phases occupy in a double field six slots, or groups of slots, and at the passage of each slot, the magnetic potential along the entrefer undergoes a sudden positive or negative increase equal to $0.4 \pi$ multiplied by the number of ampere-turns $\frac{N_{i}}{6}$ contained in the slot. It suffices to mark off on a straight line, representing the development of the circumference of the armature, lengths equal to the distance between the axes of the slots, and on successive ordinates, the variations of the magnetic potential thus calculated. The horizontal 
mean line of the curve so obtained is then traced, which indicates the zero of magnetic potential. The fluxes will be at every point proportional to the ordinate of the curve from the zero point, and inversely proportional to the reluctance per unit of surface corresponding to the abscissa considered. For simplicity, the reluctance may first be assumed constant, and account is only taken of its variations in order to arrive at a definite correction for a second approximation. To simplify the calculation, there is attributed to the maximum amplitude of the polyphase currents an arbitrary value $I_{0}$, and it is supposed that the currents follow a sinsuoidal law. Since the form of the curves is reproduced for every one-sixth of a period in the three-phase currents (or for one-fourth of a period in two-phase currents), it suffices to study them during such an interval, and then to outline the extreme forms.

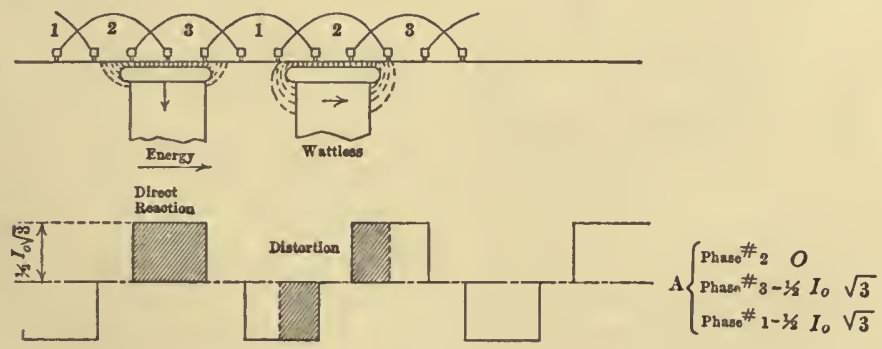

FIG. 8.

Let us take, for example, a three-phase machine with six slots in the field, each containing $N / 6$ wires, calling $N$ the total number of peripicral wires per double field (Fig. 8). The potential produced by each is $0.2 \pi(N / 6) i$, calling $i$ the current which traverses the winding, and it suffices to construct the curve of $i$, to which that of the potentials should be proportional. We take two positions; one for which the current is nil in the slots I and 4 and equals $\pm \frac{\sqrt{3}}{2} I_{0}$ in the others; the other position for which the current is equal to $I_{0}$ in the slots $\mathrm{I}$ and 4 and equal to $(\mathrm{r} / 2) I_{0}$ in the others. The curves proportional to the potential thus obtained are respectively represented in Figs. 8 and 9. On these figures are added in position and in magnitude the outlines of the field-magnet poles in the two positions; in full lines the position corresponding to the watt current, that is to say, a pole axis coinciding with the middle of the curve of potential; 
and in dotted lines the position corresponding to the reactive current, that is to say, the axis of the pole facing the zero of the curve.

The reaction is then deduced from the figure by determining the mean useful ordinate of the curve. Theoretically this ordinate would be obtained by evaluating the area of the shaded curve situated in front of the pole, and dividing this by the breadth of the pole; but the result so obtained is not practically useful, because it takes no account of the expansion of the lines of force, which greatly broadens the flux, particularly as the air-gap is made larger and the angles of the pole pieces are more rounded. To determine the direct reaction, one must take instead of the breadth of the pole, the breadth of the field-magnet flux which issues from it; and to determine the mean ordinate in this breadth. A similar determination is made for the transyerse reaction. It must be observed that the flux which forms

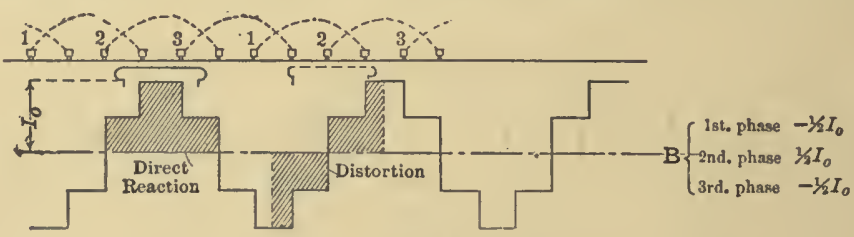

FIG. 9.

it is established not only under the poles, but also around them, although with a lesser density. Consequently, this flux occupies a greater breadth in which the mean reaction should be determined. There is, therefore, a large individual liability to error in the appreciation of these reactions, and this should give preference to the complete method of operation here indicated for the employment of theoretical coefficients, which do not take account of the special conditions in each machine. If the breadth of the flux is equal, for example, to the pitch, Figs. 8 and 9 show the mean ordinates $\frac{2}{3}\left(I_{0} \frac{\sqrt{3}}{3}\right)$ and $\frac{2}{3} I_{0}$ for the direct reaction, and similarly for the transverse reaction. The values give those of the coefficients $K$ and $K_{t}$ themselves, if the ampere-turns obtained are compared with the ampere-turns which would be obtained with the three bobbins united in a single pair of slots and traversed by a current $I_{0}$. The curve of potential gives $\frac{2 N}{6}\left(\cdot \frac{2}{3} I_{0} \frac{\sqrt{3}}{2}\right)$ instead of $\frac{N}{2} I_{0} \times 3$. The ratio gives the coefficient $K=\frac{2}{3}\left(\frac{2}{3} \frac{\sqrt{3}}{2}\right)=0.384$. 
Thus the coefficients $K$ and $K_{t}$ are obtained simply by taking $\frac{2}{3}$ of the mean ordinates. For two-phase currents, one would similarly take $\frac{2}{2}$, that is to say, unity.

If instead of one slot per phase, there were several, $n$ for example, the mean ordinate would be first divided by $n$.

In this manner, the following figures would be obtained:

\section{TABLE IV}

\section{EMPIRICAL COEFFICIENTS}

Three-phase winding, with three separate coils per pair of poles.

\begin{tabular}{|c|c|c|c|c|c|c|}
\hline \multirow{2}{*}{$\begin{array}{l}\text { Ratio } \frac{\delta}{d} \text { of the breadth } \\
\text { of flux to that of polar } \\
\text { pitch. }\end{array}$} & \multicolumn{3}{|c|}{ Coefficient $K$ (Direct). } & \multicolumn{3}{|c|}{$\underset{\text { verse). }}{\underset{\text { Coefricient }}{K_{t}} \text { (TRans- }}$} \\
\hline & Pos. I. & Pos. 2. & Mean. & Pos. I. & Pos. $x$. & Mean. \\
\hline I. & $0.3^{8} 5$ & 0.444 & 0.419 & $\circ .3^{8} 5$ & 0.444 & 0.419 \\
\hline$\frac{2}{3}$. & 0.577 & 0.500 & $0.53^{8}$ & 0.288 & 0.333 & 0.310 \\
\hline$\frac{1}{2}$. & 0.577 & 0.555 & $0.5^{66}$ & o. I92 & 0.333 & 0.2625 \\
\hline
\end{tabular}

In practice an alternator is rarely found where the flux occupies less than two-thirds of the pitch, and besides in this case a winding of twelve bobbins with six short slots should be taken, in my opinion, instead of the ordinary winding, as will be mentioned further on.

The coefficients of self-induction $l$ and $l^{\prime}$, corresponding to the two reactions, are deduced from the values of $K$ and $K_{t}$ by evaluating the corresponding fluxes and the E.M.F.'s which they induce in the windings themselves by means of the ordinary formulas. From this, calling $k$ the winding factor, the mean value of $l$ which takes account of the reduction, by distribution of the wires, in the E.M.F. produced in the winding by a sinusoidal flux, ${ }^{1}$

$$
l=\frac{4 \pi K_{\ell} k\left(\frac{N}{2}\right)^{2} \mathrm{10}^{-9}}{q R_{t}} ; \quad l^{\prime}=\frac{4 \pi K k\left(\frac{N}{2}\right)^{2} \mathrm{IO}^{-9}}{q R_{d}}
$$

${ }^{1}$ See in particular the coefficients in my above-mentioned analysis of the rotating magnetic fields, Éclairage Électrique, 1895. 
$q$ being the number of phases (here 3 ), $R_{t}$ and $R_{d}$ the reluctances of the transverse and direct circuits respectively. ${ }^{1}$

These reluctances are determined from the drawing of the machine, taking into account the real path of the lines of force and the saturation of the parts through which they pass, particularly the teeth of the armature, the polar horns, the cores, the yokes of the field-magnets, etc.

If, instead of alternate poles, the machine carries poles of the same name (homopolar inductor), $K$ and $K_{t}$ may again be determined by the preceding methods, drawing only one inductor-pole for two poles of the armature. It results from this that theoretically the reactions would give rise to coefficients 50 per cent less than in the ordinary case. In practice, however, this is far from being the case, because of the very considerable expansion of the flux of the armature in the large spaces existing between the field-magnet poles. The direct flux reaction and particularly the transverse reaction is, therefore, much larger than if they were produced only by the action of the poles; so that finally the reactions are scarcely reduced more than 25 per cent. The stray fields are, moreover, very large in this type of machine, and every expansion of the field-magnet flux beyond the breadth of the pitch produces a hurtful inverse E.M.F. The induction-density in the air-gap should finally be doubled at least, to produce the same useful flux on open circuit. From all the above it follows that homopolar machines are of little advantage and are almost abandoned.

To completely take into account the practical values of the coefficients, we shall consider again the case of three-phase machines with six coils per field, first concentrated into one pair of slots per coil, and then spread uriformly (or to a large number of slots each) in order to occupy the entire circumference of the armature.

Figs. II and I2 represent the curves of magnetic potential obtained in the two hypothetical cases with long bobbins disposed as shown diagrammatically in the figure. The two curves correspond to the same hypothesis as above for currents, and Table II represents the separate mean values obtained for $K$ and $K_{\imath}$ from these curves.

${ }^{1}$ In unsaturated alternators, if one calls $e$ the single air-gap, $s$ the polar surface, and $d$ the coefficient of enlargement of the flux, the equation is approximately obtained $\frac{\mathrm{I}}{R_{d}}=\frac{s(\mathrm{I}+d)}{2 e}$. 

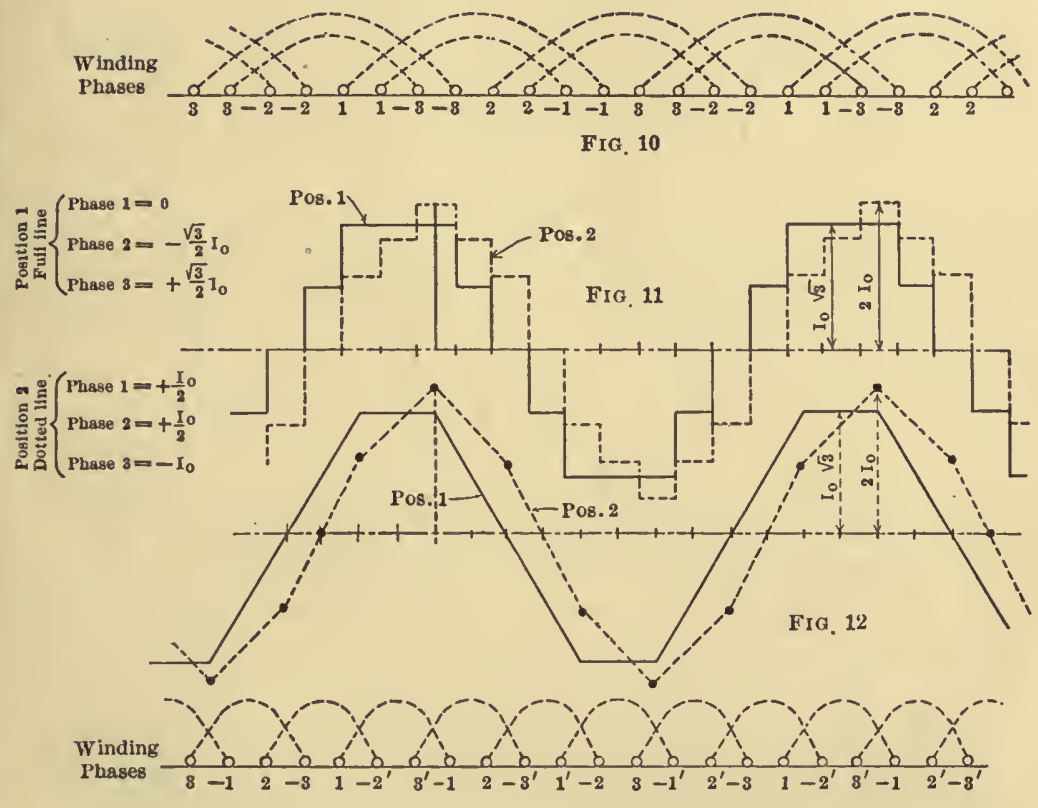

FrG. 13
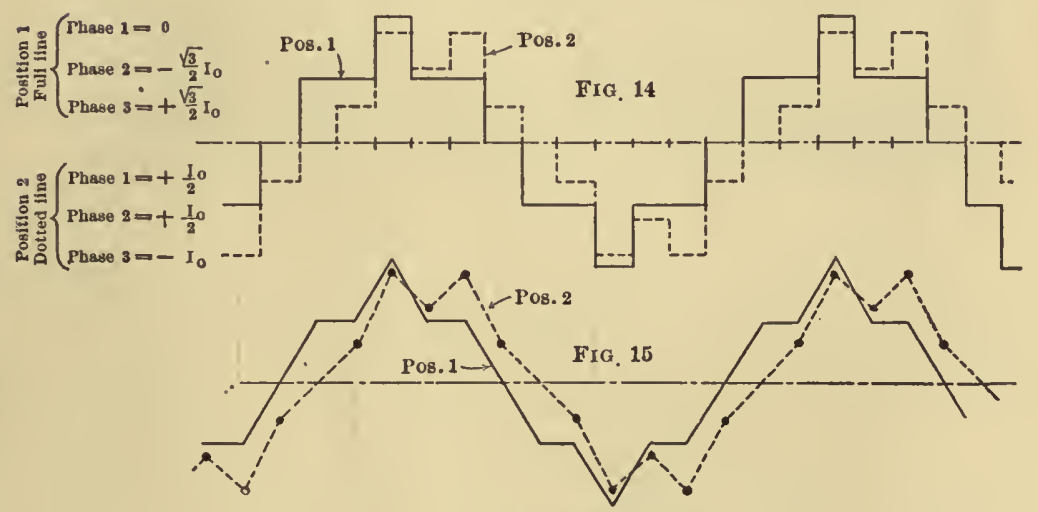

Frgures, 10 To 15 
But a winding with six coils may also be realized symmetrically following the plan of Fig. 16, from which two new curves $16 a$ and $16 b$ are obtained. The table also indicates the value of the coefficients thereby deduced.

\section{TABLE V}

\section{EMPIRICAL COEFFICIENTS}

Three-phase winding with three separate coils per pair of poles.

\begin{tabular}{|c|c|c|c|c|c|c|c|}
\hline \multirow{2}{*}{\multicolumn{2}{|c|}{ Ratio $\frac{\delta}{A}$}} & \multicolumn{3}{|c|}{$K}$. & \multicolumn{3}{|c|}{$K_{t}}$. \\
\hline & & Pos. I. & Pos. 2. & Mean. & Pos. 1. & Pos. 2. & Mean. \\
\hline \multirow{3}{*}{ Long coils } & 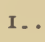 & $0.3^{8} 5$ & $0.3^{89}$ & $0.3^{87}$ & $0.3^{85}$ & 0.389 & $0.3^{87}$ \\
\hline & $\frac{2}{3}$. & $0.5 \circ 5$ & 0.500 & 0.5025 & 0.288 & $0.29 \mathrm{I}$ & 0.2915 \\
\hline & $\frac{1}{2}$. & 0.577 & 0.555 & 0.566 & o. $19^{2}$ & 0.222 & 0.207 \\
\hline \multirow{3}{*}{ Short coils } & I. . & 0.2886 & 0.2777 & 0.283 & 0.2886 & 0.2777 & 0.283 \\
\hline & $\frac{2}{3}$. & 0.360 & 0.375 & 0.3675 & 0.216 & 0.208 & 0.212 \\
\hline & & $0.3^{8} 5$ & 0.444 & 0.415 & 0.192 & O. II I I & $0.15^{2}$ \\
\hline
\end{tabular}

\section{TABLE VI}

\section{EMPIRICAL COEFFICIENTS}

Three-phase distributed winding, 3 or 6 long coils per pair of poles.

\begin{tabular}{|c|c|c|c|c|c|c|}
\hline \multirow{2}{*}{$\begin{array}{l}\text { Ratio } \frac{\partial}{\Delta} \text { breadth of } \\
\text { flux to polar pitch. }\end{array}$} & \multicolumn{3}{|c|}{$K}$. & \multicolumn{3}{|c|}{$K_{\ell}}$. \\
\hline & Pos. $t$. & Pos. 2. & Mean. & Pos. 1 . & Pos. 2. & Mean \\
\hline$\frac{1}{1}$. & $0.3^{8} 5$ & $0.3^{89}$ & $0.3^{87}$ & $0.3^{85}$ & $0.3^{89}$ & $0.3^{87}$ \\
\hline$\frac{2}{3}$. & 0.505 & 0.500 & 0.5025 & 0.2886 & 0.2916 & 0.200 \\
\hline$\frac{1}{2}$. & 0.553 & $0.54 \mathrm{I}$ & 0.547 & 0.216 & 0.236 & 0.236 \\
\hline
\end{tabular}

Here again it is seen that the direct reactions increase while the transverse reactions diminish when the breadth of the flux (larger than the pole) diminishes. It is moreover determined that the reac- 
TABLE VII

\section{EMPIRICAL COEFFICIENTS}

Three-phase winding with 6 short distributed coils per double field.

\begin{tabular}{|c|c|c|c|c|c|c|}
\hline \multirow{2}{*}{ Ratio $\frac{8}{4}$} & \multicolumn{3}{|c|}{$K$. } & \multicolumn{3}{|c|}{$K_{\ell}$} \\
\hline & Pos. I. & Pos. 2. & Mean. & Pos. 1. & Pos. 2. & Mean. \\
\hline$\frac{1}{1} \ldots$ & 0.289 & 0.277 & 0.283 & 0.289 & 0.277 & 0.283 \\
\hline$\frac{2}{3}$. & 0.360 & 0.375 & 0.3675 & $0.2 \mathrm{I} 6$ & 0.208 & $0.2 \mathrm{I} 2$ \\
\hline$\frac{1}{2}$. & $0.3^{8} 5$ & 0.444 & $0.4 \mathrm{I} 45$ & ०. $19^{2}$ & 0.137 & ०. 1645 \\
\hline
\end{tabular}

tions are markedly reduced by the employment of short coils. But it may be readily shown that with a sinsuoidal field-magnet flux of a breadth equal to the pitch, the E.M.F. induced in the winding is reduced approximately in the same ratio. In fact, the mean breadth of the short bobbins, Fig. r6, is $\frac{1}{4}$ of the pitch, while the mean breadth

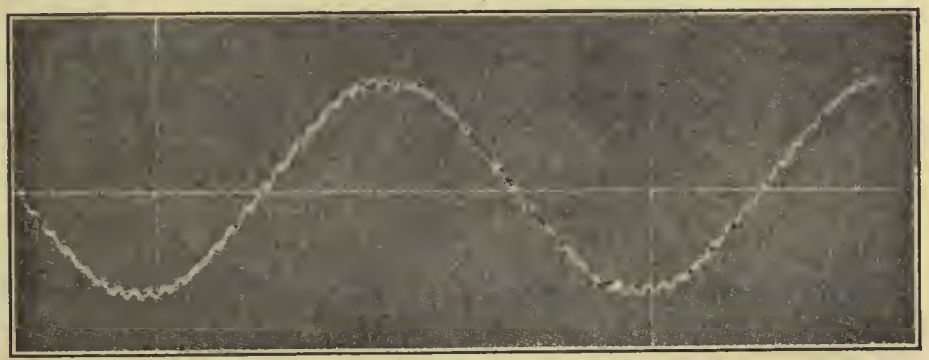

FIG. I6.

of the long bobbins, Fig. Io, is $\frac{1}{2}$ the pitch. The straddling arrangement of coils in Fig. ro involves only the coefficient of reduction $2 \cos \left(\frac{\pi}{12}\right)$ (

E.M.F. in the same ratio as the flux linkage, that is to say, by the coefficient $k=\sin \frac{\pi}{4}=\frac{\sqrt{2}}{2}$. The two E.M.F.'s are then in the ratio $\frac{E \text { short bobbins }}{E \text { long bobbins }}=\frac{0.707}{0.966}=0.73$. 
But the ratio of the coefficients $K$ and $K_{t}$ of the two windings gives approximately the same figure. It is to be observed in this connection

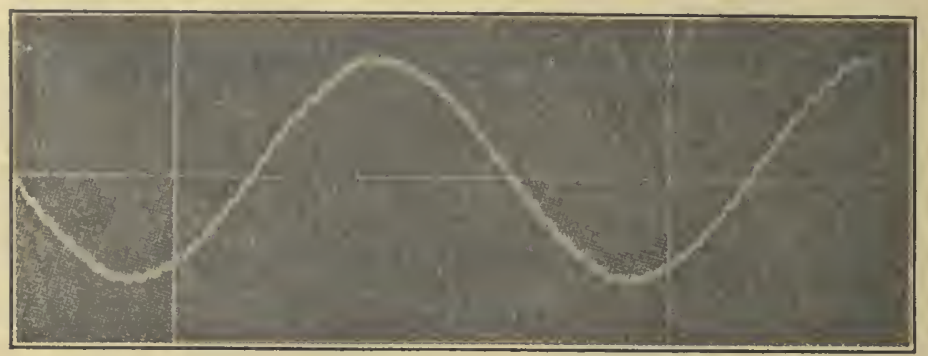

Fig. $16 a$.

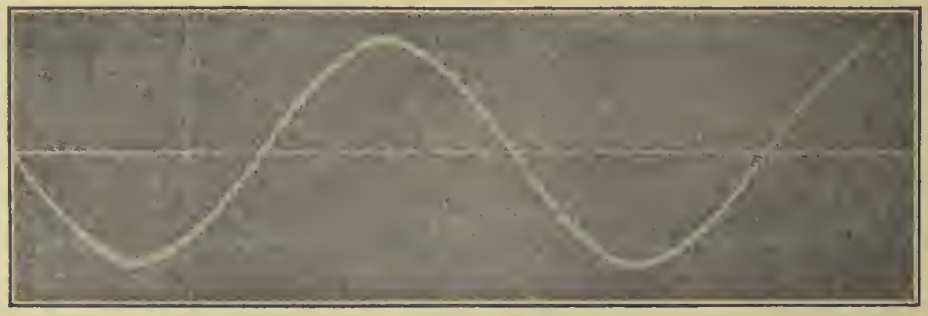

Fig. $16 b$.

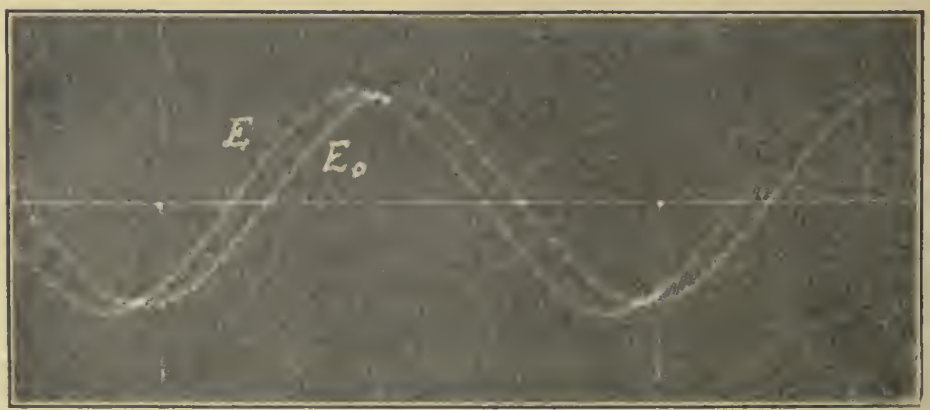

Fig. $16 c$.

that for the case of a flux having a breadth equal to the polar pitch, the coefficient $K$ is substantially equal for these two windings, that is, to $k$ of each winding multiplied by $\left(\frac{2}{\pi}\right)^{2}$, which verifies the general 
law formerly announced and which is alluded to above. ${ }^{1}$ Hence it follows that the fluxes produced by multiple-coil windings differ but little from the mean value of the theoretical fiuxes, and approach the more nearly as the sections are the more numerous, and also as the local variations or fluctuations of the curves between the extreme forms I and 2 of the appended figures are damped out by the Foucault currents of the neighboring pole pieces. The energy expended in these Foucault currents, being supplied by the armature, is represented by an augmentation of its apparent resistance $r^{\prime}$.

The reactions of two-phase armatures would be found in a similar manner, and it will not be necessary to reproduce them more in detail. Moreover, two-phase machines are more and more becoming supplanted by three-phase, and the latter present reactions of much smaller fluctuations and a better utilization of materials, just as threephase motors are superior, from this standpoint, to two-phase motors.

Comparison with Theoretical Coefficients. The theoretical coefficients are easy to establish in the case where a sinusoidal flux is assumed and the harmonics are suppressed. ${ }^{2}$ It is then demonstrated that the magnetic potential produced by a polyphase winding of $q$ phases is independent of the number of phases and depends only upon the total number of wires $N$ per double field, and that it is represented by a sinusoid whose amplitude is $2 N I_{0}$. The mean potential in the air-gap is therefore $\frac{2}{\pi} 2 N I_{0}$ and the equivalent mean magnetomotive force producing the reactive flux on closed circuit

$$
=\frac{0.4}{\pi} 2 N I_{0}=\left(\frac{4}{\pi}\right)^{2} \times 0.4 \pi \frac{N}{2} I_{0}
$$

that is to say, $(4 / \pi)^{2}$ of the magnetomotive force which will give the same turns if they coincide in position and phase. In this case the sinusoid of potential (Fig. I7) is entirely used and the direct reaction is proportional to the mean ordinate of the area $A_{3} B$, and the transverse reaction to that of the area $3 H B H^{\prime}$ io. If, on the contrary,

${ }^{1}$ In alternators with distributed windings (Figs. I2 and I5), the diagrams of windings ro and $\mathrm{I}_{3}$ respectively may be employed by assuming that each coil is replaced by a zone of wires occupying along the air-gap a breadth of $\frac{1}{12}$ of the field, of $\frac{1}{8}$ of the polar pitch, and having as median line the old outline of the single bobbin which it replaces.

2 See my above-mentioned memoir of 1899 upon "Rotating Magnetic Fields," see also Arnold and la Cour's "Vorausberechnung der Ein und Mehrphasenstromgeneratoren." Stuttgart, Igor. 
the reactive flux only occupies a part $\delta$ of the pitch instead of $J$, as indicated by the dotted intersecting lines in the figure, the reactions will be proportional to the mean ordinates of the areas 12345 and $67 B 89$ respectively, limited to the breadth of the flux (which may be different moreover for the transverse flux from what it is for the direct flux. By integrating the area of the sinusoid from $\mathrm{H}_{3}$ on to 54 one finds

$$
\begin{aligned}
& \text { Ordinate of area } 12345=\frac{\Delta}{\delta} \sin \left(\frac{\pi}{2} \frac{\delta}{\Delta}\right) \times \frac{2}{\pi} I_{0} . \\
& \text { Ordinate of area } 67 B 89=\frac{\Delta}{\delta}\left[\mathrm{I}-\cos \left(\frac{\pi}{2} \frac{\delta}{\Delta}\right)\right] \times \frac{2}{\pi} I_{0} .
\end{aligned}
$$

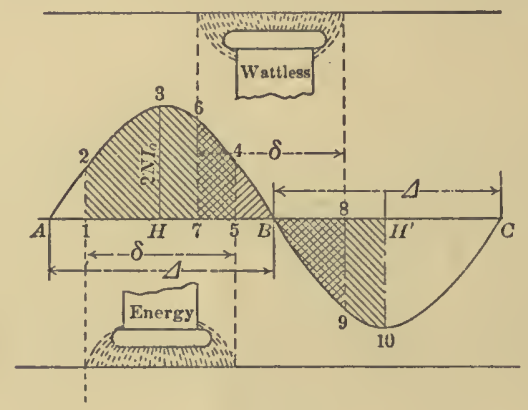

Fig. I7.

The coefficients which apply to the ampere-turns, resulting from a restriction of the flux, are then respectively for $K$ and $K_{t}$

$$
c=\frac{\Delta}{\delta} \sin \left(\frac{\pi}{2} \frac{\partial}{\Delta}\right) \text { and } c_{t}=\frac{\Delta}{\delta}\left[\mathrm{I}-\cos \left(\frac{\pi}{2} \frac{\delta}{\Delta}\right)\right],
$$

and have the following values for example (not including $k$ )

$$
\begin{aligned}
& \text { for } \frac{\delta}{\Delta}=\frac{3}{4} ; \quad c=\mathrm{I} .232 ; \quad c_{t}=0.8 \mathrm{I} 7 \text { from which } K=\mathrm{I} .232\left(\frac{2}{\pi}\right)^{2} k \\
& K_{t}=0.8 \mathrm{I} 7\left(\frac{2}{\pi}\right)^{2} k \\
& \text { for } \frac{\delta}{4}=\frac{2}{3} ; \quad c=\mathrm{I} .299 ; \quad c_{t}=0.75 \\
& K=\mathrm{I} .299\left(\frac{2}{\pi}\right)^{2} k \\
& K_{t}=0.75\left(\frac{2}{\pi}\right)^{2} \\
& \text { for } \frac{\delta}{\Delta}=\frac{1}{2} ; \quad c=\mathrm{I} .4 \mathrm{I} 4 ; \quad c_{t}=0.586 \\
& K=\mathrm{I} .4 \mathrm{I} 4\left(\frac{2}{\pi}\right)^{2} k \\
& K_{t}=0.586\left(\frac{2}{\pi}\right)^{2} k
\end{aligned}
$$




\section{TABLE VIII}

\section{THEORETICAL COEFFICIENTS}

Winding with three coils per pair of poles.

\begin{tabular}{|c|c|c|c|c|}
\hline \multirow{2}{*}{ Ratio $\frac{\partial}{d}$} & \multicolumn{2}{|c|}{ Separate Colls. } & \multicolumn{2}{|c|}{ Distributed Colls. } \\
\hline & $K$. & $K_{t}$. & $K$. & $K_{t}$ \\
\hline$\frac{1}{2} \ldots$ & 0.405 & 0.405 & $0.3^{8} 59$ & $0.3^{8} 59$ \\
\hline$\frac{2}{3}$. & $0.5^{26}$ & 0.304 & 0.501 & 0.2897 \\
\hline$\frac{1}{2} .$. & $0.57^{2}$ & 0.237 & 0.544 & 0.2257 \\
\hline
\end{tabular}

TABLE IX

THEORETICAL COEFFICIENTS

Winding with 6 coils.

\begin{tabular}{|c|c|c|c|c|c|}
\hline \multirow{2}{*}{ i } & \multirow{2}{*}{ Ratio $\frac{\delta}{d}$} & \multicolumn{2}{|c|}{ Separate Colls. } & \multicolumn{2}{|c|}{ Distributed Colls. } \\
\hline & & $K$. & $K_{t}$. & $K$. & $K_{t}$. \\
\hline \multirow{3}{*}{ Long coils... } & $\int \frac{1}{1}$. & $0.39 \mathrm{I}$ & $0.39^{1}$ & 0.3859 & 0.3859 \\
\hline & $\frac{2}{3}$. & 0.508 & 0.293 & 0.501 & 0.2897 \\
\hline & 17 & $0.55^{2}$ & 0.228 & 0.544 & 0.2257 \\
\hline \multirow{3}{*}{ Short coils... } & $\frac{1}{1}$. & 0.286 & 0.286 & 0.2825 & 0.2825 \\
\hline & $\frac{2}{3}$ & 0.371 & 0.215 & 0.3665 & 0.212 \\
\hline & $\frac{1}{2}$. & 0.404 & 0.167 & 0.399. & 0.164 \\
\hline
\end{tabular}

Since these figures must also be modified in every case by the coefficient $k$ corresponding to the E.M.F., as has been explained above, it is evident that they will differ but little from those of Table II. But it is not the less necessary to determine, from a drawing of the machine, the breadth of the flux before applying it in these formulæ, and therefore to correct the reactions in order to take account of the saturation of the different parts of the armature and of the pole pieces.

This correction is made by assuming the magnetic conditions which are approximately attained in the machine at full load. The 
mean flux-density is then known which must be developed in the air-gap, the teeth, and the pole pieces, and, moreover, one has from the curves of potential the value of the magnetomotive forces acting at all points of the air-gap. From this may be deduced the real flux-density at every point, and consequently the true variation of the total flux produced by the reaction. These expressions of selfinductions given above should be consequently replaced by the integrals of the form

$$
l=\frac{N}{q_{2} \sqrt{2} I_{0}} \int\left(0.4 \pi \frac{N}{6} \frac{y b}{R_{x}}\right) d x
$$

indicating by $x$ the abscissa, and by $y$ the ordinate of the curve, and $R$ the reluctance per unit of surface at this point. $b$ represents the length of the armature, $q$ the number of phases, $N$ the number of peripheral wires per field.

Case of Single-phase Alternators. The problem of the reactions of single-phase alternators is more complex than that of polyphase alternators, as will be seen. It does not appear to have been fully understood by the authors who have previously treated it. It may be analyzed by the same method as that which I have formerly developed for asynchronous motors, ${ }^{1}$ but taking into account this important difference, that, in general, the rotating reactions (rotating magnetic field with respect to the armature, which we suppose fixed), are suppressed in motors by the short-circuited windings on the rotor, but not, in alternators, except in the case where they are furnished with massive poles, or especially with the damping wirdings of Leblanc (which, however, only give a partial suppression).

I have shown that each coil of a single-phase armature produces a reactive flux capable of being decomposed in space into sinusoidal harmonics, of which the first, the only one which need be considered in practice, has for amplitude $\frac{0.4 \pi n I}{R}$, denoting by $n I$, the ampereturns of the coil, and by $R$, the reluctance of the magnetic circuit traversed by the flux which it produces. In accordance with the theorem of Leblanc, I decompose this pulsating sinusoid into two rotating sinusoids of one-half amplitude: one turning synchronously with the rotor, and not displaced therefore. with respect to the field-poles; it provides only a fixed reaction; the other rotate

\footnotetext{
"Blondel, "Properties of Rotating Magnetic Fields," Éclairage Électrique, May, 1908 .
} 
in the opposite direction with the same speed and is therefore displaced with respect to the field magnets with a speed double that of synchronism; this gives rise to a pulsating flux in the field magnets of frequency double that of the e.m.f. induced in the armature, as I have also shown experimentally. ${ }^{1}$ This reaction is somewhat weakened by the currents which it produces in the closed circuit of excitation, but it is not completely extinguished and produces therefore an e.m.f. of normal frequency in the armature which should be taken into consideration. But, while the fixed reaction may be analyzed, exactly as in polyphase alternators, into direct and transverse reactions, the parasitical rotating reaction is effected directly through the field magnets, as well as transversely through the pole pieces, and may be represented, consequently, by a mean coefficient of self-induction similar to the self-induction of the stray field $\omega s$, to which it is added.

It may be directly demonstrated by calculations that this analysis readily lends itself to the interpretation of the facts, ${ }^{2}$ and that a single-phase alternator whose armature at rest presents a self-induction

'Blondel, "Photographic Record of Periodic Curves," Lumière Électrique, August, I89x.

${ }^{2}$ In fact, the self-induction of the armature $L$, varying between the two values $\lambda$ and $\lambda^{\prime}$ according to position, may be represented by an expression

$$
L=A+B \cos 2 \omega t+\omega s
$$

and abbreviating,

$$
A+B=\lambda^{\prime} ; A-B=\lambda,
$$

indicating always by $s$ the inductance of the stray fields in the slots. Let $e=E_{0} \sin \omega t$ be the internal E.M.F., and $i=I_{0} \sin (\omega t-\psi)$ the strength of the current, and there is immediately obtained as the difference of potential at the terminals of the machine the following expression:

$$
u=e-r i-L \frac{d i}{d t}-i \frac{d \lambda}{d t}-\omega s \frac{d i}{d t} .
$$

Replacing $e, L, i$ by their values, and neglecting a term $\frac{B \omega}{2} \cos (3 \omega t-\psi)$ which produces an upper harmonic, there remains $u=E_{0} \sin \omega t-r I_{0} \sin (\omega t-\Psi)-\omega\left(A-\frac{B}{2}\right) I_{0} \cos \Psi \cos \omega t$

$$
-\omega\left(A+\frac{B}{2}\right) I_{0} \sin \Psi \sin \omega t-\omega s I_{0} \cos (\omega t-\Psi)
$$

$=E_{0} \sin \omega t-r I_{0} \sin (\omega t-\Psi)-\frac{\left(\omega \lambda+\lambda^{\prime}\right)}{4} I_{0} \cos (\omega t-\Psi)-\frac{\omega \lambda}{2} I_{0} \cos \Psi \cos \omega t$

$$
-\frac{\omega \lambda^{\prime}}{2} I_{0} \sin \Psi \sin \omega t-\omega s I_{0} \cos (\omega-\Psi) \text {. }
$$

The values of $\frac{\lambda}{2}$ and $\frac{\lambda^{\prime}}{2}$ representing that which we have uniformly denoted by $l$ and $l^{\prime}$ for all the machines in the construction of the diagrams. 
$\lambda$ when the poles are crossed, and an inductance of $\lambda^{\prime}$ when they are coincident, behaves under load, when the real dephasing is $\psi$ for example, as though the active current $I \cos \psi$ traversed an inductance $\frac{\lambda}{2}$, the reactive current $I \sin \psi$ an inductance $\frac{\lambda}{2}$, and the total current $I$ a parasitic inductance $\frac{\lambda+\lambda^{\prime}}{4}$ equal to the mean of the two preceding.

In order to take into account the Foucault currents of the armature produced by the rotating reaction, it is sufficient to apply a reducing coefficient $m$ to its inductance, which is less than unity, evaluated according to the conditions of 'construction, and at the same time to increase the apparent resistance of the armature in accordance with the energy lost in these Foucault currents, since it is furnished by the armature. It is for this reason that we attribute to this resistance a value $r^{\prime}>r$ in all of our diagrams.

If the field magnets or the armature of the alternator are saturated, the direct and transverse self-inductances will be replaced by equivalent back ampere-turns, again calculated as in my theory of rotating field: the sinusoid of the amplitude $0.2 \pi n I$ presents the mean ordinate $\frac{2}{\pi}$ times smaller and is consequently equivalent to $\frac{n I}{10}\left(\frac{2}{\pi}\right)^{2}$ ampereturns. The value of $K$ and $K_{t}$ will therefore be $\left(\frac{2}{\pi}\right)^{2}$ for a single bobbin if it has the same breadth as the flux. If the winding comprises several straddling coils, the coefficients should be multiplied by the straddling factor of the winding $k$; finally if the flux of the poles is narrower than the pitch, it should be multiplied by the factors $\frac{\Delta}{\delta} \sin \left(\frac{\pi}{2} \frac{\hat{\Delta}}{\Delta}\right)$ and $\frac{\Delta}{\delta}\left[\mathrm{I}-\cos \left(\frac{\pi}{2} \frac{\delta}{\Delta}\right)\right]$, respectively the coefficient of direct reaction and the coefficient of distortion for the conditions analyzed above. ${ }^{1}$

${ }^{1}$ It is of course easy to pass from a single-phase machine to a polyphase machine of two phases, observing that each phase gives a fixed reaction and a rotating reaction of the same amplitude. I have shown in my theory of rotating fields, already alluded to, that the fixed reactions unite in space and are added algebraically while the $q$ rotating, parasitic reactions give rise to a resultant zero. The coefficients $K$ and $K_{\ell}$ are then themselves theoretically expressions in all the machines independent of the number of phases $(N$ designating always the total number of peripheral wires); but the rotating, parasitic self-inductance disappears in polyphase machines. In this manner the return is made to the theoretical coefficients of the polyphase machines. 
To sum up, the case of the single-phase alternator should then be treated exactly by the same general formulæ, the same constructions, and the same diagrams as in the case of a polyphase alternator, but under the condition of considerably increasing the stray flux $\omega s I$, adding thereto a term representing the parasitic rotating inductance. The coefficient $\omega s$ is thus replaced by $\omega\left[s+m\left(\frac{l+l^{\prime}}{2}\right)\right]$ values $l$ and $l^{\prime}$ are calculated by the theoretical coefficient of polyphase machines (see p. 263) (taking into account the saturation of the circuits by the values given to $R$ and $R^{\prime}$, as has already been seen above). In this manner a coefficient $m$ of reduction will be determined less than unity, the more or less marked suppression of the parasitic rotating inductance by the Foucault currents induced in the surrounding non-laminated metallic masses, and in some cases in special damping circuits.

Consequences from the Point of View of the Construction of Alternators for Good Regulation. The theory and the calculation for the reactances just as they have been above analyzed, lend themselves to the discussion of the construction of alternators much better than the old methods. We will proceed to give a few examples of such applications.

(A) In so far as concerns the employment of short bobbins, (generally abandoned by reason of the disfavor thrown upon them by windings of only three coils per field, which give terrible pulsations), the winding of six short bobbins which I. have indicated (Fig. I3) may be compared with the ordinary winding (Fig. Io) by means of the coefficients calculated above. The relations between the $K$ of the two being the same as between the respective coefficients $k$ of the E.M.F., it is evident that the advantage of the short bobbins from the point of view of reactions, involves a loss of E.M.F. in exactly the same proportion. To re-establish the desired value of the latter, the number of turns of the armature must either be increased, consequently re-establishing the same reaction, or the flux density in the air-gap must be increased, and consequently the ampere-turns of the fieldmagnets as well as the losses by Foucault currents. The two windings are therefore equivalent from the constructive standpoint when the field-magnet flux occupies the entire polar pitch. However, short bobbins may be treated more rationally by reducing the breadth of the field-magnet flux to two-thirds of the field-magnet pitch in such a manner that the flux shall be entirely utilized in the coil. Further 
increasing the flux-density, an e.m.f. is obtained equal to that in long bobbins, and the direct reaction remains in the same ratio with respect to the e.m.f. while the transverse reaction is reduced. The winding of Fig. 13, with a flux having the value of two-thirds of the pitch, is then that which for a given ratio of counter-ampere-turns of the armature to the ampere-turns of excitation, produces the smallest transverse reaction. This reduction is of considerable importance.

(B) As to the methods of reducing reactions, it results from the preceding that there is no means of reducing the ratio $\frac{K}{k}$. The only means of improving the regulation of alternators are therefore, first, to saturate the field-magnet circuit (which augments the m.m.f. and reduces the variations of e.m.f. at the armature terminals as a function of the reactive current); second, to increase the air-gap, which is less effective; and third, to reduce the transverse reaction, which has the effect of diminishing the dephasing of the diagrams, and consequently the direct reaction, which is proportional to the reactive current. An alternator which would not have transverse reaction, would have nothing to fear from direct reaction, even if it were enormous.

The methods of reducing the transverse reactions alone are: First, the reduction of the breadth of the flux accompanied by an augmentation of the flux-density in the air-gap, re-establishing the same field-magnet flux at the expense of the increase of the flux-density; but in that case the same result would be obtained by a simple increase of the air-gap, without an increase in the loss of energy in the teeth; second, the saturation of the polar horns when the pole pieces possess them; third, the addition of longitudinal slots in the field-magnets, as in direct-current dynamos. This last method is very effective when the field magnets are saturated and the slots occupy their entire length and are continued partially into the yoke; the reduction of distortion thus obtained involves generally a reduction of the total flux, because the mean permeability of the field magnet is reduced by the inequality of the m.m.f.'s established between its two halves; but the augmentation of excitation which results is negligible in comparison with the diminution obtained in the reactive current by the reduction of dephasing.

(C) As to the comparison between single-phase and polyphase alternators, it is seen that not only do single-phase alternators utilize less effectively their materials for the production of energy, because 
their armature surface is less utilized for e.m.f., but also their armature reaction gives rise to a hurtful, parasitic self-induction which does not exist in polyphase alternators and which reduces their good regulation. This parasitic inductance can only be partially suppressed at a cost of the expenditure of energy equivalent to a considerable augmentation of the apparent resistance of the armature.

\section{RÉSUMÉ ANd CoNCLUSION}

To sum up, it has been established in this paper that the theory of two reactions of the armature admits of analyzing the phenomena of alternators with greater precision than the old theories, besides having the advantage of referring them to conditions similar to those of direct-current machines. Simple diagrams are given applicable to alternators of saturated field magnets and unsaturated armatures (Fig. 4) and even to saturated armatures (Fig. 7) without involving a complicated correction.

It has been indicated how to calculate the coefficients of reaction, not only theoretical, but also actual values, by means of curves of magnetic potential in the air-gap. Interesting relations have been established between these coefficients and those of the induced e.m.f.

Comparisons have been established between the different types of winding, and the advantages possible for a special winding with short bobbins have been made evident.

It has been shown that single-phase alternators may be treated by the same methods, adding, however, to the inductance of the stray field a parasitic inductance which does not exist in polyphase machines.

Finally the consideration of the transverse reaction has permitted the discussion of a construction in view of good regulation, showing the interest which attaches to reducing the coefficient of distortion in alternators, and indicating the means of such reduction.

The author hopes that, thanks to simplicity of application, much greater than is often believed, and by its relations with the theory of direct-current machines, this method of calculation (in which he has had practical experience for several years) may be of service to designers and satisfy the need of rational precision in this work. 


\title{
CHAPTER II
}

\section{METHODS OF TESTING ALTERNATORS ACCORDING TO THE THEORY OF TWO REACTIONS 1}

\author{
By Prof. ANDré Blondel, École des Ponts et Chaussées.
}

THE author described in the Bulletin de la Sociêtê des Electriciens in 1892 a method of testing alternators similar to that of Hopkinson for direct-current machines, which permits of studying both their efficiency and their armature reactions, under the same conditions of operation and without a large expenditure of power, under the sole condition that the two alternators shall be similar. This method depends upon the rigid coupling of two similar alternators. Later the author published a variation of the method which does not call for the rigid coupling between the alternators, but which consists in operating the alternator on test as a synchronous motor, by the aid of an auxiliary alternator. ${ }^{2}$ The alternator under test revolves on no-load, as a motor, under the normal current. This method has been designed particularly to determine the efficiency with greater precision than by separating the various losses. The object of the present paper is to complete and yet further perfect this method by pointing out how it may be likewise used for measuring armaturereactions (especially by the employment of two reactions, set forth in the preceding chapter).

Method No. I. When the Rigid Coupling of the two Alternators is Possible. When two similar alternators are available, and when they can be placed side by side so as to be connected rigidly by a short coupling, the following tests may be carried out (Fig. I):

First a certain difference of phase $\alpha$ is provided between the alternators (for example, a phase-difference of $30^{\circ}$, that is to say, one-sixth of a pole, or $45^{\circ}$, that is to say, one-fourth of

${ }^{1}$ A paper presented by the author before the International Electrical Congress at St. Louis in 1904. Reprinted from the Transactions, Vol. I, pp. 620-634.

${ }^{i}$ See La Lumière électrique, 1893. 
a pole. The system of two machines is driven by a measuring motor whose duty it is to furnish the power necessary for satisfying the losses. Between the two alternators $A_{1} A_{2}$ (Fig. I8) whose terminals are connected each to each by very

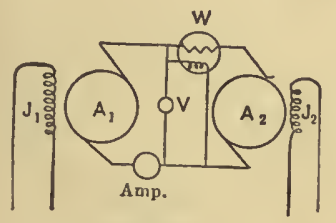

FIG. 18. short couplings of negligible impedance, a volt-meter $V$ is connected across, an ammeter $A$ and a wattmeter $W$ being inserted in series. The figure is drawn upon the supposition of two single-phase machines, but applies equally well to the case of two similar three-phase machines coupled by their three phases, testing upon a single phase, taking care that the phases remain balanced, in spite of the measuring instruments.

Let $U=O B$ (Fig. I9) be the difference of potential observed at the common terminals, $O A_{1}$ and $O A_{2}$ the direction of the generator e.m.f.'s dephused relatively to each other by the angle $\alpha$. By symmetry, the vector $O b$, which represents the current, will also be directed along $O B$, and the line $A_{1} A_{2}$ drawn from $B$ perpendicularly to $U$ and $I$, will represent the double transverse reaction $2 \omega L^{1} I$. There will be a flow of current between the alternators without the production of any external power. The current will be in phase with the E.M.F. at the terminals $U$, as if the alternators supplied a conducting system devoid of inductance; the flux density obtained in the armature will be the same in both alternators, since it gives rise to the same E.M.F. at the terminals $U$. The power furnished by each will be measured by the wattmeter $W$, and the total

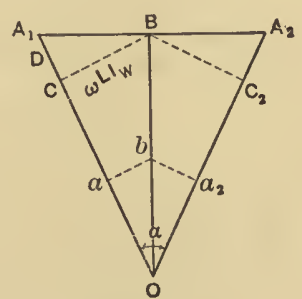

FIG. I9. loss $p$ will be furnished by the method of double-weighing, by means of the measuring motor which drives both alternators. The efficiency will then be the ratio

$$
P_{u}=\frac{U I}{U I+\frac{p}{2}}
$$

To determine the excitations necessary for the two alternators to produce the condition above described, it is sufficient to apply the graphic method of two reactions, as follows (Fig. I9): from the point 
$B$ a perpendicular $B C$ is let fall upon the straight line $O A_{1}$, and the condition is such as if the alternator were delivering power to an inductive system according to the total characteristic of excitation (Fig. 20). The current $I$ is formed of two components: one an active component $O a$, equal to the projection of $I$ upon $O A$, and

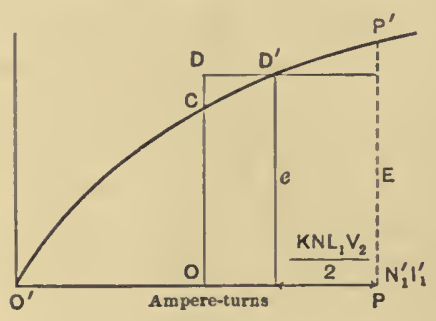

FIG. 20. which gives the transverse reaction $B C$ equal to $\omega L I_{x}$; the other component is a reactive or reactive component $a b$, which gives the fall of potential to be calculated on the characteristic. Let us lay off on this characteristic (Fig. 2C) an ordinate equal to $O C$, and $\mathrm{upon}$ the latter a segment $C D$ equal to the e.m.f. lost by the stray field. From the point $D$ lay off horizontally the counter ampereturns of the armature, and thus will be obtained on the abscissa $O^{\prime} P$ the total ampere-turns necessary for the excitation. The ordinate $P P^{\prime}$ corresponding thereto will represent the e.m.f. $E$ on open circuit necessary for alternator $A_{1}$ and which will be, in general, different from the length of $O A_{1}$ which was represented in Fig. I9.

In the same way the e.m.f. is determined which is necessary for the alternator $O A_{2}$, observing that for the latter the sign of the reactive armature-reaction is changed, as well as the sign of $E=A_{2} \mathrm{O}$, and that, consequently, the armature reaction remains demagnetizing, so that the geometrical construction is identical. . It is, therefore, easy to recognize in advance the equal excitations to be given to the two alternators, in order to satisfy the desired conditions. It should also be determined at the time of the test, by means of the wattmeter, that there is no sensible difference of phase between the current and the e.m.f. Inversely, if this condition were directly realized by adjusting the excitations, it would be possible to deduce from an examination of the diagram the total armature-reactions represented by the abscissa $O P$, indicating the total fall of excitation between the open circuit e.m.f. and the e.m.f. under load. In the latter case, the fall due to the stray field is not separated from that due to armature reaction.

The same diagram gives immediately the value of the transverse 
reaction, since the angle $\alpha$ is known experimentally, and the values of $U$ and $I$ can be consequently measured. This gives

$$
A_{1} B=\omega L^{1} I=U \tan \frac{\alpha}{2},
$$

from which $L^{\prime}$ is known as a function of $\alpha, U$ and $I$.

The same method permits varying the angle $\alpha$ successively, and repeating the operation, commencing each time with the same voltage at the terminals $U$, and thus tracing the entire characteristic of an alternator operating upon a dead resistance.

The above method gives immediately the values of the direct and transverse armature reactions. As to the coefficient of self-induction of the stray field $\omega s$, it may be determined for any given alternator by the method indicated later on.

A test may then be made of the two alternators coupled together, without any angular difference of phase between them. The e.m.f.'s. $E_{1}$ and $E_{2}$ are then in simple opposition of phase, and the difference $E_{1}-E_{2}$ will produce a resultant current which may be regulated in strength by regulating the difference of excitation, and which current is dephased by nearly $90^{\circ}$. The diagram is given in Fig. $2 \mathrm{I}$, where $O C_{1}$ and $O C_{2}$

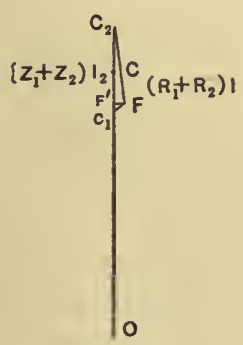

FIG. 2 I.

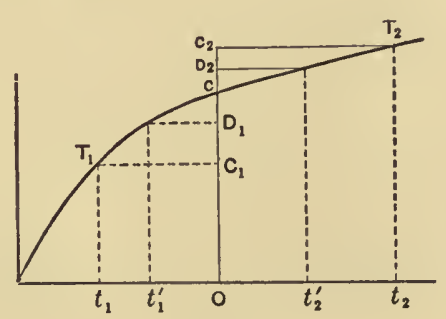

FIG. 22.

are the two internal e.m.f.'s. The difference $C_{1} C_{2}$ represents the fall of potential due to the impedance of the circuit of the armatures, and which can be decomposed into two rectangular straight lines, $C_{1} F$ representing the total ohmic drop $\left(R_{1}+R_{2}\right) I$ due to the current, and $C_{2} F$ the total reactive drop in the armatures. Projecting $F$ upon $C_{1} C_{2}$, a vector $C_{2} F^{\prime}$ is obtained, which differs but little from $C_{2} C_{1}$, and which represents the fall of potential of the two machines due to direct reaction. If the characteristic of total excitation be then drawn as in Fig. 22, this drop will represent the sum of the two drops due to the reactive current, of which one $C C_{1}$ is positive and the other $C C_{2}$ 
is negative. It is easy to mark these off on the characteristic. Inversely, the drops due to the reactive current about the point $C$ may be deduced, and thus the coefficient $K$ of direct reaction. It is sufficient, starting from the point $C$, to trace two segments, $C C_{1}, C C_{2}$, representing the two drops, and to trace the horizontal $C_{1} T_{1}, C_{2} T_{2}$; thence the abscissas $\mathrm{Ot}_{1}, \mathrm{Ot}_{2}$, which represent the virtually lost ampere-turns. If the segments $C D_{1}, C D_{2}$ are known, which represent the e.mf.'s. of dispersion, and if the horizontal straight lines are drawn through $D_{1}$ and $D_{2}$, the corresponding abscissas $t_{1}{ }^{\prime}$ and $t_{2}{ }^{\prime}$ permit of calculating exactly the back ampere-turns, $t_{1} t_{1}{ }^{\prime}, t_{2} t_{2}{ }^{\prime}$, represented by the armature, and which should have equal magnitudes.

Method No. 2. Applicable to a Single Synchronous Machine Operating upon an Actual Conducting System. When only one alternator is available for the test, it is not possible to proceed so conveniently as in the last case, and, in particular, the plan of testing with variable angles of coupling must be given up.

A similar test to that which we have indicated above can, however, be made by driving the alternator on open circuit as a synchronous motor supplied from the conducting system on which it is to be employed (supposing the factory to have other alternators already installed) or by a current furnished from some other alternator of equivalent power. The alternator, or alternators, serving as the source, will then be excited in such a manner as always to maintain the voltage constant at the terminals of the alternator under test which operates as a synchronous motor; this voltage being the

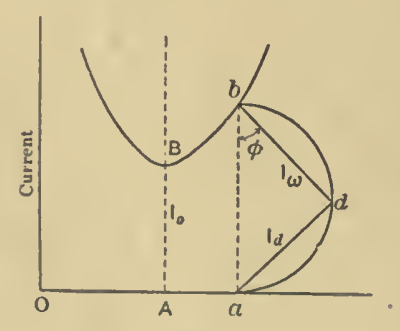

FIG. 23. normal voltage of operation, the excitation of the motor is to be varied, as if it were desired to obtain the "V-curve" of constant voltage. The latter gives by its minimum ordinate $A B$ (Fig. 23) the value of the ohmic losses (at current $I_{0}$ ), and the indication of the condition of excitation $O A$ corresponding to a power-factor equal to unity $(\cos \phi=I)$ -at least on the hypothesis that the effects of harmonics in the e.m.f. are inconsiderable. For any other excitation $\mathrm{Oa}$, the strength of the reactive current may be obtained by constructing upon $a b$ a triangle of which the angle at $b$ is given by the wattmeter. 'The side $b d=I_{u}$, differs little from $B A$, that is to say, from the active current on 
open circuit; ad then represents the reactive current $I_{d}$. The value of the reactive current delivered or received by the motor may then be deduced from the $\mathrm{V}$-curve for all values of excitation. If reference is made to the characteristic in Fig. 24, on which $O C$ represents the normal e.m.f. at the terminals, the knowledge of $A a$ gives for each value of the current $I_{d}$ the corresponding value of the total lost ampereturns $O F=A a$. A curve of these ampere-turns may then be drawn as a function of $I_{d}$, and the total fall of potential $C D$ thus deduced from the chart for every value of the armature current. This method does not separate the reaction into two parts according to theory, but it gives exactly the required result which makes it possible to calculate the fall of potential for each value of the current and of the phase with respect to the mean e.m.f. $C D$ which the armature designed to supply.

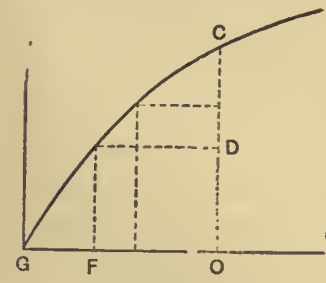

Fig. 24.

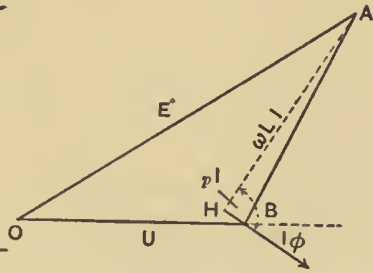

FIG. 25.

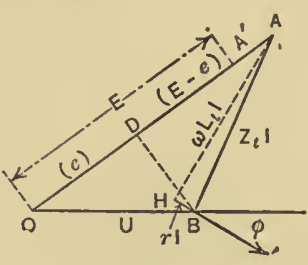

FIG. $25 a$.

To complete this indication it is sufficient to know the transverse reaction. This may be determined readly enough by the same experiment if care be taken to measure the phase angle, $\phi$, of the current with respect to $U$, the mean e.m.f. at the terminals. Let us, then, draw (Fig. 25) the synchronous motor diagram with $O A$ to represent the internal induced e.m.f., $E$, and $O B$ the mean voltage, $U$, at the terminals; then the geometrical difference, $A B$, will represent the fall of potential due to impedance, which may be resolved into two vectors, one of which $(B H)$ represents the ohmic drop, and the other $(H A)$ the fall of potential $w L^{\prime} I$ calculated as a function of the transverse reaction. Knowledge of the angle $\phi$ and the current strength $I$ enables the point $H$, and also the direction-line $H A$, to be determined. Knowing, from the experiments set forth below, the angle of lag, $\phi$, of the internal e.m.f., $E$, (whose direction, $O A$, can thus be drawn) it is only necessary to take the point of intersection of $O A$ with the direction-line $H A$ to locate the point $A$, and, consequently, to be able to complete the triangle $O H A$. The length of 
$H A$ gives, at once, the value of the transverse inductance $L^{\prime}$. The same determination can therefore be made over again with increasing values of $\phi$, by simply increasing the excitation each time. The greater the lag-angle, $\phi$, the more accurately $L^{\prime}$ will be determined.

It may be noted, moreover, (Fig. $25^{a}$ ), that if a line $(B D)$ is drawn from $B$ perpendicular to $O A$, the portion intercepted $(O D)$ corresponds to the e.m.f. $e$ obtained with no-load. The difference $D A^{\prime}=E-e$ therefore gives, in volts, the value of the armaturereaction produced by the reactive component of the output current.

When the angle at $A$ (Fig. $25^{a}$ ) is very small, we have substantially,

$$
D A=Z_{t} I \cos A=\omega L_{t} I_{d} \text {. }
$$

Let

$$
Z_{l}=\sqrt{\gamma^{2}+\left(\omega \circ L_{t}\right)^{2}} \text {. }
$$

If we let $L_{d}=$ the direct reaction, then, for a very small variation of the excitation we can write

whence

$$
D A^{\prime}=\omega L_{d} I_{d}
$$

$$
A A^{\prime}=\omega\left(L_{t}-L_{d}\right) I_{d} .
$$

Instead of evaluating in the preceding test the active current $I_{v v}$ in order to deduce the reactive current $I_{d}$, it would be easy, if a measuring motor were at hand, to make this deliver directly to the shaft of the alternator operating as a synchronous motor the necessary power for driving the motor, in such a manner that $A B$ on the $\mathrm{V}$-curve (Fig. 23) becomes nil. But the same result may be obtained yet more easily when a steam alternator unit has to be tested, by admitting to the engine just enough steam to satisfy the losses both of the engine and alternator, so that the alternator only receives a reactive current. With this object, the steam admission may be regulated in such a manner that the alternator, excited so as to give on open circuit the normal e.m.f. $U$ of the system, runs idly in synchronism; then no change is made either in steam admission or in the pressure, and the operations are conducted entirely on the electric side of the alternator, connecting this with the system and varying its excitation so as to develop the $\mathrm{V}$-curve. It is possible to measure in advance the electric power necessary to drive the alternator and its steam engine on open circuit, and thus to deduce the total losses on open circuit. The power wasted may thus be measured by the steam-engine indicator-diagram, which permits of determining the constant of the curve of steam consumption as a function of the power produced 
(a curve which generally is nearly a simple straight line); it is also possible to separate the mechanical losses, first making this test without field-excitation, and then exciting the field-magnets so as to obtain the normal e.m.f. ${ }^{1}$

Summing up, this test permits of determining, with a fair approximation, the losses on open circuit and then on load, without being obliged to actually develop these losses by full-load in the alternator as well as the corresponding heatings.

From an electrical point of view the same test permits of determining the total values of the direct reaction as a function of the reactive current, and the constant $L^{\prime}$ of the transverse reaction. If it is desired to analyze these phenomena more completely, the value of $\omega s$ may be determined as follows:

Determination of $\omega s$. For this purpose the following considerations are made use of (the basis of which is the method of calculation of the short-circuit current given by Kapp, which does not lend itself to experimental verification):

Let $O N M$ be the characteristic of excitation; that is to say, the curve of the armature e.m.f. as a function of the exciting ampereturns. Suppose the excitation to be constant and equal to $O A$ ampere-turns, giving an e.m.f. represented by $A M$. Suppose the reactance $\omega s$ sought for to be known and also the short-circuit current $I_{c c}$. If a point $P$ is taken on the curve whose ordinate is equal to $\omega s I_{c c}$, the corresponding segment of the abscissa $A H$ will measure the back ampereturns of the armature $K N I_{c c}$. If the calculated values of $\omega s$ and of $K$ are taken, the direction of the straight line $A P$ is known, and the line may be traced from which the value of $I_{c c}$ may

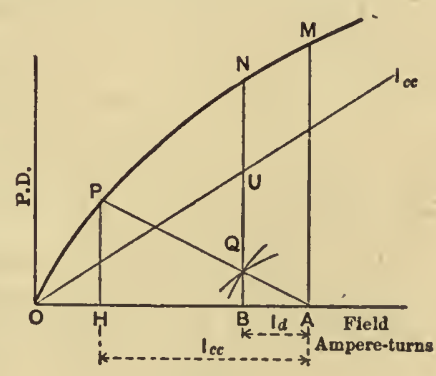

Fig. 26. be deduced. This is the construction of Kapp. We shall take up, on the contrary, the inverse problem, supposing $I_{c c}$ determined by experiment and seeking to deduce from it the two constants $K$ and $\omega s$.

The following observation may then be made. If the segment $A H$ be taken as the measure of the short-circuit current $I_{c c}$ and a segment $A B$ as the measure of any other reactive current $I_{d}$ to the

The total efficiency of the engine and alternator unit may also be obtained later at load by means of the engine diagram. 
same scale of volts at the terminals corresponding to this reactive delivery, this segment will be equal to the ordinate $Q N=U$, taken between the curve and the straight line $A P$. In fact, the back ampereturns will then be equal to $A B$, and the loss of voltage by dispersion equal to $B Q$, by reason of similar triangles. Besides, the point $Q$ divides the straight line $A P$ in the ratio of the current $I_{d}$ to the shortcircuit current $I_{c c}$.

Thus arranged, suppose that any reactive current $I_{d}$ be taken experimentally at the corresponding e.m.f. $U$; the point $Q$ will be determined by these two conditions: its vertical distance from the curve of excitation is equal to $U$ and its radial distance from the point $A$ is equal to $\frac{I_{d}}{I_{c c}} \times A P$.

The point is found, therefore, at the intersection of two new curves that are easily drawn: a curve parallel to $O N M$ traced at a vertical distance $U$ below the former, and a curve homothetic to the curve of excitation with respect to the point $A$, with homothetic ratio $\frac{I_{d}}{I_{c c}}$. These two curves are parallel in their rectangular parts, and separate as much one from the other as the point $M$ is selected further beyond the bend in the characteristic. They would coincide if the point $\dot{M}$ were below the bend. The experiment should therefore be made with an excitation $O A$ sufficient clearly to pass the bend.

The test is made by causing the alternator to operate first on short-circuit, and then upon a reactance-coil having an open magnetic circuit, or upon an under-excited synchronous motor, giving $\cos \phi$ less 0.20 , that is to say, a current almost entirely reactive.

It is understood that if not only one, but also several, reactive circuits are tried, the straight line $P Q$ will be still better determined thereby, and consequently ws will be known with correspondingly greater precision.

Analogies between this Method and that of Potier-Behrend. It is possible also to follow a somewhat different course, by causing the alternator under test to operate upon an inductive circuit with $\cos \phi$ nearly o (for example, upon a synchronous motor driven by a motor adjusted in a manner to produce just the power consumed at light load), and by varying the excitation of the alternator; in this manner a constant reactive-current curve is obtained, from which may be deduced, by the method of M. Potier; the coefficient of the 
back ampere-turns, and the coefficient of the stray field, but which is not directly applicable to the ordinary problem of the calculation of the ampere-turns necessary for constant voltage.

This method is one of those which was devised by Mr. B. A. Behrend, who recently published a number of applications of it. It is wholly different from the preceding, in which the voltage is maintained constant at the terminals instead of the current. The same author employs also another method, which consists in dividing the field magnets into two equal parts to which are given exciting currents of opposite sign and slightly different strength, so as to develop in the armature a certain current, which is necessarily reactive while developing a mean flux-density sensibly equal to that in normal operation. This test may appear equivalent to that which is obtained by means of two alternators mechanically coupled and set at opposite phase (see preceding Method No. I), with the simplification, however, that only one alternator is employed; but as the current thus obtained is only a reactive current, the conditions are not identical with those of the preceding method, but only equivalent to method No. 2 above described (see the case of the alternator operating as a synchronous motor on no-load). It is not, besides, sufficiently rigorous except for alternators having a large number of poles, because when there are but few poles the presence of two poles of the same sign side by side upon the field magnet at two points on the latter would seem to modify notably the conditions of the magnetic circuit. Consequently, Method No. I is preferable when it can be employed.

Method No.3. For the Determination of Transverse Reaction (coefficient L). Besides the preceding methods, several others may also be pointed out which are very simple for the determination of the transverse reaction, and which avoid the objection so often made to the diagram in which this reaction appears. Moreover, the oscillograph (or ondograph) permits the real angle of dephasing $\psi$ to

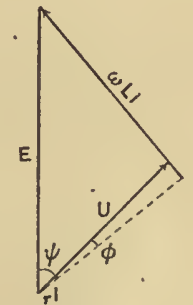

FIG. 27. be measured between the internal e.m.f. $E$ (Fig. 27) and the current, and gives immediately, in consequence, the value of $L$, when the alternator operates upon a non-inductive resistance $\phi=0$, by the equation

$$
\tan \psi=\frac{\omega L I+U \sin \phi}{r I+U \cos \phi}=-\frac{\omega L}{r+\frac{U}{I}},
$$


from which

$$
\omega L=\left(r+\frac{U}{I}\right) \tan \psi
$$

With the oscillograph, for example, it suffices to place upon the shaft, or upon one of the pole pieces of the rotating field-magnet (Fig. 60), a contact segment $S$ upon which rub two brushes $B B^{\prime}$, connected in series with a battery, one of the oscillographs $O$ of a double-oscillograph apparatus, and the electromagnet $e$ which controls the release of the shutter. The contact segment $S$, insulated from the base, closes the circuit upon its passage below the brushes, and opens it again at the precise moment when the brush $B$ leaves it. A segment is selected large enough in order that the shutter shall have time to open before the rupture of the circuit, in order that the latter may then be photographed upon the plate in the form of a vertical line intersecting the straight-line zero furnished by one of the oscillographs.

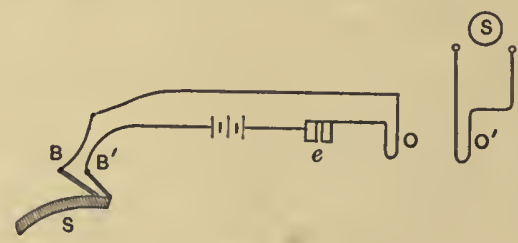

Fig. 28 .

The second oscillograph of the system, $O^{\prime}$, is connected to the terminals of the alternator and serves to register the e.m.f. $U$ at the terminals. Two experiments are made on two different plates, or upon the same plate after having slightly displaced the zero-line produced by the oscillograph $O$, so as to distinguish the two different records. The e.m.f. at open circuit is then marked upon it, and next the e.m.f. when the circuit is closed upon a dead resistance, at the same time that the current $I$ delivered thereto is measured. In these two tests the distance is measured from the zero of the curve of e.m.f. to the point of intersection by the vertical with the zero-line. The difference between these two lengths thus measured determines the displacement of phase of the e.m.f. at the terminals, that is to say, the angle of dephasing sought (taking for the value of $2 \pi$ the length of a period measured upon the plate, and taking the ratio of the measured retardation to this length). It is sufficient to take the values of $\psi$, of $I$, and of $U$ in the preceding formula in order to determine the transverse reaction $L$.

Figs. $13 a, 1_{4} a,{ }_{5} a$ and 16 (Chapter I) represent an example of the 
determination of $L$ made by this method in the laboratory of the Société Sautter-Harlé \& Company of Paris, which employs with success the methods described in this note. The curves of the first three figures have been obtained by means of the author's oscillograph at the terminals of an alternator of $350 \mathrm{~K} . \mathrm{W}$., and represent the periodic curves of the difference of potential between these terminals when the alternator works on open circuit, then when delivering 62 amperes, and finally 102 amperes, its normal load. The effective value of the e.m.f. was II55 volts in star. The vertical lines represent the tracings produced by the contact of the contact-maker $S$. Figs. $1_{3} a$ and $1_{5} a$ are printed upon one and the same sheet of paper, by causing the vertical lines to coincide, from which Fig. I6 is produced, which shows clearly in evidence the dephasing between the internal e.m.f. $E$, and the pressure at terminals $U$. The dephasing reaches $24^{\circ} 30^{\prime}$ in the test with 102 amperes, and $19^{\circ}$ in the test with 62 amperes. In applying the preceding formula (with $r=2.5 \mathrm{ohms}$ ) the values are obtained:

$$
\begin{aligned}
& \omega L=(2.5 \times 62+\text { II } 55) \times 0.344=7.26 \mathrm{ohms}, \\
& \omega L=\left(2.5 \times \mathrm{IO}_{2}+\mathrm{II}_{55}\right) \times 0.566=7.8 \mathrm{I} \mathrm{ohms,}
\end{aligned}
$$

which differ little (and would perhaps be equal if greater precision had been taken in the measurement of the phase-difference between the two curves). In practice one would take the mean value $7.5 \mathrm{ohms}$.

It is evident that it is easy thus to determine the constant of distortion, and this justifies the employment of the theory of the two reactions rather than the rough method of the "curve of short-circuit," which combines together the two often very different reactions.

The ondograph gives the same result, if one marks off successively upon the same sheet of paper the e.m.f. upon open circuit and then that on closed circuit, and each time the mark obtained when the apparatus is traversed by the current coming from the brushes $B B^{\prime}$. It is possible to dispense with making this mark, if the ondograph is. driven, not by a synchronous motor, but by a flexible coupling connected mechanically to the shaft of the alternator (with the interposition of gear wheels, as in the recording mechanism of Francke).

Moreover, even in the absence of the preceding analyzing apparatus, one can approximately obtain the transverse reaction, or rather its ratio to the direct reaction, with unsaturated field-magnets, by sending, as M. Herdt has already suggested, into the alternator arma- 
ture at rest, alternating currents derived from any available external source, and measuring the apparent self-induction by the method of Joubert for the two characteristic positions of the field-magnet poles. The simplest method applicable to a star-wound alternator consists of sending an alternating current $I$ through one of the phases which divides between the two other phases, starting at the neutral point, and which is received at its emergence by a connection applied to the two terminals $A_{2} A_{3}$. Fig. 29 represents the connections: $a$ is an auxiliary alternator of which the excitation is adjusted at will in order to vary the current $I$. It is easy to see that an alternator thus traversed by a current is placed in the same conditions as if it were fed by three-phase currents at the moment when one of these currents passed through its maximum. This connection being made, the field-magnet is arranged so that the armature poles have their axes directed in

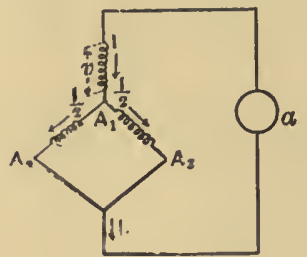

FIG. 29. a line with those of the field-magnet poles, and then in such a manner that these axes are directed midway between the fieldmagnet poles. In each case the self-induction developed by the current $I$ is measured according to the method of Joubert; the ratio of the two measures is that of the direct self-induction to the transverse selfinduction, or of the coefficients $K$ and $K_{\imath}$ applicable to these two reactions. If the difference of potential $u$ is measured between $A$ and the center of the star $O$, the ratio $\frac{u}{I}$ represents the impedance of one phase. From this the inductance may be determined, since the resistance of one phase is known. This measure made in the second position indicated above, gives, therefore, the precise and approximate value of the transverse inductance sought. It must be remembered that this inductance also comprises the inductance of dispersion; therefore has the value

$$
\omega L=\omega \lambda+\omega s,
$$

calling $\omega \lambda$ the transverse reaction properly so called, not including the dispersion $\omega s$.

Repeating the experiment for various values of the current, the constancy of the coefficient $L$ may be determined. 


\section{APPENDIX A}

IF Eq. $(A)$ on page $\mathbf{1} 6$ be solved completely for $i$, the solution will include a transient exponential term depending upon the point of the E.M.F. cycle at which the circuit is closed. It is a well-known fact that this transient term becomes negligible a short time after the circuit is closed, and that when the impressed E.M.F. is sinusoidal the current $i$ will settle down to a sinusoidal form.

In the present case both of the active E.M.F.'s $\left(e_{1}\right.$ and $\left.e_{2}\right)$ are assumed sinusoidal and of the same frequency; their resultant is therefore sinusoidal as will be the current produced thereby. Then all the variables involved are sinusoidal and may be represented by vectors in such a manner as to indicate clearly their phase-relations.

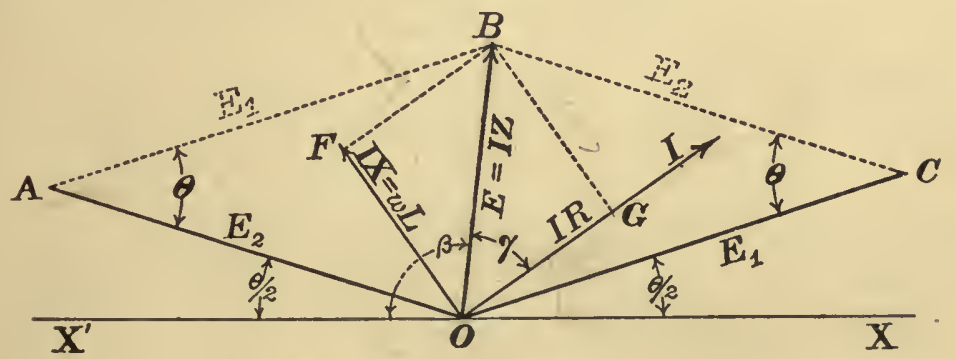

FIG. A

Referring to Fig. $A$, draw $\overline{O C}$, to designate by its length and direction the phase and magnitude of the internal or induced E.M.F. $E_{1}$ of the generator, and $\overline{O A}$ to designate in a similar manner the internal or induced E.M.F. $E_{2}$ of the motor. Then the resultant or vector-sum of $E_{1}$ and $E_{2}$ will be $E$, designated by the line $\overline{O B}$.

The horizontal line $O X$ is taken arbitrarily as the zero of phase, ${ }^{1}$

${ }^{1}$ In Fig. $A$ the instantaneous values of the various variables (e.g., $e_{1}$ and $e_{2}$ ) are given by the vertical projections of the corresponding vectors (multiplied by $\sqrt{2}$, as the latter revolve counter-clockwise at constant angular velocity, $\omega=2 \pi n$, where $n=$ cycles per second. The diagram is shown at the instant $t=0$. 
and the $\angle C O X=\angle A O X=\frac{\theta}{2}$. This corresponds to the equations for $e_{1}$ and $e_{2}$ on page 16 , which show that when $t=0, e_{1}$ has a phase $+\frac{\theta}{2}$ and $e_{2}$ falls short of exact opposition $\left(180^{\circ}\right)$ by the same angle $\frac{0}{2}$, where $\theta$ is the angle between $E_{1}$ and $E_{2}$ projected backwards. $E_{2}$ may be said to lag behind $E_{1}$ by $180^{\circ}+\theta$, or to lead $E_{1}$ by $180^{\circ}-\theta$.

The current produced in the circuit of the two armatures will be, $I=E / Z$ and will lag behind $E$ by the angle $\gamma$ whose tangent is $X \div R$; where $R, X$ and $Z,\left(=\sqrt{R^{2}+X^{2}}\right)$, are respectively, the resistance, the reactance, and the impedance of the whole circuit, including the impedance of the connecting line and the synchronous impedance of the two armatures; and $X=2 \pi n L=\omega L$, where $L$ is the inductance of the whole circuit.

By trigonometry

and since

$$
B=\sqrt{E_{1}^{2}+E_{2}^{2}-2 E_{1} E_{2} \cos \theta}
$$

$$
E=I Z, \text { and } I=\frac{E}{Z}
$$

we have

$$
I=\frac{E}{Z}=\frac{\sqrt{E_{1}^{2}+E_{2}^{2}-2 E_{1} E_{2} \cos \theta}}{Z}
$$

and the current-phase (referred to $O X$ ) is $\pi-\beta-\gamma$.

These two relations stated algebraically, give as the instantaneous value of $i$,

$$
i=\frac{\sqrt{2} \sqrt{E_{1}^{2}+E_{2}^{2}-2 E_{1} E_{2} \cos \theta}}{Z} \sin (\omega t+\pi-\beta-\gamma),
$$

which is the same as the value of $i$ given at the top of page $\mathbf{1} 7$.

The other forms of equations for $i$ and $I$ given on page 17 are deducible from the diagram of Fig. $A$. 


\section{APPENDIX B}

\section{Data Concerning Synchronous Motors of American MANUfacture}

The Tables A and B, given herein below, contain data characteristic of good American practice in the manufacture of synchronous motors. In comparing these data with those characteristic of good European practice, as given in Part I, on page 5, due allowance should be made for the rating conditions in the two cases. Thus, the normal frequency is 60 for the American motors, and 40 for the European motors; and the kilovolt-amperes per horsepower are 746 in America and 736 in Europe. The power-factor assumed as a basis for rating is equal to unity for American motors. It is presumably the same, though not stated, for European motors.

TABLE A.-SINGLE-PHASE AND THREE-PHASE SYNCHRONOUS MOTORS OF AMERICAN MANUFACTURE

\begin{tabular}{|c|c|c|c|c|c|c|c|c|c|c|c|c|}
\hline \multirow[b]{2}{*}{ 产 } & \multirow[b]{2}{*}{$\begin{array}{l}\frac{0}{0} \\
\frac{0}{0} \\
\dot{0} \\
\dot{0} \\
\dot{z}\end{array}$} & \multirow[b]{2}{*}{ 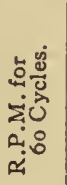 } & \multicolumn{2}{|c|}{ Pulley. } & \multirow[b]{2}{*}{ 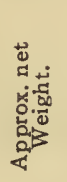 } & \multicolumn{4}{|c|}{ Single-phase. } & \multicolumn{3}{|c|}{ Three-phase. } \\
\hline & & & 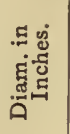 & 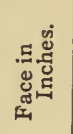 & & 它 & $\begin{array}{l}\dot{\lambda} \\
\dot{\leftrightarrow} \\
\dot{\leftrightarrow}\end{array}$ & 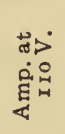 & 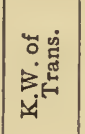 & 空 & 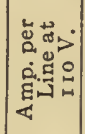 & 岁㤐 \\
\hline $\mathrm{AT}^{*}$ & 4 & 1800 & 8 & $3 \cdot 5$ & 715 & 6.5 & 79 & 56 & 6.5 & Io & $45 \cdot 6$ & 10 \\
\hline $\mathrm{AT}^{*}$ & 4 & 1800 & I0 & $4 \cdot 5$ & 1070 & 13. & 82.5 & 108 & I 2 & 20 & 89.2 & $17 \cdot 5$ \\
\hline $\mathrm{AT}^{*}$ & 4 & 1800 & 12 & $5 \cdot 5$ & I 535 & 22.5 & 83 & 185 & 20 & 35 & 155 & 30 \\
\hline ATB† & 6 & 1200 & 15 & 8 & 2250 & $32 \cdot 5$ & 85 & 265 & 30 & 50 & 220 & 45 \\
\hline ATB $\ddagger$ & 6 & 1200 & 16 & II & $25^{80}$ & $43 \cdot 5$ & $87 \cdot 5$ & 335 & $37 \cdot 5$ & 67 & 287 & 55 \\
\hline ATB $\ddagger$ & 6 & 1200 & I6 & 14 & 3120 & 65 & 89 & 495 & 55 & 100 & 422 & 80 \\
\hline $\mathrm{ATB} \ddagger$ & 8 & 900 & $2 \mathbf{I}$ & 17 & 4380 & 87 & $89 \cdot 5$ & 660 & 75 & I34 & $5^{6} 5$ & IIo \\
\hline $\mathrm{ATB}_{+}^{+}$ & 10 & 720 & 26.5 & 23 & 6630 & I30 & 90 & 980 & IIO & 200 & 840 & 160 \\
\hline
\end{tabular}

* Self-excited.

† Separately excited.

$\ddagger$ Has direct-connected exciter. 
TABLE B.-SINGLE-PHASE AND THREE-PHASE SYNCHRONOUS MOTORS OF AMERICAN MANUFACTURE

\begin{tabular}{|c|c|c|c|c|c|c|c|c|c|c|c|c|}
\hline \multirow[b]{2}{*}{$\stackrel{\aleph}{\Leftrightarrow}$} & \multirow[b]{2}{*}{$\begin{array}{l}\dot{8} \\
\frac{0}{0} \\
\dot{0} \\
\ddot{\circ} \\
\dot{0} \\
\dot{z}\end{array}$} & \multicolumn{2}{|c|}{ Pulley. } & \multirow{2}{*}{ 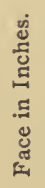 } & \multirow{2}{*}{ 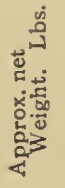 } & \multicolumn{4}{|c|}{ Single-phase. } & \multicolumn{3}{|c|}{ Three-phase. } \\
\hline & & 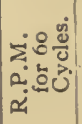 & 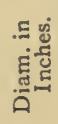 & & & $\begin{array}{l}P^{\circ} \\
=\end{array}$ & 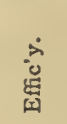 & 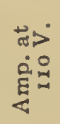 & 岁哭 & $\stackrel{0}{\circ}$ & 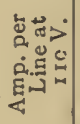 & 离 \\
\hline G- I & 6 & | 1200 & I I & 9 & $\mathrm{I} 49^{\circ}$ & 23 & 85 & I 82 & & 36 & I 58 & $89 \cdot 3$ \\
\hline $\mathrm{G}-3$ & 6 & I 200 & I 5 & 12 & I 940 & $4 \mathrm{I}$ & $S_{7.2}$ & 318 & & $6 I$ & 263 & $9 \mathrm{I}$ \\
\hline $\mathrm{G}-5$ & 8 & 900 & 20 & 12 & 3150 & $5^{8} \cdot 5$ & $87 \cdot 3$ & 445 & & $9 \mathrm{I} .5$ & 394 & 91 \\
\hline $\mathrm{G}-7$ & 8 & 900 & 20 & I5 & 4150 & 82.5 & 88.0 & 636 & & 123 & $5^{26}$ & 9I.t \\
\hline G- 9 & 8 & 900 & 20 & $2 \mathrm{I}$ & 6500 & 120 & $89 \cdot 5$ & 9 ro & & 186 & 787 & 92.5 \\
\hline G-II & I 2 & 600 & 30 & 22 & 8000 & I $2 \mathrm{I}$ & 90.0 & 9ro & & I86 & 787 & 93.0 \\
\hline $\mathrm{G}-\mathrm{I} 3$ & I 2 & 600 & 30 & $2 S$ & 9600 & I 70 & 90.5 & 1272 & & 250 & 1050 & 93.7 \\
\hline
\end{tabular}

All these machines have rotating poles and open slot stationary armatures. The field-poles are equipped, in addition to the standard field-coils, with a short-circuited winding similar to an inductionmotor, which is utilized to make the machine self-starting as an induction-motor, and also as a "damper" to prevent "hunting" when running in synchronism. When starting, polyphase machines will develop from 20 to 50 per cent of their normal torque with from roo to 250 per cent of the normal input in kilovolt-amperes. The single-phase machines are not self-starting. 


\section{APPENDIX C}

\section{USE OF SYNCHRONOUS MOTORS FOR IMPROVING POWER-FACTOR IN AMERICA}

IT is well known that the power-factor of a system supplying current to rotary converters at various substations can be materially improved by over-exciting the field of the rotary converters. In some cases this benefit was considered of enough importance to warrant the use of rotary converters which are shunt-wound only and consequently can produce no compounding effect.

In a motor-generator set composed of a synchronous motor driving a continuous current generator the compensating effect due the over-excitation of the synchronous motor-element is retained without sacrificing the compounding effect of the combination. This is one reason why motor-generator sets have been preferred in many cases to rotary converters.

Owing to the fact that the compensating effect is the same as that produced by a condenser the synchronous motor producing the "condenser effect" is often called a "synchronous condenser" or a "rotary condenser," and also a " synchronous compensator." The Standards Committee of the American Institute of Electrical Engineers gives the preference to another term, "phase-modifier," as appears from the following definition given in the "Standardization Rules" :

"A Synchronous Phase-modifier, sometimes called a Synchronous Condenser, is a synchronous motor, running either idle or under load, whose field-excitation may be varied so as to modify the powerfactor of the circuit, or through such modification to influence the voltage of the circuit." 
The practice of designing the synchronous motor portion of motor-generator sets with special reference to their use as "synchronous condensers" has become quite general in America. Many articles and papers have appeared on the subject. A partial list of the authors is given at the end of the book.

The following item, taken from The Electrical World, refers to an interesting case where synchronous condensers have been used to great advantage:

(From the Electrical World, March 9, 1912, p. 548.)

Overrating of Motor-generator Sets to Improve Power-factor. All motor-generator sets installed by the Detroit Edison Company are now specified with their motor-elements sufficiently larger than the generators to insure that, fully loaded, the motors can be operated at a leading power-factor of 80 per cent to improve the power-factor of the system. Operators are instructed to increase the field-excitation of their machines until the motors take full load current at the leading power-factor. As the result of this practice, power-factors approaching within 97 per cent of unity have been observed at the bus of the generating plant.

The Detroit Edison Company, through its Engineering Department, has kindly placed at the disposal of the Translator the following detailed information regarding this case:

\section{(From the Engineering Department of the Detroit Edison Co.)}

The motor-generator sets referred to in the above item are synchronous machines installed in the substations. They are supplied with 4600 volt A. C., and deliver 600 volt D. C. for railway, and 250 volt D.C. for the lighting, service. All are over-excited more or less; and the station operators are instructed to adjust the field so that the motors always take the full load current at a leading power-factor. This power-factor varies from 50 to 95 per cent.

The total induction-motor load on the systems is about $19,000 \mathrm{KW}$. with a power-factor varying from 60 to 85 per cent, lagging. The lagging current of such a load would lower the power-factor at the power-house bus considerably, but by means of the over-excited machines in the substations a leading current is provided to counteract the lagging one, and a very good power-factor is thus maintained. 
The extent to which the power-factor at the power-house bus is affected by the over-excitation of the synchronous motors is best shown by a vector diagram.

This was figured from simultaneous readings on the turbogenerators at the power-house, and the synchronous motors at the substations. The average power-factor of the turbo-generators with a load of $3 \mathrm{I}, \mathrm{I} 00 \mathrm{KW}$., under normal running conditions, is about 95 per cent, lagging. The reactive component of this is $975^{\circ} \mathrm{K} . \mathrm{V} . \mathrm{A}$., lagging. The total load taken by the synchronous motors is 10,735

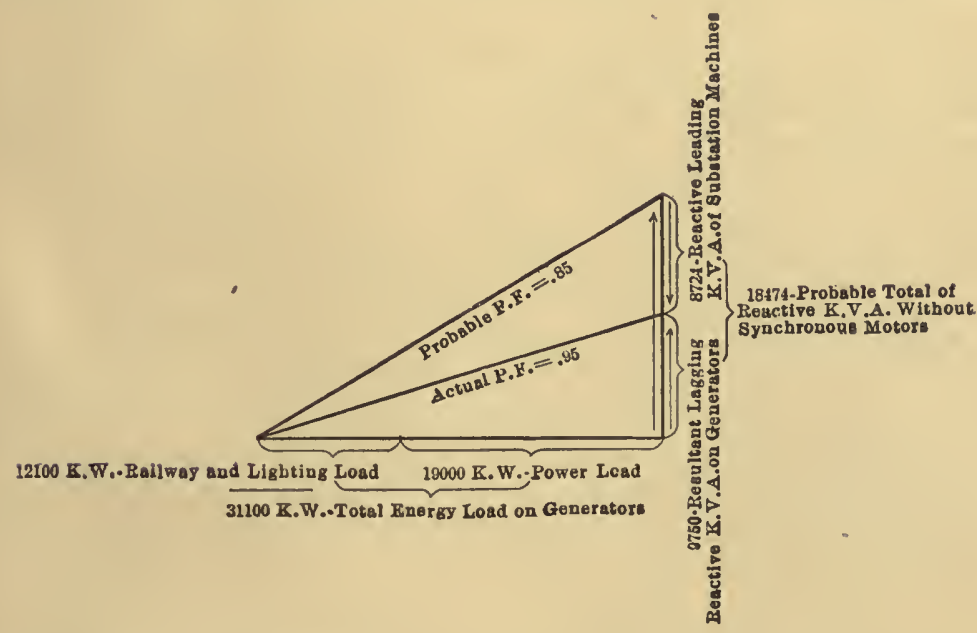

$\mathrm{KW}$. with a power-factor varying from 50 to 95 per cent leading, and the summation of all its reactive components at the various power-factors is $8724 \mathrm{~K}$.W.A. This leading component counteracts an equal lagging one, leaving a resultant reactive lagging component of 9750 K.V.A. on the whole system, corresponding to a power-factor of 95 per cent. Without this leading component the lagging component would be 18,474 K.V.A., which would mean a power-factor of 85 per cent. Hence it is seen that a Io per cent rise in power-factor is obtained by over-excitation of the synchronous motors in our substations.

Regarding the overrating of the motor-ends of the motorgenerator sets, the following two examples of rating might be mentioned: 
Machine No. I. (Installed under the old system.).

Motor: Class, 10-530-720.

Volts, 4400.

Amperes, 73 per phase.

Exciter: Class, 6-7-720.

Volts, 125 .

Amperes, 56 .

Generator: $500 \mathrm{KW}$. direct-connected.

Maciinse No. 2. (Installed under the new system.)

Motor: Class, 10-700-720.

Volts,' 4400.

Amperes, 92.

Power-factor, 0.80 .

Exciter: Class, 6-8-720.

Volts, I25.

Amperes, 64 .

Generator: $500 \mathrm{KW}$. direct-connected.

It will be noticed that the motors of the new machines are larger, and are specified to operate at 80 per cent power-factor. This was necessary to allow for over-excitation in the case of machines of recent design. The old machines, however, will stand considerable over-excitation, because the design of motors was formerly more liberal in overload capacity.

A. A. M. 


\section{INDEX}

Adams, C. A., résumé of paper of, 225 Advantages and disadvantages of synchronous motors, 140

Air-gap, length of, 164

local corrections of, $25 \mathrm{I}$

Algebraical relations deduced from the diagram, 37

Alternating current, starting by, I०6

-field motors, xvi

synchronous motors, 145

Alternators, construction of, and application of calculation of reactances, 267

Mordey, 38, 47, 53

ordinary, polyphase form of, 9

single-phase, case of, 264

testing according to the theory of two reactions, 270

Ampere-turns in the case of unsaturated armature, diagram of, $24 \mathrm{I}$

Armature, case of a saturated, 248 reaction, 92

self-inductance of, 242

reactions, calculation of, 236 reduction to the single-direct reaction, 174

Annual operating cost, saving in, 75

Arnold, E., 236

\section{B}

Bedell and Ryan, ro3

method of determining phaseangle, $\mathbf{3} 39$

Behn-Eschenburg short-circuit curves, I32

Bipolar diagrams, 33

principle of, 32
Blakesley's method of representing operative conditions, 20

Blondel, Prof. André, 236

C

Calculation of constants, $25^{2}$ of reactions, 252

Case of symmetrical polyphase motors, I9

Cassel, power transmission, $5^{2}$

Circuit, or line, and compensation, 65

Classification of single-phase and polyphase motors, xvi

Comparison between synchronous and induction-motors, 90 of outputs, 62

Compensation, economics of, 72 with respect to the generators, 86

to the line or circuit, 65

Complex variables, equation of the synchronous motor by the method of, 23

Compound-excitation, 196

Constant field motors, xvi potential, supply system, I००

V-curves for, 200

Constants, calculations of, $25^{2}$

Construction, $\mathrm{I}-3$

Converter operation, representation of with constant potential at primary terminals and brushes, I 80

rotary, characteristic features of, I 94

lag-characteristics of, $2 \circ 3$

special applications of, 223

Cornu, on oscillations, I 26

Corrected diagram, first application of, 95 
Cost, annual operating, saving in, 75 of equipment, saving in, 72

Coupled synchronous motors, diagrams of, 166

Crocker, Dr. F. B., 207

Current-controllers, 109

limit-circle of, 37,47

-minimum, existence of, 55

of synchronous motors, effect of, on distribution-systems, 64

-supply voltage, value of, I 83 values, 182

Curve of reaction curve, $6 \mathbf{I}$

Curves, characteristic test, 132

indicating power developed, 44

\section{$\mathrm{D}$}

Damper of Hutin and Leblanc, $\mathbf{I} 26$

Damping circuits, 221

of oscillations, I 26

Definition of synchronous motors, xvii

Determining maximum power, 5 I

Determination of reactive current, 95 , I98

Determining the practical stability of synchronous motors, $5^{\text {I }}$

Detroit Edison Co., on power-factors, 288

Diagram, corrected, first application of, 95

fundamental, 177

of ampere-turns in the case of unsaturated armature, $24 \mathrm{I}$

of E. M. F.'s and current of an alternator with unsaturated armature and with saturated field magnet, 239

of the first kind, applications of, 35

of the first kind, use of, 57

of the second kind, 40

transformations, 149

Diagrams, simplified, 104

Direct current, starting by, 106

Distributing system, economy of compensation for, 80
Distribution-systems, effect on of current of synchronous motors, 64

-voltage, regulation of, 85

Dynamometer, Pronybrake method, I34

torsion type, 137

E

Early types of synchronous motors, $x$ viii

Economy of compensation, for the distributing system, 80

Efficiency, measurement of, I34

Electric current supply to rotary converters, 177

Elementary expansion of polyphase synchronous motors, 9

of single-phase synchronous motors, 14

E. M. F. diagrams, 150

fluctuations in rotary converters, 220

regulation of supply by compounding of the generator, 210

Empirical coefficients, 255

Equal electromotive forces, case of, 5

phase, lines of, 36,47

Equation of the synchronous motor by the method of complex variables, 23

Equations of synchronous motors, I5

Equipment, saving in cost of, 72 table, 76

Excitation, different values of, 200 compound, I96

constant, lines of equal power occurring with, 36

of synchronous motors, 26

separate, II 7

application in case of, 206

Experimental properties, 5

tests, 137

Experiments at South Foreland, xvii

Expression for reactive current, 6I 
F

Ferraris, Galileo, I 45

Field due to a commutated current, I 2 I

magnets, stray field of, 242 with divided winding, 252

-saturation, influence of, on stability, IоI

Fort Wayne Company, starting method, I I 4

method of current commutating, 30

Forces, equal electromotive, case of, 5 unequal electromotive, case of, 7

Fresnel's method of vectors, 20

Fundamental diagram, I77

G

Generator, compounding of, to regulate supply of E. M. F., 210 power-factor of, 192

Generators, compensation with respect to, 86

Gramme commutators, II 5

Graphical representation of operative conditions, 20

Guilbert, C. F., 236 .

\section{H}

Herdt, L. A., 236

Hobart and Punga, 236

Hopkinson, Dr. J., I 5

analytical theory, I5

Hutin and Leblanc's damper, I 26

Hutin, on oscillations, I 3 I

I

Induction and synchronous motors, comparison between, 90

Influence of field-saturation on stability, I I I

of wave-form of E. M. F., IO2

Initial synchronizing, theory of, I I 7

Janet, M., 24
K

Kapp type of synchronous motors, 90

L

Labour starting method, II3

synchronous motors, 5 I

Lag of current, variations of reactance with, 92

Leblanc, M. Maurice, I4

on oscillations, I 3 I

starting method, I I3

Length of air-gap, 164

Limit-circle of current, 37

Line of equal power occurring with constant excitation, 36

Lines of equal phase, 36,47

\section{M}

Measurement of efficiency, I34

Mordey alternators, $38,47,53$

\section{$\mathrm{N}$}

Notation, 172

for study of operation, 3 I

Numerical examples, 53, 69, 75, 84, 88

\section{O}

Observations on the E. M. F., induced at the poles, 108

Oerlikon type of motor, I33

Operation of a motor with constant excitation, 49

with constant excitation, I00

Operations, factors determining the practical condition of, 176

Operative conditions, representation of, 20

Oscillations, damping of, 126

of converters connected in parallel, 220

of synchronous motors, 122

long-period, I30

Outputs, comparison of, 62

Overrating of motor-generator sets to improve power-factor, 288 


\section{$\mathbf{P}$}

Parallel working of rotary converters, 2 I9

Perot, M., I03

Phase-angle, determining, I 39

-converters, 224

indicators, 108

Picon, M. R. V., 236

Polyphase alternators, ordinary form of, 9

motors, symmetrical, case of, 19

synchronous motors, elementary expansion of, 9

Power developed, curves indicating, 44 -factor of the generator, 192

improving, 287

output, 50

-values as function of the lagangle, 43

Predetermination of V-curves, 57

Principles of the theory of two reactions, 237

Prony-brake-dynamometer, I34

\section{$\mathbf{R}$}

Reactance coil, use of, III

value of, 183

Reactances, calculation of applied to alternator construction, 267

Reaction synchronous motors, I4I

Reactions, practical calculations of, $25^{2}$

two, principles of the theory of, 237

Reactive counter-ampere-turns of armature $24 \pi$

current, curve of, $6 \mathrm{I}$

determination of, 95,198

expression for, $6 \mathrm{I}$

upper limit of, 202

values for a given voltage

variation as a function of the load, 182

Regulation of distribution-voltage, 85

Revolving field motors, xxi
Rotary converters as generators, 224

as partial generators, 224

conditions of electric current supply to, 177

characteristic features of, I 94

eliminating causes of irregular operation, $2 \mathrm{I} 3$

for transforming direct to alter-

- nating current, 222

lag characteristics of, 203

parallel working of, 2 I9

speed oscillations in, 220

under load, effective characteristics of, 205

Ryan and Bedell, ro3

S

Saving in annual operating cost, 75

in operating cost, table, 77

Self-excitation, 30

-inductance of armature, 242

Separate excitation, I 7

Series-excitation, 27

Short-period oscillations, 122

Shunt-excitation, 28

-winding, possibility of suppressing, 2 II

Single-phase alternators, case of, 264

machines, starting, I 2

synchronous motors, elementary expansion of, 14

Société l'Eclairage Electrique, type of synchronous motors, table, 5

Speed-oscillations in rotary converters, 220

Stability, influence of field-saturation on, ror

of synchronous operation, II motors, determining, $5^{\mathrm{I}}$

variations of, with operating conditions, 52

Starting apparatus, accessory, I08

by alternating current, 106

by direct current, Io6

of single-phase machines, I I 2

Steinmetz notation, 23 
Swinburne's "hedgehog" transformers xix

Synchronism, 107

Synchronous and induction motors, comparison between, 90

operation, stability of, I I

motors with alternating fields, I 45

Symmetrical polyphase motors, case of, I9

\section{$\mathrm{T}$}

Tap voltage, 23I

Table, empirical coefficients, 255,258 , 259

motors of the Société l'Eclairage Electrique, 5

numerical example, 78

saving in cost of equipment, 76

in operating cost, 77

single-phase and three-phase synchronous motors of American manufacture, 285,286

theoretical coefficients, 263

Tesla starting method, II 3

Testing alternators, 270

Theoretical coefficients, 263

comparison with, 26I

form of V-curves, 59

Theory, analytical, of synchronous motors, 15

Thompson, Prof. S. P., 64
Three-part pole, 232

Transverse reaction, method of determining, 279

Two-part pole, 234

\section{U}

Unequal electromotive forces, case of, 7

\section{V}

Value of current-supply voltage, $\mathrm{I}_{3}$ of reactance, 183

Variation of voltage by the split pole, 225

Variations of stability with operating conditions, 52

V-curves, 56

for constant potential, 200

predetermination of, 57

theoretical form of, 59

Voltage regulation by varying the reactance $\mathrm{X}$ in the current, 2 I I

of at terminals, $189, \mathbf{r} 90$

variation by the split pole, 225

\section{W}

"Watted" current, 34

Wave-form of E. M. F., influence of, 102 







$$
\left.5\right|^{A-179}
$$




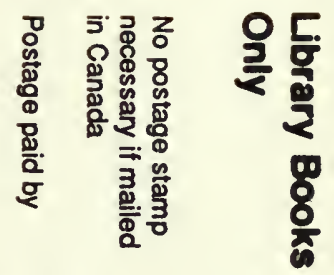

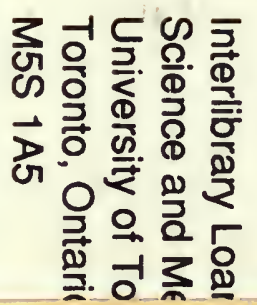

S\&M

A

179 
